\title{
TURBULENT RECTANGULAR JETS
}

\author{
BY \\ MOHAMMED HAMED ALNAHHAL
}

M. Sc., B.Sc. in Mechanical Engineering

A THESIS SUBMITTED IN FULFILLMENT OF THE DEGREE

OF

DOCTOR OF PHILOSOPHY

IN

MECHANICAL ENGINEERING AND AERONAUTICS

AT

\author{
LABORATORY OF APPLIED THERMODYNAMICS \\ DIVISION OF ENERGY, ENVIRONMENT AND AERONAUTICS \\ DEPARTMENT OF MECHANICAL ENGINEERING AND AERONAUTICS \\ UNIVERSITY OF PATRAS \\ RIO 26 504, GREECE
}

Patras, 2010 


\section{CANDIDATE'S DECLARATION}

It is hereby declared that this thesis or any part of it has not been submitted elsewhere for the award of any degree or diploma.

\section{Signature of the Supervisor}

Dr. Thrassos Panidis

Assistant Professor

Laboratory of Applied Thermodynamics

Division of Energy, Environment and Aeronautics

Department of Mechanical Engineering and Aeronautics

University of Patras

Rio 26504

Greece.

Email:panidis@mech.upatras.gr

\section{Signature of the Candidate}

Mr. Mohammed Alnahhal

PhD Candidate

Laboratory of Applied Thermodynamics

Division of Energy, Environment and Aeronautics

Department of Mechanical Engineering and Aeronautics

University of Patras

Rio 26504

Greece.

Email: mhnahhal@mech.upatras.gr mohmd_iut@yahoo.com 


\section{PUBLICATIONS FROM THE PRESENT WORK}

\section{A) Journals}

1. M. Alnahhal and Th. Panidis "Effect of sidewalls on rectangular jets", Experimental Thermal and Fluid Science (33) 5, (2009) 838-851.

2. M. Alnahhal, A. Cavo, A. Romeos, K. Perrakis, Th. Panidis "Experimental investigation of the effect of endplates and sidewalls on the near field development of a smooth contraction rectangular jet". Under review in European Journal of Mechanics- B/Fluids (2011).

\section{B) Conferences}

3. M. Alnahhal, K. Perrakis and Th. Panidis, "Effect of endplates on rectangular jet with and without sidewalls”. ETMM08, 9-11 June 2010, Marseille, France.

4. M. Alnahhal and Th. Panidis, "Effect of endplates on the near field of rectangular jet of different aspect ratio", $4^{\text {th }}$ European Combustion Meeting, 14-17 April 2009, Vienna, Austria.

5. M. Alnahhal and Th. Panidis, "Effect of sidewalls on the near field of rectangular jet", $6^{\text {th }}$ meeting of Fluid mechanics, November 28, 2008, Kozani, Greece. 


\section{Members of Advising Committee}

1. Dr. Thrassos Panidis (Supervisor) Assistant Professor, Department of Mechanical Engineering and Aeronautics University of Patras, Patras, Greece

2. Dr. Ioannis Kallinderis Professor, Department of Mechanical Engineering and Aeronautics University of Patras, Patras, Greece

3. Dr. Kostas Perrakis Lecturer, Department of Mechanical Engineering and Aeronautics University of Patras, Patras, Greece

\section{Members of Examination Committee}

1. Dr. Ioannis Ekaterinaris

Professor, Department of Mechanical Engineering and Aeronautics University of Patras, Patras, Greece

2. Dr. Apostolos Goulas

Professor, Department of Mechanical Engineering Aristotle University of Thessaloniki, Thessaloniki, Greece

3. Dr. Ioannis Kallinderis

Professor, Department of Mechanical Engineering and Aeronautics University of Patras, Patras, Greece

4. Dr. Panayiotis Koutmos

Associate Professor, Department of Mechanical Engineering and Aeronautics University of Patras, Patras, Greece

5. Dr. Dionisios Margaris

Associate Professor, Department of Mechanical Engineering and Aeronautics University of Patras, Patras, Greece

6. Dr. Thrassos Panidis

Assistant Professor, Department of Mechanical Engineering and Aeronautics University of Patras, Patras, Greece

7. Dr. Kostas Perrakis

Lecturer, Department of Mechanical Engineering and Aeronautics University of Patras, Patras, Greece 
To Allah

To my parents, my sisters and brother To my wife, and my parents in law 



\section{CONTENTS}

Acknowledgment

Abstract

Abstract-Greek

List of symbols

iv

List of figures

List of tables

\section{Introduction}

1.1 Background

1.2 Contribution of present thesis

1.3 Open issues and further work

1.4 Thesis outline

\section{Theory}

2.1 Turbulent flows

2.2 Free shear flows

2.3 Boundary layer flows 10

2.4 Turbulent jets 11

2.5 Turbulent jets issuing from rectangular nozzles 13

2.5.1 Description of the velocity field 14

$\begin{array}{ll}\text { 2.5.1.1 Mean velocity fields } & 14\end{array}$

a) Mean streamwise velocity 14

b) Mean lateral velocity 15

c) Mean spanwise velocity 15

d) Spread of rectangular jets 16

e) Decay of centerline mean velocity 17

f) Type of initial boundary layer 18

2.5.1.2 Turbulent velocity fields $\quad 18$

a) Turbulent velocities $\quad 18$

2.5.1.3 Self-similarity concept 19

2.6 Related equations 20

a) Equation of motion 20

b) The integral momentum equation $\quad 23$

c) The integral energy equation $\quad 24$

d) Entrainment hypothesis $\quad 26$

2.7 Initial and boundary conditions of rectangular jets 26

a) Reynolds number $\quad 26$

b) Aspect ratio 27

c) Nozzle type $\quad 27$

d) Sidewalls and/or endplate 28 
4. Experimentation 40

$\begin{array}{lll}4.1 & \text { Experimental Set-up } & 40\end{array}$

4.1.1 Nozzle exit $\quad 40$

4.1.2 Settling chamber $\quad 40$

4.1.3 Blower 42

4.1.4 Endplates and sidewalls $\quad 42$

4.2 Hot wire anemometry 43

4.2.1 Constant temperature hot-wire anemometry (CTA) 43

4.2.1.1 Working principle 44

4.2.1.2 Noise 45

4.2.1.3 Advantages of hot wire anemometry technique 46

4.2.1.4 Disadvantages of hot wire anemometry technique 46

4.2.1.5 Selection of hot wire sensors 46

4.2.1.6 Calibration of hot wire anemometer 47

4.2.1.7 Measuring velocity components 48

4.2.1.8 Specifications of the hot wire anemometry system and experimental conditions of the present investigation $\quad 49$

4.2.1.9 Experimental uncertainty $\quad 50$

4.3 Experimental procedure $\quad 50$

5. Effect of endplate and/or sidewalls on rectangular jets 52

$\begin{array}{lll}5.1 \text { Results and discussion } & 52\end{array}$

5.1.1 Mean velocity field $\quad 52$

5.1.1.1 Longitudinal mean velocity 52

a) Transverse distributions of longitudinal mean
velocities

b) Similarity of longitudinal mean velocity profiles $\quad 57$

c) Spanwise distributions of longitudinal mean velocities 60

5.1.1.2 Lateral mean velocity 62

a) Transverse distributions of lateral mean velocities $\quad 62$

b) Similarity of lateral mean velocity profiles 67

5.1.1.3 Jet's spread rate 68

$\begin{array}{ll}\text { 5.1.1.4 Entrainment and mass flow } & 70\end{array}$

$\begin{array}{ll}\text { 5.1.1.5 Momentum growth in the axial direction } & 71\end{array}$

5.1.1.6 Axial mean velocity decay at various Reynolds numbers 72

5.1.2 Turbulent velocity field 77

5.1.2.1 Longitudinal turbulent velocity 77

a) Transverse distributions of longitudinal turbulent velocities normalized to the centerline velocity $\quad 77$

b) Similarity of longitudinal turbulent velocity profiles $\quad 82$

c) Lateral distributions of longitudinal turbulent velocities normalized to the exit velocity

d) Evolutions of the centerline longitudinal turbulent velocities at various $R e$ 
5.1.2.2 Lateral turbulent velocity

a) Transverse distributions of lateral turbulent velocities normalized to the centerline velocity

b) Similarity of lateral turbulent velocity profiles

95

c) Lateral distributions of lateral turbulent velocities normalized to the exit velocity

d) Evolutions of the centerline lateral turbulent velocities at various $R e$

99

5.1.2.3 Reynolds shear stresses

a) Transverse distributions of Reynolds shear stresses normalized to the centerline velocity

b) Transverse distributions of Reynolds shear stresses normalized to $u^{\prime} v^{\prime}$

5.1.2.4 Triple velocity products

a) Transverse distributions of triple velocity product $\left\langle u^{3}\right\rangle$

b) Transverse distributions of triple velocity product $\left\langle v^{3}\right\rangle$

c) Transverse distributions of triple velocity product $\left\langle u^{2} v\right\rangle$

d) Transverse distributions of triple velocity product $\left\langle u v^{2}\right\rangle$

5.1.2.5 Skewness factor

a) Transverse distributions of skewness factor of the longitudinal velocity

b) Evolutions of the centerline skewness factor of the longitudinal velocity at various $R e$

c) Transverse distributions of skewness factor of the lateral velocity

d) Evolutions of the centerline skewness factor of the lateral velocity at various $R e$

5.2 Overview and physical interpretation of the experimental results 


\section{ACKNOWLEDGMENT}

I would like to thank almighty Allah for everything. Almighty Allah provided me the strength, patience and kind people who helped me to complete my thesis. Those people are:

First and foremost, I am deeply grateful to my supervisor Prof Thrassos Panidis, Department of Mechanical Engineering and Aeronautics, University of Patras for the invaluable assistance, guidance and encouragement that he provided me during the preparation of this thesis and during my stay in Greece. His depth of insight, understanding, involvement and dedicated supervision at every critical stage made possible the completion of this study. Many thanks to the departed Professor D. Papailiou for his help, encouragement and introducing me to Professor T. Panidis. Also, I would like to express my appreciation to the members of the supervision and examination committees.

I would like to thank Prof Kostas Perrakis for his help to me during my stay at LAT. Many thanks to Dr. Alexandros Romeos for the continuous cooperation, help and encouragement. Also, thanks to Dr. Thanasis Giannadakis, my roommate for the motivation and help and Dr. Alexander Cavo and Dr. Alexandros Vouros for their help during my $\mathrm{PhD}$ thesis. Mr. Skouras Giannes for introducing me to hot wire anemometry technique, Mr. Andreas Vouros, Mr. Zafiris Sotiris and Mrs. Papadopoulou Sofia, for their cooperation during my stay here. I would like also to thank Dr. Kalogirou Ioannis. Assistant Professor, Technological Educational Institute of Patras for the assistance that he provided me. Many thanks to everyone who stood beside me during this journey.

I would like to thank the State Scholarship Foundation (I.K.Y) in Greece for the financial support that provided me the ability to complete this research.

I would like also to thank my father "Hamed", my mother "Amina", my father in law "Said", my mother in law "Asma", my sisters "Ruba and Reem", my brother "Amjad", and all of my relatives for their wish and encouragement; and for their everlasting support. I want to thank my wife "Raghda"; she successfully compromised between home and her $\mathrm{PhD}$ work, thank you for your unlimited love, patience and support, good luck in your $\mathrm{PhD}$ thesis. Also, thanks to all of my friends for their encouragement.

Finally, my appreciation is highly directed to Greece and Greek people for their hospitality and generosity. 


\begin{abstract}
Turbulent jet flows issuing from rectangular nozzles are used in many technological and practical applications. Understanding their development and mechanics is important to the design and performance improvement of these applications. Therefore, rectangular jets have been studied extensively over the past decades.
\end{abstract}

Previous investigations have extensively studied the effects of various initial and boundary conditions such as nozzle type, nozzle aspect ratio, nozzle exit turbulence intensity, jet exit Reynolds number. Nozzle type (e.g. smooth contraction nozzle, channel, radially countred nozzle) has been found to affect the development of these jets. While varying Reynolds number affects the mixing of the jet with its ambient either in the near field or in the far field. Nozzle aspect ratio is also an important boundary condition. It has been experimentally shown that aspect ratio influences the dimensionality of flow field, and hence entrainment between the jet and its ambient. Some of these investigations have used sidewalls (two parallel plates attached to the nozzle short sides) to improve the two-dimensionality of the flow fields, and/or endplate (a wall flush at the nozzle exit) to prevent mixing upstream of the nozzle. Despite this fact very few investigators have specifically considered the effect of endplate or sidewalls on jet development.

In the present investigation, the effects of endplates and/or sidewalls on turbulent jets issuing from a rectangular of aspect ratio, $A R=15$ have been studied. The mean streamwise and lateral velocity and turbulent characteristics of four different rectangular jets, namely,

1. jet with no endplate and no sidewalls, NENS,

2. jet with endplate and no endplate, WENS,

3. jet without endplate and with sidewalls, NEWS and

4. jet with endplate and with sidewalls, WEWS,

have been measured, with $x$-sensor hot wire anemometry, up to an axial distance of 35 $D$ under identical inlet conditions. Centreline measurements for the four 
configurations have been collected for three Reynolds number, $\operatorname{Re}_{D}=10,000,20,000$ and 30,000. For $\operatorname{Re}_{D}=20,000$ measurements in the transverse direction were collected at 13 different downstream locations in the range of $x=(0,1,2,2.5,3,4,5,10,15,20$, 25, 30 and 35) nozzle widths.

The jet with no endplate and no sidewalls (NENS) and the jet with endplate and no sidewalls (WENS) produce nearly similar mean and turbulent velocity profiles indicating insignificant effect of the endplates on their development in the absence of the sidewalls.

At $\operatorname{Re}=20,000$, larger mean streamwise velocity values were observed at the edges of the jets (NEWS or WEWS) at distances from nozzle in the range of $x / D=3-30$ whereas the presence of the endplate has an insignificant effect.

The presence or absence of sidewalls is also key factor determining the distributions of the lateral mean velocity component. The presence of sidewalls is associated with lower outward velocities within the edges of the jets and higher inward ones outside the edges. The absence of sidewalls makes the presence of the endplate insignificant and the lateral velocity attains outside the edges low negative values of almost the same level in both cases (NENS, WENS) The presence of an endplate has again some significance only when the sidewalls are present alleviating their effect.

Absence of the endplate in jet with sidewalls, leads to lowest spread rate compared to the case with endplates (comparing jet with no endplate and with sidewalls, NEWS and jet with endplate and with sidewalls, WEWS). The presence of an endplate in the absence of sidewalls has again insignificant effect on the jet's spread rate (comparing jet with no endplate and no sidewalls, NENS and jet with endplate and no sidewalls, WENS).

The effects of endplates and/or sidewalls on the decay rates have been investigated for $R e_{D}=10,000,20,000$ and 30,000. The presence of the endplate in jets with no sidewalls has insignificant effect on decay rate as was observed for all Reynolds numbers. The presence of the endplate in jet with sidewalls, leads to higher decay rates for all Re tested compared to all cases. The decay rates of the jets with no sidewalls in the presence and absence of the endplate (NENS, WENS) seem to reach their asymptotic values at around $R e_{D}=20,000$. But the jets with sidewalls have not reached an asymptotic behaviour even at the highest $R e$ tested. 
The implementation of sidewalls has been found to lead to a decrease of the streamwise turbulent velocity, $u^{\prime}$, at the exit shear layer. Current results indicate that the presence of an endplate has an insignificant effect and the estimates for the root mean square of the streamwise velocity fluctuation $u^{\prime}$, at the exit shear layer can be grouped according to the absence or presence of sidewalls $(3.8 \%$ of the mean centreline velocity for NENS and WENS, and 3.1\% for NEWS and WEWS).

For all turbulent terms, the presence of the endplate has an effect in the presence of sidewalls and the nondimensional streamwise turbulent velocity attains always higher values in its presence (comparing WEWS with NEWS). The profiles of the streamwise turbulent velocity, $\mathrm{u}^{\prime}$ profiles for the jet with no endplate and no sidewalls, (NENS) and the jet with endplate and no sidewalls, (WENS) are almost indistinguishable in the range $x / D=15-35$ but the profiles of the jet with no endplate and with sidewalls, (NESWS) and jet with endplate and with sidewalls, (WEWS) indicate a monotonic increase of the values in the central area of the jets. This indicates that the observed trends are mainly due to the presence of the sidewalls and presence or absence endplate has insignificant effect. 


\section{ПЕРІАНЧН}

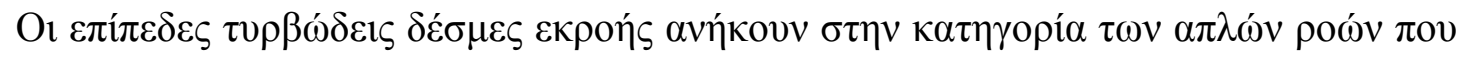

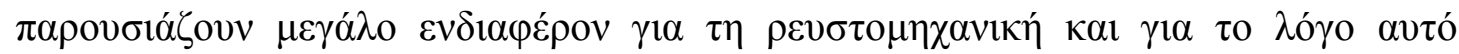

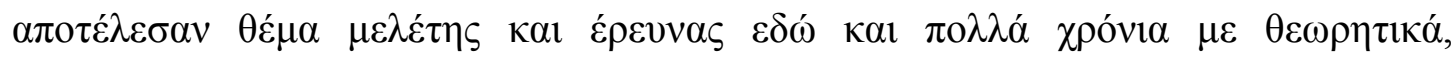

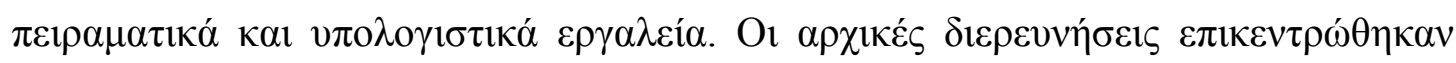

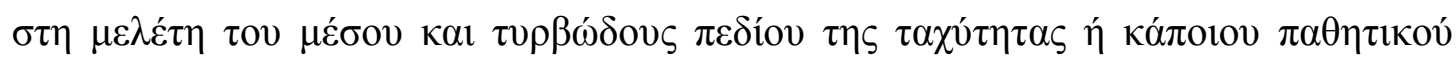

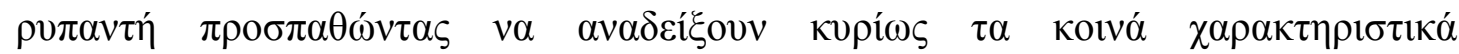

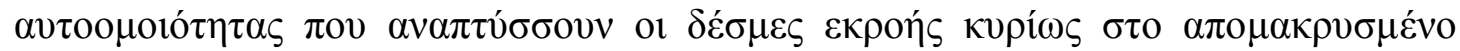

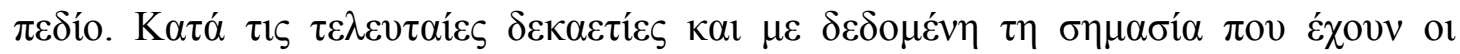

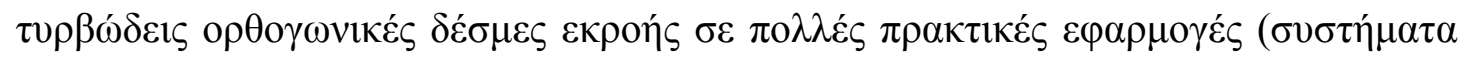

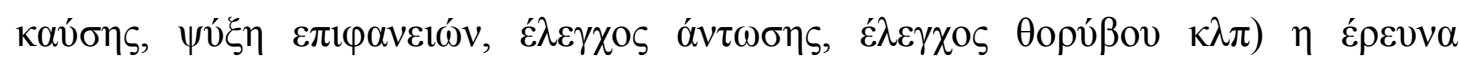

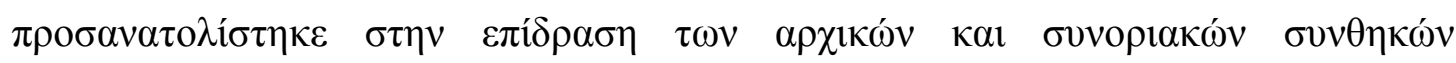

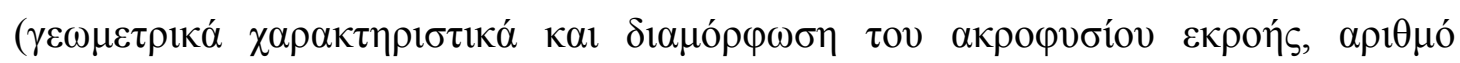

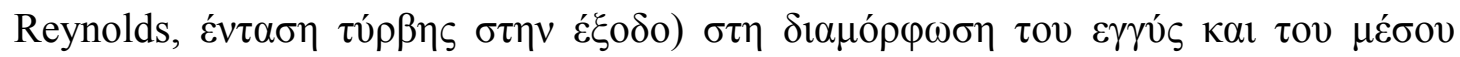
$\pi \varepsilon \delta$ íov.

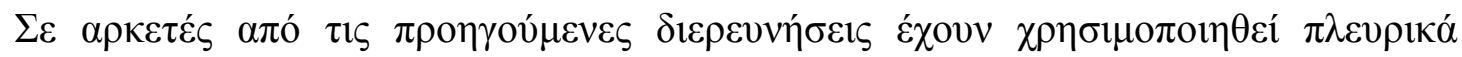

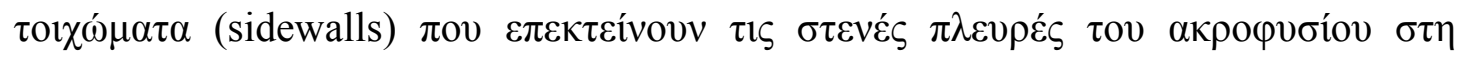

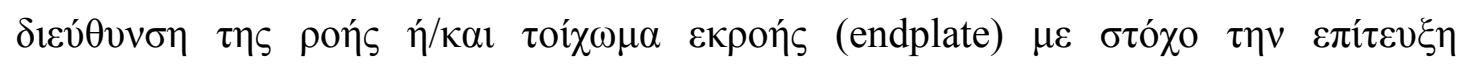

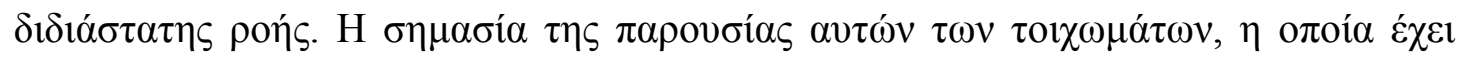

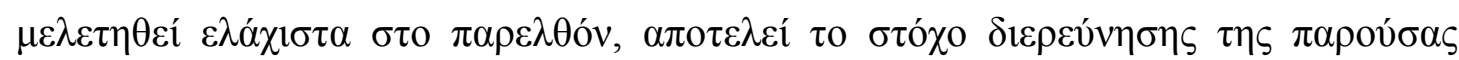

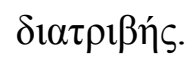

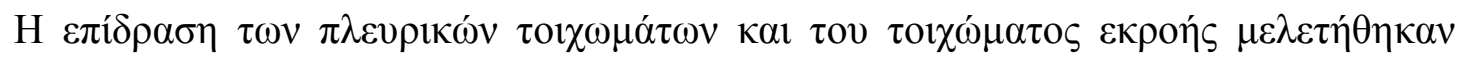

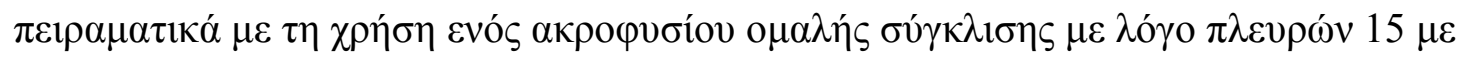

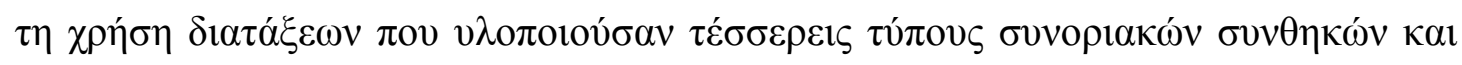

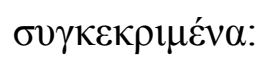

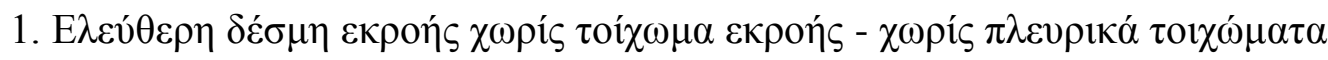
(No endplate - no sidewalls, NENS),

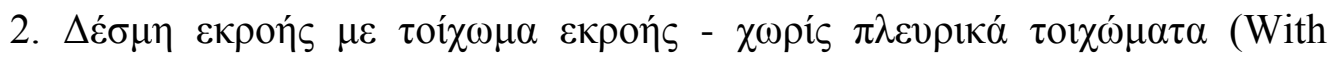
endplate - no sidewalls, WENS),

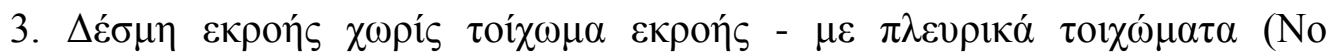
endplate - with sidewalls, NEWS), 


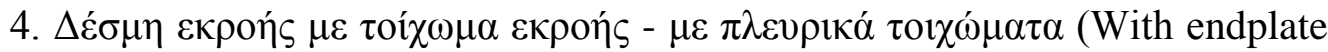
- with sidewalls, WEWS).

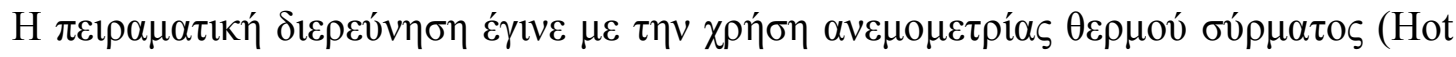

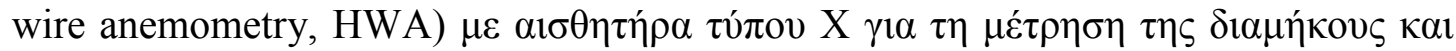

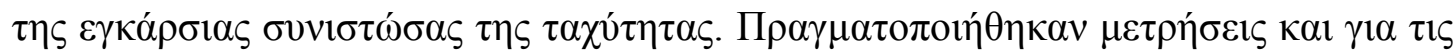

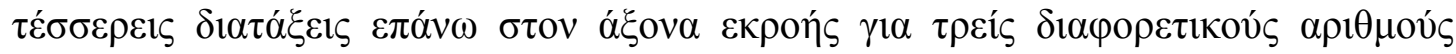

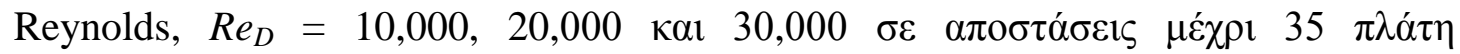

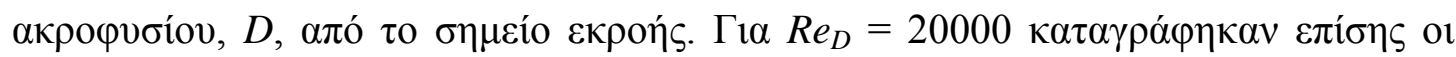

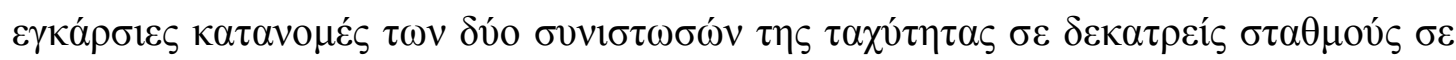

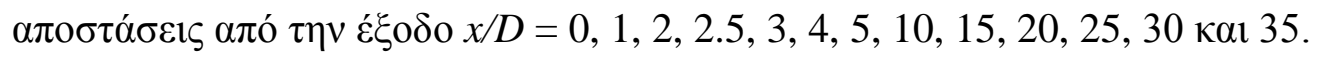

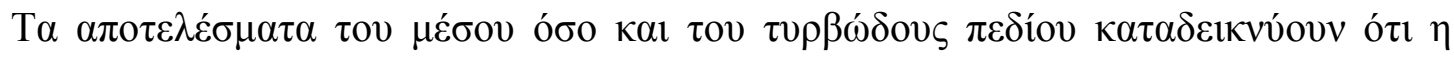

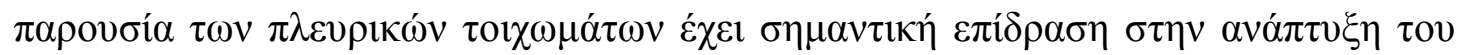

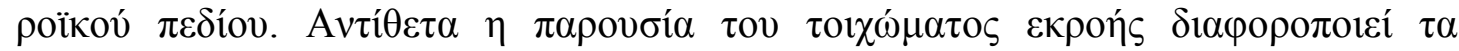

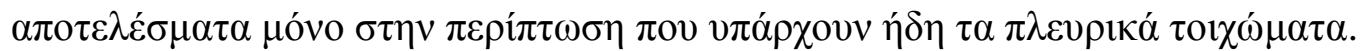




\section{LIST OF SYMBOLS}

$A_{l} \quad$ Spread rate constant

$A_{2} \quad$ Spread virtual origin constant

$A R \quad$ Nozzle aspect ratio, $A R=L / D$

$B_{1} \quad$ Decay rate constant

$B_{2} \quad$ Kinematic virtual origin constant

$D \quad$ Width of the rectangular nozzle, $\mathrm{mm}$

$D_{r} \quad$ Diameter of the round nozzle, $\mathrm{mm}$

$E \quad$ Kinetic energy, $J$

$H \quad$ Shape factor, $H=\delta_{1} / \theta_{m}$

Ir Iridium

$L \quad$ Long side length of the nozzle, $\mathrm{mm}$

$L_{s w} \quad$ Length of the sidewalls, $\mathrm{mm}$

$m \quad$ Mass flow rate (per jet span length), $\mathrm{kg} \mathrm{m}^{-1} \mathrm{~s}^{-1}$

$m_{0} \quad$ Mass flow rate at nozzle exit for NENS jet (per span length), $\mathrm{kg} \mathrm{m}^{-1} \mathrm{~s}^{-1}$

$M \quad$ Momentum flow (per jet span length), $\mathrm{kg} \mathrm{s}^{-2}$

$M_{0} \quad$ Momentum flow at nozzle exit for NENS jet (per span length), $\mathrm{kg} \mathrm{s}^{-2}$

Re Reynolds number, $R e=U_{0} D / v$

$r \quad$ radial coordinates

$S_{u} \quad$ Skewness of the axial velocity

$S_{v} \quad$ Skewness of the lateral velocity

$t \quad$ Time, $\mathrm{s}$

$U \quad$ Mean streamwise velocity, $\mathrm{m} / \mathrm{s}$

$U_{0} \quad$ Mean exit centreline streamwise velocity, $\mathrm{m} / \mathrm{s}$

$U_{c} \quad$ Mean streamwise velocity of the centreline, $\mathrm{m} / \mathrm{s}$

$U_{\infty} \quad$ Free stream velocity, $\mathrm{m} / \mathrm{s}$

$u \quad$ Average velocity in the axial direction, $u=U+u^{\prime}, \mathrm{m} / \mathrm{s}$

$u^{\prime} \quad$ Root-mean-square (rms) of the fluctuating streamwise velocity $\left(\sqrt{ }<u^{2}>\right.$ ), m/s

$\left\langle u^{2} v\right\rangle \quad$ Turbulent transport of $\langle u v\rangle$ by $u, \mathrm{~m}^{3} / \mathrm{s}^{3}$

$\left\langle u^{3}\right\rangle \quad$ Turbulent transport of $\left\langle u^{2}\right\rangle$ by $u, \mathrm{~m}^{3} / \mathrm{s}^{3}$

$\langle u v\rangle \quad$ Reynolds shear stresses, $\mathrm{m}^{2} / \mathrm{s}^{2}$ 


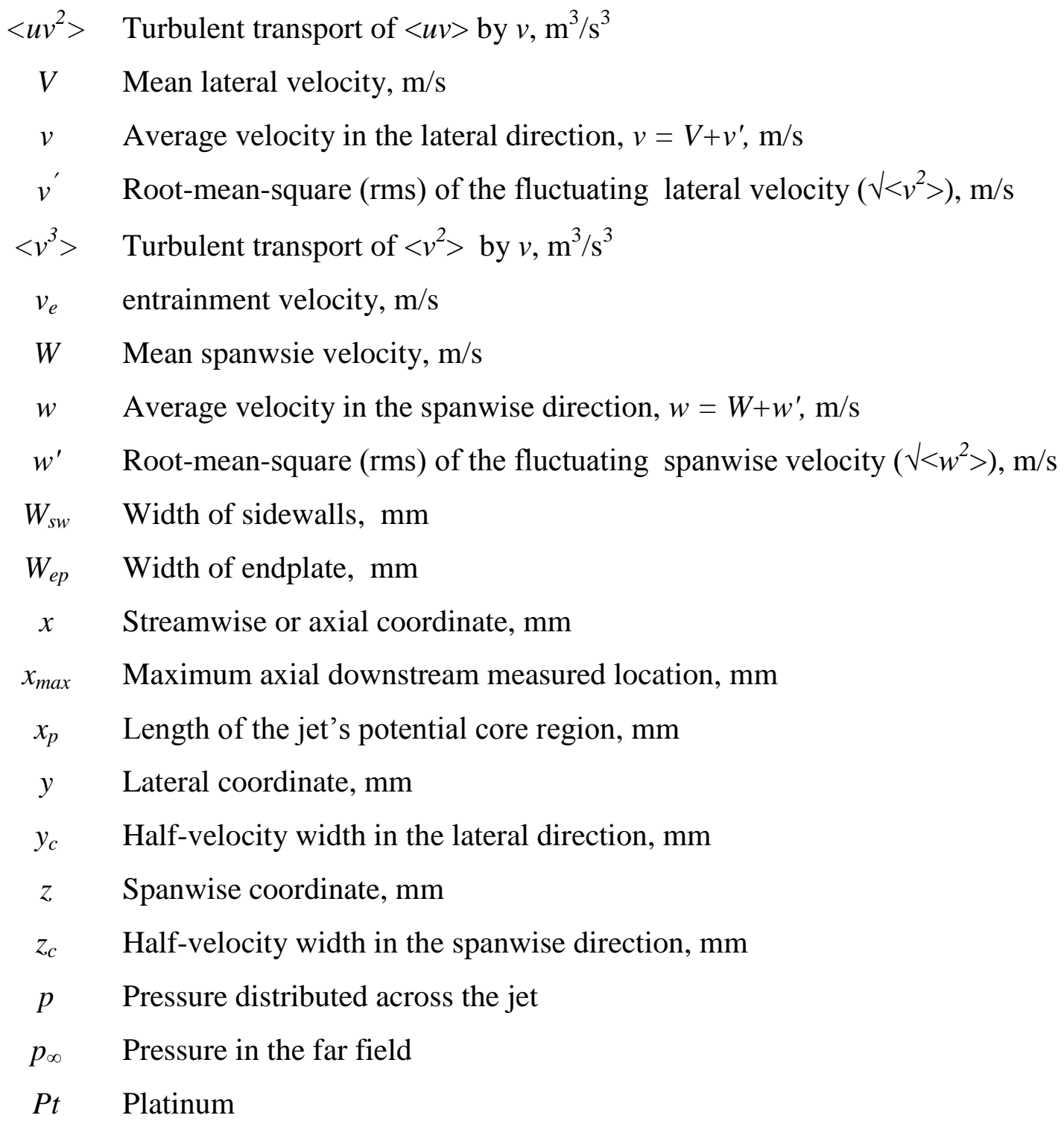

\section{Greek letters}

$v \quad$ Kinematic viscosity of air, $\mathrm{m}^{2} / \mathrm{s}$

$\delta \quad$ Boundary layer thickness, $\mathrm{mm}$

$\delta_{1} \quad$ Displacement thickness, $\mathrm{mm}$

$\tau$ Shear stress, $\mathrm{N} / \mathrm{m}^{2}$

$\theta \quad$ Circumferential coordinate

$\theta_{m} \quad$ Momentum thickness, mm

$\mu \quad$ Dynamic viscosity, N.s $/ \mathrm{m}^{2}$

$\rho \quad$ Mass density of fluid, $\mathrm{kg} / \mathrm{m}^{3}$ 


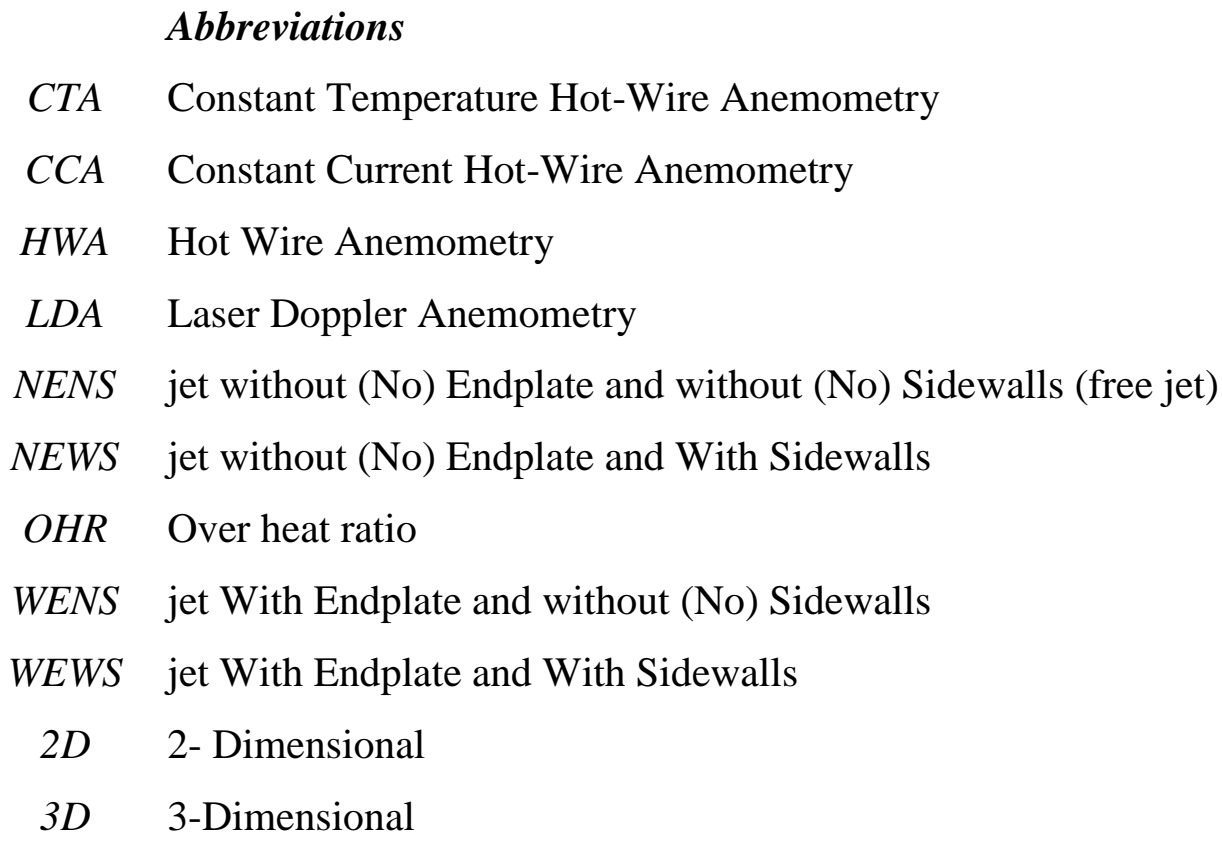

CTA Constant Temperature Hot-Wire Anemometry

CCA Constant Current Hot-Wire Anemometry

HWA Hot Wire Anemometry

LDA Laser Doppler Anemometry

NENS jet without (No) Endplate and without (No) Sidewalls (free jet)

NEWS jet without (No) Endplate and With Sidewalls

OHR Over heat ratio

WENS jet With Endplate and without (No) Sidewalls

WEWS jet With Endplate and With Sidewalls

$2 D \quad 2$ - Dimensional

3D 3-Dimensional 


\section{LIST OF FIGURES}

1.1 Schematic representation of the flow fields of a rectangular jet. Redrawn after modification from Krothapalli et al., (1981)..................... 2

1.2 A schematic view of a rectangular nozzle with sidewalls and endplate..... 4

2.1 Illustrations of two dimensional flows. Redrawn after Pope, (2002)...... 9

2.2 The development of boundary layer for flow over a flat plate and different flow regimes. Redrawn after Çengel, (1997).................. 11

2.3 Illustration of the round jet facility and coordinate. Redrawn after Pope,

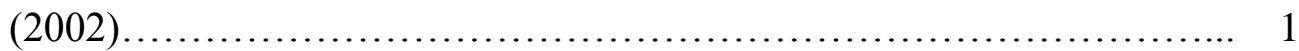

2.4 Orifice shapes used by Mi et al., (2000)........................... 13

2.5 Spanwise profiles of mean streamwise velocity of Quinn, (1992)......... 14

2.6 Development of mean spanwise velocity in the spanwise direction. Data extracted from Krothapalli et al., (1981)........................... 16

2.7 Illustration of jet's development across the minor dimension of a nozzle indicating the location of $y_{c}$. Redrawn after modification from Deo, (2005)

2.8 Definition of mean and fluctuating turbulent of velocity. Redrawn after White, (1994)

2.9 Lateral profiles of the turbulent spanwise velocity at different downstream locations. Data extracted from Cavo et., (2007)..............

2.10 Different types of rectangular nozzles: rectangular channel nozzle, redrawn after Krothapalli et al., (1981), and rectangular nozzle with sharp-edged, redrawn after Deo, (2005).

2.11 Smooth contraction nozzle: a) with sidewalls and endplate, and b) with endplate

3.1 Initial velocity profiles from a sharp-edged orifice plate, a smooth contraction nozzle, and a long pipe for a round jet. Data extracted from Mi et al., (2001).

4.1 Experimental apparatus, a) jet facility, and b) rectangular nozzle with endplate and sidewalls, and c) rectangular nozzle with endplate. (all dimensions in $\mathrm{mm}$ ). 
4.2 Hot wire anemometry components a) typical $\mathrm{x}$-wire probe, and $\mathrm{b}$ ) the Wheatstone bridge. Redrawn after AN-1003, Hot wire \& film anemometry systems, User's Manual................................

4.3 Signal a) before conditioning, and b) after conditioning. Redrawn after AN-1003, Hot wire \& film anemometry systems, User's Manual

4.4 Typical calibration curve of a hot wire anemometer. Redrawn after Goldstein, (1996).

4.5 X-wire configuration. Redrawn after Goldstein, (1996).

5.1 Mean streamwise velocity profiles on the central $x y$ plane.

5.2 Self similarity profiles of mean streamwise velocity on the central $x y$ plane for all test cases.

5.3 Mean streamwise velocity profiles in the central $x z$ plane.

5.4 Mean lateralwise velocity profiles on the central $x y$ plane.

5.5 Self similarity profiles of mean lateralwise velocity on the central $x y$ plane for all test cases.

5.6 Jet half-velocity widths in the stream wise direction.

5.7 Mass flow rate ratio along the jets................................ 71

5.8 Momentum flow ratio along the jets............................ 72

5.9 Axial mean velocity decay at different Reynolds numbers............... 73

5.10 Axial mean velocity decay characteristics at different Reynolds numbers. 75

5.11 Transverse distributions of longitudinal turbulent velocities on the central $x y$ plane normalized to the centreline velocity at different downstream locations

5.12 Self similarity of transverse distributions of longitudinal turbulent velocities on the central $x y$ plane normalized for different configurations: a) NENS, b) WENS, c) NEWS, and d) WEWS

5.13 Turbulent intensity distributions of the streamwise velocity component normalised to the exit velocity.....

5.14 Centreline distributions of longitudinal turbulent velocity for all configuration at Reynolds numbers: a) 10,000, b) 20,000 and c) $30,000 \ldots$

5.15 Reynolds number dependence of the centerline longitudinal turbulent velocity for: a) NENS, b) WENS, c) NEWS and d) WEWS. 
5.16 Turbulent intensity distributions of the lateral velocity component on the central $x y$ plane normalised to the centreline velocity at different downstream locations.

5.17 Self similarity of turbulent intensity distributions of the lateral velocity component on the central $x y$ plane for different test cases: a) NENS, b) WENS, c) NEWS and d) WEWS.

5.18 Turbulent intensity distributions of the lateral velocity component normalised to velocity at the exit.

5.19 Centreline distributions of lateral turbulent velocity for all configuration at various Reynolds numberss: a) 10,000, b) 20,000 and c) 30,000....

5.20 Reynolds number $R e$ dependence of the centerline lateral turbulent velocity for: a) NENS, b) WENS, c) NEWS and d) WEWS

5.21 Reynolds shear stress profiles on the central $x y$ plane normalized to the centreline velocity at different downstream locations

5.22 Self similarity profiles of Reynolds shear stress on the central $x y$ plane for all test cases: a) NENS, b) WENS, c) NEWS and d) WEWS

5.23 Reynolds shear stress profiles on the central $x y$ plane normalized to $\left(u^{\prime} v^{\prime}\right)$ at different downstream locations.

5.24 Profiles of triple velocity product of $\left\langle u^{3}\right\rangle$ on the central $x y$ plane at different downstream locations

5.25 Self similarity profiles of triple velocity product $\left\langle u^{3}\right\rangle$ on the central $x y$ plane for all test cases: a) NENS, b) WENS, c) NEWS and d) WEWS......

5.26 Profiles of triple velocity product of $\left\langle v^{3}\right\rangle$ on the central $x y$ plane at different downstream locations.

5.27 Self similarity profiles of triple velocity product $\left\langle v^{3}\right\rangle$ on the central $x y$ plane for all test cases: a) NENS, b) WENS, c) NEWS and d) WEWS......

5.28 Profiles of triple velocity product of $\left\langle u^{2} v\right\rangle$ on the central $x y$ plane at different downstream locations.

5.29 Self similarity profiles of triple velocity product $\left\langle u^{2} v\right\rangle$ on the central $x y$ plane for all test cases: a) NENS, b) WENS, c) NEWS and d) WEWS......

5.30 Profiles of triple velocity product of $\left\langle u v^{2}\right\rangle$ on the central $x y$ plane at different downstream locations 
5.31 Self similarity profiles of triple velocity product $\left\langle u v^{2}\right\rangle$ on the central $x y$ plane for all test cases: a) NENS, b) WENS, c) NEWS and d) WEWS...

5.32 Skewness profiles of the longitudinal velocity on the central $x y$ plane at different downstream locations.

5.33 Self-similarities of skewness profiles of the longitudinal velocity on the central $x y$ plane for all test cases: a) NENS, b) WENS, c) NEWS and d) WEWS

5.34 Centreline distributions of skewness factor of the longitudinal velocity for all configuration at various Reynolds numbers: a) 10,000, b) 20,000, and c) 30,000 .

5.35 Reynolds number dependence of the centerline skewness factor of the longitudinal velocity for: a) NENS, b) WENS, c) NEWS and d) WEWS...

5.36 Skewness profiles of the lateral velocity on the central $x y$ plane at different downstream locations

5.37 Self-similarities of skewness profiles of the lateral velocity on the central $x y$ plane for all test cases: a) NENS, b) WENS, c) NEWS and d) WEWS..

5.38 Centreline distributions of skewness factor of the lateral velocity for all configuration at Reynolds numbers: a) 10,000, b) 20,000 and c) 30,000 .

5.39 Reynolds number dependence of the centerline skewness factor of the lateral velocity for: a) NENS, b) WENS, c) NEWS and d) WEWS. 


\section{LIST OF TABLES}

4.1 Dimensions of sidewalls and endplates in relation to characteristic dimensions of jet development..

5.1 Jet characteristics of the present jets and previous investigations................................................... 76

5.2 Characteristics parameters of developing jet flows..................... 84 



\section{Chapter 1}

\section{INTRODUCTION}

\subsection{Background}

Turbulent rectangular jets are used in many technical applications in aerospace, mechanical and chemical engineering. They can be found in the propulsion units in both conventional and V/STOL aircrafts, dispersion of pollutant effluents, and gas turbine plants of electric power utilities (Quinn, 1992). In combustion, the mixing between the reactants in diffusion flames, or between the hot reaction products and the fresh reactants needed to sustain combustion, is a crucial part of the combustion process. The mixing occurs in two stages. The initial stage brings relatively large amounts of the reactants together (large-scale stirring). The second stage involves small-scale turbulent mixing, which accelerates the molecular contact between the reactants. Noncircular jets can be used to improve the small and large scale mixing in combustion process (Gutmark \& Grinstein, 1999).

The flow field of a rectangular jet is characterized by the presence of three distinct regions (Figure 1.1), defined by the centreline mean streamwise velocity, $U_{c}$, decay (Krothapalli et al., 1981), namely: (a) the potential core region, with almost constant $U_{c}$, which ends when the two shear layers along the short dimension of the nozzle meet, (b) the two-dimensional region in which the velocity decays at a rate roughly the same as that of a planar jet, and (c) the axisymmetric region, in which the velocity decays as in an axisymmetric jet, the location where the two shear layers along the long dimension of the nozzle meet. A rectangular jet may approximate the behaviour of a plane jet ${ }^{1}$, given that its aspect ratio, $A R=L / d$ (where $L$ is the long side of the nozzle in $z$ direction and $D$ is the short side of the nozzle in the $y$ direction, see Figure 1.1) is large enough, to minimize the importance of entrainment in the spanwise direction, $z$, and hence establish a statistically two-dimensional flow (Pope, 2002).

\footnotetext{
${ }^{1}$ The ideal plane jet is statistically two-dimensional, in the dominant direction of the mean flow, $x$, and the cross-stream coordinate, $y$, whereas statistics are expected to be independent of the spanwise coordinate z (Pope, 2002).
} 


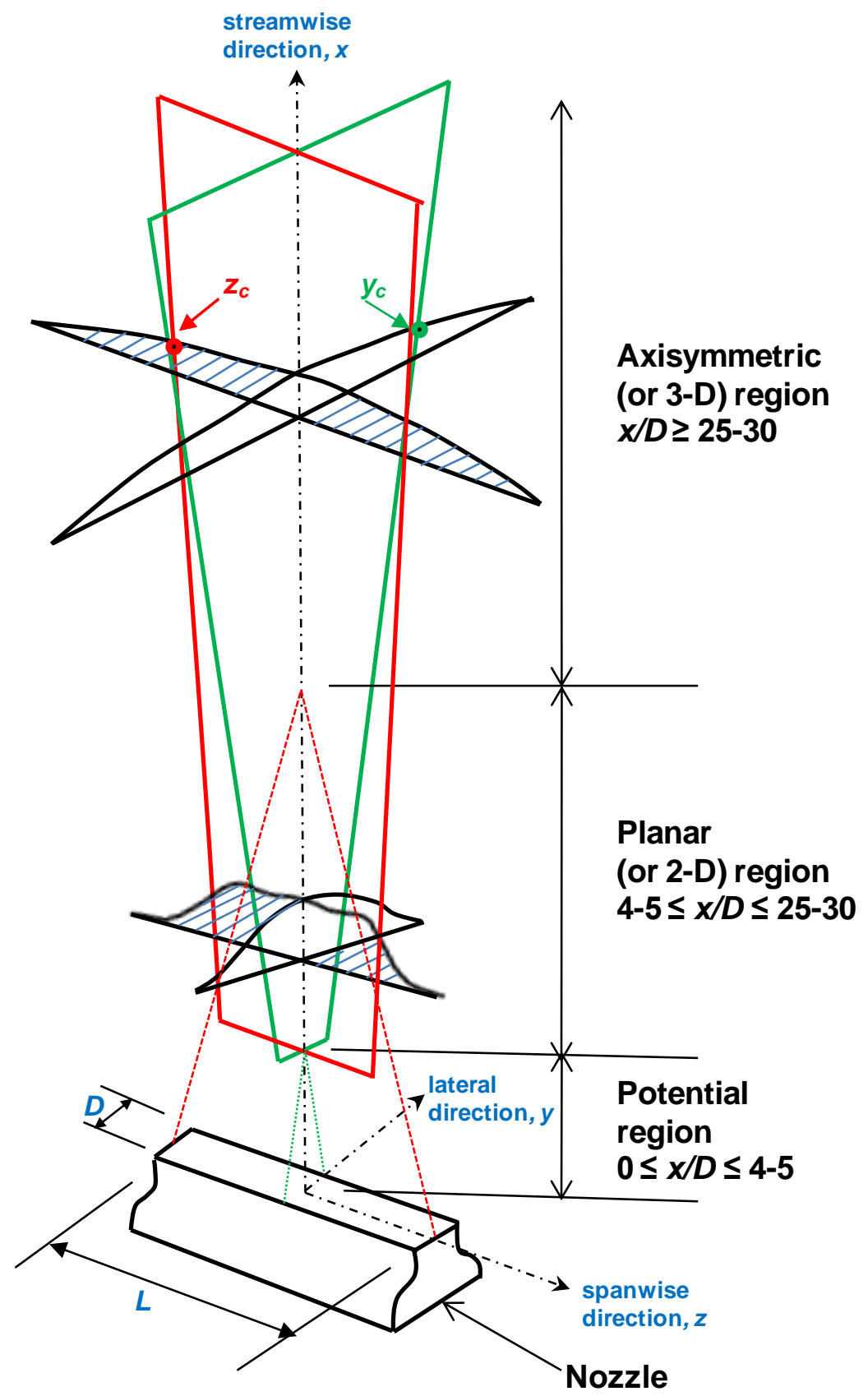

Figure 1.1: Schematic representation of the flow fields of a rectangular jet. Redrawn after modification from Krothapalli et al., (1981).

It is well known that initial and boundary conditions strongly affect the development of turbulent jets issuing from rectangular nozzles. Many experimental investigations have found that the flow fields of rectangular jets are strongly influenced by Reynolds number (e.g. Namer \& Ötügen, 1988, Deo et al., 2008, Suresh et al., 2008). 
Moreover, nozzle's shape (or nozzle type) (e.g. rectangular nozzle, rectangular channel) has been found to affect the evolution of these jets (e.g. Sfier, 1979, Deo et al., 2007b). Also, aspect ratio, which is the ratio of major axis to minor axis of the nozzle, is an important boundary condition, since it also influences the flow fields of rectangular jets (e.g. Quinn, 1992, Mi et al., 2005, Deo et al., 2007a). Krothapalli et al., (1981) have observed that, the point where the jet first assumes axisymmetric behavior goes upstream toward the exit of the nozzle with the decrease of the aspect ratio. In addition, two sidewalls attached to the nozzle's short sides extending in the axial direction (Figure 1.2) have been used in many investigations (e.g. Heskestad, 1965, Gutmarak \& Wygnanski, 1976, Browne et al., 1982, Browne et al., 1984, Deo et al., 2007a) to force the jet to entrain only in the axial and lateral directions and hence ensure the 2-dimensionality of the flow fields. Endplate (i.e. a plate flush at the nozzle exit) has been also used many previous investigations (e.g. Browne et al., 1982, Browne et al., 1984, Quinn, 1992) to prevent entrainment upstream of the nozzle. Figure 1.2 shows the endplate in the presence of the sidewalls.

During the past years, a great number of studies have been devoted to study turbulent jets issuing from rectangular nozzles. A review on the experimental set-ups of these investigations reveals that these set-ups can be classified into four groups based on the presence and/or absence of the sidewalls and/or endplates, namely:

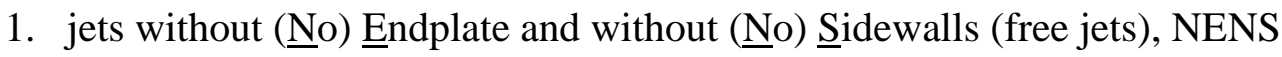
(e.g. Sforza et al., 1966, Trentacoste \& Sforza, 1967, Krothapalli et al., 1981, Namer \& Ötügen, 1988, Papadakis \& Staiano, 1993, Lazanova \& Stankov, 1998, Mi et al., 2005, Cavo et al., 2007, Deo et al., 2007c).

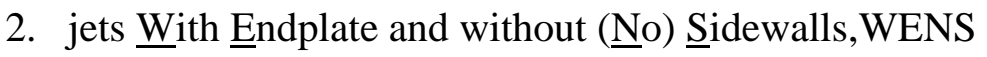

(e.g. Sato, 1959, Sforza et al., 1965, Hussain \& Clark, 1977, Masrsters, 1980, Hitchman et al., 1990, Quinn, 1992, Papadakis \& Staiano, 1993).

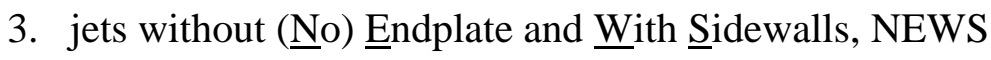

(e.g. Bradbury 1965, Everitt \& Robins, 1978, Deo et al., 2007a, Deo et al., 2007b, Deo et al., 2007c, Deo et al., 2008, Suresh et al., 2008). 


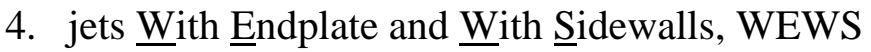

(e.g. Gutmark \& Wygnanski, 1976, Antonia et al., 1983a, Antonia et al., 1983b, Hitchman et al., 1990).

\section{Axial direction, $x$}
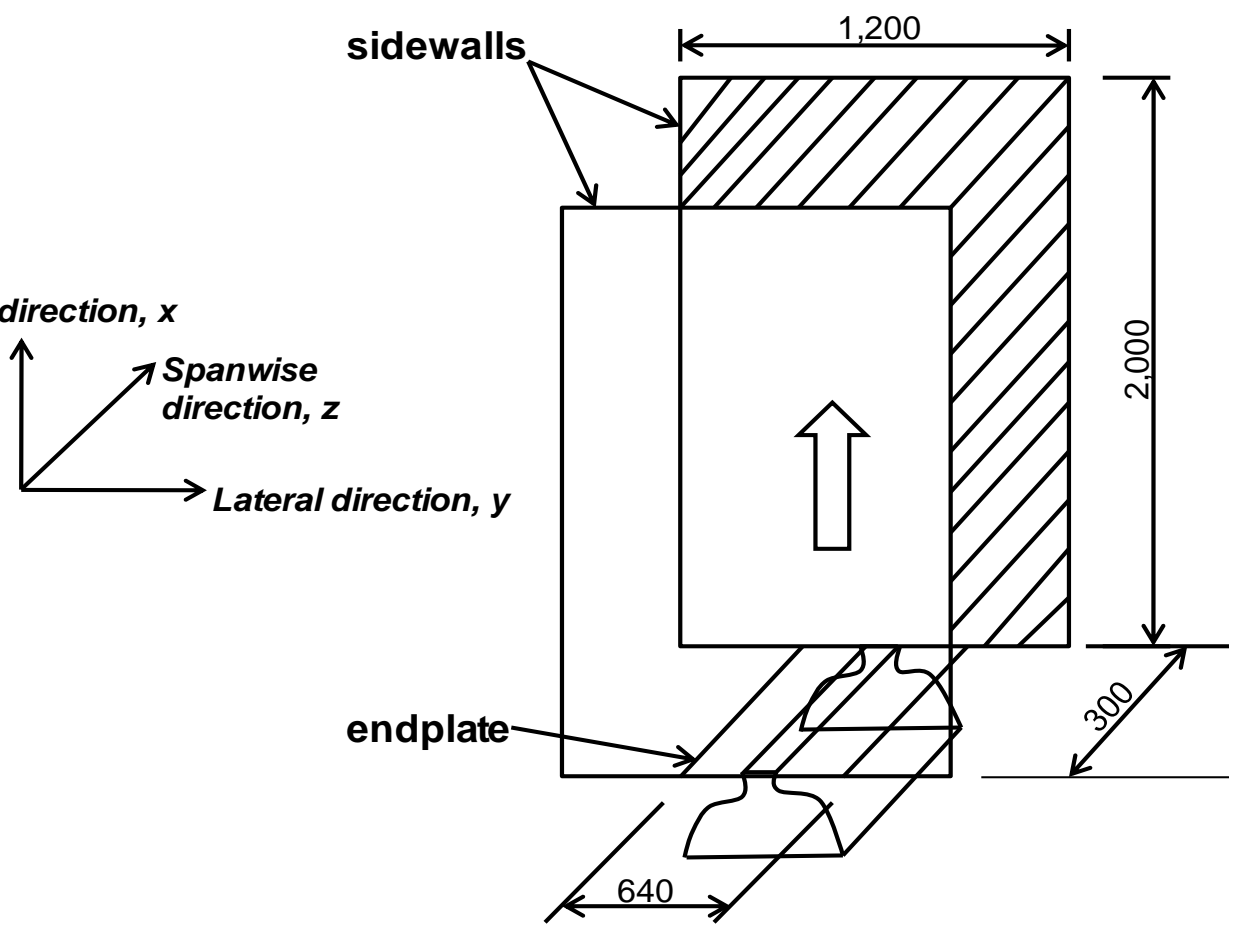

Figure 1.2: A schematic view of a rectangular nozzle with sidewalls and endplate. (Dimensions in this figure are the ones that used in the present investigation and all are in $\mathrm{mm})$.

Although sidewalls and/or endplates have been used in the above mentioned investigations at various initial conditions (e.g. different aspect ratio, Reynolds numbers, and nozzle shapes), few experiments have been devoted to explore their effects on turbulent jets issuing from rectangular nozzles (Hitchman et al., 1990, Papadakis \& Staiano, 1993, and Deo et al., 2007c).

Papadakis \& Staiano, (1993) tried to assess the influence of endplate on free plane jets (without sidewalls, NENS, WENS) of aspect ratio $A R=40$ at $R e=9,800$. Their study has investigated the mean velocity only up to a downstream location $x / D \leq 18$. 
Hitchman et al., (1990) and Deo et al., (2007c) investigated the effect of sidewalls on plane jets with and without endplate respectively. Both investigations used similar aspect ratio, $A R=60$ and nearly similar Reynolds number $\sim 7,000$. At this aspect ratio it is expected to have a longer region of 2-dimensional behaviour. Moreover, their measurements focused on the axial velocity component. In fact measuring two or all the velocity components is essential when evaluating the effects of endplates and/or sidewalls. According to our knowledge there is no available information on the combined effect of the endplate and sidewalls (WEWS).

\subsection{Contribution of the present thesis}

The objective of this investigation is to study the influence of the endplate and/or sidewalls on the near flow fields of a rectangular jet of aspect ratio, $A R=15$. To meet these goals, four different configurations directly compared, namely 1) jet without

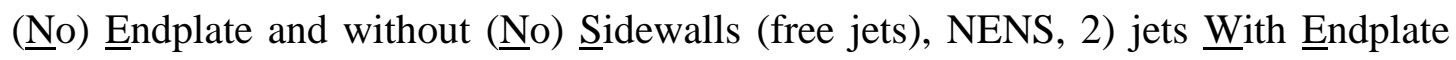
and without (№) Sidewalls, WENS, 3) jets without (№) Endplate and With

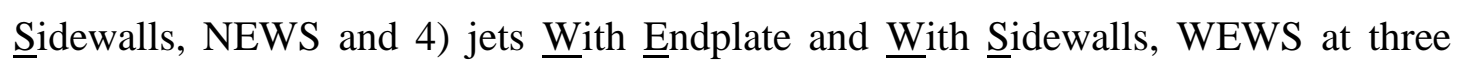
Reynolds numbers i.e. $R e_{D}=10,000,20,000$ and 30,000 (where $D$ is the width of the nozzle).

Using x-wire probe hot wire anemometry, the development of mean longitudinal and lateral velocities is studied to enable direct comparisons among the characteristics (e.g. spread rate, velocity decay, momentum, entrainment, self-similarity) of the four configurations. The turbulence field is also investigated to evaluate the influence of endplate and/or sidewalls on turbulent velocities, shear stresses and third moments. The present results are discussed and carefully compared to published data to investigate the possible contributions of the influences of the endplate and/or sidewalls.

\section{Main conclusions of the present work}

1) For the present jet, the effect of the endplate in the absence of the sidewalls is considered to be insignificant. 
2) In the presence of the sidewalls, the jets produce statistically different flow fields compared to the jets without sidewalls, as clearly indicated by the measured velocity field. The presence of the sidewalls seems to improve the two-dimensionality as indicated by the measured flow field e.g. formation of sharp peaks of the skewness factor at the edges. But again, their presence seems to alter the three-dimensional development of the mean streamwise velocity in the spanwise direction.

3) The presence of the sidewalls delays the turbulent streamwise velocity component to attain self-preservation in comparison to the jets without sidewalls.

\subsection{Open issues and further work}

The open issues left out of the present investigation along with recommendations for further work are given below:

1) In the present work, $x$-wire probe hot wire anemometry was used to measure the 2-dimensional flow fields of all configurations and no visualization of the flow field was made. Therefore, visualizing the flow fields of the four configurations is recommended for better insight and a clearer picture of the presence of the sidewalls/or endplates.

2) The velocity fields of the four configurations have been studied up to a downstream location of 35 nozzle widths only due to the limitations of the experimental set-up. Therefore it is may be recommended to study other locations beyond $x / D=35$.

3) In general the effects of sidewalls and endplate on a rectangular jet have to be investigated in a broader range of aspect ratios, inlet conditions, and Reynolds numbers. 


\subsection{Thesis outline}

Chapter 1 introduces some applications of turbulent rectangular jets followed by the physics of these jets. Next, the importance of the various initial and boundary conditions is highlighted. Main contributions of the present thesis followed by the open issues which are left out beside the recommended further work are also given.

Chapter 2 starts with brief introductions on turbulent flows, free shear flows, boundary layer flows, and turbulent jets. Next, the descriptions of the velocity flow fields of rectangular jets are given. A review on the formulation of the equation of motion, the integral momentum equation, and the entrainment hypothesis are also provided. Finally, various initial and boundary conditions of rectangular jets are defined.

Chapter 3 reviews the literature devoted to study the turbulent jets issuing from rectangular nozzles. The need of the present investigation is discussed based on the available information.

Chapter 4 begins with a description of the jet facility used in the present investigation. Following this, hot wire anemometry is introduced (i.e. constant temperature anemometry, working principle, noise, advantages, disadvantages, selection of hot wire probes, calibration, measuring the velocity components, a specification of the measuring system used in the present investigation, and the experimental uncertainty). Finally the experimental procedure is given.

Chapter 5 presents all results of this investigation. The effects of endplate and/or sidewalls on mean and turbulent velocity fields of the studied jets are discussed. Comparisons with previous studies available in the literature are also provided.

Chapter 6 presents the overall conclusion of the present thesis. 


\section{Chapter 2}

\section{THEORY}

\subsection{Turbulent flows}

Turbulent flows can be observed in many occasions although observer may or may not be aware about it. They can be identified in water flows in rivers or waterfalls, or in strong wind streams. Turbulent flows can also be found in many engineering and technological applications. In industries, chimneys release clouds of smoke to the environment. Turbulence is also present in invisible cases such as flows in pumps, compressors, pipe lines, around the airplanes, automobiles, ships etc. In the above mentioned examples, when the flow becomes unsteady, irregular, random and chaotic, and the motion of every eddy is unpredictable, then the flow is considered to be turbulent (Pope, 2002).

Turbulent flows enhance the ability to transfer momentum, energy and species of the fluid particles in comparison to laminar flow which is desirable for the mixing processes i.e. the demand usually is to have as rapid mixing as possible. Such improvement will reduce the impact of pollutant streams released from chimneys of industries into atmosphere, and for the mixing of various reactants in combustion devices (Pope, 2002).

\subsection{Free shear flows}

Free shear flows include all fluid flows which are free to develop without any effects from solid boundaries. They also include flows which originate through contact with solid surfaces e.g. flows next to body such as airplanes, cars. These flows can be considered without boundaries, at least to some extent (Bernard \& Wallace, 2002).

Figure 2.1 illustrates four types of free shear flows, these are, jets, mixing layer, wake, and boundary layer. In plane jet flows (Figure 2.1-a), fluid leaves the nozzle to 
larger area. The excess of jet's velocity compared to the surrounding is responsible for spreading the jet into that surrounding. In mixing layers, as illustrated in Figure 2.1-b, the fluid layers flows at different velocities which are brought together. The wake flows generally develop downstream of streamlined or bluff body (Figure 2.1c). In these flows, turbulence is formed around the body which is filled up in the wake region. Here, the mean velocity behind the object shows a defect in momentum compared to the outside region (Bernard \& Wallace, 2002). The last case is the boundary layer flow. In this case, fluid flows past a plate can form a boundary layer as shown in Figure 2.1-d. In such flows, a boundary layer is formed by the fluid on the plate. The velocity distribution is zero on the plate due to the no-slip condition and increasing gradually away from the plate (Douglas et al., 1995).

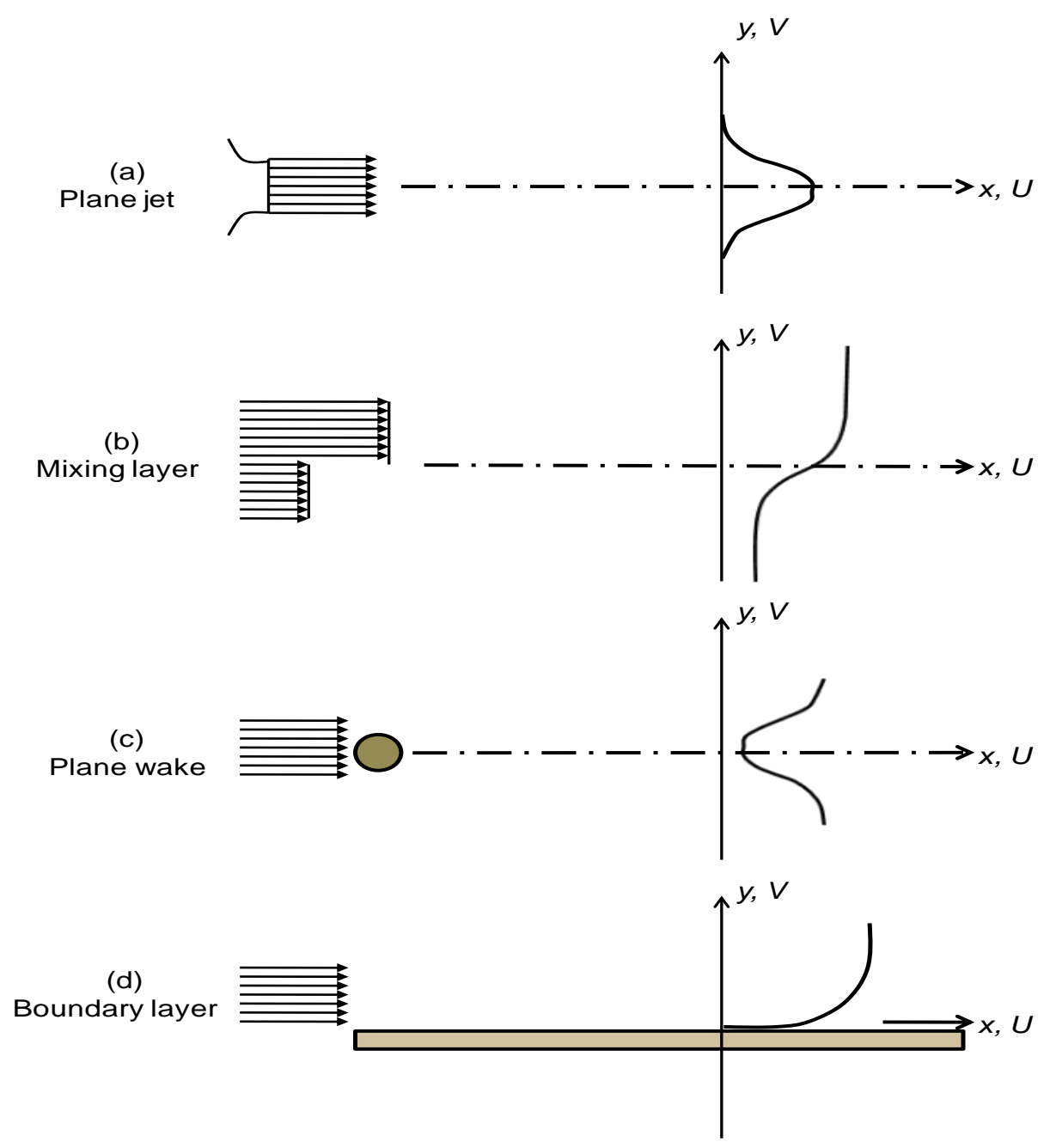

Figure 2.1: Illustrations of two dimensional flows. Redrawn after Pope, (2002). 


\subsection{Boundary layer flows}

If we consider a fluid which flows on a flat plate as shown in Figure 2.2 (i.e. along $x$ coordinate) with free stream velocity, $U_{\infty}$, the fluid will form adjacent layers on each other over that flat plate (i.e. normal to the plate, $y$-coordinate). The first layer on the flat plate will attain zero velocity due to the no-slip condition. This motionless layer will decelerate the neighboring layers due to the friction between fluid's particles of these two layers of different velocities. As a result of this process, this layer will slow down the molecules of the next layer. This mechanism will transfer from layer to layer until the velocity will be equal to free stream velocity, $U_{\infty}$. Therefore, the fluid's velocity can vary from zero at the plate to free stream velocity, $U_{\infty}$ at $y=\delta$. The flow region above the plate bounded $\delta$ where the effect of viscous shear forces caused by fluid viscosity is felt, is termed as velocity boundary layer (or boundary layer). The boundary layer thickness is defined as the distance from the flat plate where $y=0$ to distance y at $U=0.99 U_{\infty}$.

It is may be interesting to differentiate between the viscous and inviscid flow regions regarding the flow over a flat plate. If we consider the imaginary line where $U=0.99$ Us as base, we will find that the region under that line where the viscous forces are considered (i.e. boundary layer) is termed as a viscous flow region while above that imaginary line, the region is termed as invicid flow. In the invicid flow region, the frictional effects are negligible and the velocity will remain nearly unchanged (Çengel, 1997).

In the boundary layer region, the faster fluid layer will drag along the slower layer due to the friction between the two layers which will result in drag force (or friction) on it. The drag force per unit area is known as the shear stress, $\tau$. Experimental studies have shown that the shear stress is proportional to the velocity gradient. The shear stress at plate surface can be expressed by

$$
\tau=\mu d u / d y
$$


where $\mu$ is the dynamic viscosity of the fluid which is a measure of the flow resistance. Its unit is $\mathrm{kg} /(\mathrm{m} . \mathrm{s})$ or equivalently N.s/ $\mathrm{m}^{2}$. The viscosities of liquids decrease with temperature whereas for the gases, it increases (Çengel, 1997)

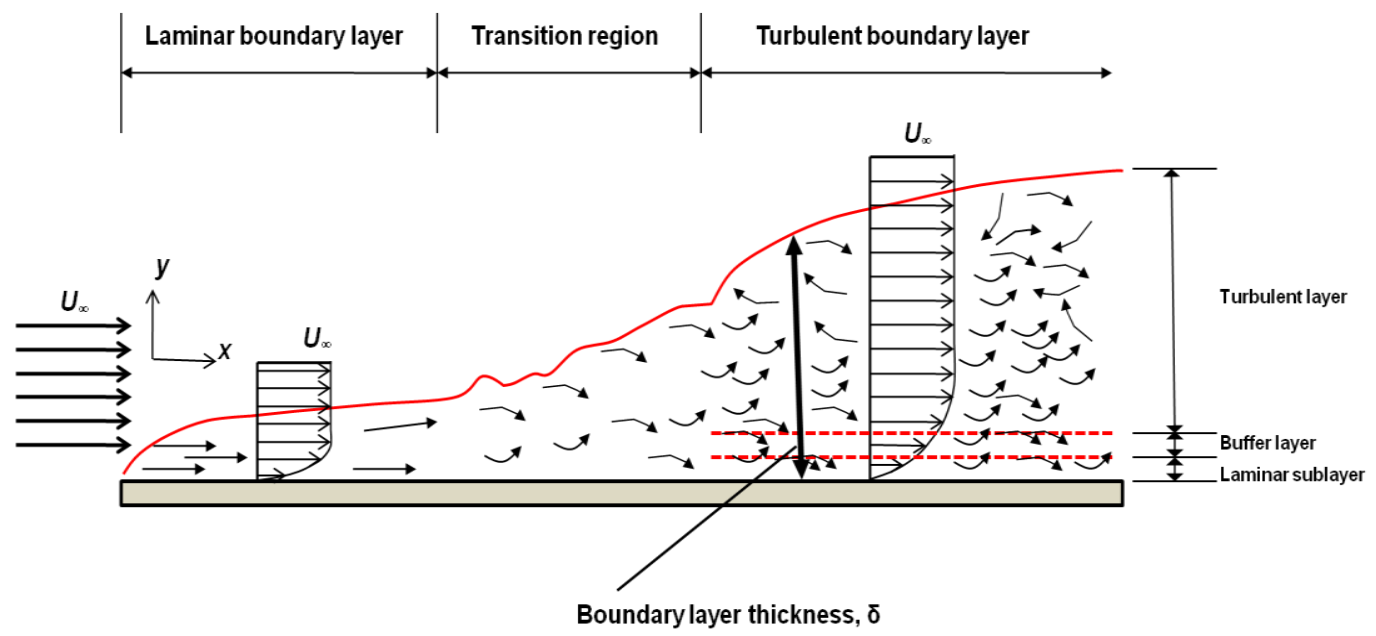

Figure 2.2: The development of boundary layer for flow over a flat plate and different flow regimes. Redrawn after Çengel, (1997).

\subsection{Turbulent jets}

When the fluid moves, a shear layer is formed between the adjacent layers. The flow of these layers is termed as a jet. These jets may be moving in the same or opposite directions. The instabilities of these shear layers lead to the formation of eddies which move randomly. These eddies are responsible for the exchange of matters (e.g. momentum, heat and constituents) among the neighboring jets; i.e. there is a transverse transfer of momentum, heat, and constituents (Abramovich, 1963).

A finite thickness region is formed between two jets, responsible for the continuous distribution of velocity, temperature, and species concentration. This region is called turbulent jet boundary layer, in rare cases, it can be laminar at very low Reynolds numbers. Boundary layer in a simple case can be found when a jet flows in a moving medium at fixed velocity. In this case the thickness of the boundary layer in the initial stages is equal to zero. And as the jet develops, the thickness of the boundary layer increases, carrying the fluid's particles which results in spreading of the jet (Abramovich, 1963). 
When the jet leaves its exit into an ambient atmosphere, its free surface is exposed to the atmosphere; hence the pressure of the jet itself is an atmospheric pressure. However, the pressure at exit can be also different from the atmospheric pressure. Such boundary condition is maintained only if we take into account the surface tension effect which is usually not considered, or in supersonic jet which can separate itself from atmosphere with expansion or compression waves (White, 1994).

Figure 2.3 shows a round jet facility with the defining coordinates. A fluid is discharged from a nozzle with diameter, $D_{\mathrm{r}}$ producing a nearly constant initial velocity profile with velocity $U$. The flow is axisymmetric in the developed region hence statistics depends on the axial and radial coordinates $(x$, and $r$ ) but not on circumferential coordinate, $\theta$ and time. The velocity components in the $x, r$, and $\theta$ coordinate directions are denoted by $U, V$, and $W$ (Pope, 2002).

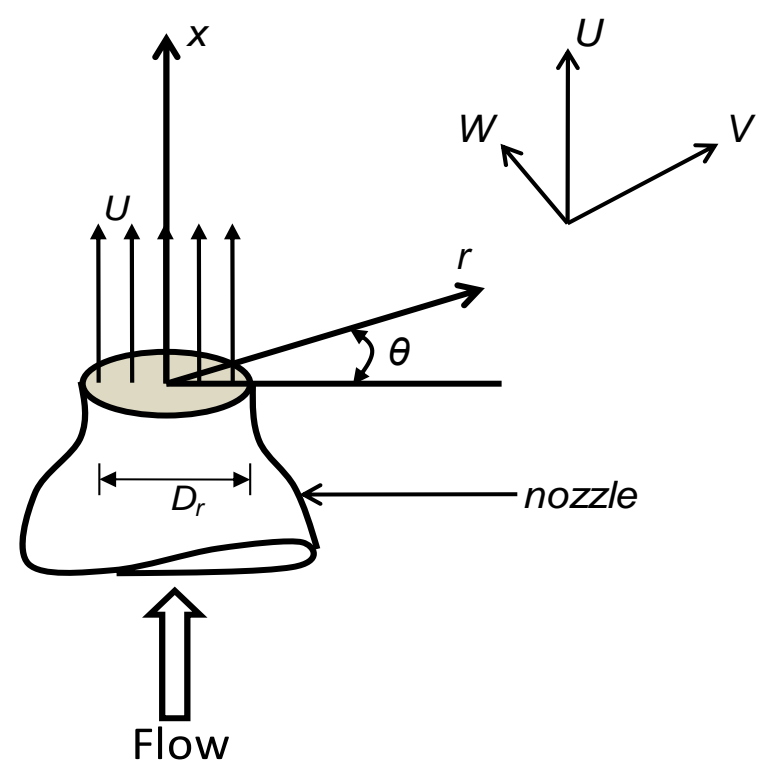

Figure 2.3: Illustration of the round jet facility and coordinate. Redrawn after Pope, (2002).

Figure 2.4 shows non-circular nozzles shapes as well as circular one used by Mi et al., 2000 to produce different jets. In the present investigation we will deal only with the turbulent jets issuing from a rectangular nozzle. 


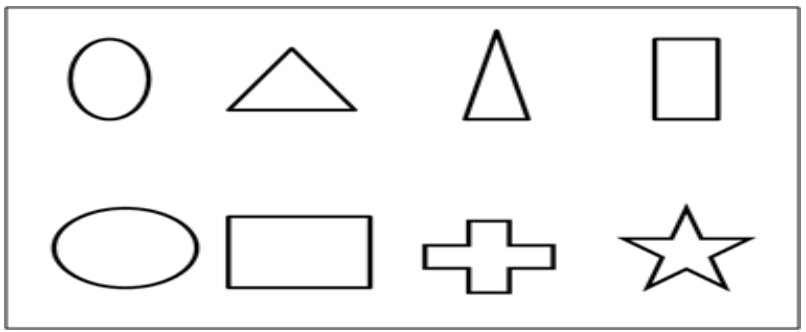

Figure 2.4: Orifice shapes used by Mi et al., (2000).

\subsection{Turbulent jets issuing from rectangular nozzles}

Turbulent jets issuing from rectangular nozzles are free shear flows driven by the momentum introduced at the exit. As soon as the jet leaves the nozzle, the jet will start entraining the ambient fluid. The momentum will be exchanged with ambient fluid thus the jet will spread. Further downstream the mixing region will be wider penetrating the centerline after that the centerline velocity will decay and the turbulence intensities will grow. Large vortical structures are formed in the shear layer between the jet and its surrounding. These vortices carry out the irrotational ambient fluid and hence induce mixing. As soon as the vortices move downstream, they will grow, become larger and interact with each other. As these vortices will move further downstream, secondary instabilities will break them up (Namer \& Ötügen, 1988).

Plane or two dimensional jets (i.e. the jet entrains fluid only in axial and lateral directions), are produced from high aspect ratio nozzles. Their velocity fields are two dimensional. Pope, (2002) stated that aspect ratio must be larger than 50 to obtain two dimensional flow fields without any assistance from the sidewalls. Whereas in rectangular or three dimensional jets, smaller aspect ratios are used (Rajaratanam, (1976). In these jets, the velocity fields will be axisymmetric after the two dimensional region and the length of the later region was found to be a function of the aspect ratio (e.g. Rajaratanam, 1976, Krothapalli et al., 1981, Deo et al., 2007a). The velocity flow fields of a rectangular jet are shown in Figure 1.1. 


\subsubsection{Description of the velocity field}

\subsubsection{Mean velocity fields}

\section{a) Mean streamwise velocity}

Considering the lateral profiles, in the near fields, the mean streamwise velocities, $U$ will be nearly similar (i.e. $\mathrm{U}_{\mathrm{c}} \sim 0.98 \mathrm{U}_{0}$, Deo et al., 2007a) forming a top-hat velocity profile (see Figure 5.2). This region is known as the potential core region. It is used as a measure for the mixing rate between the jet and its surrounding fluid. In other word, a shorter potential core length, $x_{p}$ implies higher mixing levels (e.g. Quinn, 1992, Deo et al., 2007a). Several investigators have found that the length of the jet's potential core region is influenced by the various initial and boundary conditions (e.g. Deo et al., 2007a, Deo et al., 2007b, Deo et al., 2007c, Deo et al., 2008). The potential core region ends when the two shear layers from the short sides of the nozzle meet (e.g. krothapalli et al., 1981) and by going further downstream from the nozzle exit, the shape of the mean streamwise profiles will gradually take a bell shape. These profiles will attain similarity after some downstream locations from the exit (see Figure 5.2).

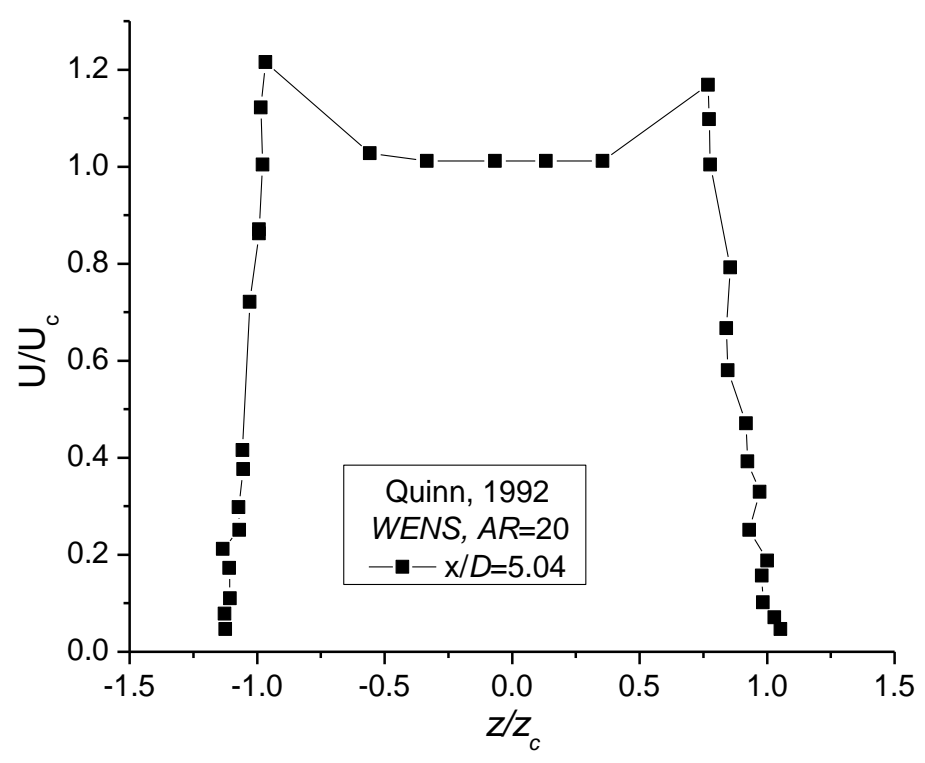

Figure 2.5: Spanwise profiles of mean streamwise velocity of Quinn, (1992). 
The development of the mean streamwise velocity in the spanwise direction (i.e. along the jet) of the present investigation (for NENS) is shown in Figure 5.3. As one can see, uniform mean streamwise velocity distributions are observed, but further downstream undulations are appeared which is more evidenced in the furthest measured location. Other authors (e.g. Marsters, 1980, Tsuchiya et al., 1986, Quinn, 1992) have observed these undulations which were referred as saddle back shape mean streamwise velocity profiles. The saddle back velocity profiles of Quinn, 1992 is shown in Figure 2.5. It is also of interest to mention that the present investigation have revealed that the presence of the sidewalls will result in different mean streamwise velocity profiles in the spanwise direction, in comparison to the free jet case (Figure 5.3).

\section{b) Mean lateral velocity}

From the present investigation, the lateral development of the mean lateral velocity, $V$ in turbulent jets issuing from rectangular nozzle is shown in Figure 5.5a. Zero values are at the centre at all downstream locations, extending in a larger area at the closest to the exit locations, depicting the presence of the potential core. The mean lateral velocity is responsible for transferring longitudinal momentum to larger area and hence responsible for the spreading the jet.

\section{c) Mean spanwise velocity}

The magnitudes of mean spanwise velocities are usually very small. The shape and development of the mean spanwise velocity, $W$ in the spanwsie direction of the jet of krothapalli et al., (1981) at various downstream locations is shown in Figure 2.6. 


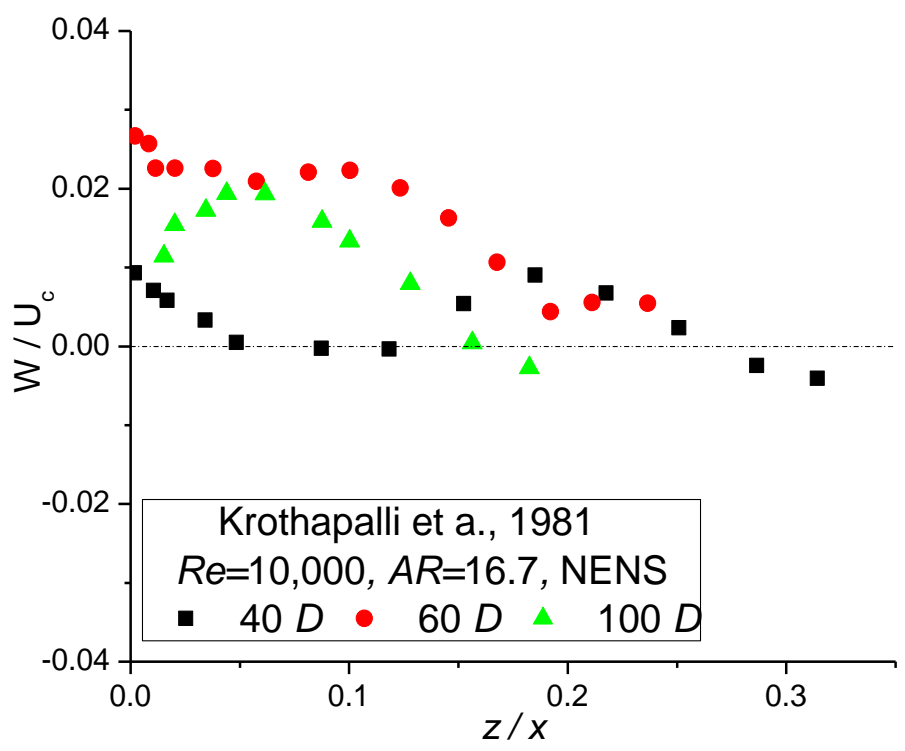

Figure 2.6: Development of mean spanwise velocity in the spanwise direction. Data extracted from Krothapalli et al., (1981).

\section{d) Spread of rectangular jets}

Experimentally, the spreading of rectangular jets is referred as the growth of these jets. The spreading of turbulent rectangular jets across the small dimension of the nozzle is larger than that in the large dimension. In experiments, the growth (or spread) of a rectangular jet across the minor dimension of the nozzle can be estimated using the lateral profiles of the mean velocity fields to derive the values of $y_{c}$ which is the location in $y$ axis where the velocity is half of the mean streamwise velocity at the centerline, (see Figure 1.1 and Figure 2.7). Similarly, the spread across the major dimension of the nozzle can be estimated by deriving the values of $z_{c}$ (which is the location in $z$ axis where the velocity is half of the mean streamwise velocity at the centerline) (see Figure 1.1), from the spanwise distributions of the mean streamwise velocity. Experimental investigations have shown that the value of $y_{c}$ varies linearly after some downstream locations from the nozzle's exit (e.g. Deo et al., 2007a). Using the value of $y_{c}$, the spread rate of the jet, $A_{1}$ and the spread virtual origin, $A_{2}$ can be estimated using the following relationship:

$$
y_{d} / D=A_{l}\left[x / D+A_{2}\right]
$$




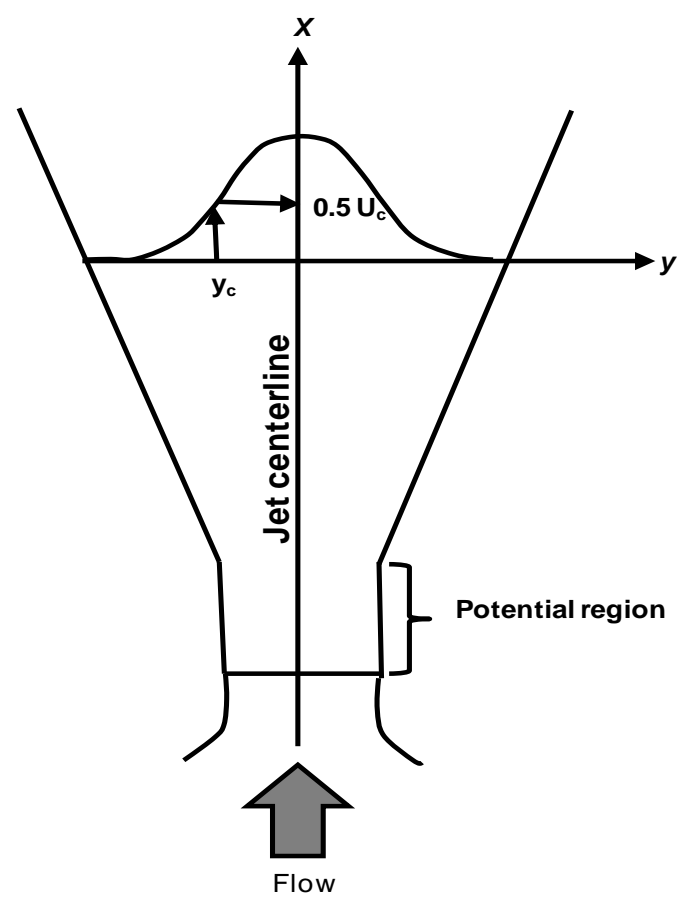

Figure 2.7: Illustration of jet's development across the minor dimension of a nozzle indicating the location of $y_{c}$. Redrawn after modification from Deo, (2005).

The spread rate and the spread virtual origin of rectangular jets have been found to be influenced by the different boundary and initial conditions (e.g. Deo et al., 2007a, Deo et al., 2007b, Deo et al., 2007c, Deo et al., 2008).

\section{e) Decay of centerline mean velocity}

The far field variation of the centerline velocity decay is found to follow a linear behavior of the form:

$$
\left(U_{0} / U_{c}\right)^{2}=B_{1}\left[x / D+B_{2}\right]
$$

Where $U_{0}$ is the mean streamwise velocity at the exit plane, $U_{c}$ is the local centerline mean velocity, the slope, $B_{1}$ is a measure of the centreline mean velocity decay rate and $B 2$ is the jet's virtual origin. The values of $B 1$ and $B 2$ have been found to depend on the various initial and boundary conditions (e.g. Deo et al., 2007a, Deo et al., 2007b, Deo et al., 2007c, Deo et al., 2008). 


\section{f) Type of initial boundary layer}

The type of initial boundary layer (i.e. laminar or turbulent) of rectangular jets can be identified by estimating the shape factor, $H$ (e.g. Hussain \& Clark, 1977, Deo, 2005), which is the ratio of the displacement thickness, $\delta_{l}$ to the momentum thickness, $\theta_{m}$ (i.e. $H=\delta_{1} / \theta_{m}$ ). The values of $\delta_{1}$ and $\theta_{m}$ can be estimated from the lateral mean streamwise velocity profiles at the exit plane using the following relations

$$
\begin{aligned}
& \delta_{1}=\int_{\mathrm{y}=0}^{\mathrm{y}=\infty}\left(1-\frac{\mathrm{U}}{\mathrm{U}_{0}}\right) d y \\
& \theta_{m}=\int_{\mathrm{y}=0}^{\mathrm{y}=\infty} \frac{\mathrm{U}}{\mathrm{U}_{0}}\left(1-\frac{\mathrm{U}}{\mathrm{U}_{0}}\right) d y
\end{aligned}
$$

\subsubsection{Turbulent velocity fields}

\section{a) Turbulent velocities}

In turbulent flows, fluctuations will lead the velocity to rapidly vary in a random function of time and space (White, 1994). If we consider a turbulent velocity, $u$ ' in e.g. streamwise direction, then $u^{\prime}$ is defined as the deviation of $U$ from its average value $u\left(u^{\prime}=u-U\right)$ as shown in Figure 2.8.

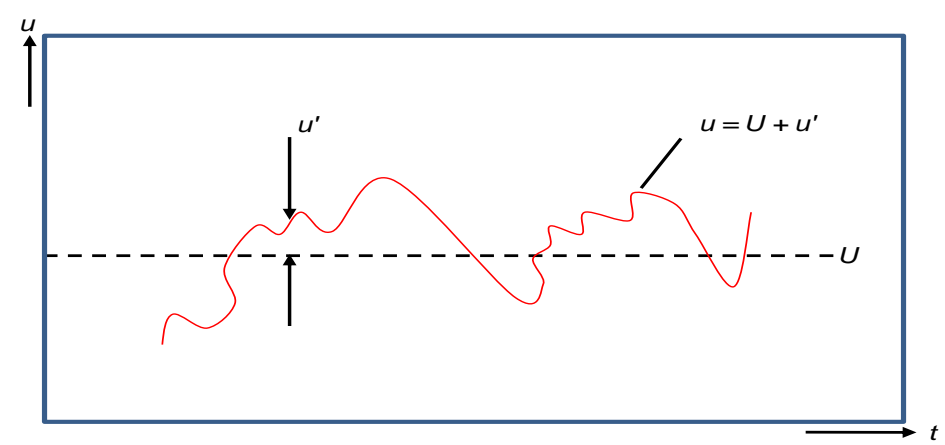

Figure 2.8: Definition of mean and fluctuating turbulent of velocity. Redrawn after White, (1994).

Therefore, identifying the turbulent velocities in rectangular jets is very essential to understand their evolution. The lateral profiles of turbulent streamwise and lateral velocities are shown in Figure 5.11 and Figure 5.16 respectively for different jet 
configurations. The development of the turbulent spanwise velocity in lateral direction of Cavo et al., (2007) is shown in Figure 2.9.

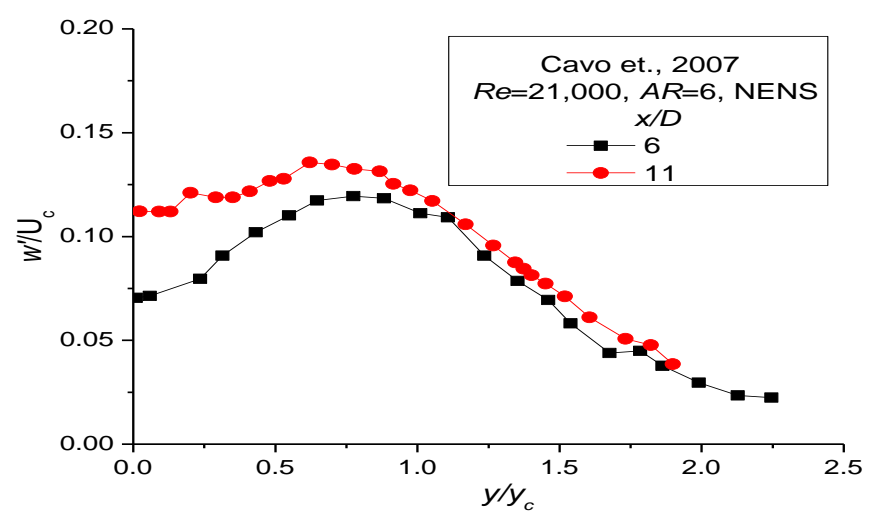

Figure 2.9: Lateral profiles of the turbulent spanwise velocity at different downstream locations. Data extracted from Cavo et., (2007).

\subsubsection{Self-similarity concept}

Self-similarity is a very important concept that arises in studying the turbulent flows (Pope, 2002). As long as one experiment is concerned, it is expected that after some downstream locations from the exit, the profiles of both mean and turbulent velocities (streamwise, lateral, and spanwise) when normalized to a particular parameter, will collapse on the next other profiles and hence attaining self similarity. Experimental investigations have shown that the location where the distributions of the velocity components (either mean or turbulent) first attain self similarity varies in the axial direction. Also this location is found to be a function of various initial and boundary conditions (e.g. Deo et al., 2007a, Deo et al., 2008). As an example, in the present investigation, the profiles of mean streamwise velocity in the absence of the sidewalls (NENS, WENS) attained similarity at a location equal or more than five nozzle widths (see Figure 5. 2a and b) while the profiles of the turbulent streamwise velocity were self-similar at a downstream location equal or greater than 15 nozzle widths (Figure 5.12a and b). But though the presence of the sidewalls (NEWS, WEWS) did not modify the location where mean streamwise velocity profiles became self-similar (Figure 5.2c and d), sidewalls 
have delayed the profiles of the turbulent streamwise velocity to attain similarity (Figure 5.12c and d).

\subsection{Related equations}

\section{a) Equation of motion}

In this section, the equations of motion for the turbulent plane jet are discussed (Rajaratanam, 1976, Chapter 1).

In a turbulent flow, the velocity of every particle that passed through a point at any given time is given by $V=(u, v$ and $w)$, Then one would find the actual velocity fluctuating about a mean value:

$$
u=U+u^{\prime}, v=V+v^{\prime} \text { and } w=W+w^{\prime}
$$

where, $u, v$ and $w$ are the actual velocities, $U, V, W$ are mean velocities and $u^{\prime}$, $v^{\prime}$ and $w^{\prime}$ are the fluctuating velocities in the $x, y, z$ directions respectively (Figure $1.1)$

$$
\begin{aligned}
\frac{\partial u}{\partial t}+u \frac{\partial u}{\partial x}+ & v \frac{\partial u}{\partial y}+w \frac{\partial u}{\partial z}=-\frac{1}{\rho} \frac{\partial p}{\partial x}+v\left(\frac{\partial^{2} u}{\partial x^{2}}+\frac{\partial^{2} u}{\partial y^{2}}+\frac{\partial^{2} u}{\partial z^{2}}\right) \\
- & \left(\frac{\partial \overline{u^{\prime}}}{\partial x}+\frac{\partial \overline{u^{\prime} v^{\prime}}}{\partial y}+\frac{\partial \overline{u^{\prime} w^{\prime}}}{\partial z}\right) \\
\frac{\partial v}{\partial t}+u \frac{\partial v}{\partial x}+ & v \frac{\partial v}{\partial y}+w \frac{\partial v}{\partial z}=-\frac{1}{\rho} \frac{\partial p}{\partial y}+v\left(\frac{\partial^{2} v}{\partial x^{2}}+\frac{\partial^{2} v}{\partial y^{2}}+\frac{\partial^{2} v}{\partial z^{2}}\right) \\
- & \left(\frac{\partial \overline{u^{\prime} v^{\prime}}}{\partial x}+\frac{\partial \overline{v^{\prime 2}}}{\partial y}+\frac{\partial \overline{v^{\prime} w^{\prime}}}{\partial z}\right) \\
\frac{\partial w}{\partial t}+u \frac{\partial w}{\partial x}+ & v \frac{\partial w}{\partial y}+w \frac{\partial w}{\partial z}=-\frac{1}{\rho} \frac{\partial p}{\partial y}+v\left(\frac{\partial^{2} w}{\partial x^{2}}+\frac{\partial^{2} w}{\partial y^{2}}+\frac{\partial^{2} w}{\partial z^{2}}\right) \\
- & \left(\frac{\partial \overline{u^{\prime} w^{\prime}}}{\partial x}+\frac{\partial \overline{v^{\prime} w^{\prime}}}{\partial y}+\frac{\partial \overline{w^{\prime 2}}}{\partial z}\right)
\end{aligned}
$$

The continuity equation is written as

$$
\frac{\partial u}{\partial x}+\frac{\partial v}{\partial y}+\frac{\partial w}{\partial z}=0
$$


Where $x$-axis defines the streamwise direction, $y$-axis defines the lateral direction, and $z$-axis defines the spanwise direction. In the following derivations, $u, v, w$ and $u^{\prime}, v^{\prime}, w^{\prime}$ are the mean and turbulent velocities in the streamwise, lateral and spanwsie direction. It should be mentioned that throughout this thesis except this part (i.e. these derivations), the mean velocities in the streamwise, lateral and spanwise directions are denoted as $U, V$, and $W$ respectively. Here, $p$ is the mean pressure at any point, $v$ is the kinematic viscosity, and $\rho$ is the mass density of the fluid and $t$ is the time variable.

If the mean flow is two-dimensional, $w=0, \partial / \partial \mathrm{z}$ of any mean quantity is zero, $u^{\prime} w^{\prime}=$ $0, v^{\prime} w^{\prime}=0$ and since the mean flow is steady $\partial u / \partial \mathrm{t}=0$ and $\partial v / \partial \mathrm{t}=0$. Further, since the transverse extent of the flow is small, $u$ is generally much larger than $v$ in a large portion of the jet and velocity and stress gradients in the $y$-direction are much larger than those in the $x$-direction.

With these considerations, the equations of motion could be shown to reduce to the form:

$u \frac{\partial u}{\partial x}+v \frac{\partial u}{\partial y}=-\frac{1}{\rho} \frac{\partial p}{\partial x}+v \frac{\partial^{2} u}{\partial y^{2}}-\frac{\partial \overline{u^{\prime} v^{\prime}}}{\partial y}-\frac{\partial \overline{u^{\prime 2}}}{\partial x}$

$0=-\frac{1}{\rho} \frac{\partial p}{\partial y}-\frac{\partial \overline{v^{\prime 2}}}{\partial y}$

$\frac{\partial u}{\partial x}+\frac{\partial v}{\partial y}=0$

Integrating (2.12) with respect to $\mathrm{y}$ from $\mathrm{y}$ to a point located outside the jet, we obtain:

$p=p_{\infty}-\rho \overline{v^{\prime 2}}$

where $p_{\infty}$, is the pressure outside the jet. Differentiating the above equation and substituting in (2.11), we get: 
$u \frac{\partial u}{\partial x}+v \frac{\partial u}{\partial y}=-\frac{1}{\rho} \frac{d p_{\infty}}{d x}+v \frac{\partial^{2} u}{\partial y^{2}}-\frac{\partial \overline{u^{\prime} v^{\prime}}}{\partial y}-\frac{\partial}{\partial x}\left(\overline{u^{\prime 2}}-\overline{v^{\prime 2}}\right)$

The last term in the above equation is very small and can be neglected. Hence we obtain the reduced equations of motion as:

$u \frac{\partial u}{\partial x}+v \frac{\partial u}{\partial y}=-\frac{1}{\rho} \frac{d p_{\infty}}{d x}+v \frac{\partial^{2} u}{\partial y^{2}}-\frac{\partial \overline{u^{\prime} v^{\prime}}}{\partial y}$

and,

$\frac{\partial \mathrm{u}}{\partial \mathrm{x}}+\frac{\partial \mathrm{v}}{\partial \mathrm{y}}=0$

where $p_{\infty}$, is simply written as $p$ for convenience. In (2.15), we could rewrite the last two terms as:

$\frac{1}{\rho} \frac{\partial}{\partial y}\left(\mu \frac{\partial u}{\partial y}\right)+\frac{1}{\rho} \frac{\partial}{\partial y}\left(-\rho \overline{u^{\prime} v^{\prime}}\right)=\frac{1}{\rho} \frac{\partial}{\partial y}\left(\tau_{1}+\tau_{t}\right)$

where $\tau_{1}$ and $\tau_{t}$ are, respectively, the laminar and turbulent shear stresses and $\mu$ is the coefficient of dynamic viscosity. In free turbulent flows, due to the absence of solid boundaries, $\tau_{\mathrm{t}}$ is much larger than $\tau_{1}$ and hence it is reasonable to neglect $\tau_{1}$ and rewrite (2.15) as:

$u \frac{\partial u}{\partial x}+v \frac{\partial u}{\partial y}=-\frac{1}{\rho} \frac{d p}{d x}+\frac{1}{\rho} \frac{\partial \tau_{t}}{\partial y}$

Further, because in a large number of practical problems the pressure gradient in the axial direction is negligibly small and also to study the jet under relatively simpler conditions, let us set $d p / d x=0$. Then (2.16) and (2.13) become:

$u \frac{\partial u}{\partial x}+v \frac{\partial u}{\partial y}=\frac{1}{\rho} \frac{\partial \tau_{t}}{\partial y}$

$\frac{\partial u}{\partial x}+\frac{\partial v}{\partial y}=0$ 
which are the well-known equations of motion for the plane turbulent free jet with a zero pressure gradient in the axial direction.

\section{b) The integral momentum equation}

For the plane turbulent jet issuing into a large stagnant environment and expanding under zero pressure gradient, since there is no external force involved, it is easy to see that the momentum of the jet in the axial direction is preserved. Let us now derive this criterion in an elegant manner, and this procedure will be helpful when we study more complex situations. Multiplying (2.17) by $\rho$ and integrating from $y=0$ to $y=\infty$, we have:

$\rho \int_{0}^{\infty} u \frac{\partial u}{\partial x} d y+\rho \int_{0}^{\infty} v \frac{\partial u}{\partial y}=\int_{0}^{\infty} \frac{\partial \tau}{\partial y} d y$

Let us now consider the different terms of the above equation.

$$
\begin{aligned}
\rho \int_{0}^{\infty} u \frac{\partial u}{\partial x} d y & =\frac{1}{2} \int_{0}^{\infty} \frac{\partial}{\partial x}\left(\rho u^{2}\right) d y=\frac{1}{2} \frac{d}{d x} \int_{0}^{\infty} \rho u^{2} d y \\
\rho \int_{0}^{\infty} v \frac{\partial u}{\partial y} d y & =\rho\left(|u v|_{0}^{\infty}-\int_{0}^{\infty} u \frac{\partial v}{\partial y} d y\right) \\
& =\rho\left(\int_{0}^{\infty} u \frac{\partial u}{\partial x} d y\right)
\end{aligned}
$$

(by Liebnitz rule)

Since $\mathrm{y}=0: u=u_{m}, \mathrm{v}=0$

And for $\mathrm{y} \rightarrow \infty ; \mathrm{u}=0, \mathrm{v}=\mathrm{v}_{\mathrm{e}}$

Where $v_{\mathrm{e}}$ is finite quantity known as entrainment velocity. Thus

$\rho \int_{0}^{\infty} v \frac{\partial u}{\partial y}=\frac{1}{2} \frac{d}{d x} \int_{0}^{\infty} \rho u^{2} d y$

The left-hand side of (2.19) becomes equal to:

$\frac{d}{d x} \int_{0}^{\infty} \rho u^{2} d y$

Considering the remaining term:

$\int_{0}^{\infty} \frac{\partial \tau_{t}}{\partial y} \mathrm{dy}=|\tau|_{0}^{\infty}=\tau(\infty)-\tau(0)=0$ 
since $\tau(0)=0$ from considerations of symmetry and it is reasonable to assume $\tau(\infty)$ is zero. Hence (2.19) becomes:

$\frac{d}{d x} \int_{0}^{\infty} \rho u^{2} d y=0$

Equation (2.20) tells us that the rate of change of the momentum flux in the $x$ direction is zero; that is the moment flux in the $x$-direction is conserved (or preserved).

Integrating (2.20):

$2 \int_{0}^{\infty} \rho u^{2} d y=\mathrm{M}_{0}$

The momentum flux $M_{0}$, is an important physical quantity controlling the behavior of the plane jet.

\section{c) The integral energy equation}

Let us multiply the first equation of motion by $\rho u$ and integrate it with respect to $y$ from $y=0$ to $y=\infty$. We get:

$$
\int_{0}^{\infty} \rho u^{2} \frac{\partial u}{\partial x} d y+\int_{0}^{\infty} \rho u v \frac{\partial u}{\partial x} d y=\int_{0}^{\infty} u \frac{\partial \tau}{\partial y} d y
$$

Let $E=\rho u^{2} / 2$, be the kinetic energy per unit volume.

$\int_{0}^{\infty} \rho u^{2} \frac{\partial u}{\partial x} d y=\rho \int_{0}^{\infty} u \frac{\partial}{\partial x} \frac{\rho u^{2}}{2} d y=\int_{0}^{\infty} u \frac{\partial E}{\partial x} d y$

$\int_{0}^{\infty} \rho u v \frac{\partial u}{\partial y} d y=\int_{0}^{\infty} v \frac{\partial E}{\partial y} d y$

$\int_{0}^{\infty} \rho u 2 \frac{\partial u}{\partial x} d y+\int_{0}^{\infty} \rho u v \frac{\partial u}{\partial y} d y=\int_{0}^{\infty}\left(u \frac{\partial E}{\partial x}+v \frac{\partial E}{\partial y}\right) d y=\int_{0}^{\infty}\left(\frac{D E}{D t}\right) d y$ 
where $\mathrm{D} / \mathrm{Dt}$ stands for the particle derivative and $\mathrm{DE} / \mathrm{Dt}$ is the total rate of change of the kinetic energy.

$\int_{0}^{\infty} u \frac{\partial \tau}{\partial y} d y=|u \tau|_{0}^{\infty}-\int_{0}^{\infty} \tau \frac{\partial u}{\partial y} d y=-\int_{0}^{\infty} \tau \frac{\partial u}{\partial y} d y$

$\tau \partial u / \partial y$ is the rate of production of turbulence, by the Reynolds shear stress working on the mean velocity gradient. We have:

$\int_{0}^{\infty} \frac{D E}{D t} d y=-\int_{0}^{\infty} \tau \frac{\partial u}{\partial y} d y$

which says that the rate of decrease of the kinetic energy is equal to the rate at which turbulence is produced. For our present purposes, we will rewrite the above equation in a slightly different form.

$$
\begin{aligned}
\int_{0}^{\infty} u \frac{\partial E}{\partial x} d y & =\int_{0}^{\infty} u \frac{\partial}{\partial x} \frac{\rho u^{2}}{2} d y \\
& =\left[\frac{\partial}{\partial x}\left(\frac{\rho u^{2}}{2} u\right)-\frac{\rho u^{2}}{2} \frac{\partial u}{\partial x}\right] d y \\
\int_{0}^{\infty} v \frac{\partial E}{\partial y} d y & =\int_{0}^{\infty} v \frac{\partial}{\partial y} \frac{\rho u^{2}}{2} d y \\
& =\left|v \frac{\rho u^{2}}{2}\right|_{0}^{\infty}-\int_{0}^{\infty} \frac{\rho u^{2}}{2} \frac{\partial v}{\partial y} \mathrm{dy}=\int_{0}^{\infty} \frac{\rho u^{2}}{2} \frac{\partial u}{\partial x} d y
\end{aligned}
$$

Adding the above two expressions:

$\int_{0}^{\infty} u \frac{\partial E}{\partial x} d x+\int_{0}^{\infty} v \frac{\partial E}{\partial y} d y=\int_{0}^{\infty} \frac{\partial}{\partial x}\left(\frac{\rho u^{2}}{2} \mathrm{u}\right) \mathrm{dy}=\frac{d}{d x} \int_{0}^{\infty} \frac{\rho u^{2}}{2} u d y$

We could now write:

$\frac{d}{d x} \int_{0}^{\infty} \frac{\rho u^{2}}{2} u d y=-\int_{0}^{\infty} \tau \frac{\partial u}{\partial y} d y$ 
We can see from equation 2.24 that the rate of decrease of the kinetic energy flux is equal to the rate at which turbulence is produced.

\section{d) Entrainment hypothesis}

If $m$ is the rate of (forward) flow for unit length at any section of the jet:

$m=2 \int_{0}^{\infty} u d y$

If $\mathrm{m}$ is the flow from the nozzle, it is known from experiments that $m / m_{o}$ is greater than unity and assumes very large values as $x$ becomes large. That means, the jet entrains a considerable amount of the surrounding fluid as it travels forward. We now write:

$\frac{d m}{d x}=2 \frac{d}{d x} \int_{0}^{\infty} u d y=2 v_{e}$

Where $v_{e}$ is the so-called entrainment velocity.

\subsection{Initial and boundary conditions of rectangular jets}

\section{a) Reynolds number}

Experimental investigations have shown that the various characteristics of a jet (e.g. growth and decay rates, mass and momentum flow rates, turbulent flow fields) depend on Reynolds number (Namer \& Ötügen, 1988, Deo et al., 2008). For jet issuing from a rectangular nozzle, the jet exit Reynolds number, $R e$ is defined using the nozzle opening width $D$ (minor dimension of the nozzle, Figure 1.1), centerline mean velocity at the exit, $U_{0}$ and kinematic viscosity, $v$ of the tested fluid using the expression

$$
R e_{D}=U_{0} D / v
$$




\section{b) Aspect ratio}

The aspect ratio, $A R$ of a jet issuing from a rectangular nozzle is the ratio of the long side of the nozzle, $L$ to the short side of the nozzle, $D$ (see Figure 1.1)

$$
A R=L / D
$$

Aspect ratio plays a very important role in determining the characteristics of a jet issuing from rectangular nozzle. Therefore, much effort was given to study the influence of this parameter (e.g. Quinn, 1992, Deo et al., 200a).

\section{c) Nozzle type}

However turbulent rectangular jets are produced from rectangular openings, still there is a difference among these rectangular openings as long as the exit shape is considered. The exit type (or shape) can be for example a smooth contraction nozzle, a rectangular channel exit, a radially countered nozzle, or may be any exit with sharped-edge. Different shapes of rectangular nozzles are shown in Figure 2.10. Experimental investigations have shown that the exit type can strongly influence the development of the velocity flow fields of these jets (e.g. Sfier, 1979, Deo et al., 2007b).

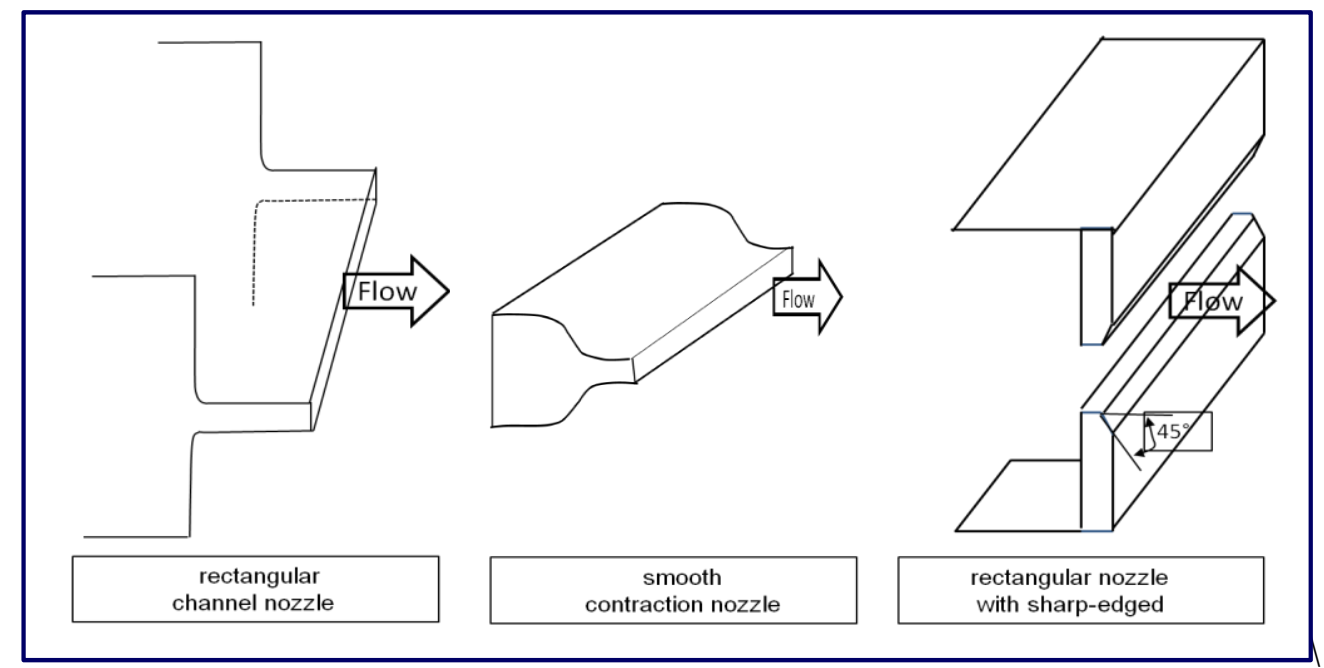

Figure 2.10: Different types of rectangular nozzles: Rectangular channel nozzle, redrawn after Krothapalli et al., (1981), and rectangular nozzle with sharp-edged, redrawn after Deo, (2005). 


\section{d) Sidewalls and/or Endplate}

Sidewalls are two parallel plates attached to the short sides of the nozzle, practically establishing a bounding wall extending the nozzle sidewalls in the downstream direction of jet propagation. They have been used in many previous studies (e.g. Bradbury, 1965, Gutmark \& Wygnanski, 1976, Deo et al., 2007a, Deo et al., 2007b, Deo et al., 2008, Suresh et al., 2008) to prevent the jet to entrain in the spanwise direction (see Figure 2.11) and hence improve the two dimensionality of the flow fields.

a)

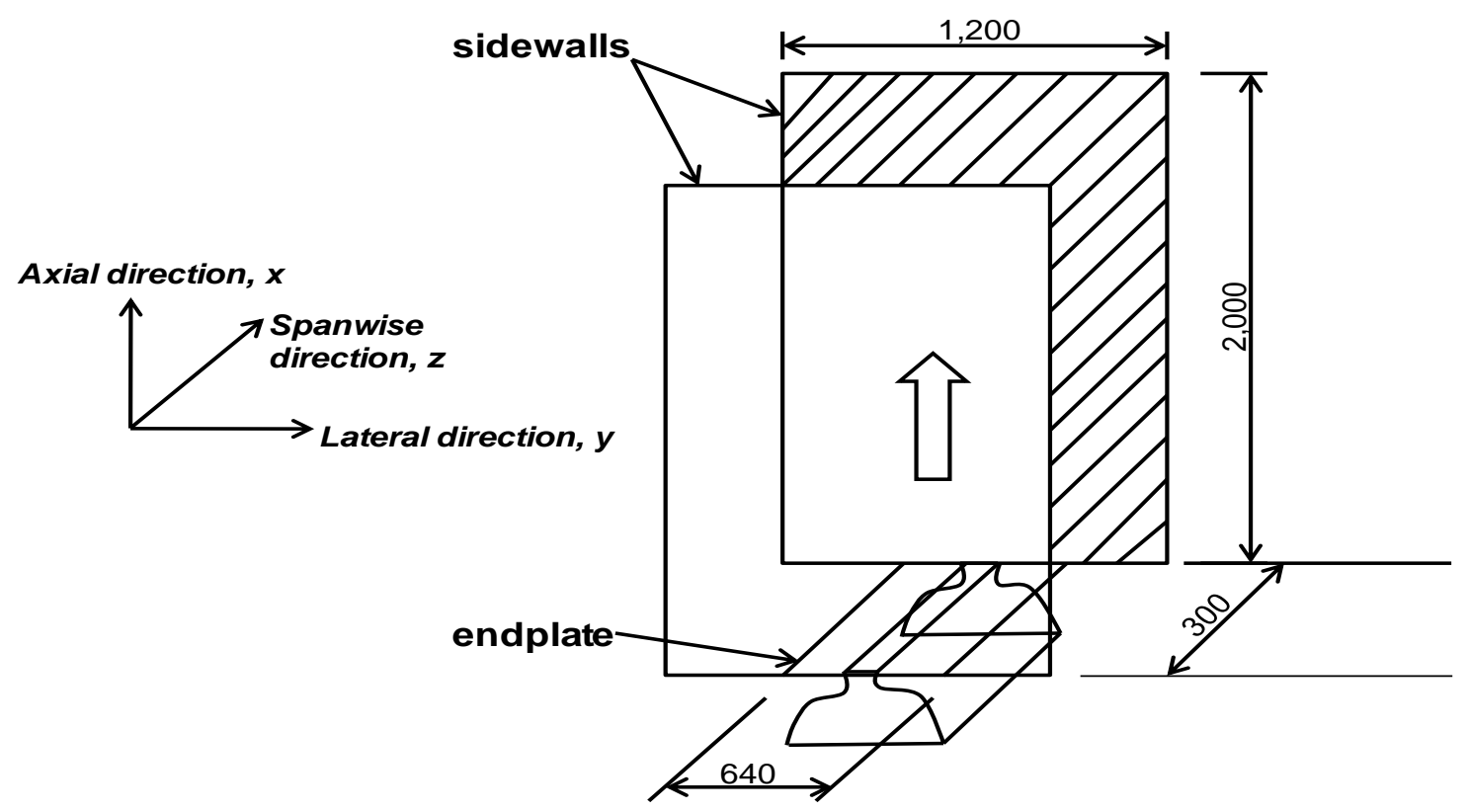

b)

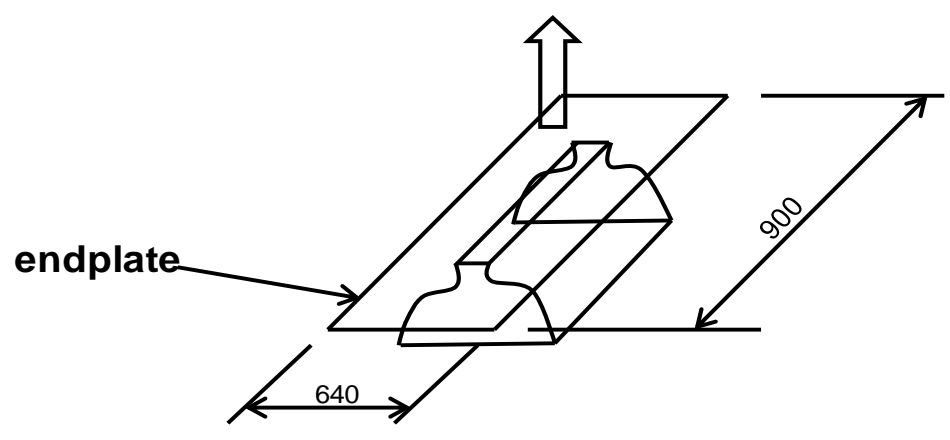

Figure 2.11: Smooth contraction nozzle: a) with sidewalls and endplate, and b) with endplate. Dimensions shown in the figure are the ones used in present investigation. (All in $\mathrm{mm}$ ). 
On the other hand endplate (or front plate) is a plate flush at the nozzle exit plane to prevent entraining from upstream of the nozzle and hence improve the twodimensionality of the flow fields. Endplate can be used either in the presence of the sidewalls as shown in Figure 2.11a (e.g. Gutmark and Wygnanski, 1976) or in the absence of the sidewalls as shown in Figure 2.11b (e.g. Quinn, 1992).

Since this thesis investigates the influence of the presence or the absence of the endplate and/or sidewalls, more information and detailed discussions will be provided in the following chapters. 


\section{Chapter 3}

\section{REVIEW}

\subsection{Literature review}

As was demonstrated in previous chapters, turbulent rectangular jets are used in many engineering fields but are also important to fundamental fluid mechanics. Thus, numerous studies have been conducted on these jets.

This thesis reports experimental investigations on the effects of the sidewalls and/or endplate on the development of turbulent rectangular jets. To understand the mechanics of a rectangular jet, one needs to better understand the mechanics of plane and round jets. Hence, a review on these jets will be addressed below.

A round jet is produced from a round as shown in Figure 2.3. Three regions of the round jet can be identified based on the velocity fields; these are the near field, the intermediate field and the far field. The near field region is also termed as the initial flow or development region. In this region the velocity is nearly similar. It is named as named the potential core. It is usually found within $0 \leq x / D \leq 6$. The far field is the fully developed (or self-similar) region where the thin shear layer approximations hold. The near and intermediate fields together comprise the developing portion of the jet (Ball \& Pollard, 2007).

The flow fields of plane jets have been studied based on the two dimensional concept that considers infinite length of the nozzle, though in many practical cases is not maintained as a finite nozzle's length is used (Seo \& Kwon, 2005). The ideal plane jet is statistically two-dimensional, in the dominant direction of the mean flow, $x$, and cross-stream coordinate, $y$, whereas statistics are expected to be independent of the spanwise coordinate (e.g. Deo, 2005).

The flow field of rectangular jets is characterized by the presence of three distinct regions (see Figure 1.1), as already discussed, it is defined by the decay of the centreline mean streamwise velocity, $U_{c}$, (Krothapalli et al., (1981), namely: (a) the 
potential core region $(x / D=0$ to $4-5)$, with almost constant $U_{c}$, which ends when the two shear layers along the short dimension of the nozzle meet, (b) the twodimensional region $(x / D=4-5$ to $25-30)$ in which the velocity decays at a rate roughly the same as that of a planar jet, and (c) the axisymmetric region, in which the velocity decays as in an axisymmetric jet, originating at $x / D=25-30$, the location where the two shear layers along the long dimension of the nozzle meet. The extent of these regions has been found to be strongly dependant on the different initial and boundary conditions such as Reynolds number e.g. Namer \& Ötügen, 1988, Deo et al., 2008, Suresh et al., 2008, aspect ratio e.g. Krothapalli et al., 1981, Deo et al., 2007a, sidewalls e.g. Hitchman et al., 1990, Deo et al., 2007c, nozzle type e.g. Deo et al., 2007b, and type of the initial boundary layer e.g. Hussain \& Clark, 1977.

The effects of Reynolds number on the development of turbulent jets issuing from rectangular nozzles have been extensively studied. Lemieux \& Oosthuizen, (1985) investigated the effects of Reynolds number on the flow characteristics of a turbulent jet issuing from a rectangular slot of aspect ratio, $A R=58$ with sidewalls. The mean velocity and turbulent stresses in the flow field were measured with hot wire anemometry at six discharge Reynolds number values in the range 700-4,200. Their results indicated a strong influence of the Reynolds number on the lateral and normal shear stresses, jet decay and spreading rates, but smaller influence on the longitudinal normal stress levels and no effects on the normalized mean velocity profiles. The results of Lemieux \& Oosthuizen, (1985) were limited to smaller range of Reynolds number. Namer \& Ötügen, (1988) extended the range of Reynolds number investigated by Lemieux \& Oosthuizen, (1985). They have studied a free plane air jets of aspect ratio, $A R=56$ at Reynolds number over the range of $1,000 \leq R e \leq 7,000$ using both $H W A$ and $L D A$ techniques. Namer \& Ötügen, (1988) have found a strong influence of Reynolds number on the flow fields of their jets. Specifically, they have observed that increasing Reynolds number has decreased the jet potential core length. This implies that, with the increase of the Reynolds number, the near field mixing has been increased. The jet spread and decay rates were found to be decreasing with the increase of Reynolds number. The effects of Reynolds numbers on the development of rectangular jet was also assessed by Chua \& Lua, (1998) for low aspect ratio, $A R=6$ jets. Two Reynolds numbers were tested (2,759 and 5,517). Their hot wire measurements showed that the rms velocity distributions established approximate 
self-preservation by $x / D=12$ for both jets. But the mean velocity distributions became self-similar at about $x / D=4$ and 6 at Reynolds number, $R e=5,517$ and 2,759 respectively. With the increase of the $R e$, a slight decrease in the spread rate was also noticed. They have reported an increase of the mean streamwise velocity values at the edges of both jets. This trend was more obvious for the jet with lower Reynolds number. They have attributed this increase in the mean streamwise velocity values to the presence of the sidewalls. This trend was also evidenced in previous investigations where sidewalls were present e.g. Daves et al., (1975) and Moum et al., (1983).

Recently, Suresh et al., (2008) investigated the transitional characteristics of plane turbulent jets using hot wire anemometry. Their measurements have been performed for a jet issuing from a rectangular nozzle of aspect ratio 20 in the presence of sidewalls, for Reynolds numbers varying in the range $250 \leq \operatorname{Re} \leq 6,250$. Their measurements revealed that, for low Reynolds number jets, the similarity of the mean velocity distribution take place at early axial distance compared the turbulence intensity profiles. It was also found that with increasing Reynolds number the jet's spreading and decay are decreasing. The turbulent field was found to attain selfpreservation at about a nondimensional axial distance of 20. Deo et al., (2008) have investigated a larger range of Reynolds number (i.e. 1,500 to 16,500) than Suresh et al., (2008). Their measurements have confirmed the dependence of these jets on Reynolds number that have been found by other investigators. Also, their results indicate that the jets have not reached their asymptotic state even at the highest Reynolds number tested i.e. $R e=16,500$. And therefore, it is may be recommended to study the influences of higher range of Reynolds number on rectangular jet flows. The present thesis will try to provide some information regarding this aspect.

Nozzle aspect ratio is an important boundary condition. It strongly influences the dimensionality of the velocity fields of jets issuing from rectangular nozzles (e.g. Mi et al., 2005). Significant information on the effects of nozzle aspect ratio on downstream development is available for turbulent jets issuing from rectangular nozzles. Available studies on the influence of aspect ratio on rectangular jets of moderate aspect ratios can provide a useful insight into plane jets of high aspect ratio and vice versa, since both jets have got a region of planar flow (e.g. Krothapalli et al., 
1981). Trendscoste \& Sforza, (1967) investigated a range of aspect ratio between 2.5 and 40. Their study found a decrease in length of the jets' potential cores, an increase in the decay of mean centerline velocity and an increase in jet spreading rate with an increase in nozzle aspect ratio. Sfeir in 1979 measured different rectangular jets using $\mathrm{x}$-wire probe to assess the influence of aspect ratio over the range $10 \leq A R \leq 60$. He obtained similar results to Trentacoste \& Sforza, (1967). Marsters \& Fotheringham, (1980) also investigated the influence of smaller aspect ratios between 3.4 and 12 at Reynolds numbers between 33,000 and 57,700, using total head tubes and hot wire anemometry. Their spanwise velocity distributions showed saddle-back behavior. This saddle-back behavior is suppressed in the smaller aspect ratio jets. Many other studies were followed focusing on the effects of aspect ratio, such as those of Krothapalli et al., (1981), Tsuchiya et al., (1985) and Quinn, (1992). Krothapalli et al., (1981) provided the mean and turbulent velocity fields using $\mathrm{x}$ - sensor hot wire anemometry over an axial distance of 115 nozzle widths. Four free jets with different aspect ratio (i.e. 5.5, 8.3, 12.5 and 16.6) were investigated in the absence of the endplates and sidewalls. They have reported a dependence on the aspect ratio of the location where the jet may be assumed to be of axisymmetric type. This location goes upstream toward the nozzle's exit with the decrease of the aspect ratio.

Quinn, (1992) also gave attention to the effect of aspect ratio on rectangular jets issuing from rectangular slots with sharp-edge (with endplate and without sidewalls). He investigated four aspect ratios (2, 5, 10 and 20). He further confirmed the dependence of the velocity flow fields of rectangular jets on their aspect ratios. Later, Lazanova \& Stankov, (1998) investigated the effect of aspect ratio on the development of rectangular jet in the range between 3 and 10. Their results also confirmed the dependence of the velocity fields of rectangular jets on aspect ratio. In 2005, Mi et al., have undertaken an investigation to characterize the turbulent jets issuing from high aspect ratio rectangular jets. Their aspect ratio range was varied between 15 and 120 in absence of endplate and sidewalls. Their measurements were conducted by a normal hot wire probe over a downstream distance of up to 160 slot width at Reynolds number of 10,000. They found that these jets can be characterized by three distinct zones: an initial quasi-plane jet zone, a transition zone and a final quasi-axisymmetric-jet zone. The extent of the quasi-plane zone was found to increase with the increase of aspect ratio while other regions decrease. Recently, Deo et al., (2007a) investigated the effects of nozzle aspect ratios between 15 and 72 on 
turbulent jet issuing from rectangular nozzle in the presence of the sidewalls and absence of endplate. Single hot wire anemometry probe was used to collect measurements up to 85 nozzle width at Reynolds number, $R e=1.80 \times 10^{4}$. Their results have further showed the dependence of the near and far flow fields of plane jet flows on the nozzle's aspect ratio.

Besides nozzle aspect ratio, experimental evidence revealed that the shape of the rectangular nozzle (or nozzle's type) can influence the jet's flow fields. Many efforts have been devoted to study the differences among different rectangular nozzle shapes. Sfier, 1979 studied two groups of rectangular nozzles namely rectangular slots and rectangular channels. He has found differences in the development of the jets produced from the two nozzle shapes. Mi et al., (2000) investigated centerline mean flow and turbulence characteristics of different jets issuing from different nozzle types (i.e. a smoothly contoured circular, an elliptical, a triangular, a square, a rectangular, a cross-shaped and a star-shaped). Their results have shown increased mixing levels of the noncircular nozzles in comparison to the round one, particularly the triangle nozzle has shown the greatest mixing level. Attention was also given to the influence of nozzle type on turbulent jets issuing from round nozzles (e.g. Mi et al., 2001, Antonia \& Zhao, 2001). Mi et al., (2001) investigated axisymmetric free jets from a contoured nozzle, an orifice plate and a pipe. They have found the jet issuing from orifice plate has the highest mixing rate with the ambient. The type of the round nozzle determines the initial streamwise velocity profile. Figure 3.1 shows the various initial velocity profiles of different types of round nozzle of Mi et al., 2001 (i.e. the smooth contraction nozzle produces a top-hat initial velocity profile, sharped-edge rectangular slot produces a saddle back initial velocity profile and pipe produces fully turbulent and developed initial velocity profiles). Antonia \& Zhao, (2001) studied two round jets. The first is issued from smoothly contracting nozzle and the other from pipe. Their measurements confirmed the difference between these two jet configurations though both jets reached the self-preservation at the same downstream location. 


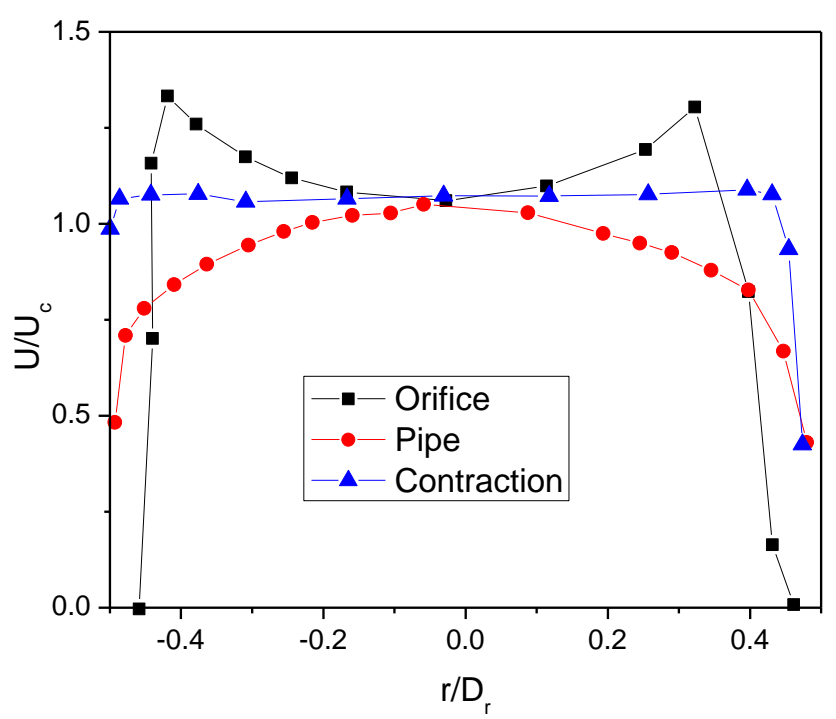

Figure 3.1: Initial velocity profiles from a sharp-edged orifice plate, a smooth contraction nozzle, and a long pipe for a round jet. Data extracted from Mi et al., (2001).

The relationship of turbulent jets and the type of initial boundary layer (e.g. laminar or turbulent) have also been given attention. Hussain \& Clark, (1977) have investigated the effect of the type of the initial boundary condition on the development of turbulent jets issuing from nozzle type and channel of 44 aspect ratio with endplate using $\mathrm{x}$-wire probe. The two laminar boundary layer jets were produced from the nozzle type at $R e=32,550$ and 81,400 . And at the same Reynolds numbers, the two turbulent boundary layer jets from the rectangular channel exit were produced. Their investigation revealed that, the laminar boundary layer lead to higher growth rate of longitudinal turbulence intensity, and the mass flux. An increase in the total average streamwise momentum was observed for both laminar and turbulent boundary condition jets, higher in the former one. Chambers et al., (1985) measured the mean and turbulent velocity and temperature fields of two plane jet (one issuing from contraction with initial top hat velocity profile and initial laminar boundary condition, and the other issuing from two dimensional duct with fully developed velocity profile) using schlieren photography and $H W A$. Their results have shown strong influence of the type of the initial boundary layer on the formation of the mixing layer structures. The mixing layer structures were strongly organized and 
symmetric about the centerline in the laminar boundary condition jet while in the other configuration, turbulent structures were three dimensional and asymmetric about the centerline.

The above discussions reveal the importance of the various initial and boundary conditions to the development of rectangular and plane jets. Boundary conditions such as sidewalls (two parallel walls confining the jet at the planes of the nozzles' short sides) and endplates (or front plates: a wall flush at the nozzle exit plane), have been used in many studies. Many investigators have used endplate (e.g. Hussain \& Clark, 1977, Quinn, 1992), sidewalls (e.g. Bradbury, 1965, Deo et al., 2007a, Suresh et al., 2008) or both together; sidewalls and endplate (e.g. Gutmark \& Wygnanski, 1976, Browne et al., 1986) to control the dimensionality of the flow fields.

As was mentioned in chapter 1, the experimental set-ups of previous studies can be classified into four groups based on the presence or absence of sidewalls and/or endplates, namely:

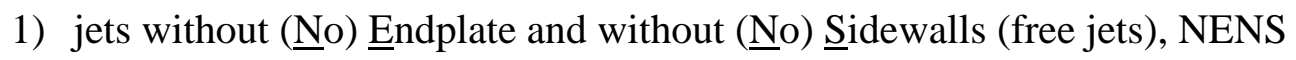
(e.g. Trentacoste \& Sforza, 1967, Krothapalli et al., 1981, Namer \& Ötügen, 1988, Papadakis \& Staiano, 1993, Lazanova \& Stankov, 1998, Mi et al., 2005, Cavo et al., 2007, Deo et al., 2007c).

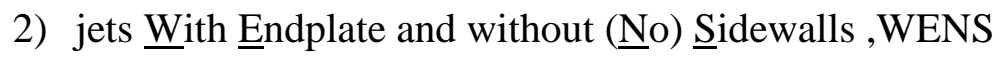
(e.g. Sato, 1959, Sforza et al., 1965, Hussain \& Clark, 1977, Masrsters, 1980, Hitchman et al., 1990, Quinn, 1992, Papadakis \& Staiano, 1993).

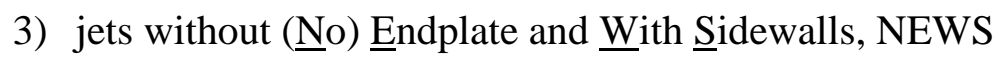

(e.g. Bradbury, 1965, Everitt \& Robins, 1978, Lemieux \& Oosthuizen, (1985), Chua \& Lua, 1998, Deo et al., 2007a, Deo et al., 2007b, Deo et al., 2007c, Deo et al., 2008, Suresh et al., 2008).

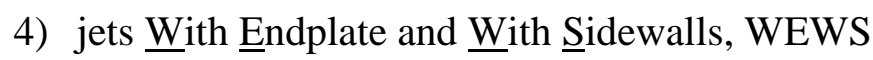

(e.g. Gutmark \& Wygnanski, 1976, Antonia et al., 1983, Hitchman et al., 1990). 
As evidenced from the above classification, sidewalls and/or endplate have been used in many investigations at various initial conditions (e.g. different aspect ratio and Reynolds numbers, nozzle types), but few experiments have been devoted to study their effects on turbulent jets issuing from rectangular nozzles (Hitchman et al., 1990, Papadakis \& Staiano, 1993 and Deo et al., 2007c).

Hitchman et al., (1990) have experimentally investigated the effects of sidewalls on development of turbulent jet issuing from a rectangular nozzle of aspect ratio, $A R=60$ and Reynolds number, $R e=7,230$ in the presence of an endplate. Their measurements indicate that the decay rate is slower, but the spread rate is higher without sidewalls. This is an interesting result since intuitively one would expect a jet, which spreads faster to also decay faster. These investigators also found that although the kinematic momentum flux is nearly conserved in the jet without sidewalls, it is decreasing significantly in the presence of the sidewalls. The implementation of the single hot wire probe and the absence of any measurements either on the lateral or spanwise directions have limited the amount of information provided. Measuring more than one velocity component provides estimates of higher order statistics and correlation which are essential for the assessment of the sidewalls effects. After almost two decades, Deo and his team (Deo et al., 2007c) have again explored the effects of the sidewalls on turbulent jets issuing from rectangular exit. They have selected the same aspect ratio that Hitchman et al., (1990) have used (i.e. $A R=60)$ and nearly similar Reynolds number, $R e=7,000$ to compare two plane jets (without endplate), one with sidewalls and the other without sidewalls. Based on their single probe hot wire measurements, it was found that shorter potential core lengths ( $4 \mathrm{D}$ for jet without sidewalls, $6 \mathrm{D}$ for the jet with sidewalls), higher spread rate ( 0.14 for the jet without sidewalls, 0.11 for the jet with sidewalls) and higher decay rate (0.2 for the jet without sidewalls, 0.17 for the jet with sidewalls) are associated with the jet without sidewalls. They have also observed a longer two dimensional flow region in the presence of the sidewalls. While in the absence of the sidewalls, their jet behaved statistically like axisymmetric jet at about 60 nozzle width.

It is worth noting that both of the above investigations have focused on the same aspect ratio (i.e. $A R=60$ ) and similar Reynolds number $\sim 7,000$. For this large aspect 
ratio, the jet centreline velocity is expected to have planar jet behaviour up to a considerable downstream distance from the exit nozzle, whereas for rectangular jets of smaller aspect ratio a weaker planar jet behaviour may be anticipated (Mi et al., 2005). In other words, the downstream location where the centreline velocity first assumes axisymmetric characteristics is expected to move upstream, toward the exit of the jet, with the decrease of the aspect ratio (Krothapalli et al., 1981).Therefore, there is a need to explore the influence of the sidewalls on the development and mixing, in the near field (i.e. potential core) and in the two-dimensional region (i.e. self persevering), for jets issuing from rectangular nozzles of moderate and smaller aspect ratio. Our investigation will try to provide information on this need.

Studying the influence of an endplate, Papadakis \& Staiano, (1993) measured the flow field of two jets, one with endplate and the other without, issuing from a rectangular nozzle of aspect ratio, $A R=40$, at Reynolds number, $R e=9,800$. Based on $L D A$ measurements, they have found that both jets produce very similar velocity profiles indicating that the endplate did not have significant effect on the jet velocity distribution. On the other hand Abdel-Rahman et al., (1997) investigating the use of an endplate at the exit plane of an axisymmetric round jet have found that, introducing an endplate at the nozzle exit caused a reduction in the velocity spreading rate and reduced kinematic mass flow rate. The reduction in the kinematic mass flow rate of Abdel-Rahman et al., (1997) investigation is in agreement with Kotsovinos, (1978) and Schneider, (1985) studies. Kotsovinos, (1978) reformulated the equation of the momentum flow in a turbulent jet and used it to show that the conservation of momentum is an inaccurate approximation for a plane jet with an endplate. Furthermore Schneider, (1985) has shown that in a turbulent jet (round or plane) with endplate the axial velocity decreases more rapidly and the jet suffers momentum reduction in the streamwise direction due to the induced outer flow, concluding that the momentum is conserved only for the case of a jet without endplate. It should be noted that, the investigation of Papadakis \& Staiano, (1993) was limited since most of the reported profiles were focused on the near mean field of only (i.e. $x / D \leq 18$ ). Furthermore, the findings of Papadakis \& Staiano, (1993) were based on experiments conducted in the absence of sidewalls. Since sidewalls were found to strongly affect the development of rectangular/plane jets (e.g. Deo et al., 2007c), it will be interesting 
to study the influence of endplates on jets with sidewalls beside jet without sidewalls. Information on the influence of the endplate in the presence and absence of sidewalls will be provided in this thesis.

In summary, the above review reveals that there is a shortage of information on the influence of the 1) endplate, 2) sidewalls, and 3) both endplate and sidewalls on the development of turbulent rectangular jets although these flows are under investigation since many decades. Therefore, the present investigation will try to provide information for these needs by investigating a rectangular jet of aspect ratio, $A R=15$ at three Reynolds number, $R e=10,000,20,000$ and 30,000 using x-wire probe $H W A$. 


\section{Chapter 4}

\section{EXPERIMENTATION}

\subsection{Experimental set-up}

The experimental set-up used in our investigation (Figure 4.1) is the same one used in previous investigation of Cavo et al., (2007). It is located at the Laboratory of Thermodynamics in the campus of University of Patras, Patras, Greece.

The experimental set-up is mounted vertically and consists of smooth contraction exit, settling chamber, and blower. The set-up facilitated the implementation of endplate and/or sidewalls when required. The experimental arrangement was acoustically isolated from external driven noise. In the coming sections, a description of each part of the experimental set-up will be given below.

\subsubsection{Nozzle exit}

As was discussed in the previous sections (chapter 2 and 3), nozzle exit type influences the jet's flow either in the near or far fields (e.g. Deo et al., 2007b). The nozzle used in this investigation is a smooth contraction rectangular nozzle. The length of the long and short sides of the nozzle are $L=300 \mathrm{~mm}$ and $D=20 \mathrm{~mm}$ respectively, with aspect ratio, $A R=L / D=15$. The present nozzle, like conventional smoothly-contracting ones, produces an approximately "top-hat" mean velocity profile at the exit.

\subsubsection{Settling chamber}

Before reaching the nozzle, the air is passed through a settling chamber, which contains five screens ( $1 \mathrm{~mm}$ square mesh, $0.3 \mathrm{~mm}$ diameter wire) at $5 \mathrm{~cm}$ spacing to reduce streamwise turbulence intensity. A honey comb is installed at the entrance of settling chamber to assist the smoothing and alignment of the flow breaking large scale eddies and reducing cross plane turbulence intensity components. 

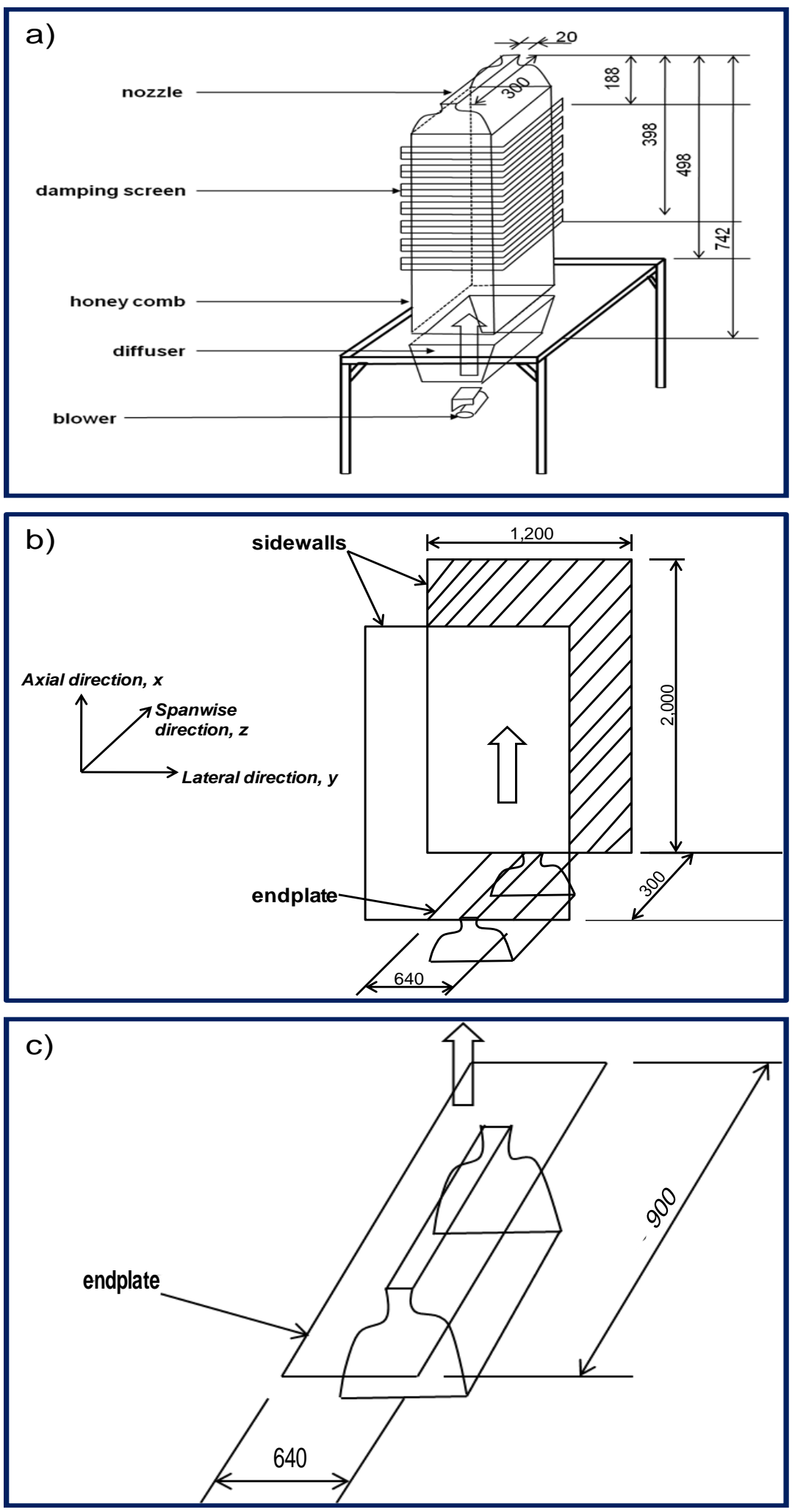

Figure 4.1: Experimental apparatus, a) jet facility, and b) rectangular nozzle with endplate and sidewalls, and c) rectangular nozzle with endplate. (all dimensions in $\mathrm{mm})$. 


\subsubsection{Blower}

A centrifugal blower air supply system of $1.5 \mathrm{~kW}$ power was used to provide the airflow to the diffuser, which decelerates the flow and converts the dynamic pressure to static in order to minimize energy losses and preserve the uniformity of the flow field.

\subsubsection{Endplates and sidewalls}

The set up facilitated the implementation of sidewalls and/or endplates when required. Two parallel plates made of Perspex of $5 \mathrm{~mm}$ thickness, 2,000 $\mathrm{mm}$ in length and 1,200 $\mathrm{mm}$ in width were used as sidewalls (see Figure.4.1-b). They were attached to the slot's short sides extending in the axial direction, supported by a suitable frame to minimize vibrations during operation. Another Perspex plate of $5 \mathrm{~mm}$ thickness, $900 \mathrm{~mm}$ length and $640 \mathrm{~mm}$ width was used as an endplate flush at the nozzle's exit plane. In table 4.1 the dimensions of the sidewalls and endplate in relation to the characteristics of the jet development, indicating the degree of attenuation of intended boundary conditions, are presented for the present study and related experiments.

Table 4.1 Dimensions of sidewalls and endplates in relation to characteristic dimensions of jet development

\begin{tabular}{|c|c|c|c|c|c|c|c|c|c|c|c|}
\hline Source & $\begin{array}{l}\text { Jet } \\
\text { type }\end{array}$ & $\begin{array}{c}\text { Nozzle } \\
\text { dimensions } \\
(\mathrm{mm}) \\
\end{array}$ & $\begin{array}{c}x_{\max } \\
(\mathrm{mm})\end{array}$ & $\begin{array}{c}y_{c}\left(x_{\max }\right) \\
(\mathrm{mm})\end{array}$ & $\begin{array}{c}L_{s w} \\
(\mathrm{~mm})\end{array}$ & $\begin{array}{l}W_{s w} \\
(\mathrm{~mm})\end{array}$ & $\begin{array}{l}W_{e p} \\
(\mathrm{~mm})\end{array}$ & $\frac{L_{s w}}{x_{\max }}$ & $\frac{W_{s w}}{y_{c}\left(x_{\max }\right)}$ & $\frac{W_{e p}}{H}$ & $\frac{W_{e p}}{y_{c}\left(x_{\max }\right)}$ \\
\hline $\begin{array}{l}\text { Quinn, } \\
\text { (1992) }\end{array}$ & WENS & $17.96 \times 89.8$ & $\begin{array}{c}1359 \\
(75 \mathrm{H})\end{array}$ & 134 & & & 2440 & & & 136 & 18.2 \\
\hline $\begin{array}{l}\text { Deo et al., } \\
(2007 \mathrm{c})\end{array}$ & NEWS & $5.6 \times 340$ & $\begin{array}{c}560 \\
(100 \mathrm{H})\end{array}$ & 64 & 2000 & 1800 & & 3.6 & 28 & & \\
\hline $\begin{array}{l}\text { Gutmark \& } \\
\text { Wygnanski } \\
\text { (1976) }\end{array}$ & WEWS & $13 \times 500$ & $\begin{array}{c}1560 \\
(120 \mathrm{H})\end{array}$ & 143 & 2000 & 2000 & 2013 & 1.28 & 13.93 & 155 & 14 \\
\hline $\begin{array}{l}\text { Hitheman } \\
\text { et al., } \\
\text { (1990) }\end{array}$ & WEWS & $10 \times 600$ & $\begin{array}{c}748 \\
(75 \mathrm{H})\end{array}$ & 78 & 1850 & 1100 & 2010 & 2.47 & 14.1 & 201 & 25.7 \\
\hline $\begin{array}{l}\text { Antonia et } \\
\text { al., (1983) }\end{array}$ & WEWS & $12.7 \times 254$ & $\begin{array}{c}889 \\
(70 \mathrm{H})\end{array}$ & 99 & 1500 & 700 & 254 & 1.69 & 7.07 & 20 & 2.56 \\
\hline $\begin{array}{l}\text { Present } \\
\text { work }\end{array}$ & NEWS & $20 \times 300$ & $\begin{array}{c}700 \\
(35 \mathrm{H})\end{array}$ & 76 & 2000 & 1200 & 640 & 2.85 & 15.8 & 32 & 8 \\
\hline
\end{tabular}




\subsection{Hot wire anemometry}

Anemometers are devices that are used to measure the velocity of fluids. This is achieved by using thin tungsten/platinum wire or thin metallic film which is heated to a temperature higher than the average temperature of the fluid. Since the temperature of the probe will be cooled by the fluid flow, the electronic circuits of the anemometer will stabilize and maintain it. This will make the anemometer to be very sensitive to the rate of cooling by the fluid. As the fluid velocity increases the rate of cooling also increases. Then this velocity is translated to varying voltage which has a nonlinear relationship with the velocity of fluid. The voltage signal will undergo signal conditioning to eliminate noise and improve the signal/noise rate. And after a prober calibration of the probe channels, fluid velocity with an accuracy of $0.005 \%$ or more can be measured. This depends on the measurement range and calibration's quality. Two types of hot wire anemometry systems can be found 1) Constant Temperature Hot-Wire Anemometry, (CTA), and 2) Constant Current Hot-Wire anemometry, (CCA) (AN-1003, Hot wire \& film anemometry systems, User's Manual).

\subsubsection{Constant Temperature Hot-Wire Anemometry (CTA)}

CTA has been used in the present investigation. The main parts of constant temperature hot wire anemometry, are shown in Figure 4.2.

a)

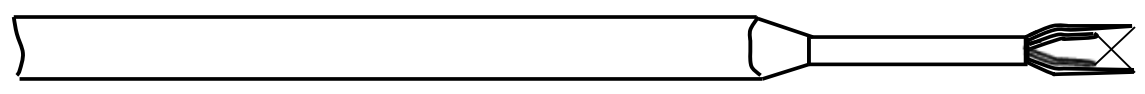

b)

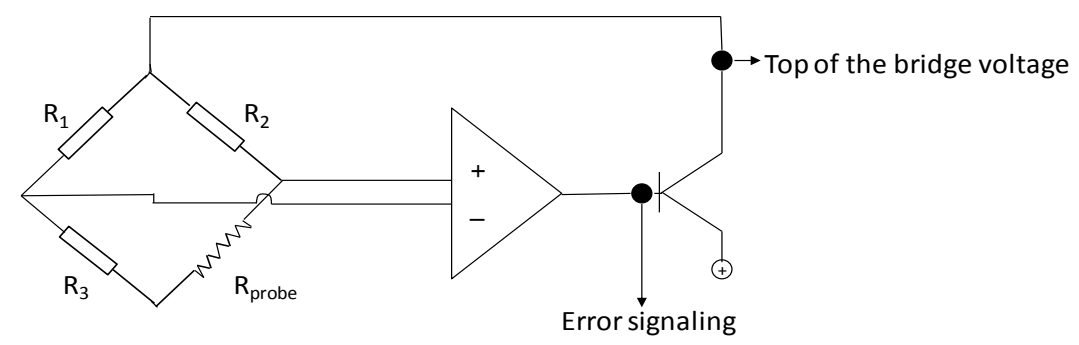

Figure 4.2: Hot wire anemometry components a) typical $\mathrm{x}$-wire probe, and $\mathrm{b}$ ) the Wheatstone bridge. Redrawn after AN-1003, Hot wire \& film anemometry systems, User's Manual. 
And they are i) the probe, ii) electronic feedback circuit which drives and produces a voltage proportional to velocity, and iii) signal conditioning circuit which provides noise reduction and amplification (AN-1003, Hot wire \& film anemometry systems, User's Manual).

\subsubsection{Working principle}

When the probe is connected to the Wheatstone bridge which is balanced at room temperature, the feedback circuit is continuously trying to balance the bridge. The probe resistance will decrease due to the cooling by convection resulting in increase of the voltage which is measured across the horizontal diagonal of the bridge. This voltage will be fed into servo amplifier causing an increase of probe's temperature and its resistance to the point where the bridge is balanced again the voltage across the horizontal diagonal of the bridge becomes zero. If the fluid flow is reduced, the same previous sequences occur except that the currents are inverted. The output voltage of the servo amplifier indicate to the degree to which the probe is heated, will give us a measure of the rate of cooling of the probe of the fluid (AN-1003, Hot wire $\&$ film anemometry systems, User's Manual).

The sensitivity of the circuit to changes of probe resistance and adjust the heating or the frequency response of the circuit depends on three variables 1) overheat ratio (OHR), 2) loop gain, and 3) cable compensation. The OHR is the ratio of the electrical resistance of the heated wire to the electrical resistance of unheated wire at reference atmospheric condition. It is usually set by the operator to fix the temperature difference between the probe and ambient air. Higher OHR will result in greater system bandwidth and higher sensitivity of the probe when the fluid flow is changing. The probe may burn at some high temperature hence it is advised to set OHR that is recommended by the probe's manufacturer. The probe also can be burnt due to improper damping of the anemometer circuit that produces oscillations. (AN1003, Hot wire \& film anemometry systems, User's Manual)

The input of the signal conditioning unit is the voltage across the bridge. While the output can be used and connected to an oscilloscope to give immediate observation of the fluid velocity. Meanwhile it is recorded by a computer by using data acquisition 
system, data storage devices or analog to digital converter. The conversation of output voltages to fluid velocities needs building a calibration graph since the relationship between the velocity and voltage is not linear. This relationship can be plotted using $5^{\text {th }}$ degree polynomial. The calibration must be performed for every working channel. If the voltage signal is acquired by an $\mathrm{A} / \mathrm{D}$ converter, extra linear conversion must be performed (AN-1003, Hot wire \& film anemometry systems, User's Manual).

Conditioning of the signal which is produced by the fluctuating voltage on the probe must be done to have useful measurements. The main functions of signal conditioning are to amplify, offset and filter the signal. This can be done by the experimenter (AN1003, Hot wire \& film anemometry systems, User's Manual). A signal before and after conditioning is shown in Figure 4.3.

a)

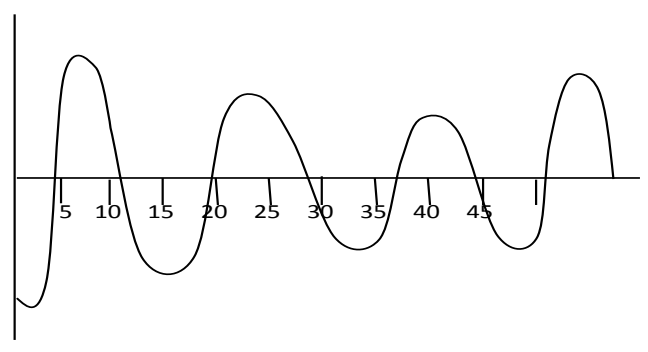

b)

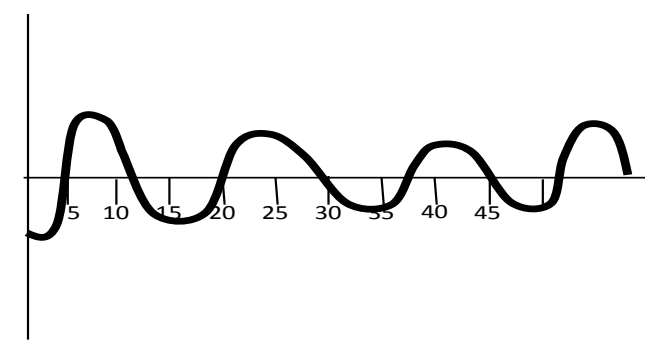

Figure 4.3: Signal a) before conditioning, and b) after conditioning. Redrawn after AN-1003, Hot wire \& film anemometry systems, User's Manual.

The working principle of Constant Current Anemometry (CCA) is very similar to CTA. As was explained earlier, a constant resistance is maintained in the probe leg of the bridge to be able to measure the velocity. But in CCA, a constant current is passed through the probe leg to be able to measure the temperature of the fluid flowing over the probe (AN-1003, Hot wire \& film anemometry systems, User's Manual).

\subsubsection{Noise}

To obtain stable signal, experimenter must avoid any noise which can strongly influence that signal. Among the noises these are due to the dynamic stresses caused by improper welding or soldering of the sensors; poor electrical connection (e.g. dirty 
or faulty BNC connectors) can add much noise due to the sudden voltage fluctuation; if the wires are not correctly placed, electromagnetic inference is may produced from external sources; cross talking between the channels due to ground loops; amplifier circuit will add noise due to heating; poor calibration of A/D conversion units. Noises can be introduced from other surrounding systems e.g. computers, motors etc (AN1003, Hot wire \& film anemometry systems, User's Manual).

\subsubsection{Advantages of hot wire anemometry technique}

The advantages of the hot wire anemometry technique are summarized below (Goldstein, 1996):

1) Using hot wire anemometry technique, measurements to several hundred kilohertz are easily obtained.

2) Hot wire anemometry sensors are small compared to laser velocimeters.

3) Hot wire can resolve one, two or all three components of flow by using one, two or more sensors.

4) Hot wire has good resolution since it has very low noise level.

5) Low cost of hot wire anemometry systems compared to other techniques.

6) Hot wire anemometry technique is generally easy to use however repairing the probe is complicated.

\subsubsection{Disadvantages of hot wire anemometry technique}

The disadvantages of the hot wire anemometry technique are summarized below (Goldstein, 1996):

1) At low velocity free convection effects influence the hot wire readings.

2) Hot wire technique has limitation in measuring flows with reversals.

3) Hot wire sensors are delegate, easily fragile and their repair is complicated.

\subsubsection{Selection of hot wire sensors}

Materials such are tungsten, platinum, and platinum-iridium (80\% Pt, 20\% Ir) are used in hot wire probes due to their properties and their availability in small diameters. Hot wires should have high resistivity and tensile strength and low 
conductivity. The high temperature coefficient of resistance and high strength of tungsten wires made them to be more preferred though its oxidization rate at a temperature above about $300{ }^{\circ} \mathrm{C}$ is a major disadvantage. Tungsten wire are available commercially in small diameters e.g. 2.5 micron. Platinum has a good temperature coefficient, and it doesn't oxidize. It is available in a very small diameter $(0.5$ micron). Its weakness at high temperature prevented to be the ideal material. Platinum-iridium wires do not oxides, stronger than platinum and has a low temperature coefficient of resistance. This compound is used where the wire temperature is too high (Goldstein, 1996).

\subsubsection{Calibration of hot wire anemometer}

A calibration curve from a hot wire anemometer is shown in Figure 4.4. As one can see, the output voltage is not linear with the velocity. Once the experimenter obtained similar curve that is shown in Figure 4.4, good measurements in unknown environment can be directly obtained. This will provide the fluid temperature, composition, and density in the unknown environment similar to ones during calibration, if not, they will be corrected; the turbulent intensity below $20 \%$ and the flow is incompressible. With these conditions, a hot wire probe to measure mean velocity, turbulence intensity, turbulence spectrum, waveform, and flow transients. The mean flow, true waveform, and flow transients interpreted by converting voltages to velocity from the calibration curve. Whereas, spectrum, turbulence are interpreted by measuring the slope of the curve at the operating point.

Figure 4.4: Typical calibration curve of a hot wire anemometer. Redrawn after Goldstein, (1996).

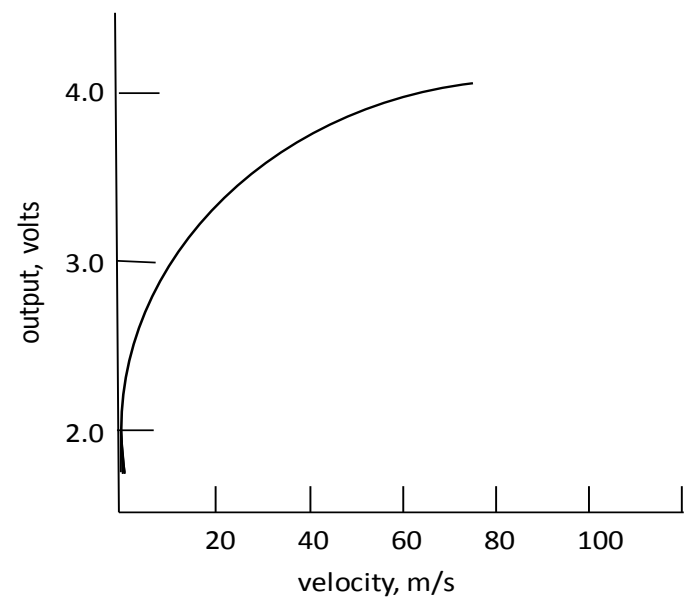


In this investigation, the $\mathrm{x}$-wire was calibrated at nozzle exit in comparison with pitot tube measurements, using on average, 10 calibration points.

\subsubsection{Measuring velocity components}

Measuring the velocity components depends on the type of flow and the type of information which is required e.g. single hot wire probe provide information about the axial velocity component, two hot wire probes provide information about two velocity components and three or more hot wires probes can provide information about all velocity field (3-components). Other complicated designed multi sensors can provide information about the velocity-vorticity fields (e.g. Cavo et al., 2007, Romeos et al., 2009).

In the present investigation, $\mathrm{x}$-probe was used to measure the two velocity components $\left(V_{1}\right.$, and $\left.V_{2}\right)$. In this case, two sensors as shown in Figure 4.5 are made sensitive to $V_{2}$ by orienting them to the mean flow. The two sensors are oriented at $90^{\circ}$ degree to each other (Goldstein, 1996).

We have for each sensor,

$$
\begin{aligned}
& V_{\mathrm{A}, \mathrm{eff}}^{2}=\left(V_{1} \cos \alpha_{1}-V_{2} \sin \alpha_{1}-V_{2} \sin \alpha_{1}\right)^{2}+\mathrm{K}_{\mathrm{T}}^{2}\left(V_{1} \sin \alpha_{1}+V_{2} \cos \alpha_{1}\right)^{2}+\mathrm{K}_{\mathrm{N}}^{2} V_{3}^{2} \\
& V_{\mathbf{B}, \mathrm{eff}}^{2}=\left(V_{1} \cos \alpha_{1}-V_{2} \cos \alpha_{1}-V_{2} \sin \alpha_{1}\right)^{2}+\mathrm{K}_{\mathrm{T}}^{2}\left(V_{1} \cos \alpha_{1}+V_{2} \sin \alpha_{1}\right)^{2}+\mathrm{K}_{\mathrm{N}}^{2} V_{3}^{2}
\end{aligned}
$$

where $\alpha_{1}$ is the angle between $V_{1}$ and sensor B.

The coordinates are usually selected such that $\overline{V_{3}}=0$, if the sensors are sufficiently long that $\mathrm{K}_{\mathrm{T}} \rightarrow 0$ and $\mathrm{K}_{\mathrm{N}} \rightarrow 1$, the Equations 4.1 and 4.2 will reduce to

$$
\begin{aligned}
& V_{\mathrm{A}, \mathrm{eff}}^{2}=\left(V_{1} \cos \alpha_{1}-V_{2} \sin \alpha_{1}\right)^{2}+v_{3}^{2} \\
& V_{\mathrm{B}, \mathrm{eff}}^{2}=\left(V_{1} \sin \alpha_{1}-V_{2} \cos \alpha_{1}\right)^{2}+v_{3}^{2}
\end{aligned}
$$

In large turbulence intensities, equations 4.3 and 4.4 cannot be further reduced, however if $\mathrm{v}_{3}$ is small then the last term can be ignored, rearranging equations 4.3 and 4.4 give: 


$$
\begin{aligned}
& V_{1}=2^{-1 / 2}\left(V_{\mathrm{A}, \text { eff }}+V_{\mathrm{B}, \text { eff }}\right) \\
& V_{2}=2^{-1 / 2}\left(V_{\mathrm{A}, \text { eff }}-V_{\mathrm{B}, \text { eff }}\right)
\end{aligned}
$$

Assuming the linearized output voltages of the two constant temperature anemometers gives $V_{1}$, and differentiating them gives $V_{2}$

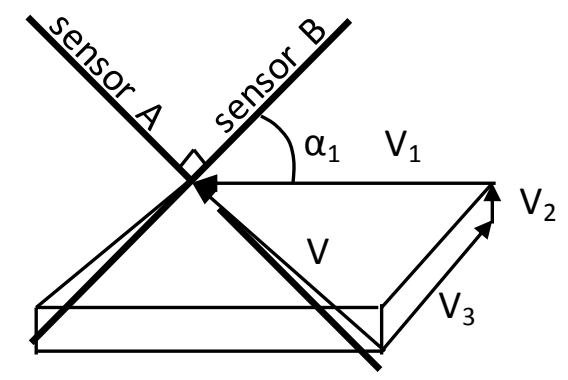

Figure 4.5: X-wire configuration. Redrawn after Goldstein, (1996).

\subsubsection{Specifications of the hot wire anemometry system and experimental conditions of the present investigation}

\section{$\underline{\text { Measuring system }}$}

1) The anemometer used in this investigation was AN-1003 model from A.A. Lab System LTD.

2) X-wire probe from Dantec Dynamics (5 micron in diameter and $1 \mathrm{~mm}$ in length).

3) Monitoring the signal was done on a GoldStar oscilloscope (OS 9020G20MHz).

4) The overheat ratio (OHR) was 1.4.

5) The sampling frequency was $2 \mathrm{kHz}$ for a time series of 16,000 samples.

6) A PCL - 814 data acquisition board attached to a personal computer was used. (100 k Hz, 16 channels A/D converter with 14-bit resolution). 


\section{$\underline{\text { Experimental conditions }}$}

1) The ambient condition was isothermal at about $30^{\circ} \mathrm{C}$.

2) Smooth contraction nozzle was used.

3) The aspect ratio of the nozzle was $A R=L / D=15$ (where $L=30 \mathrm{~cm}$ and $D=2 \mathrm{~cm}$, are the lengths of the long and small sides of the nozzle).

4) Three Reynolds numbers were tested i.e. $R e=10,000,20,000$ and 30,000 equivalent to exit velocities, $U_{0}=8,15$ and $24 \mathrm{~m} / \mathrm{s}$ respectively.

5) The ambient condition was isothermal at about $30^{\circ} \mathrm{C}$.

\subsubsection{Experimental uncertainties}

The experimental uncertainties in these experiments can be due to wire calibrations, data reduction and standard errors from measuring instrumentation. Much care was taken to reduce all uncertainties due to above mentioned sources. Calibration was sometimes repeated during experiments to avoid drifts. BNCs were checked time to time to avoid adding noises to the signals. Errors estimated by means of reverse computation of calibration data were of the order of $\pm 1.5 \%$ of $U$ for mean velocities on the centerline, resulting in uncertainties of $\pm 3 \%$ and $\pm 5 \%$ for the second and thirdorder velocity fluctuation statistics (Browne et al., 1984).

\subsection{Experimental procedure}

In order to allow a direct comparison of the results, all four configurations identified previously (using the introduced abbreviations: NENS, WENS, NEWS and WEWS) have been studied using the same basic nozzle configuration, i.e. a smoothly contracting nozzle of $A R=L / D=15$.

Streamwise and lateral velocity component measurements were conducted using $\mathrm{x}$ wire probe $H W A$, providing information about the mean and statistical properties of the flow. The measurements collected were as follow:

\section{a) Centreline}

1) Measurements were collected at $R e_{D}=10,000,20,000$ and 30,000 up to $x / D=35$, for all configurations (i.e. NENS, WENS, NEWS, and WEWS). 
b) Lateral direction (across the jet)

1) At $\operatorname{Re}_{D}=20,000$, measurements were collected at 13 different locations (i.e. $x / D=0,1,2,2.5,3,4,5,10,15,20,25,30$ and 35) for all configurations (i.e. NENS, WENS, NEWS, and WEWS). 


\section{Chapter 5}

\section{EFFECT OF ENDPLATE AND/OR SIDEWALLS ON RECTANGULAR JETS}

\subsection{Results and discussion}

\subsubsection{Mean Velocity Field}

\subsubsection{Longitudinal mean velocity}

a) Transverse distributions of longitudinal mean velocities

Mean streamwise velocity, $U$, profiles measured along the transverse direction on the central $x y$ plane are presented in Figure 5.1, for $R e=20,000$. Measurements for the four configurations (NENS, WENS, NEWS and WEWS) are depicted at downstream locations $x / D=0,1,2,2.5,3,4,5,10,15,20,25,30$ and 35 in Figures 5.1a, b, c, d, e, $\mathrm{f}, \mathrm{g}, \mathrm{h}, \mathrm{j}, \mathrm{k}, \mathrm{l}, \mathrm{m}$ and $\mathrm{n}$ respectively. Profiles are plotted as $U / U_{c}\left(U_{c}\right.$ is the streamwise mean velocity at the centreline) against $y / y_{c}\left(y_{c}\right.$ is the distance where the streamwise velocity is half of the centreline velocity at the same $x / D$ location).

Near the exit all configurations, in line with previous investigations comprising smoothly contracting nozzles, produce uniform $\left(U / U_{c} \approx 1\right)$ "top-hat" mean velocity profiles. Downstream all test cases exhibit similar trends and the measurements almost collapse for most part of the distributions $\left(y / y_{c}<2\right)$. The major differences appear at the edges of the jets where the profiles of the jets with sidewalls (NEWS, WEWS) attain systematically larger values, except at the furthest $x / D$ location. A similar behaviour of non zero mean streamwise velocities for $y / y_{c} \geq 2$ has been reported (Chua \& Lua, 1998) and observed (e.g. Daves et al., 1975, Moum et al., 1983) in investigations where sidewalls were used. Chua \& Lua, (1998) investigating rectangular jets of aspect ratio 6 , issuing with velocities $10 \mathrm{~m} / \mathrm{s}$ and $20 \mathrm{~m} / \mathrm{s}$ into a closed cavity with an outlet, characterized this phenomenon as an "upward trend" and attributed it to the presence of the sidewalls that cause a cavity effect resulting in accelerating or reducing the deceleration of the jet. The larger streamwise velocities 
at the edges of the jets NEWS and WEWS at distances from the nozzle in the range $x / D=3-30$ indicate that the presence of sidewalls leads to this increase.

Downstream, focusing on the effect of the endplates on the free jet, comparing the cases with no sidewalls (NENS and WENS), the two jets exhibit similar trends and the measurements collapse for all part of the distributions, indicating an insignificant effect of the endplate. These findings agree with those of Papadakis \& Staiano, (1993) who carried out LDA measurements in a free rectangular jet of aspect ratio $A R=40$ with and without endplate. They also found an insignificant effect of the endplate on the transverse mean streamwise velocity distributions. Similar remarks hold for the two configurations comprising sidewalls. Again no difference due to the presence of the endplate can be identified (NENS, WENS). It can therefore be concluded that the dominant effect on the streamwise velocity profile is due to the presence or absence of sidewalls whereas the presence of the endplate has an insignificant effect.

a)

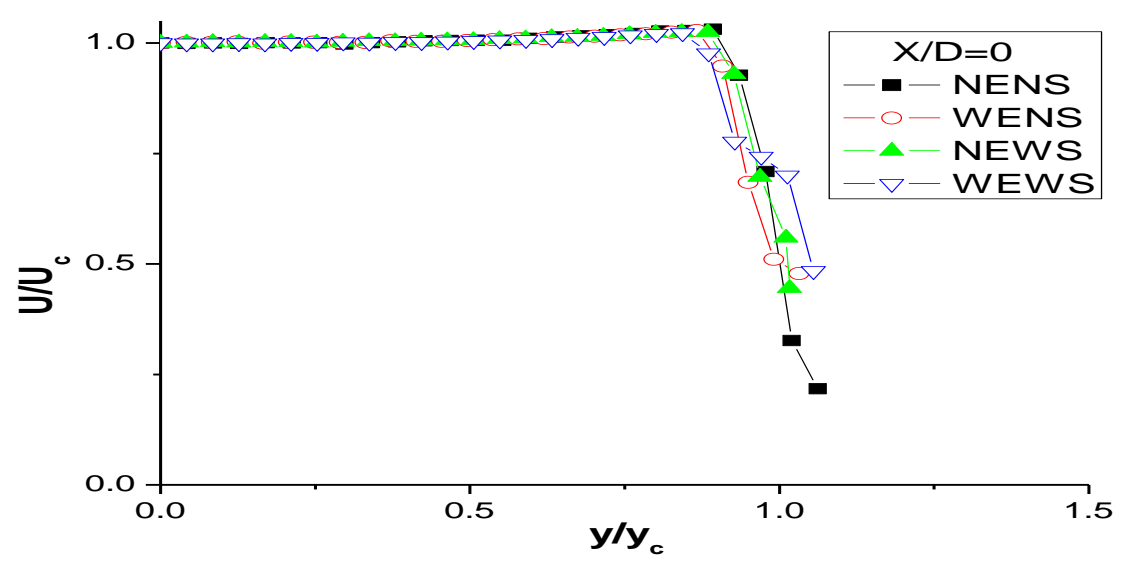

b)

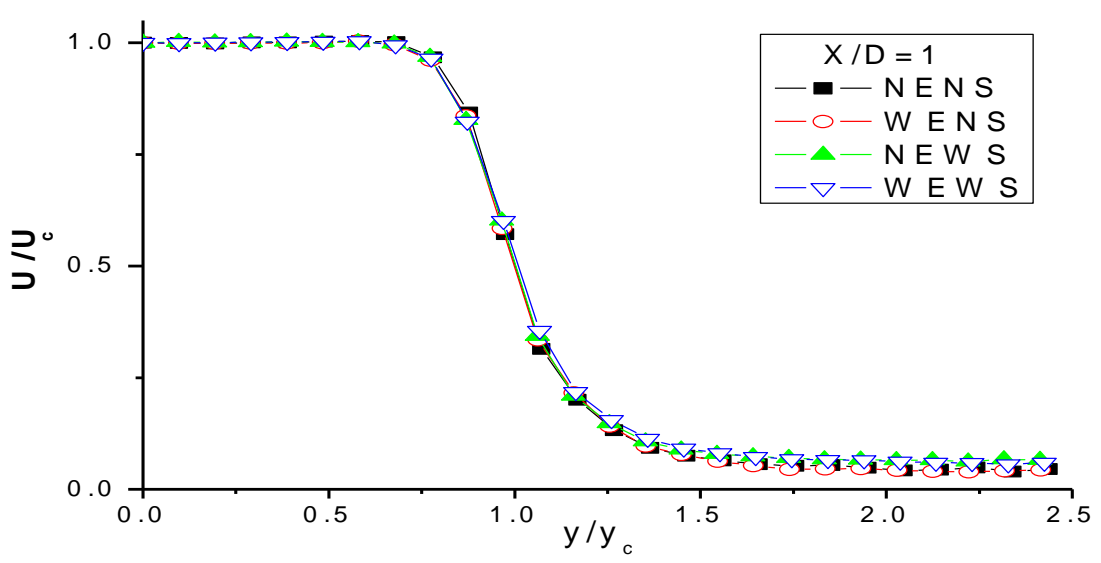

Figure 5.1: Mean streamwise velocity profiles on the central $x y$ plane. 
c)

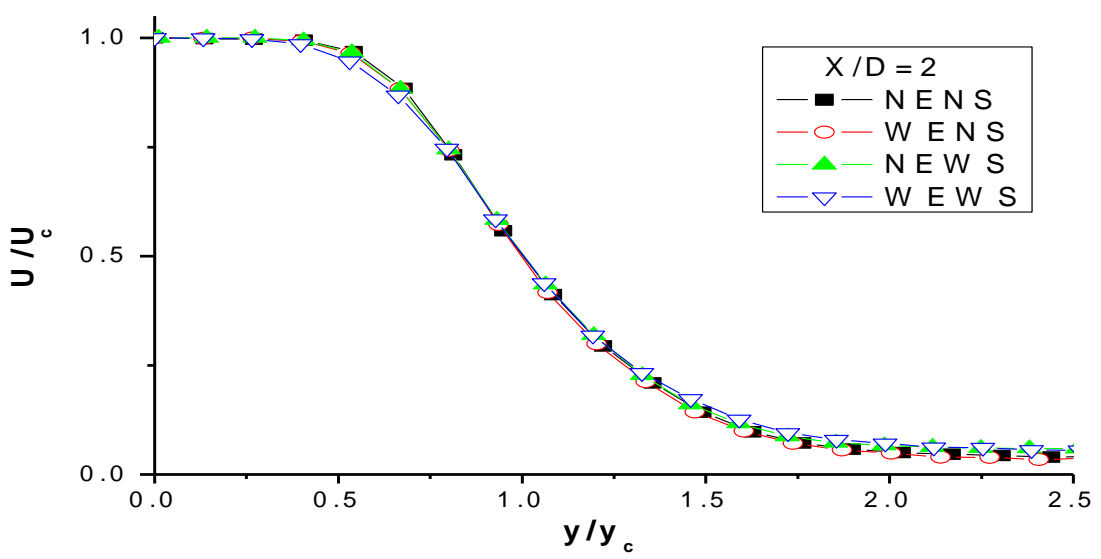

d)

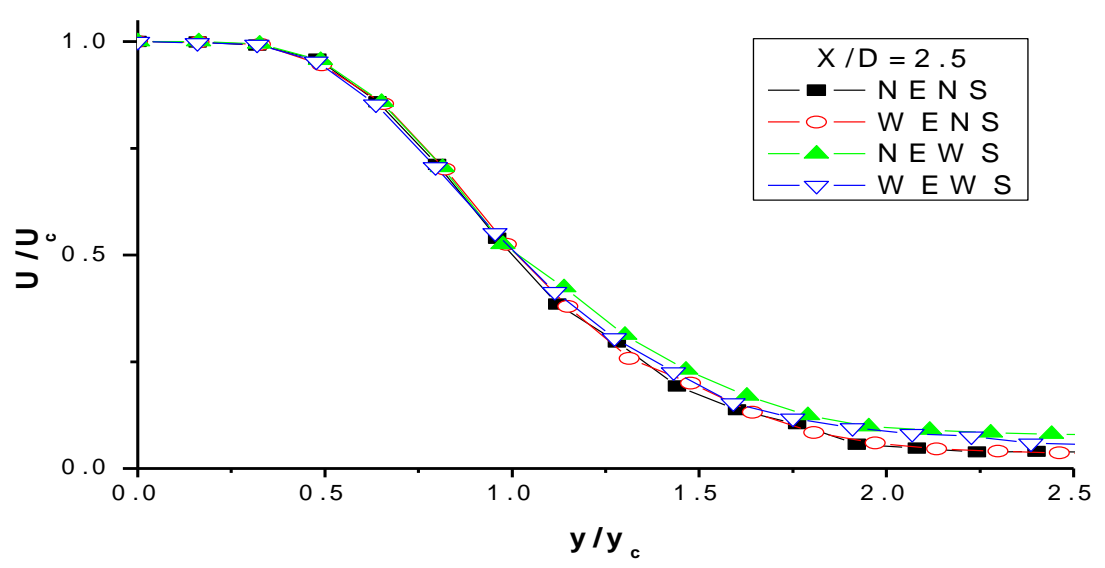

e)

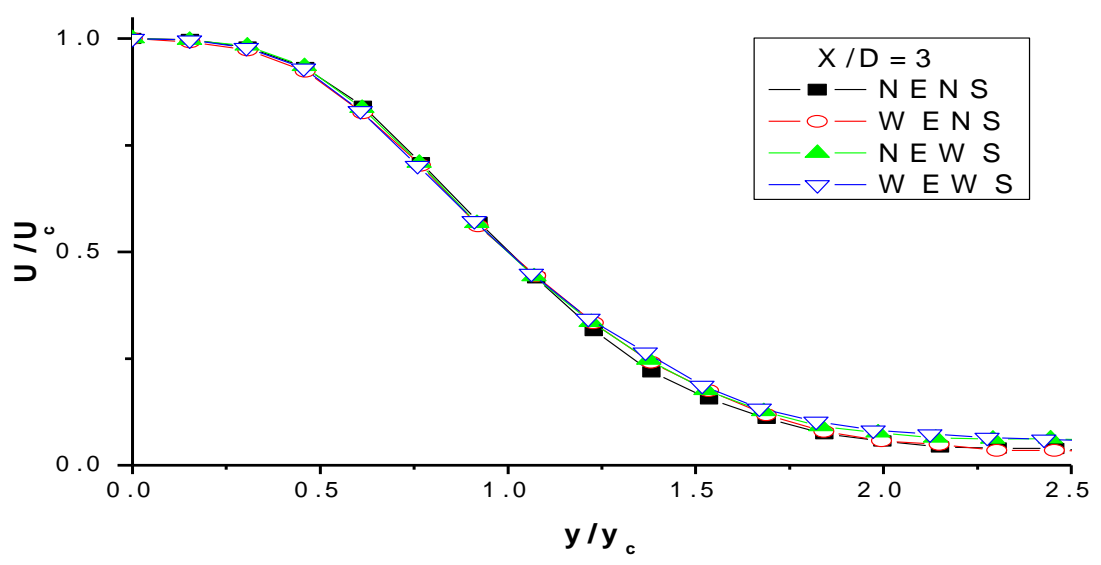

Figure 5.1: Mean streamwise velocity profiles on the central $x y$ plane (cont'd). 
f)

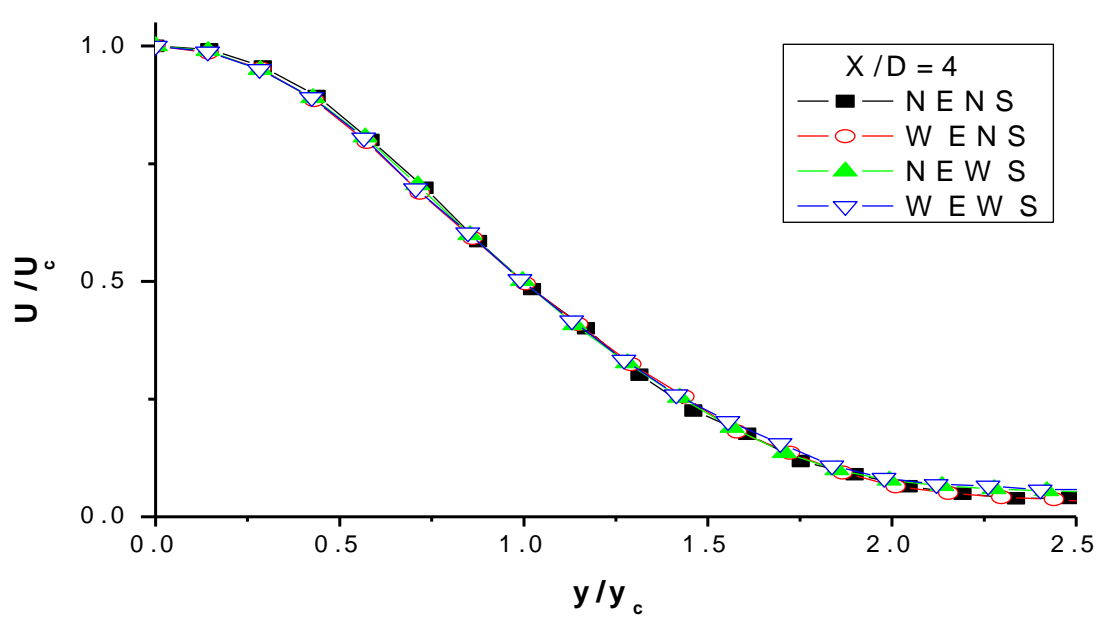

g)

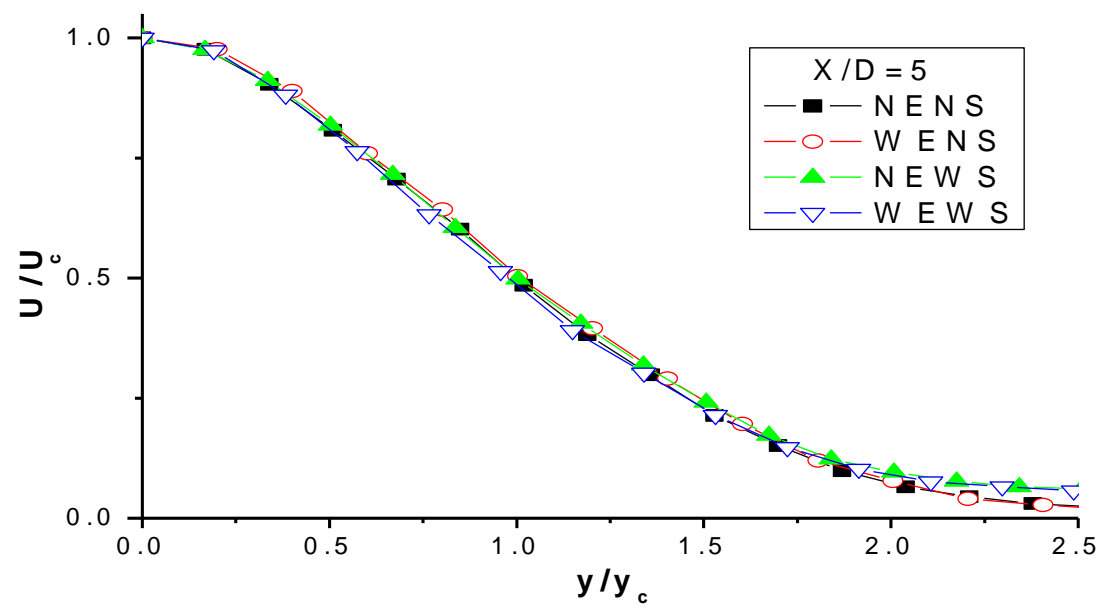

h)

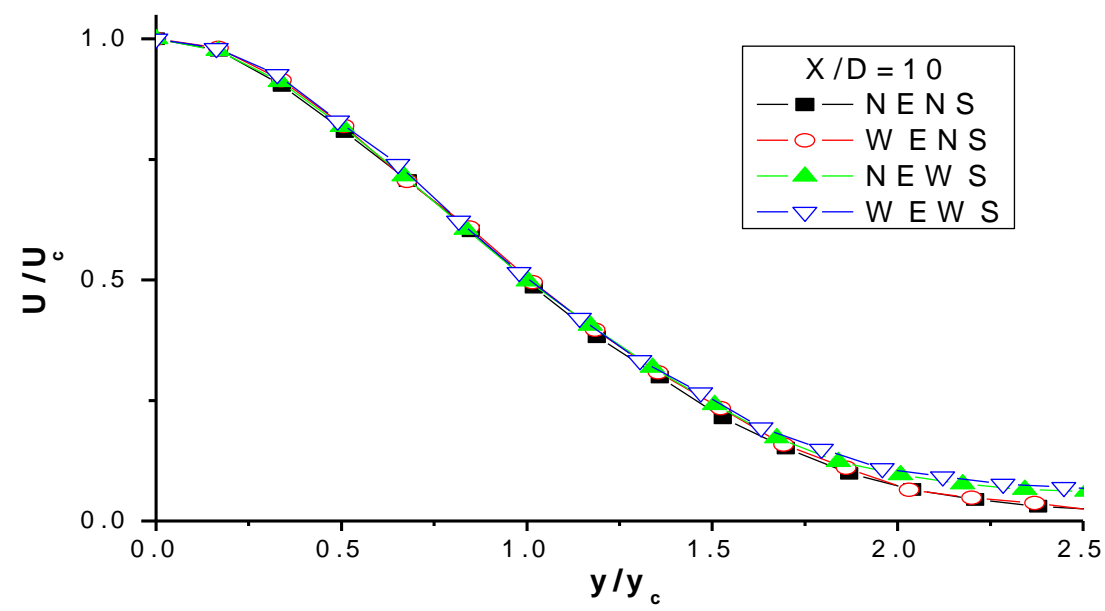

Figure 5.1: Mean streamwise velocity profiles on the central $x y$ plane (cont'd). 
j)

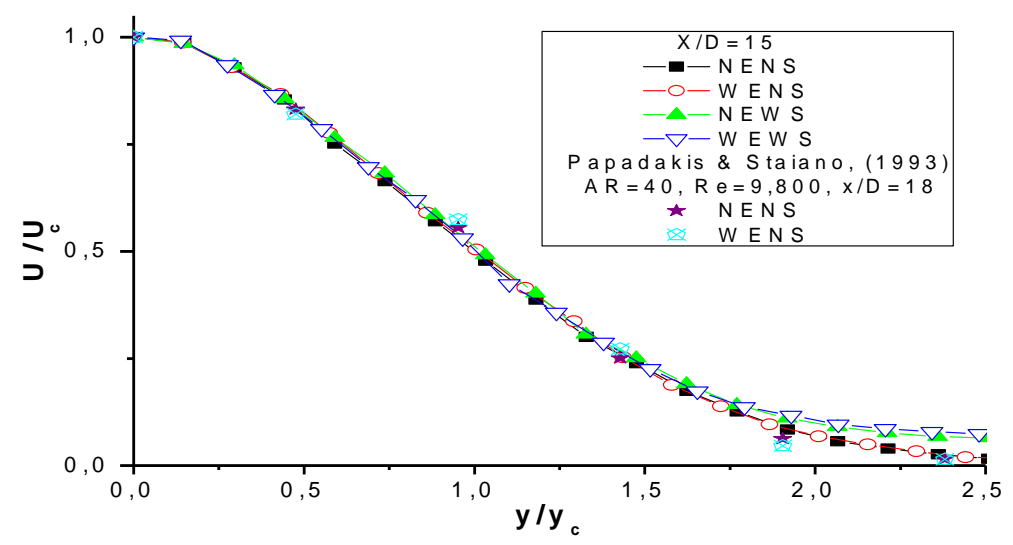

$\mathrm{k})$

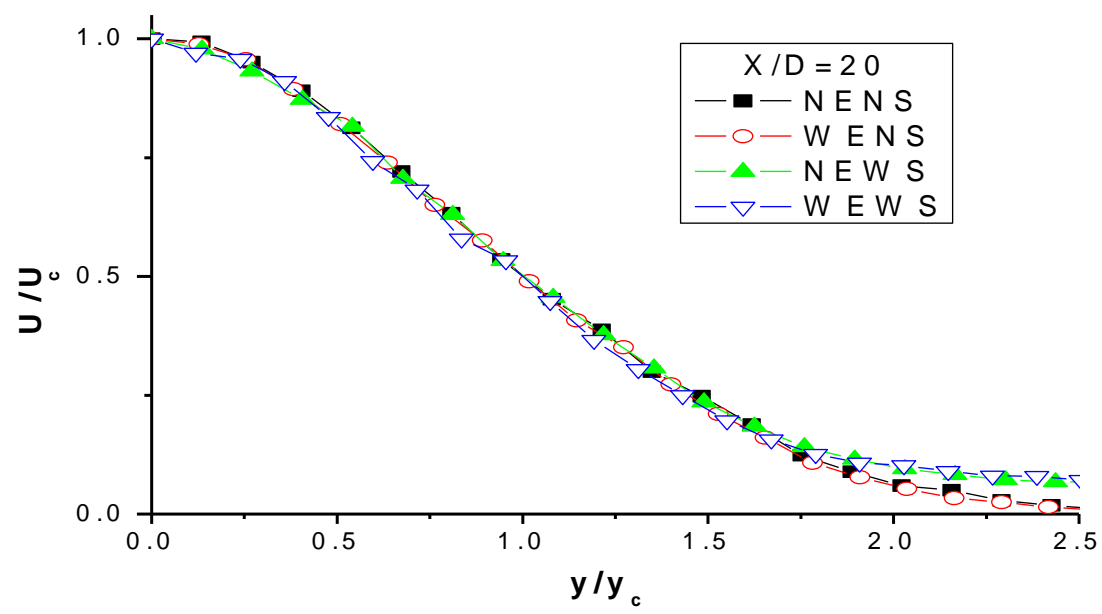

1)

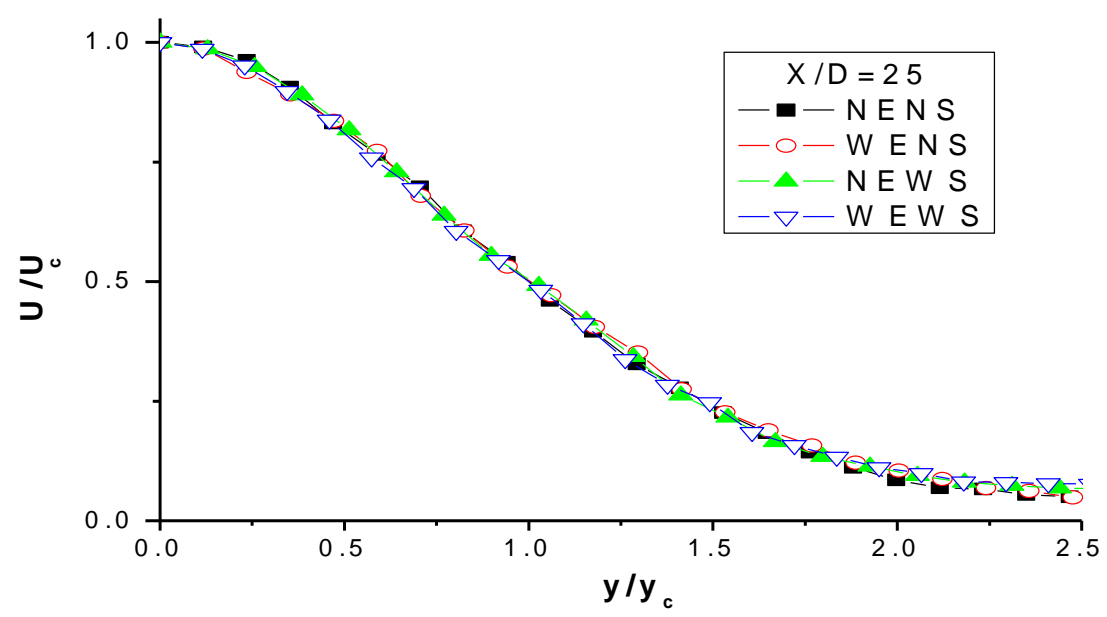

Figure 5.1: Mean streamwise velocity profiles on the central $x y$ plane (cont'd). 
$\mathrm{m})$

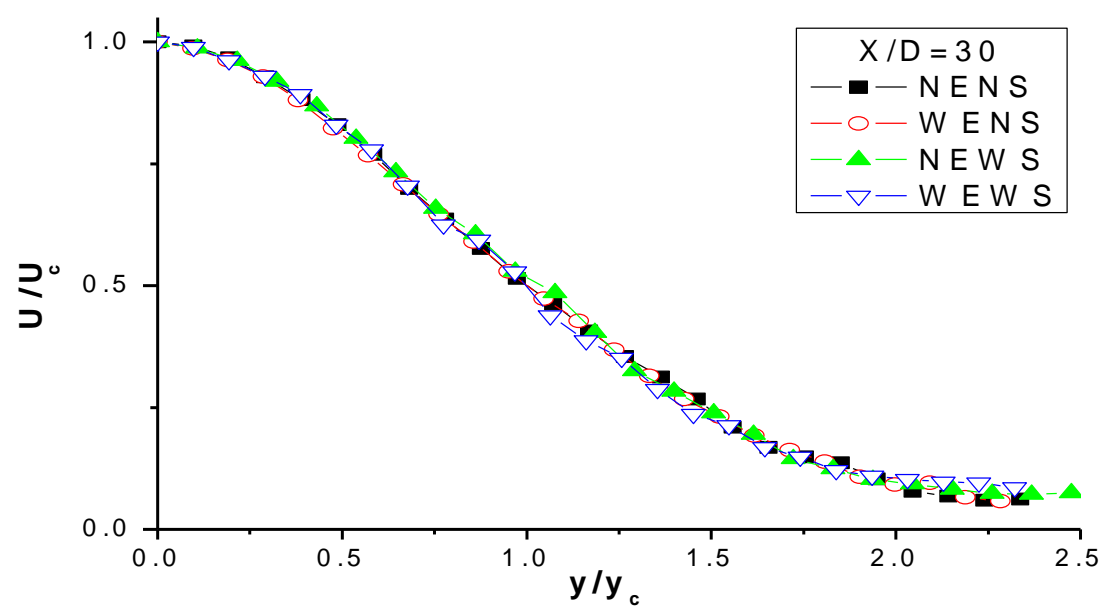

n)

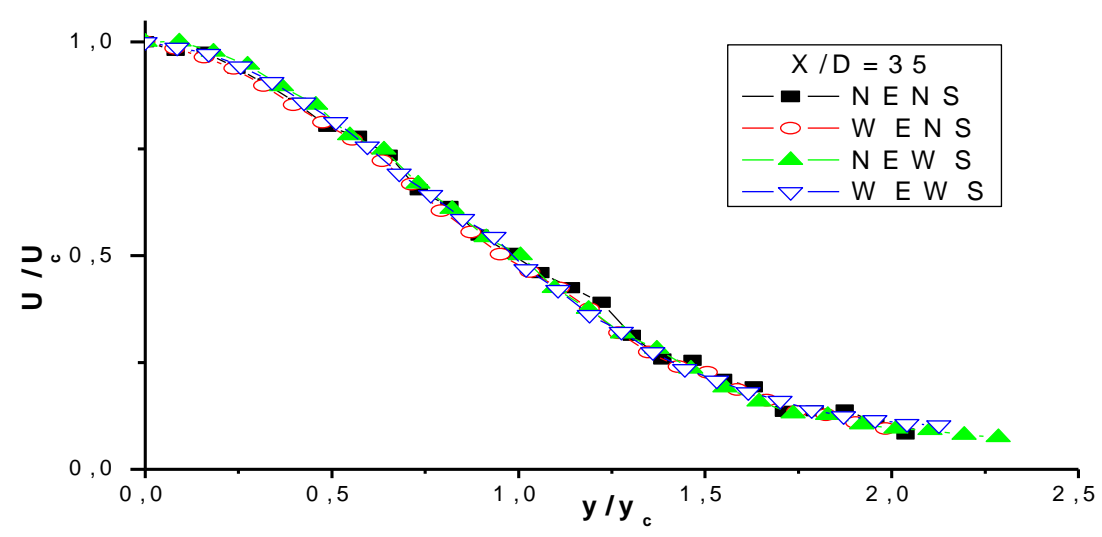

Figure 5.1: Mean streamwise velocity profiles on the central $x y$ plane (cont'd).

\section{b) Similarity of longitudinal mean velocity profiles}

The effect of endplate and sidewalls on the self-similarity of the mean streamwise velocity profiles is investigated. Profiles for NENS, WENS, NEWS and WEWS are shown in Figures 5.2a, b, c and d respectively. As evidenced, in all configurations, the mean streamwise velocity profiles attain self-similarity at approximately similar downstream location $\geq 5 \mathrm{~d}$. These findings are in agreement with previous investigations. Browne et al., (1984) for $A R=19.6$, have found that their mean streamwise velocity profiles attain self-similarity at the same location found in present investigation. Studies on this issue have revealed that the downstream location where the mean streamwise velocity profiles attain self-similarity depends on the initial and boundary conditions. Deo et al., (2008) for $A R=60$, found that 
increasing the Reynolds number from 1,500 to 16,000, the distance where their mean velocity profiles first assumed self-similar was decreased from 20 nozzle width to 5 nozzle width. The nozzle type has been found also to affect the similarity of the streamwise velocity profiles. Deo et al., (2007b) for $A R=72$ and $R e=1.8 \times 10^{4}$ found that when the ratio of exit radii, $r$ and nozzle width, $D$ (i.e. r/D) was increased from zero to 3.6, the location where the mean velocity profiles first assumed self-similarity decreased from 20 nozzle widths to 5 nozzle widths. Besides Reynolds number and nozzle type, aspect ratio has been found to affect the similarity of the streamwise profiles. Deo et al. (2007a) have investigated the effect of aspect ratio for $R e=1.8 \mathrm{x}$ $10^{4}$. They found that with the increase of aspect ratio from 15 to 72 the downstream location where their mean streamwise velocity profiles first assume self-similar was decreased from $x / D=20$ to 5 . The location where their mean streamwise velocity profiles first show self-preservation for $A R=15$ (i.e. $x / D=20$ ) is not in agreement with what we found for the same aspect ratio (i.e. $x / D=5$ ). This difference can be attributed to differences in the experimental conditions between our and their investigations e.g. the nozzle shape i.e. we have used smooth contraction nozzle whereas they have used radially contoured nozzle.

a)

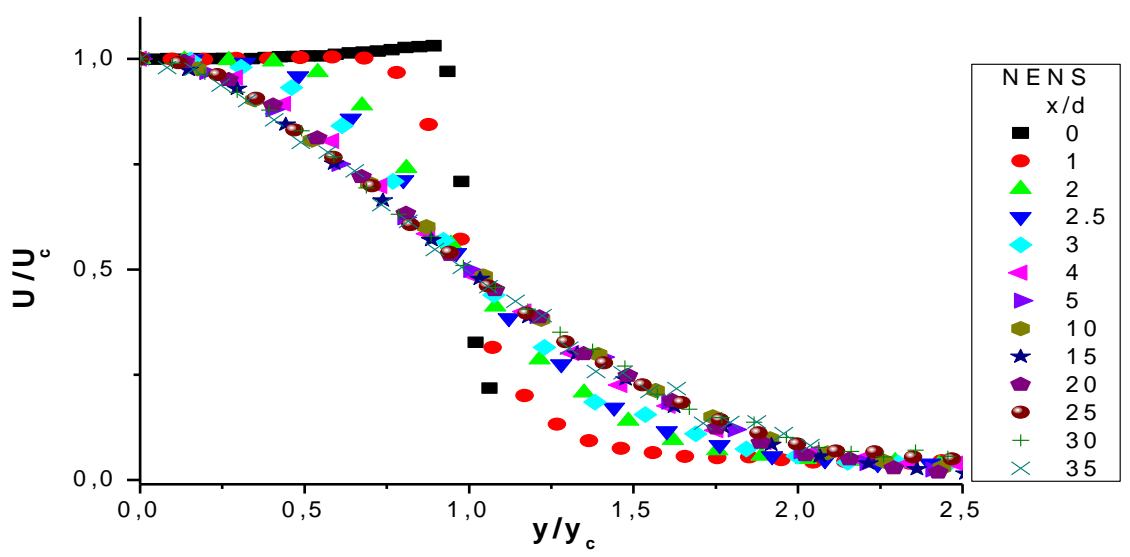

Figure 5.2: Self similarity profiles of mean streamwise velocity on the central $x y$ plane for a) NENS, b) WENS, c) NEWS and d) WEWS. 
b)

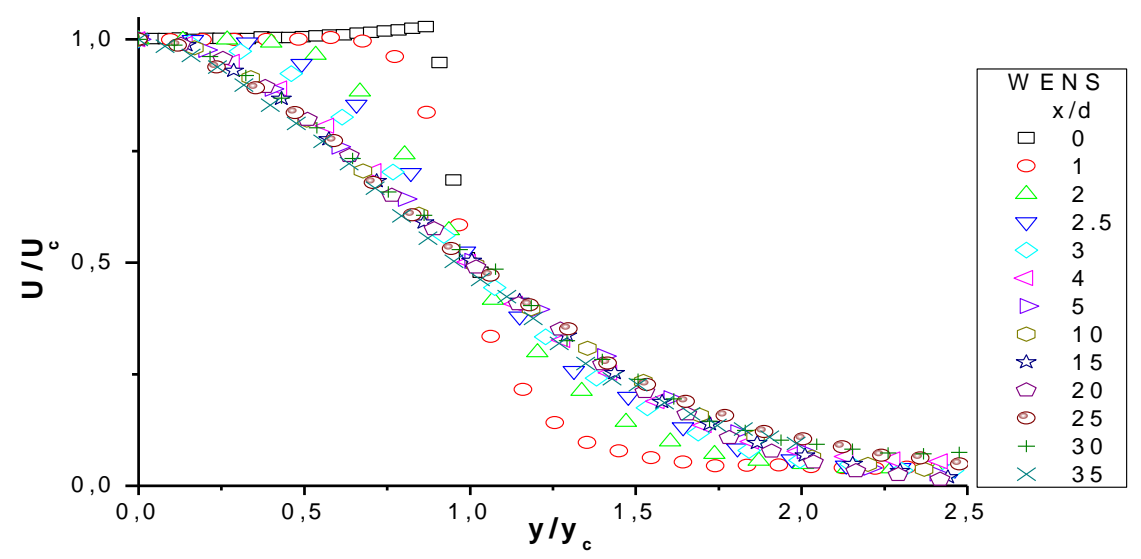

c)

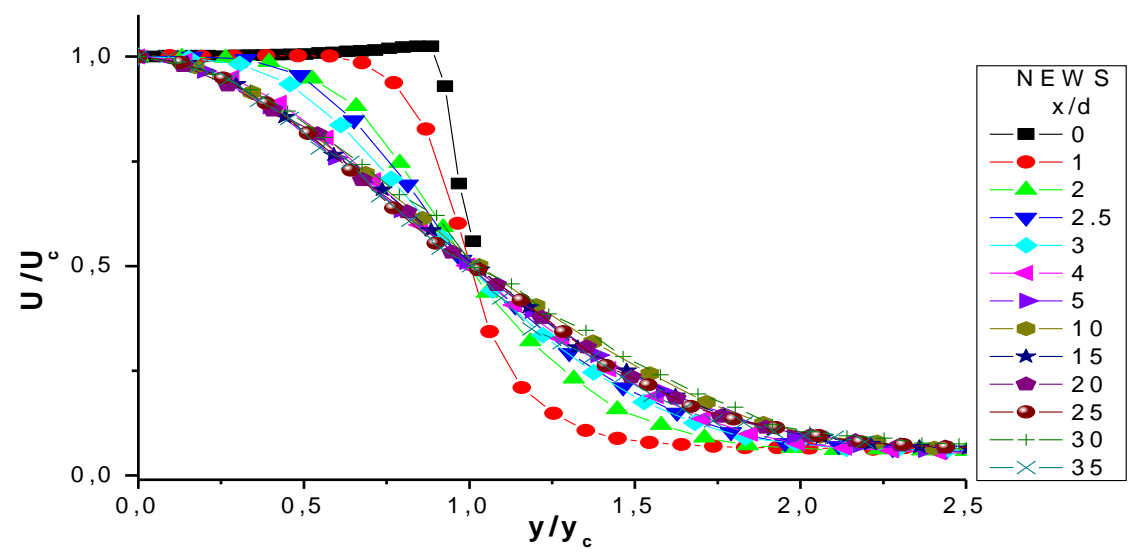

d)

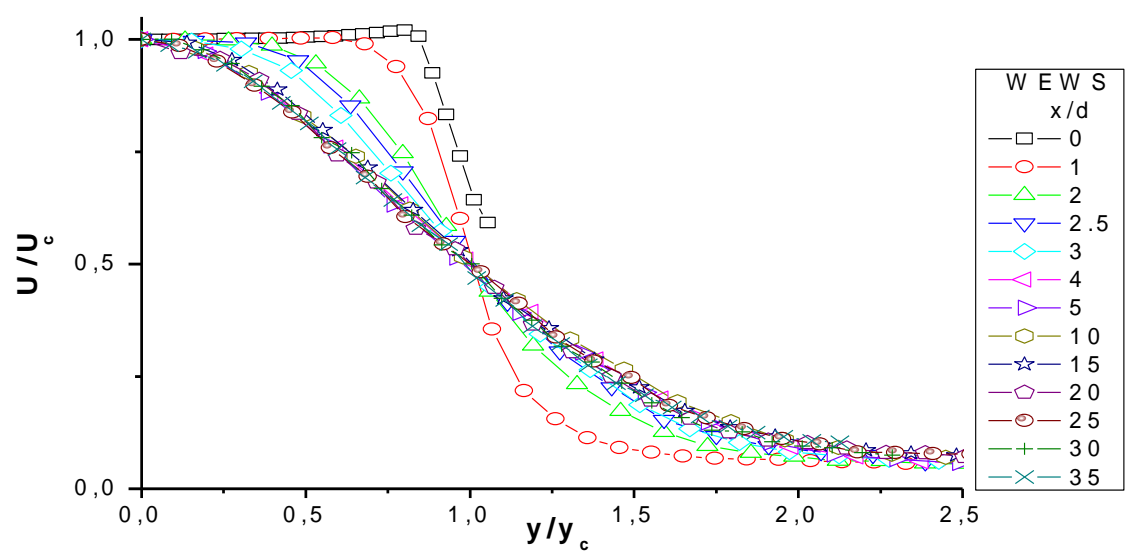

Figure 5.2: Self-similarity profiles of mean streamwise velocity on the central $x y$ plane for a) NENS, b) WENS, c) NEWS and d) WEWS (cont'd). 


\section{c) Spanwise distributions longitudinal mean velocities}

For further exploring the mechanisms produced by the endplate and/or sidewalls, the mean streamwise velocity was measured on the central $x z$ plane at three downstream locations for cases NENS and NEWS at $R e=20,000$. Profiles of $U / U_{c}$ versus $z / D$, where $z$ is the spanwise axis, are shown in Figure 5.3-a, b and c for downstream locations $x / D=2,6$ and 25 respectively. Velocities on the $x z$ plane for the jet configured without sidewalls (NENS and WENS) were measured in the spanwise direction between $-8.5 \leq z / D \leq 8.5$ for $x / D=2$ and 6 and between $-13 \leq z / D \leq 13$ for $x / D=25$. Velocity measurements on the $x z$ plane in the case of the jet NEWS span in the range $-6.75 \leq z / D \leq 6.75$, due to traversing system limitations. Therefore no measurements within the boundary layers developing on the sidewalls are presented.

As shown in Figure 5.5a at $x / D=2$, all jets exhibit similar characteristics having approximately a uniform mean streamwise velocity profile. For the jet without sidewalls a rather thin shear layer is observed. At further downstream locations $(x / D=6$ and 25) differences are observed between the two test cases. At $x / D=6$ the distribution in the cases of the jets with sidewalls presents a local minimum at the central axis and a peak close to the wall. In the cases of the jets without sidewalls a local minimum is observed between the centre and the edge of the jets where a thicker shear layer is developing. At $x / D=25$ both test cases present a local maximum at the centre and a local minimum in between the centre and the edges. For the jet with sidewalls (NEWS) the maximum of the distribution is observed very close to the wall. For the jets without sidewalls the distributions reached close to the shear layer a local maximum of the same order as the values at the centre on the wall. In this case the shear layer thickness has become comparable to the dimensions of the jet in $z$ direction. Undulations of the spanwise profiles of the mean streamwise velocity have been also observed in previous investigations, characterised as saddle back profiles (e.g. Marsters, 1980, Tsuchiya et al., 1986, Quinn, 1992). 
a)

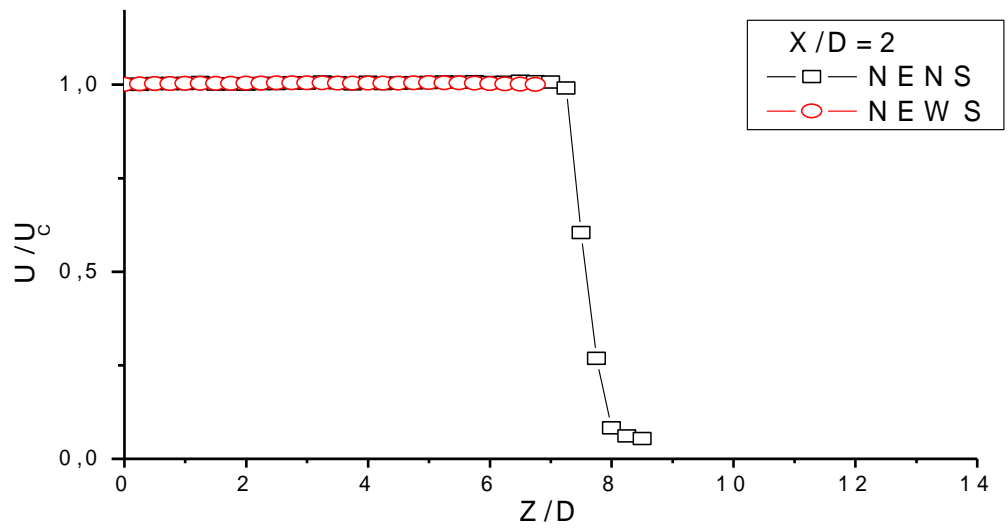

b)

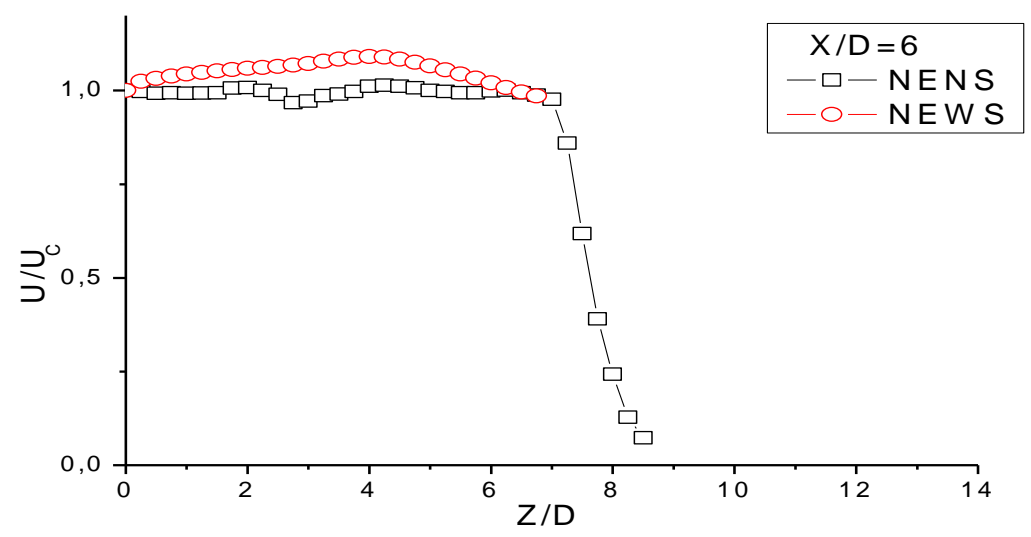

c)

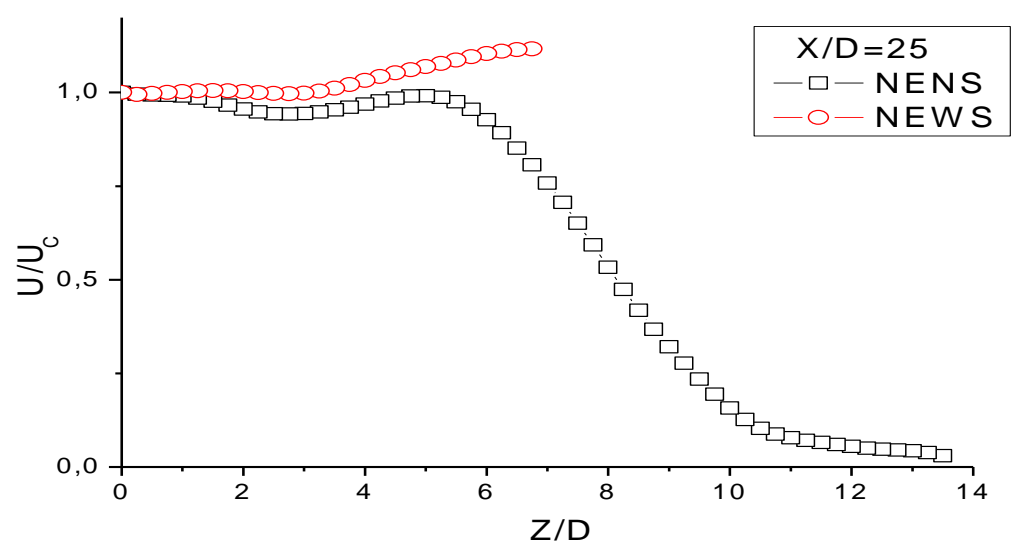

Figure 5.3: Mean streamwise velocity profiles in the central $x z$ plane. 


\subsubsection{Lateral mean velocity}

\section{a) Transverse distributions of lateral mean velocities}

The transverse distributions of the lateral mean velocity in $x y$-plane are presented in Figures 5.4-a, b, c, d, e, f, g, h, j, k, l, m and $\mathrm{n}$ for downstream location $x / D=0,1,2$, $2.5,3,4,5,10,15,20,25,30$ and 35 respectively. They are normalized to the mean streamwise velocity at the center of each location.

In all test cases a distribution of outward positive velocities is observed starting from the centreline (or the edge of the potential core) and reaching beyond the shear layer, its peak moving similarly for all test cases from the shear layer towards the centreline as the distance from the nozzle is increasing. The value of the peak depends on the configuration. The absence of sidewalls is characterized by higher outward velocities and insignificant effect of the presence or absence of the endplate (cases NENS, WENS). When the sidewalls are installed peaks are lower, but the presence of the endplate (WEWS) has an effect leading to noticeably higher values in comparison to the case with no endplate (NEWS). Further outside the shear layer the lateral mean velocity distributions indicate an inward flow, taking negative values. These trends have been evidenced by other investigators (e.g. Gutmark \& Wygnansi, 1976, Krothapalli etal., 1981). We have found that the absence of sidewalls makes the presence of the endplate insignificant and the lateral velocity attains low negative values of almost the same level in both cases (NENS, WENS). The presence of sidewalls is associated with higher negative velocities in this area. Again the presence of the endplate does play a role (WEWS) leading to lower negative velocities in comparison to the case with no endplate (NEWS).

a)

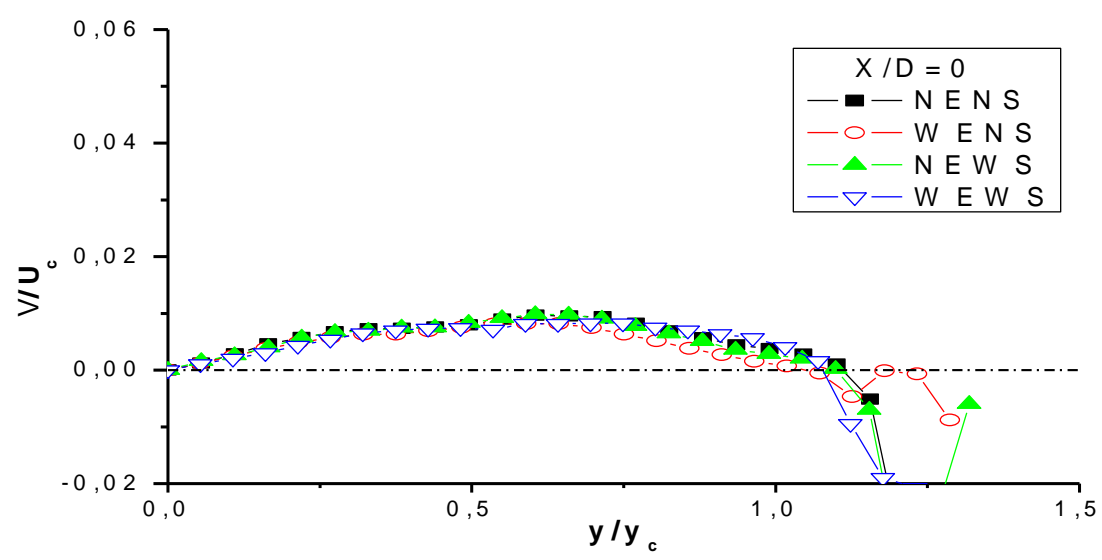

Figure 5.4: Mean lateral velocity profiles on the central $x y$ plane. 
b)

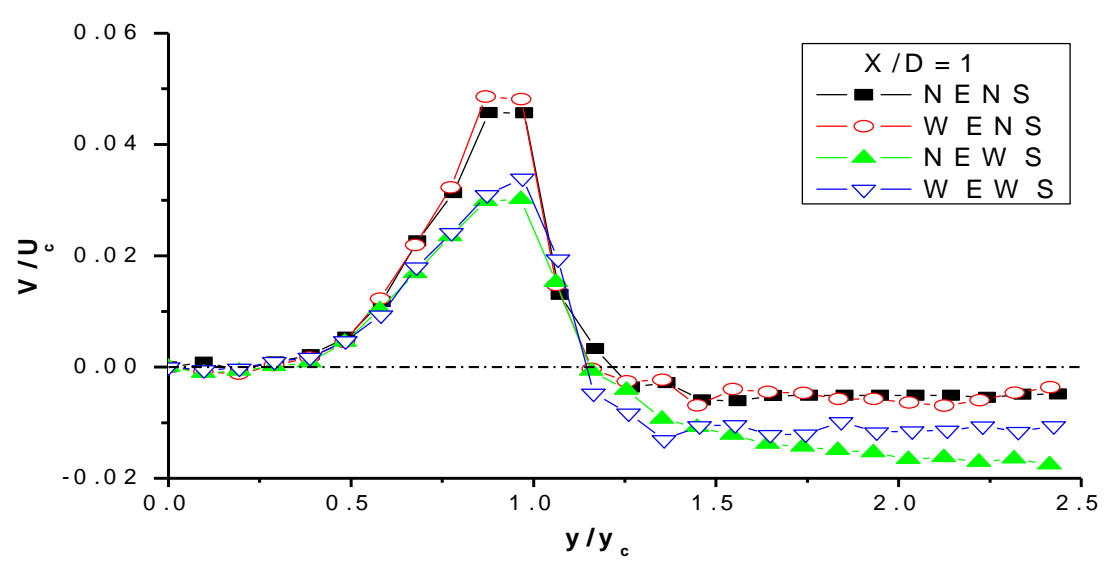

c)

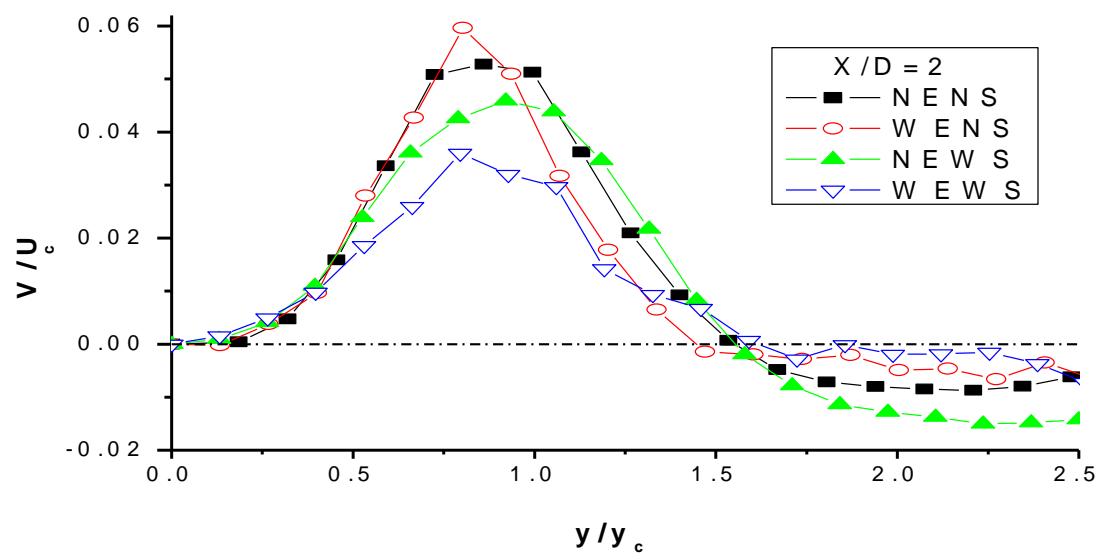

d)

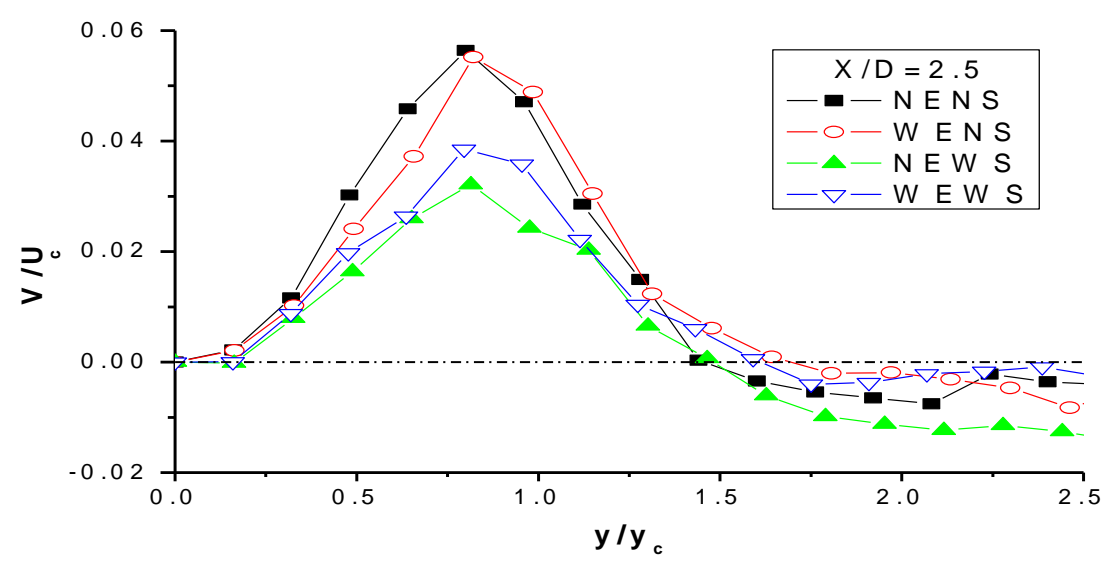

Figure 5.4: Mean lateral velocity profiles on the central $x y$ plane (cont'd). 
e)

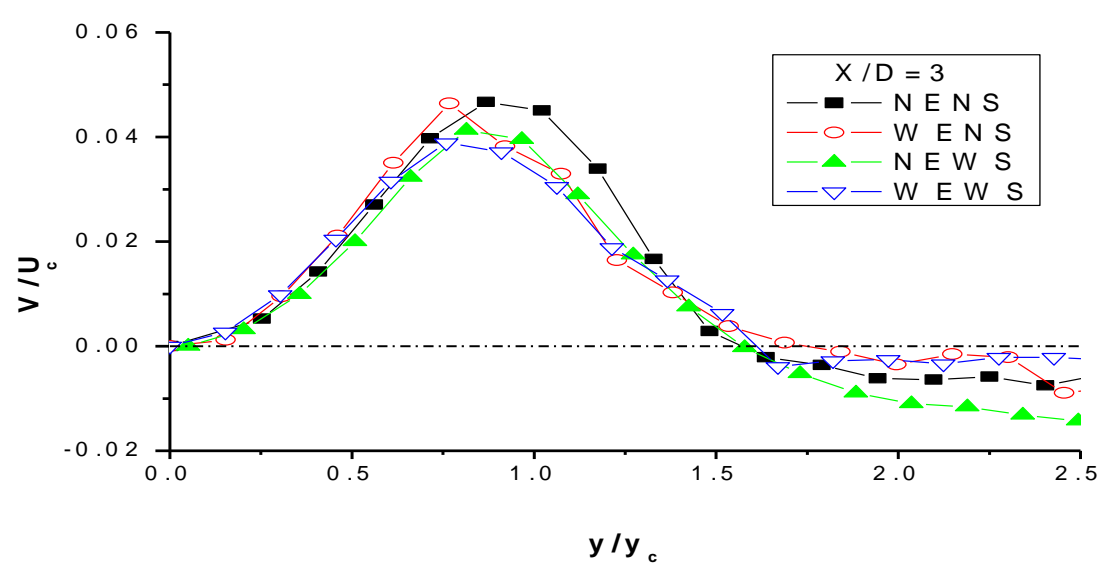

f)

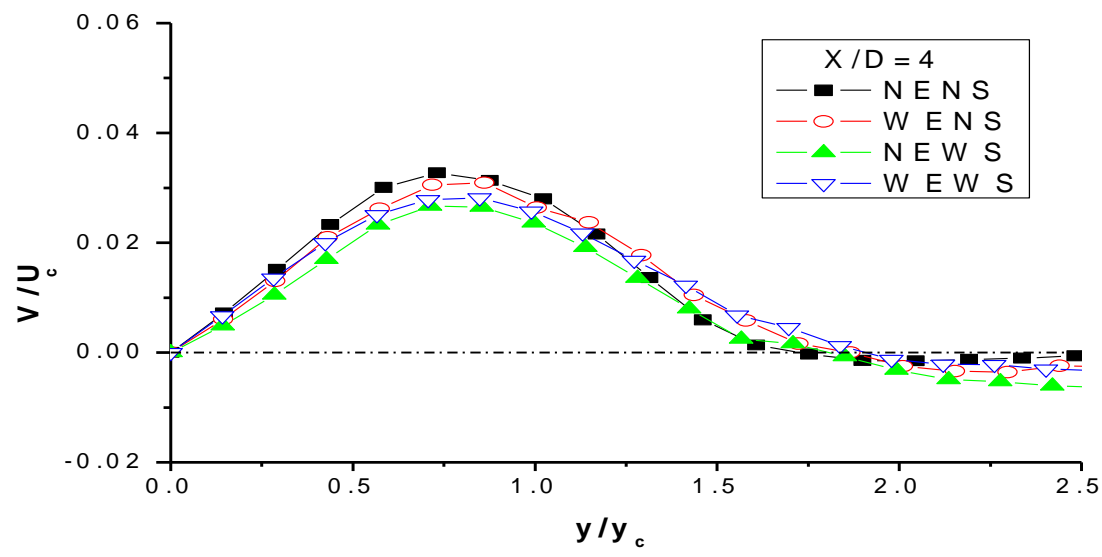

g)

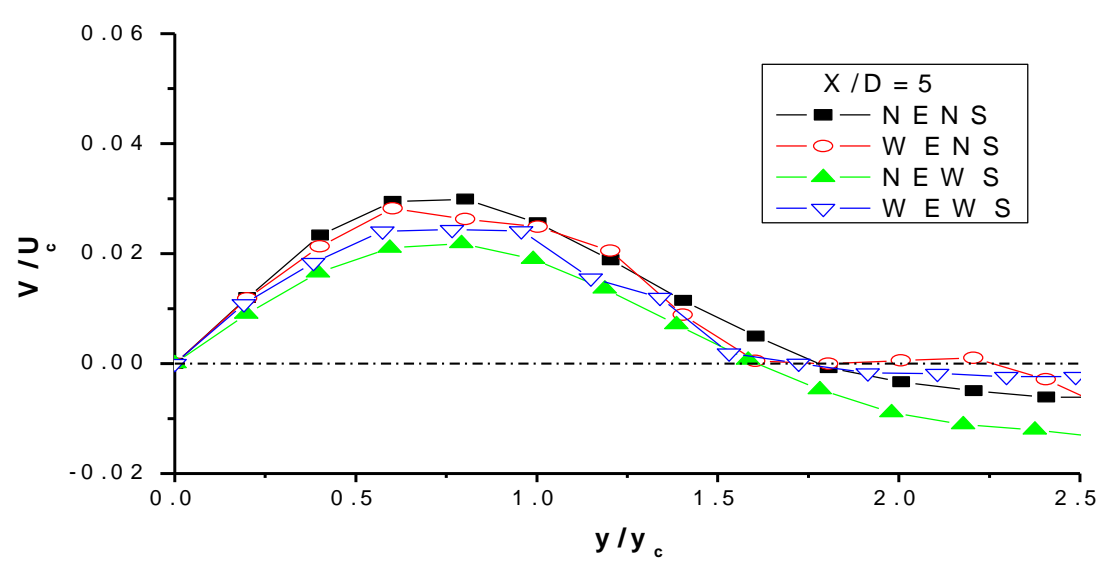

Figure 5.4: Mean lateral velocity profiles on the central $x y$ plane (cont'd). 
h)

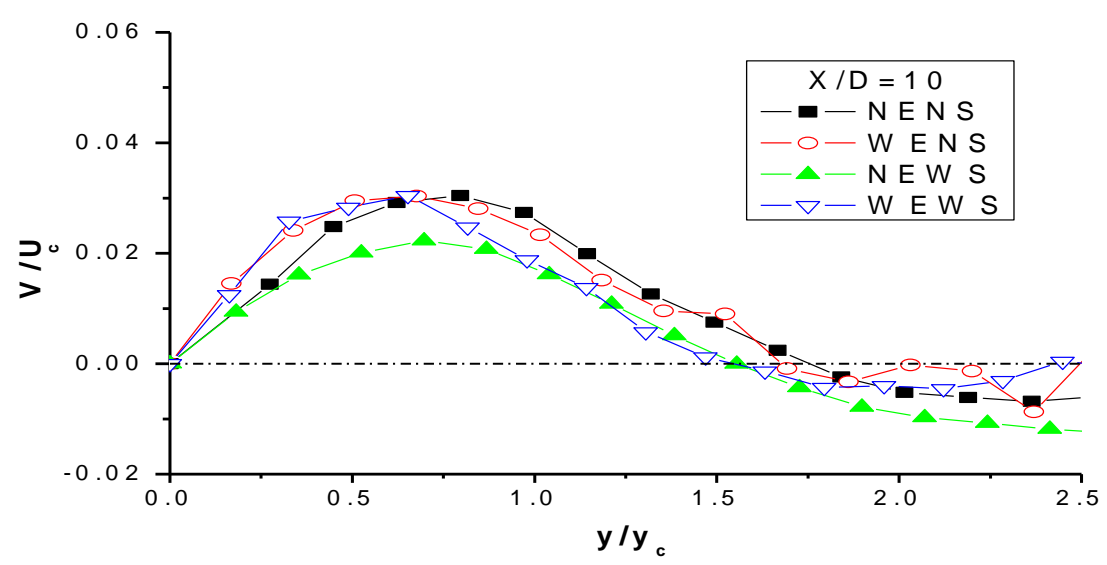

j)

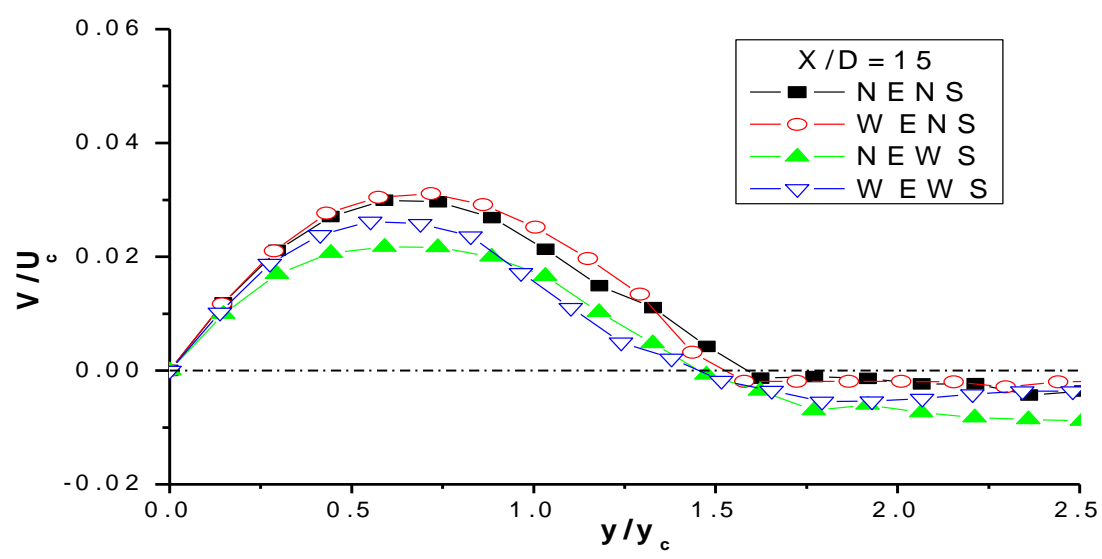

k)

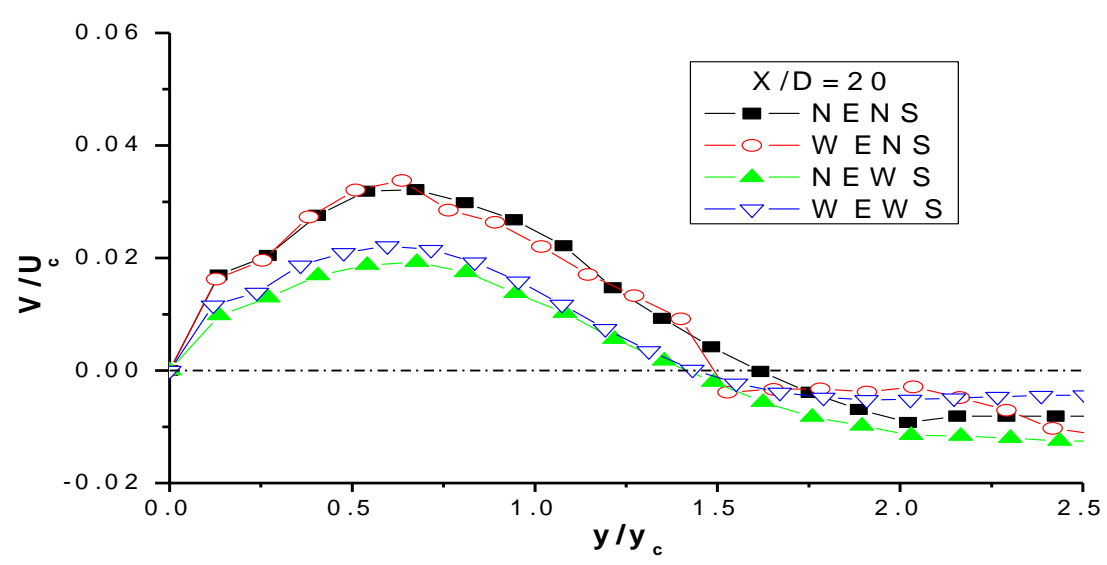

Figure 5.4: Mean lateral velocity profiles on the central $x y$ plane (cont'd). 
1)

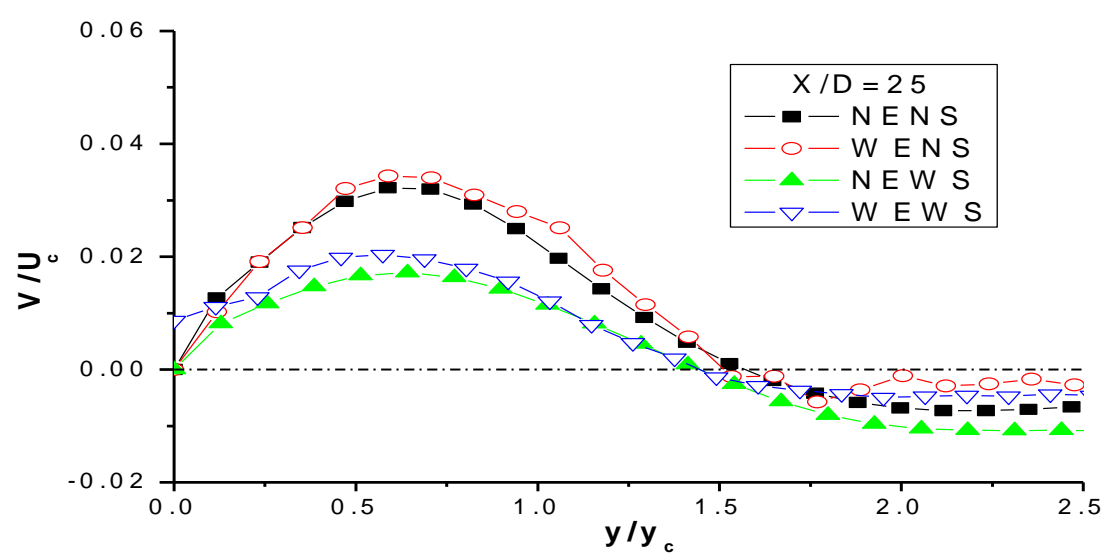

m)

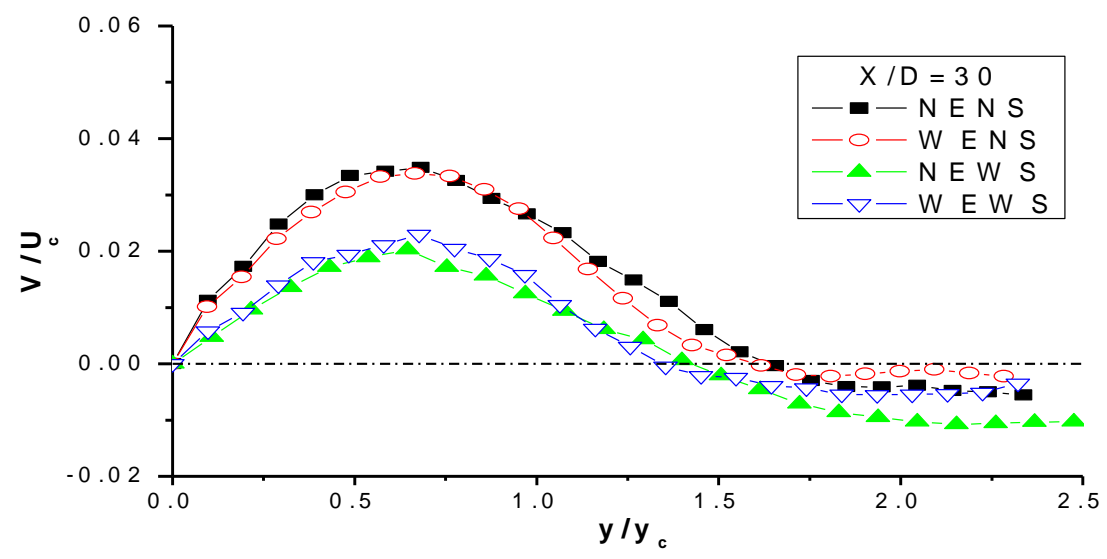

n)

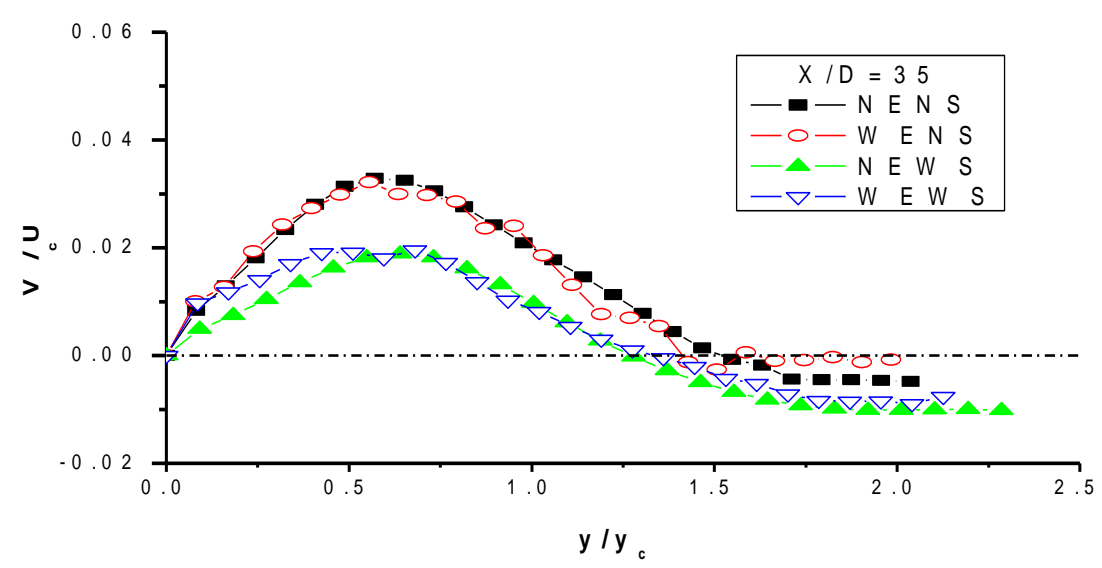

Figure 5.4: Mean lateral velocity profiles on the central $x y$ plane (cont'd) 


\section{b) Similarity of lateral mean velocity profiles}

Figures 5.5-a, b, c and d examine the location where the mean lateral velocity profiles first assume self-similarity for cases NENS, WENS, NEWS and WEWS respectively. As was discussed in the previous section, the presence of the sidewalls (NEWS, WEWS, Figures $5.5 \mathrm{c}$ and d) has led to smaller mean lateral velocity at the shear layer compared to the fee jet cases (NENS, WENS, Figures 5.5a and b). The presence of the endplate in the presence of sidewalls (WEWS, Figure 5.5d) has led to higher mean lateral velocity values at the shear layer whereas smaller negative ones at the edges of the jet. Based on these findings we can say that the presence of the sidewalls or both sidewalls and endplate impose an effect on the mean lateral velocity profiles.

a)

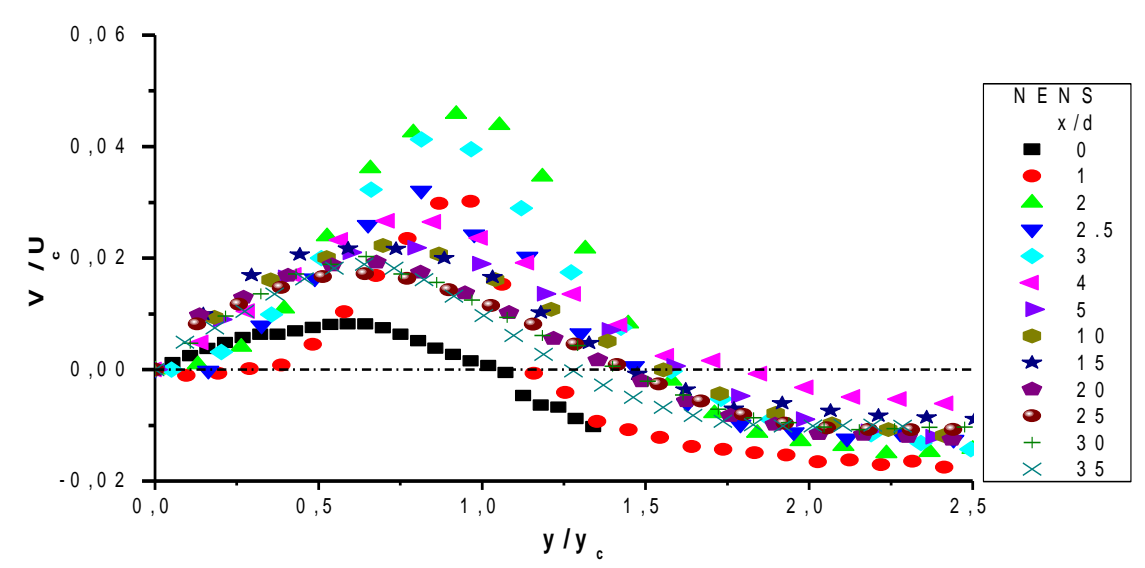

b)

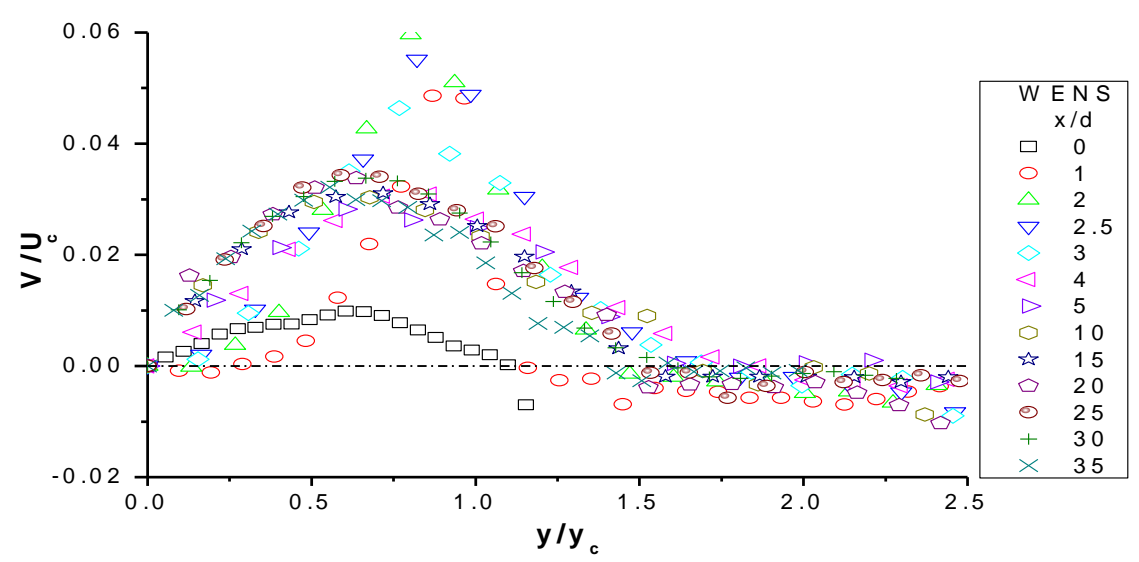

Figure 5.5: Self similarity profiles of mean lateral velocity on the central $x y$ plane a) NENS, b) WENS, c) NEWS and d) WEWS. 
c)

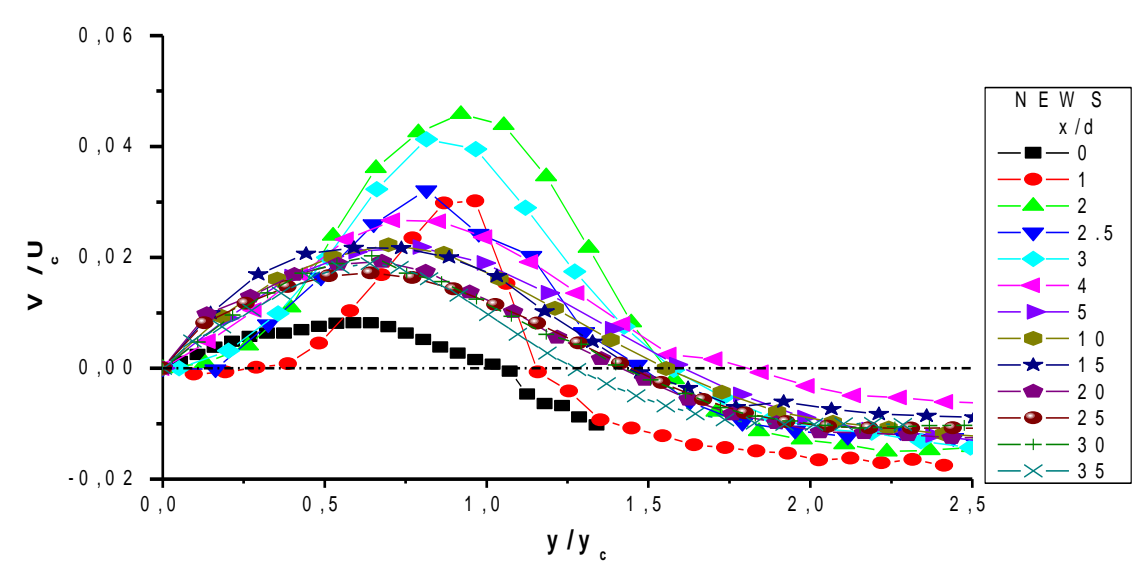

d)

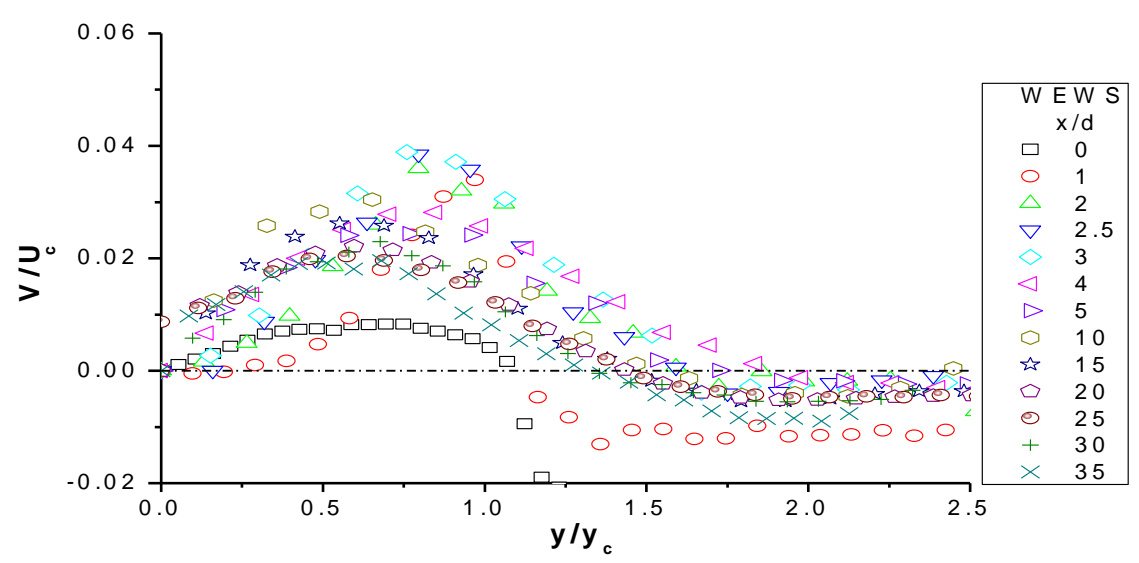

Figure 5.5: Self similarity profiles of mean lateral velocity on the central $x y$ plane for a) NENS, b) WENS, c) NEWS and d) WEWS (cont'd).

\subsubsection{Jet's spread rate}

The spreading of a planar jet is customarily characterised by the downstream growth of the jet half-velocity width, $y_{c}$, defined previously, which is expected to vary linearly with the streamwise coordinate, $x$, beyond a certain distance from the jet exit. This linear evolution is usually described with a relation of the form $y_{d} / D=$ $A_{1}\left[x / D+A_{2}\right]$.

The slope, $A_{l}$, is the spread rate of the jet, and is depending on nozzle geometry (e.g. Deo et al. 2007b) and aspect ratio (e.g. Quinn, 1992, Deo et al., 2007a), Reynolds number (e.g. Namer \& Ötügen, 1988, Suresh et al., 2008, Deo et al., 2008) and sidewalls (e.g. Hitchman et al., 1990, Deo et al., 2007c). $A_{2}$ is the spread virtual origin associated with the location of a corresponding imaginary line source of momentum. 
The evolution of the half velocity width for the four test cases for $R e=20,000$ is presented in Figure 5.6. All measurements almost collapse in the first stages of development, within or just outside the potential core, whereas minor differences may be identified further downstream. The spreading parameters, $A_{1}$ and $A_{2}$ deduced from Figure 5.6 for $x / D \geq 10$ have been included in table 5.1. The virtual origin is in all cases close to the actual jet exit, on the negative side for the cases without sidewalls (NENS, -0.17 D and WENS, $-0.18 D$ ) and on the positive one for the cases with sidewalls (NEWS, $0.14 \mathrm{D}$ and WEWS, $0.15 \mathrm{D}$ ).

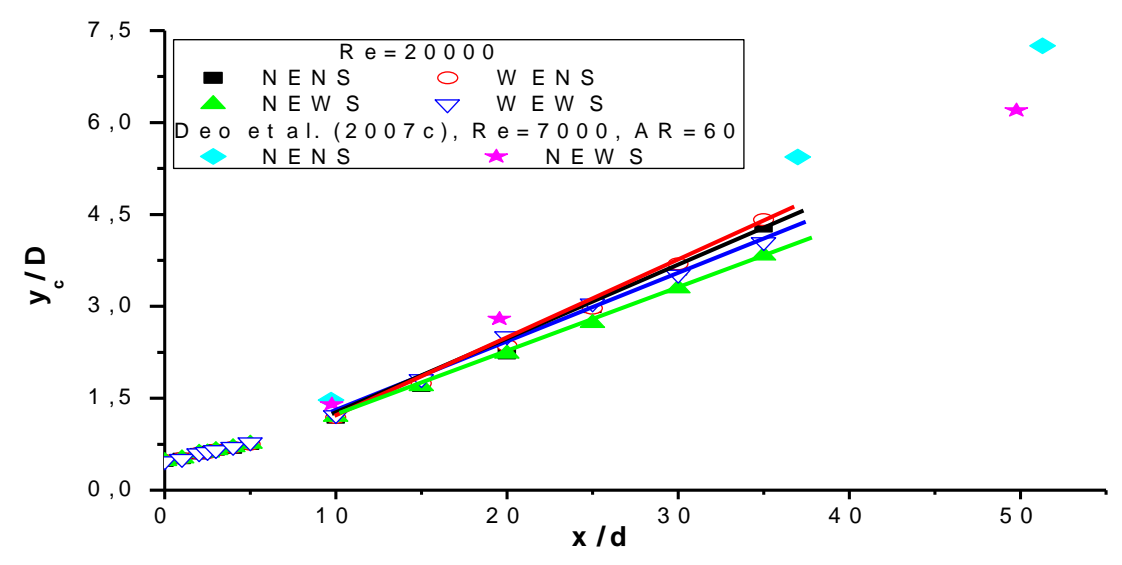

Figure 5.6: Jet half-velocity widths in the stream wise direction

Considering the effect of the sidewalls on the free jet (NENS and NEWS), it is seen that the jet with no endplate and no sidewalls (NENS) spreads faster having a spread rate $A_{1}=0.126$, compared to the jet with no endplate and with sidewalls (NEWS), $A_{l}=0.105$. These findings agree with those of Deo et al., $(2007 \mathrm{c})$, in their investigation on the influence of the sidewalls on a plane jet (without endplate) of $A R=60$ and $R e=7,000$, where similar trends were observed. They found that the jet without sidewalls (NEWS) spreads at a faster rate of 0.14 compared to 0.11 for the jet with sidewalls. Results of Deo et al., (2007c) are included in Figure 5.6 for comparison. These findings are also in good agreement with Hitchman et al., (1990) who obtained a spread rate of 0.106 for the jet without sidewalls and 0.083 for the jet with sidewalls.

Studying the effect of endplate on the free jets (NENS, WENS), we have found that, the two jets spread nearly in the same rate (NENS 0.126, WENS 0.129). This 
indicates that insignificant effect of the endplate on the spread of the studied jet. These findings agree with the findings of Papadakis \& Staiano, (1993). Their LDA measurements have also, shown insignificant effect of endplates on the spreading rate of the jet $(A R=40, R e=9800)$, whereas Kotsovinos, (1978) studying a plane jet out of a wall associated jets with endplates with slower spreading rates in line with a reduction in momentum. Abdel-Rahman et al., (1998) studying the effect of the endplate on an axisymmetric jet, using $L D A$ concluded that a reduction in momentum, slower spread rate but similar decay are associated to the jet with endplate.

Based on these findings, it is may be concluded that, the spread rate, $A_{l}$, seems to depend more on the presence or absence of the sidewalls and less to the presence or absence of the endplate, which seems to have an effect only when the sidewalls are installed.

\subsubsection{Entrainment and mass flow}

The entrainment of the jet can be inferred from the total mass (volume) flow rate, $m$, i.e. the velocity integral across the jet multiplied by the constant density. The growth of the mass flow rate relatively to the exit value of the reference NENS configuration, $m_{0}$, for all four configurations is presented in Figure 5.7.

The initial mass flow rate of all configurations is almost identical. In the first stages corresponding to the potential region the values collapse and thereafter the trends distinguish the jets incorporating sidewalls (NEWS, WEWS) from those without sidewalls (NENS, WENS). In the range $x / D=5-25$ the jets incorporating sidewalls entrain ambient fluid more effectively than the jets with no sidewalls whereas this trend is reversed at successive stations and the mass flow rate increase declines for the jets with sidewalls (NEWS, WEWS). The differences between the configurations incorporating sidewalls and those which do not may be attributed to the two dimensional characteristics imposed by the sidewalls. In the early stages the mass flow at the central $x y$ plane is increasing slower in the absence of sidewalls due to the spreading in the spanwise direction. When the $3 \mathrm{D}$ jets with no sidewalls are beginning to attain an axisymmetric structure, the flow rate due to the rearrangement 
of the flow is increasing faster than for the two dimensional jets with sidewalls, which gradually lose their potential to entrain ambient fluid.

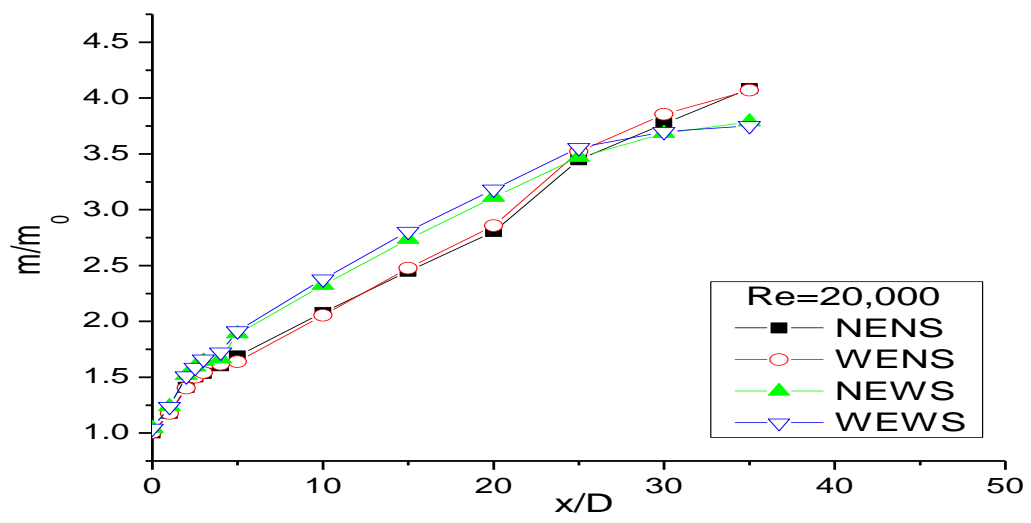

Figure 5.7: Mass flow rate ratio along the jets

\subsubsection{Momentum growth in the axial direction}

The corresponding curves for the momentum flow, $M$, i.e. the momentum integral across the jet, normalised by the exit value of the reference NENS configuration, $M_{0}$, are depicted in Figure5.8 The initial momentum of the configurations making use of sidewalls is higher probably due to the confinement of the jet preventing the expansion of the jet and the diffusion of momentum in the spanwise direction. Again the values corresponding to both jets without sidewalls (NENS, WENS) are almost the same along the jet. They remain close to the initial momentum flow until $x / D=20$, rising thereafter gradually to higher values indicating a positive increase of the momentum flow, which may be attributed in accordance with the increase of the mass flow rate to the rearrangement of the $3 \mathrm{D}$ flow field towards an axisymmetric structure. In the presence of sidewalls momentum flow values are again close to the initial ones until $x / D=20$ but thereafter those of the jet without the endplate (NEWS) increase marginally over one and attain a declining shape reaching one at the furthest location measured, whereas the values corresponding to the jet with the endplate (WEWS) remain always lower than the initial ones and attain a declining shape at the furthest locations, indicating a reduction of the momentum in the far field in accordance with the investigations mentioned previously. 


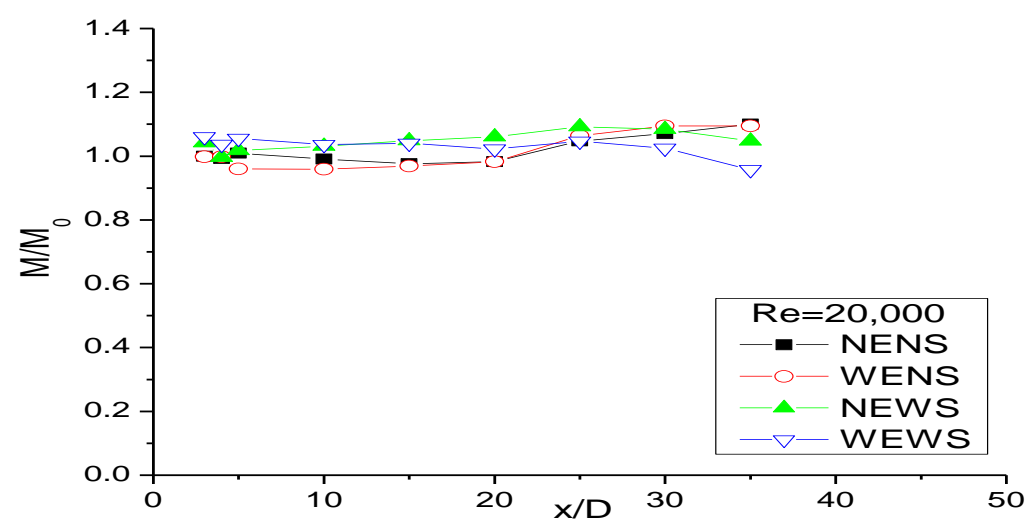

Figure 5.8: Momentum flow ratio along the jets

\subsubsection{Axial mean velocity decay at various Reynolds numbers}

The decay behaviour of the centreline mean velocity presented by $\left(U_{0} / U_{c}\right)^{2}$ versus $x / D$ (where $U_{0}$ is the mean streamwise velocity at the exit plane), for three Reynolds numbers, $R e=10,000,20,000$ and 30,000, for all four test configurations is shown in Figures 5.9-a, b and c respectively.

Assessing the effects of the endplate on jet without sidewalls (NENS and WENS), when the sidewalls are not present, the endplate seems to have an insignificant impact on the decay characteristics for all Reynolds numbers tested (configurations NENS and WENS). The decay rate increases from $R e=10,000$ to $R e=20,000$ and then shows a small reduction from $R e=20,000$ to $R e=30,000$ for both configurations, as if it had reached saturation. The decay curves in Figures 5.9-a, b and c are hardly distinguishable and the corresponding values of $B_{1}$ and $B_{2}$ remain very close for both configurations (see also table 5.1 and Figure 5.10). Insignificant influence of the endplate on the decay characteristics has been also found for jets of $A R=40$, without sidewalls, for $R e=9800$ by Papadakis \& Staiano, (1993). The results of Papadakis \& Staiano, (1993) are included in Figure 5.9a. 
a)

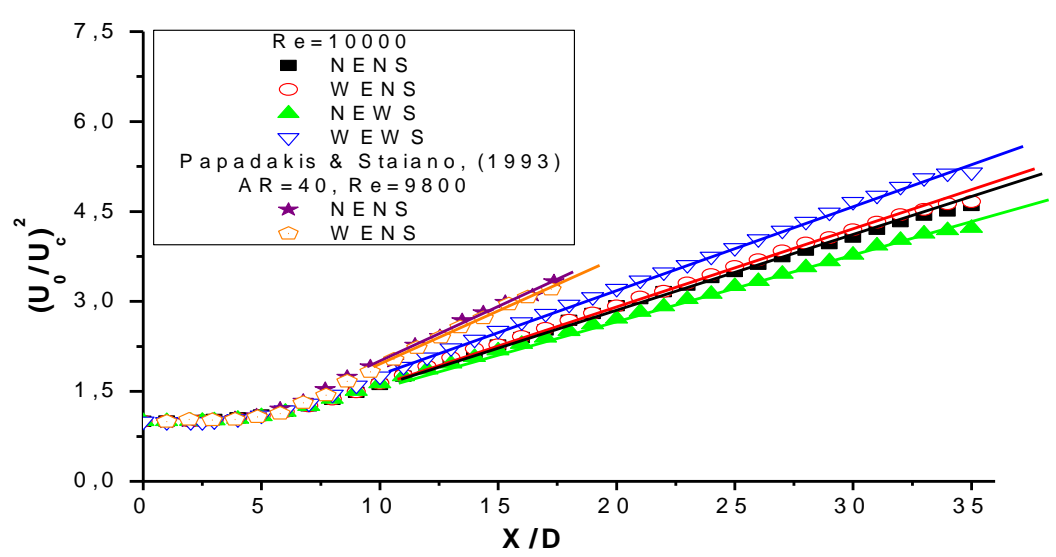

b)

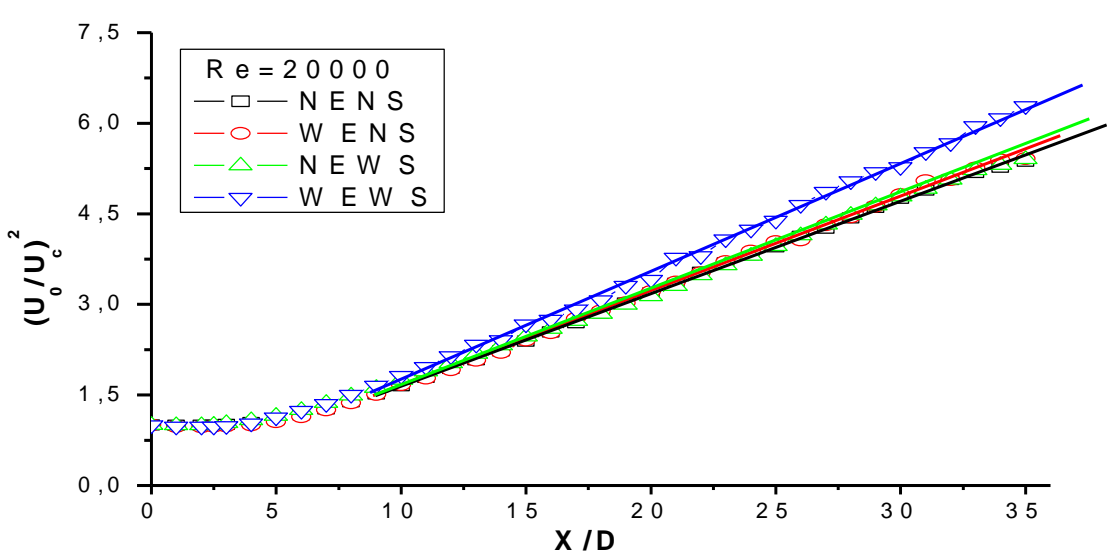

c)

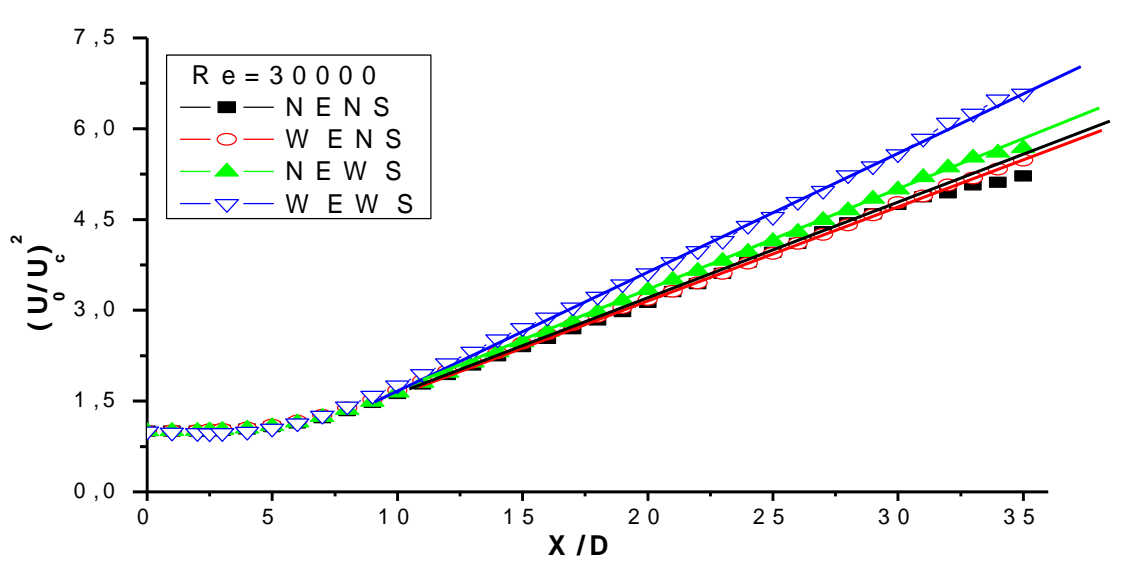

Figure 5.9: Axial mean velocity decay at different Reynolds numbers, a) $R e=10,000$, b) $R e=20,000$ and c) $R e=30,000$. 
Focusing on the effects of the sidewalls on jet without endplate (NENS and NEWS), we will find that at $R e=10,000$, the centreline velocity in the case of NENS decays faster than NEWS after the potential region, in accordance with Deo et al., (2007c) findings. A switch is observed at $R e=20,000$ regarding the trends of the two test cases. The curves in Figure 5.9-b almost collapse, and a scarcely discernible difference is observed in a small area after the potential core where the centreline velocity of the jet with no sidewalls takes higher values. At $R e=30,000$ the relation observed at $R e=10,000$ has been completely reversed, and the centreline mean velocity of the jet with no sidewalls decays at a slower rate than in the presence of the sidewalls, in agreement with the work of Hitchman et al., (1990), in which slower decay rate was associated with the jet without sidewalls.

The Reynolds dependence and the effect of the sidewalls in this series of experiments is notably different to that found by Namer \& Ötügen, (1988) for moderate Reynolds number jets $(R e=1,000-7,000)$ of $A R=56$ without sidewalls and Deo et al., (2008) in a large range of Reynolds numbers $(R e=1,500-57,500)$ for jets of aspect ratios $A R=36$ or 60 with sidewalls. Both these investigations indicate a decrease in $B_{1}$ as well as in $A_{l}$ with increasing Reynolds number. Taking into account the findings of Deo et al., (2007a) the different dependence of $B_{1}$ on Reynolds number should be mainly attributed to the low aspect ratio of the currently presented jets and the resulting interplay between the effects of $A R, R e$ and that of sidewalls. In the presence of sidewalls the endplate has a significant effect on the Reynolds number dependence of $B_{1}$ and $B_{2}$. As indicated by the values in table 5.1 and the graphs in Figure 5.10a, $B_{1}$ is increasing with the Reynolds number in a similar manner to that of the NEWS jet but the corresponding curve has been shifted to higher values. It is interesting to note that corresponding although opposite, trends are observed in Figure 5.10-b for the dependence of $B_{2}$ on configuration and Reynolds number. 
a)

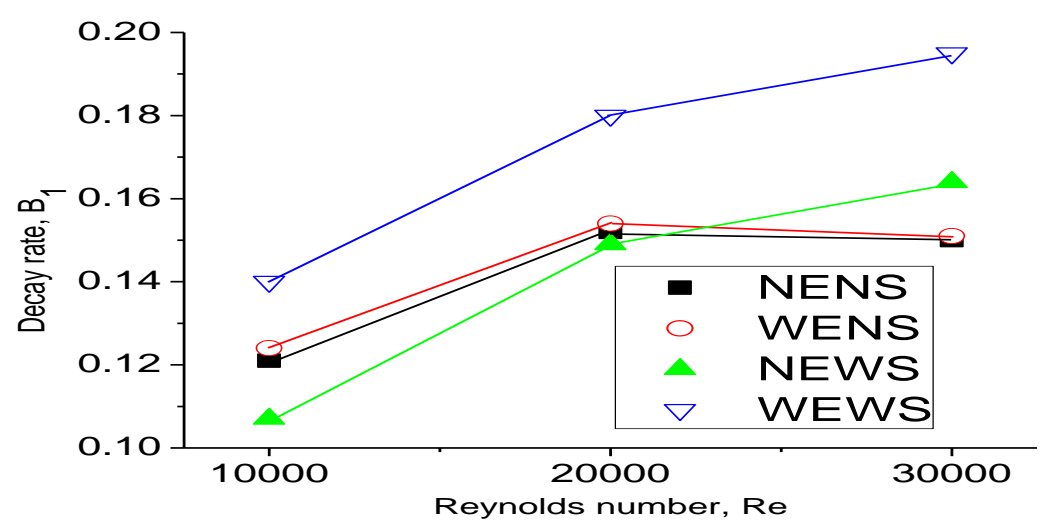

b)

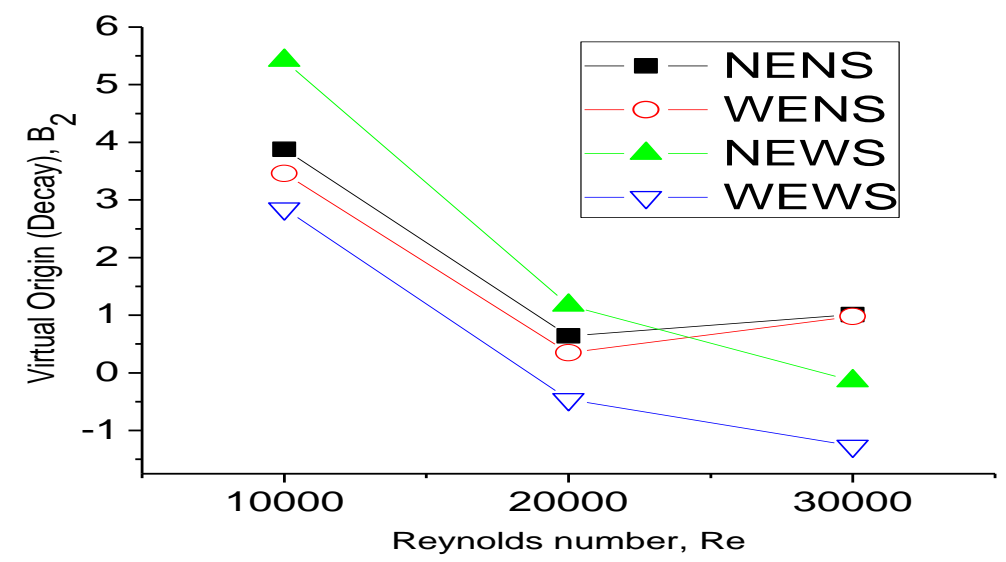

Figure 5.10: Axial mean velocity decay characteristics at different Reynolds numbers, a) decay rate, $B_{1}$, b) virtual origin, $B_{2}$. 
Table 5.1 Jet characteristics including far field mean stream wise velocity decay, $B_{1}$, virtual origin, $B_{2}$, spread rate, $A_{1}$, spread virtual origin, $A_{2}$ and nozzle type of the present investigation compared to other published data

\begin{tabular}{|c|c|c|c|c|c|c|c|c|}
\hline Source & $\begin{array}{c}\text { Aspect } \\
\text { ratio, } \\
\text { AR }\end{array}$ & $\begin{array}{c}\text { Reynolds } \\
\text { number, } \\
\text { Re }\end{array}$ & $\begin{array}{c}\text { Jet } \\
\text { conditio } \\
\text { n }\end{array}$ & $\begin{array}{c}\text { Decay } \\
\text { rate, } \\
\mathbf{B}_{1}\end{array}$ & $\begin{array}{c}\text { Virtual } \\
\text { Origin, } \\
\mathbf{B}_{2}\end{array}$ & $\begin{array}{c}\text { Spread } \\
\text { rate, } \\
\mathbf{A}_{1}\end{array}$ & $\begin{array}{c}\text { Spread } \\
\text { Virtual } \\
\text { origin, } \\
\mathbf{A}_{2} \\
\end{array}$ & $\begin{array}{c}\text { Nozzle } \\
\text { type }\end{array}$ \\
\hline $\begin{array}{l}\text { Present } \\
\text { investigation }\end{array}$ & 15 & $\begin{array}{l}10,000 \\
20,000\end{array}$ & $\begin{array}{l}\text { NENS } \\
\text { WENS } \\
\text { NEWS } \\
\text { WEWS } \\
\text { NENS } \\
\text { WENS } \\
\text { NEWS } \\
\text { WEWS } \\
\text { NENS } \\
\text { WENS } \\
\text { NEWS } \\
\text { WEWS }\end{array}$ & $\begin{array}{c}0.121 \\
0.124 \\
0.107 \\
0.14 \\
0.152 \\
0.154 \\
0.149 \\
0.180 \\
0.150 \\
0.151 \\
0.164 \\
0.195\end{array}$ & $\begin{array}{c}3.88 \\
3.46 \\
5.42 \\
2.85 \\
0.64 \\
0.35 \\
1.17 \\
-0.46 \\
1.01 \\
0.98 \\
-0.14 \\
-1.27\end{array}$ & $\begin{array}{l}0.126 \\
0.129 \\
0.105 \\
0.112\end{array}$ & $\begin{array}{r}-1.39 \\
-1.41 \\
1.34 \\
1.36\end{array}$ & $\begin{array}{c}\text { Smooth } \\
\text { contracti } \\
\text { on }\end{array}$ \\
\hline $\begin{array}{c}\text { Gutmark \& } \\
\text { Wygnanski, } \\
(1976)\end{array}$ & 38 & 30,000 & WEWS & 0.17 & & 0.10 & & $\begin{array}{c}\text { Smooth } \\
\text { contracti } \\
\text { on }\end{array}$ \\
\hline $\begin{array}{l}\text { Krothapalli et } \\
\text { al., } \\
(1981)\end{array}$ & 16.7 & 12,000 & NENS & & & 0.109 & & $\begin{array}{c}\text { Rect. } \\
\text { channel }\end{array}$ \\
\hline $\begin{array}{c}\text { Namar \& } \\
\text { Ötügen, } \\
\text { (1988) }\end{array}$ & 56 & $\begin{array}{l}1,000 \\
6,000 \\
7,000\end{array}$ & NENS & $\begin{array}{l}0.239 \\
0.175\end{array}$ & & $\begin{array}{l}0.179 \\
0.098\end{array}$ & & $\begin{array}{c}\text { Smooth } \\
\text { contracti } \\
\text { on }\end{array}$ \\
\hline $\begin{array}{c}\text { Deo et al., } \\
\text { (2008) }\end{array}$ & 60 & $\begin{array}{c}1,500 \\
\\
3,000 \\
4,300 \\
7,000 \\
10,000 \\
16,500\end{array}$ & NEWS & $\begin{array}{l}0.219 \\
0.187 \\
0.183 \\
0.178 \\
0.172 \\
0.159\end{array}$ & & $\begin{array}{l}0.137 \\
0.125 \\
0.105 \\
0.098 \\
0.088\end{array}$ & & $\begin{array}{l}\text { Radially } \\
\text { contoure } \\
\text { d nozzle }\end{array}$ \\
\hline $\begin{array}{l}\text { Hitchman et al., } \\
\text { (1990) }\end{array}$ & 60 & 7,230 & $\begin{array}{l}\text { WENS } \\
\text { WEWS }\end{array}$ & $\begin{array}{l}0.182 \\
0.222\end{array}$ & & $\begin{array}{l}0.106 \\
0.083\end{array}$ & & $\begin{array}{c}\text { Smooth } \\
\text { contracti } \\
\text { on. }\end{array}$ \\
\hline $\begin{array}{l}\text { Deo et al., } \\
(2007 \mathrm{c})\end{array}$ & 60 & 7,000 & $\begin{array}{l}\text { NENS } \\
\text { NEWS }\end{array}$ & $\begin{array}{l}0.20 \\
0.17\end{array}$ & & $\begin{array}{l}0.14 \\
0.11\end{array}$ & & $\begin{array}{l}\text { Radially } \\
\text { contoure } \\
\text { d nozzle }\end{array}$ \\
\hline $\begin{array}{c}\text { Papadakis \& } \\
\text { Staiano, (1993) }\end{array}$ & 40 & 9800 & $\begin{array}{l}\text { NENS } \\
\text { WENS }\end{array}$ & $\begin{array}{l}0.185 \\
0.182\end{array}$ & $\begin{array}{l}0.632 \\
0.610\end{array}$ & & & $\begin{array}{c}\text { Smooth } \\
\text { contracti } \\
\text { on }\end{array}$ \\
\hline $\begin{array}{l}\text { Abdel-Rahman } \\
\text { et al., (1997) }\end{array}$ & $\begin{array}{c}\text { Axisym } \\
\text { metric }\end{array}$ & $1.32 \times 10^{4^{*}}$ & $\begin{array}{l}\mathrm{NE}^{* *} \\
\mathrm{WE}^{* *}\end{array}$ & $\begin{array}{l}5.95 \\
5.90\end{array}$ & & $\begin{array}{r}0.097 \\
0.08\end{array}$ & & $\begin{array}{l}\text { Round } \\
\text { nozzle }\end{array}$ \\
\hline
\end{tabular}

\footnotetext{
*Reynolds number based on the diameter of nozzle, $D_{r}$, **NE: No Endplate, WE: with Endplate
} 


\subsubsection{Turbulent Velocity Field}

\subsubsection{Longitudinal turbulent velocity}

\section{a) Transverse distributions of longitudinal turbulent velocities normalized to the centreline velocity}

At the exit plane the root-mean square (rms) of the longitudinal velocity fluctuations, $u^{\prime}$, in the shear layer was found to be $3.8 \%$ in the absence of the sidewalls (NENS and WENS), higher than the corresponding value of $3.1 \%$ found in the presence of the sidewalls (NEWS and WEWS) (see Figure 5.11-a). These findings agree with Deo et al. (2007c). They have also found that at the exit nozzle $u^{\prime}$ is $3.6 \%$ and $2.8 \%$ in the absence and presence of the sidewalls respectively. This difference is confirming the influence of the sidewalls even on the very near flow field. Current results indicate that the presence of an endplate has an insignificant effect and the estimates for the root mean square of the streamwise velocity fluctuation $u^{\prime}$, at the exit shear layer can be grouped according to the absence or presence of sidewalls (3.8\% of the mean centreline velocity for NENS and WENS, and 3.1\% for NEWS and WEWS).

The lateral distributions of the streamwise turbulent velocity, $u^{\prime}$, on the central $x y$ plane, for all four configurations at $R e=20,000$, are shown in Figures 5.11-a, b, c, d, e, f, g, h, j, k, 1, m and n 6.8-a, b, c and d at $x / D=0,1,2,2.5,3,4,5,10,15,20,25,30$ and 35 respectively. Peak values are within the range 16-24\% of $U_{c}$, increasing slightly with the distance from the nozzle. In the early stages of development peaks are close to $y / y_{c}=1$ moving towards the centre of the jet at subsequent locations. Values at the centre are quite low in the potential zone increasing gradually downstream and reaching values close to peak values after the merging of the two shear layers.

The presence of the endplate has an insignificant effect when the sidewalls have not been implemented and the measurements for NENS and WENS jets almost collapse at all stations. In the results of the configurations comprising sidewalls (NEWS, WEWS), in the near field, both jets exhibit smaller values at the shear layers and at edges of the jets in comparison to the free jets (NENS, WENS). In the far field, the 
values of longitudinal turbulent velocities of NEWS and WEWS were found to systematically increase in the streamwise direction. This increase is noticeably higher in the presence of the endplate (WEWS). The presence of the endplate in the round jet of Abdel-Rahman et al., (1997) led also to higher values of rms velocity component in the streamwise direction (Figure 7 in their paper).

a)

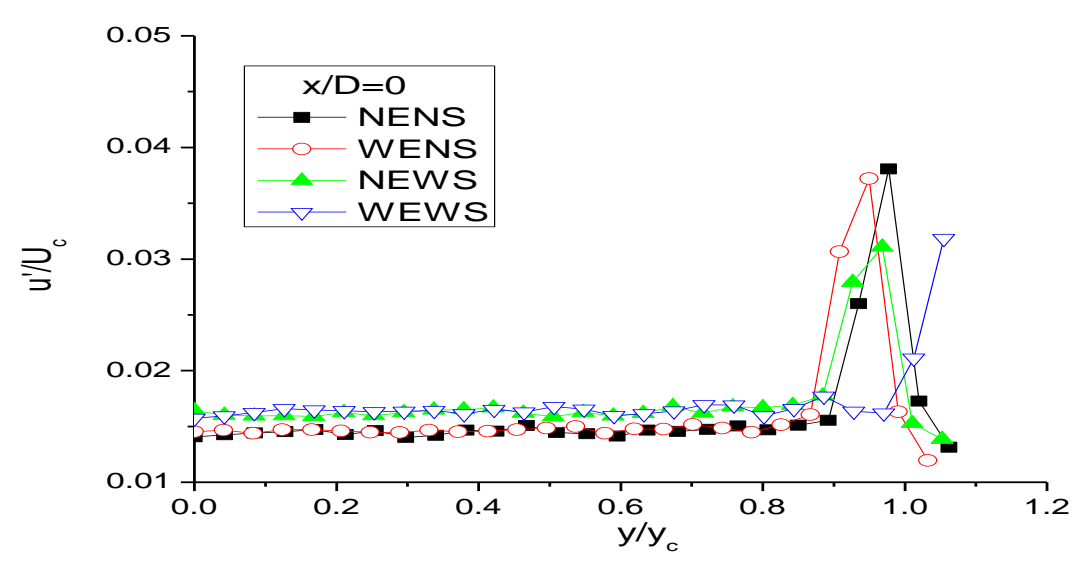

b)

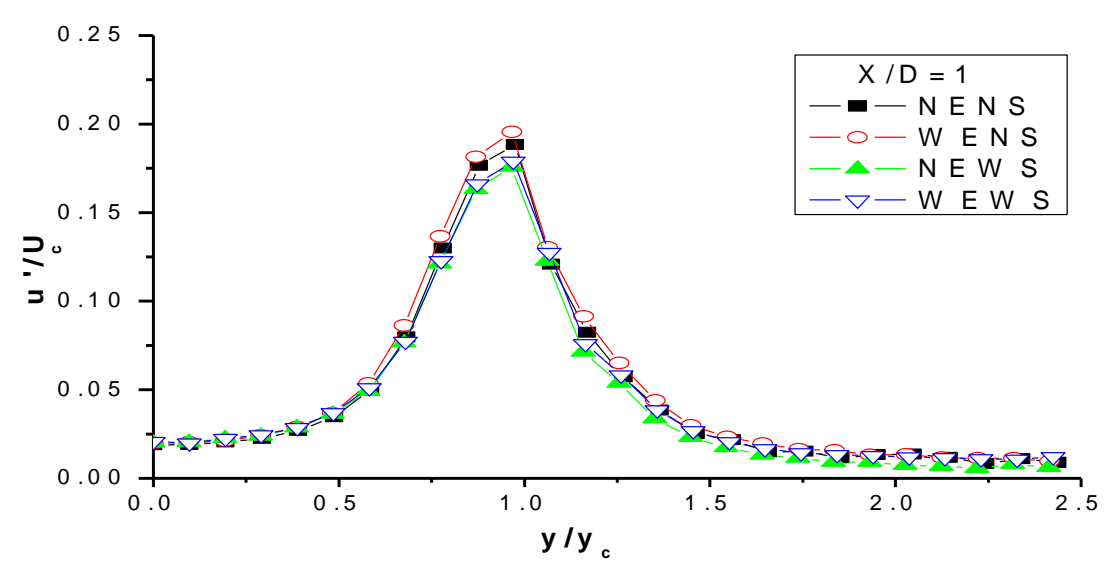

Figure 5.11: Transverse distributions of longitudinal turbulent velocities on the central $x y$ plane normalized to the centreline velocity at different downstream locations. 
c)

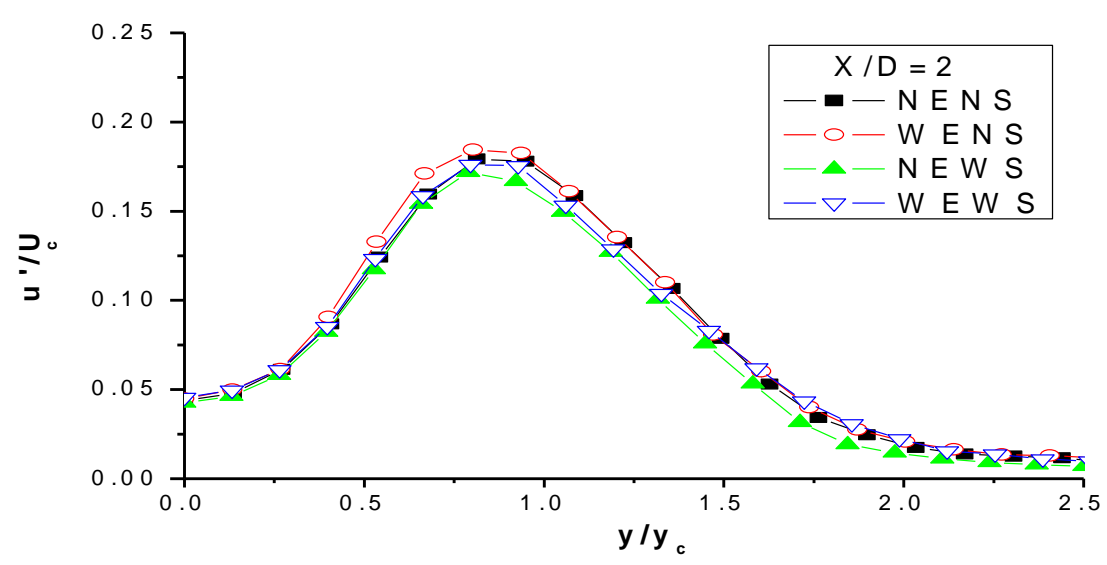

d)

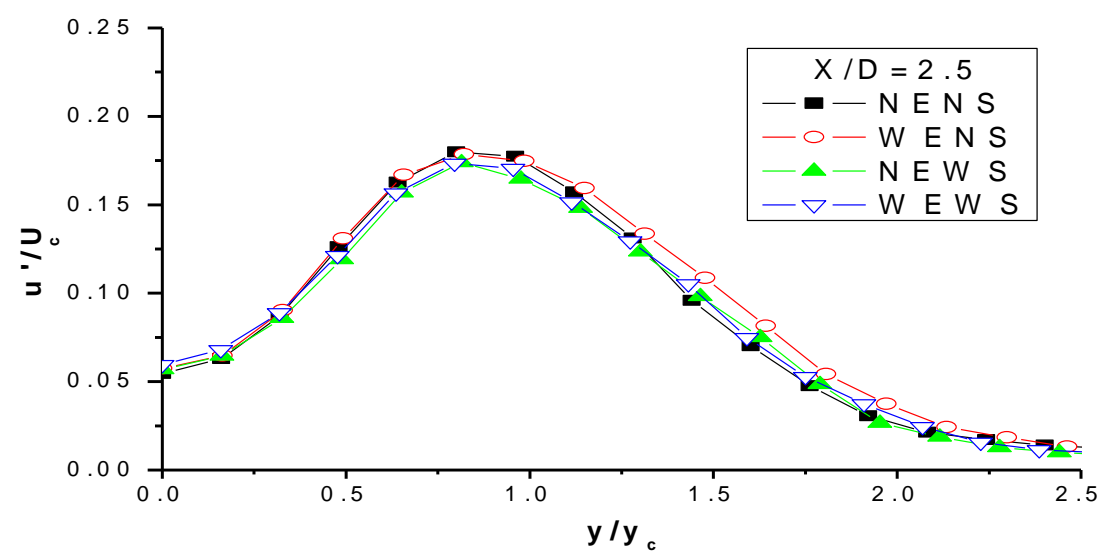

e)

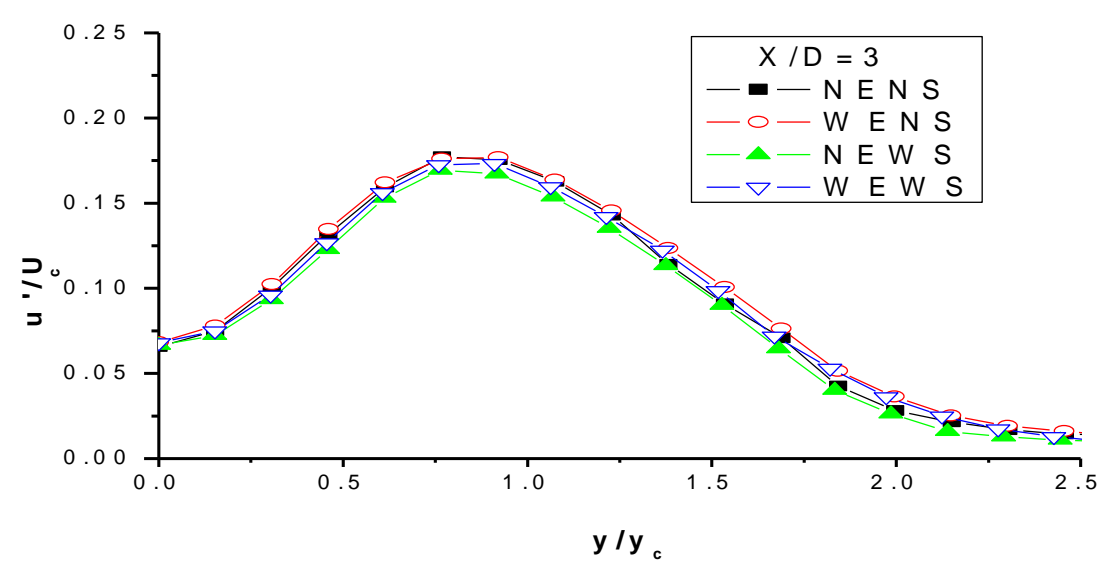

Figure 5.11: Transverse distributions of longitudinal turbulent velocities on the central $x y$ plane normalized to the centreline velocity at different downstream locations (cont'd). 
f)

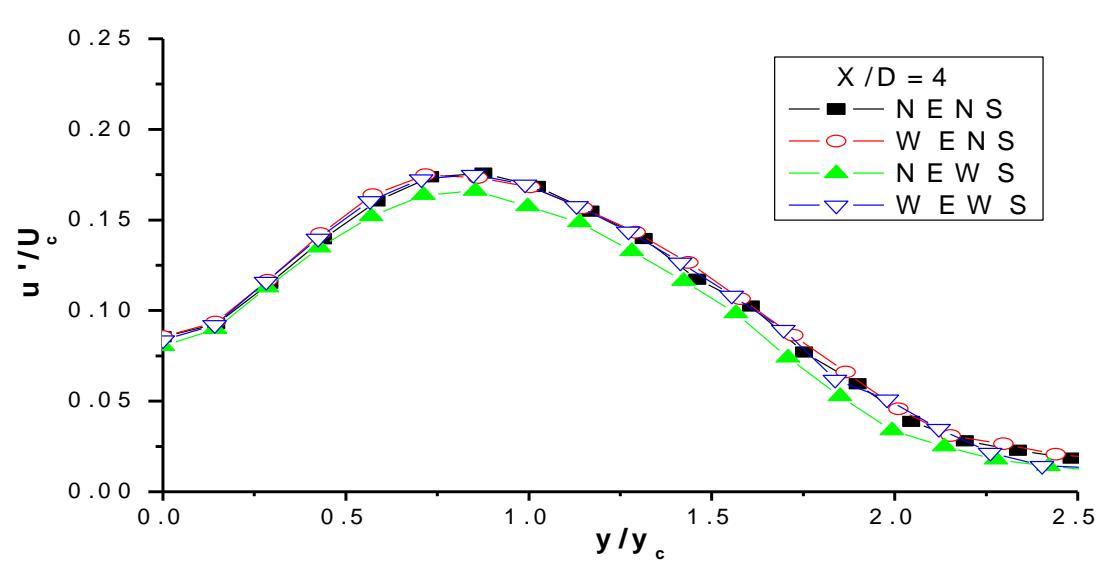

g)

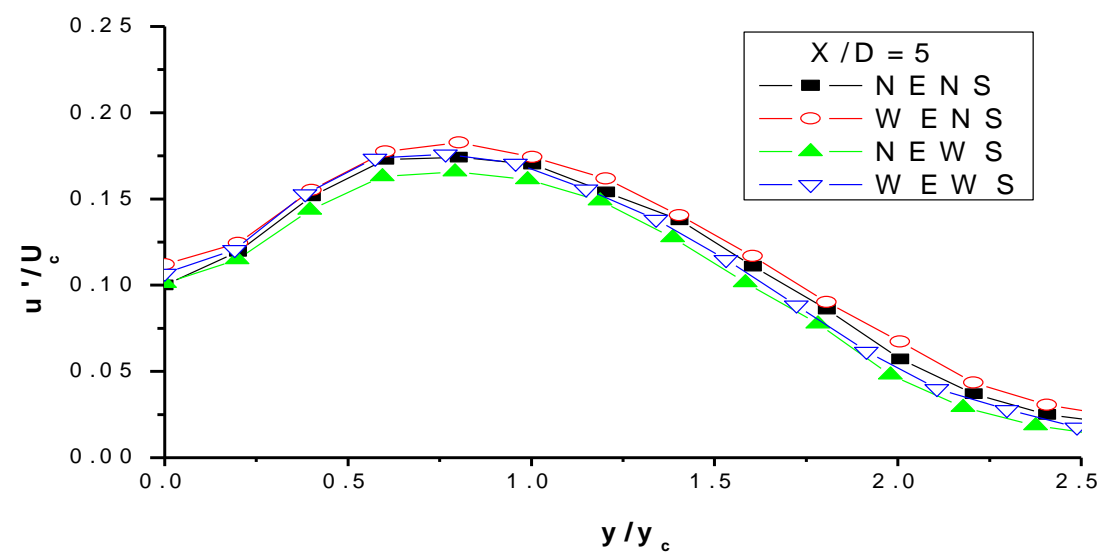

h)

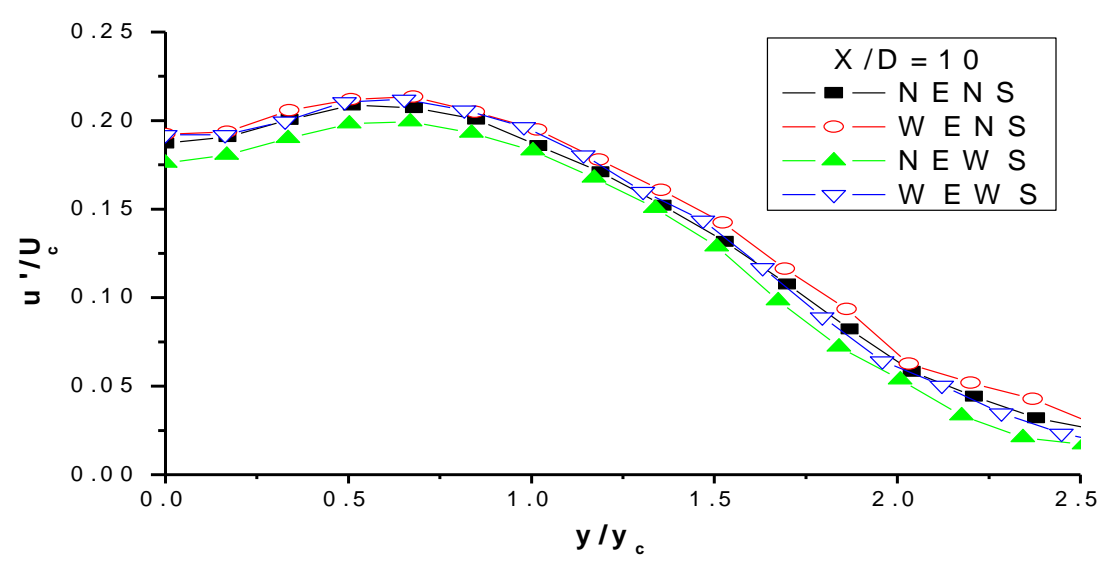

Figure 5.11: Transverse distributions of longitudinal turbulent velocities on the central $x y$ plane normalized to the centreline velocity at different downstream locations (cont'd). 
j)

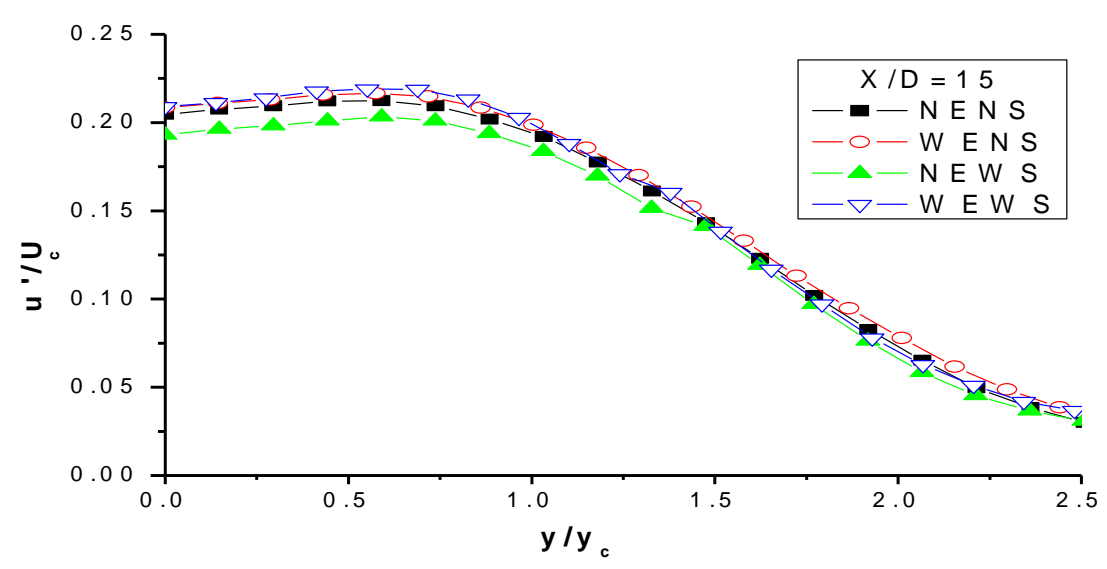

$\mathrm{k})$

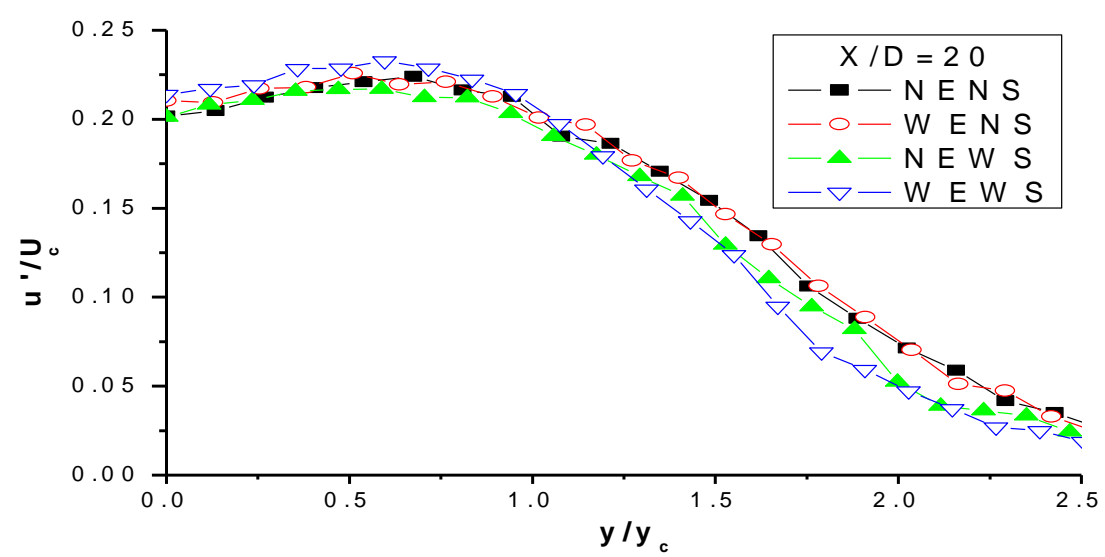

1)

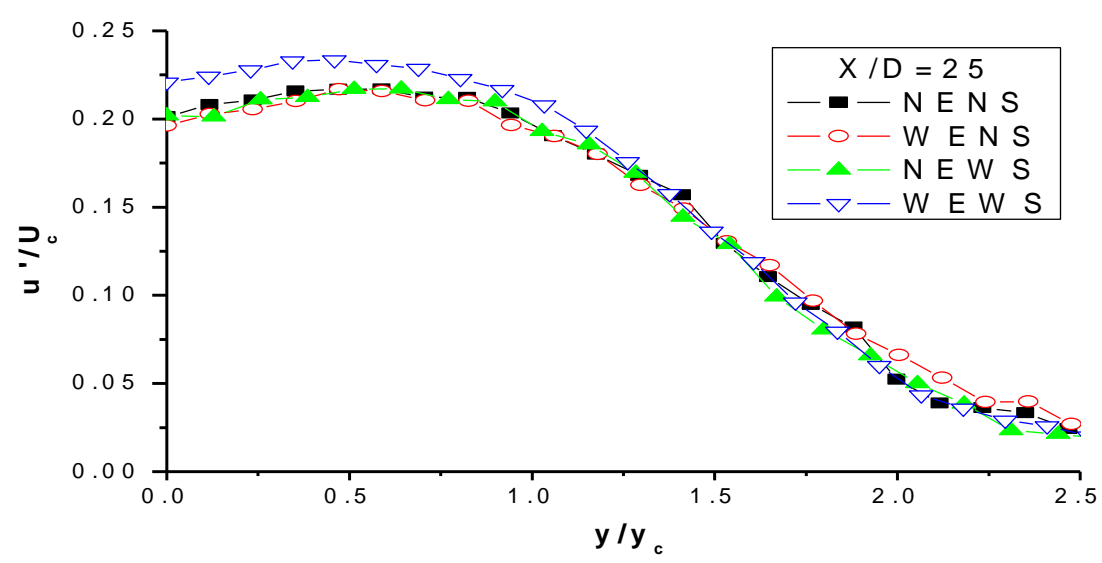

Figure 5.11: Transverse distributions of longitudinal turbulent velocities on the central $x y$ plane normalized to the centreline velocity at different downstream locations (cont'd). 
$\mathrm{m})$

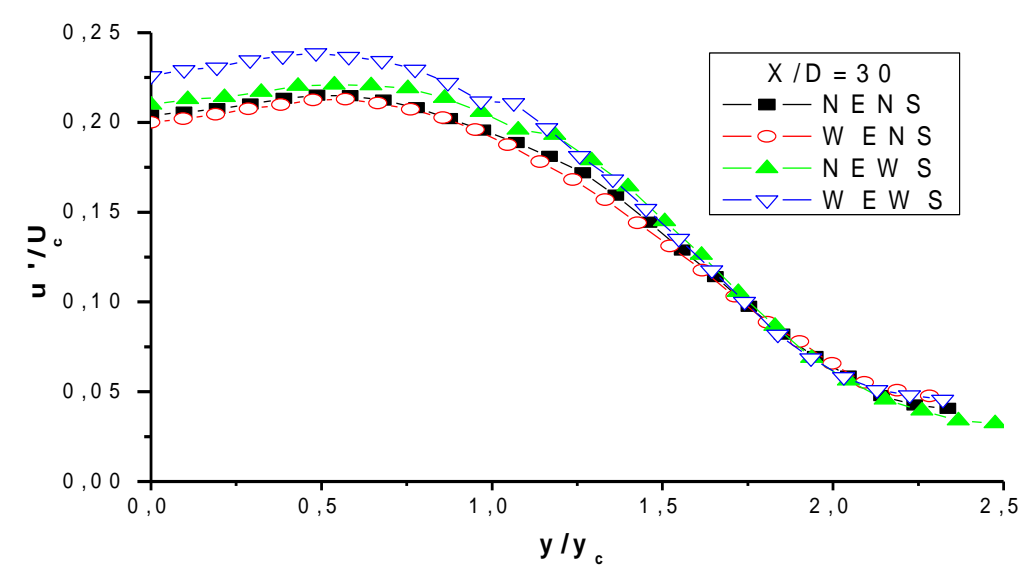

n)

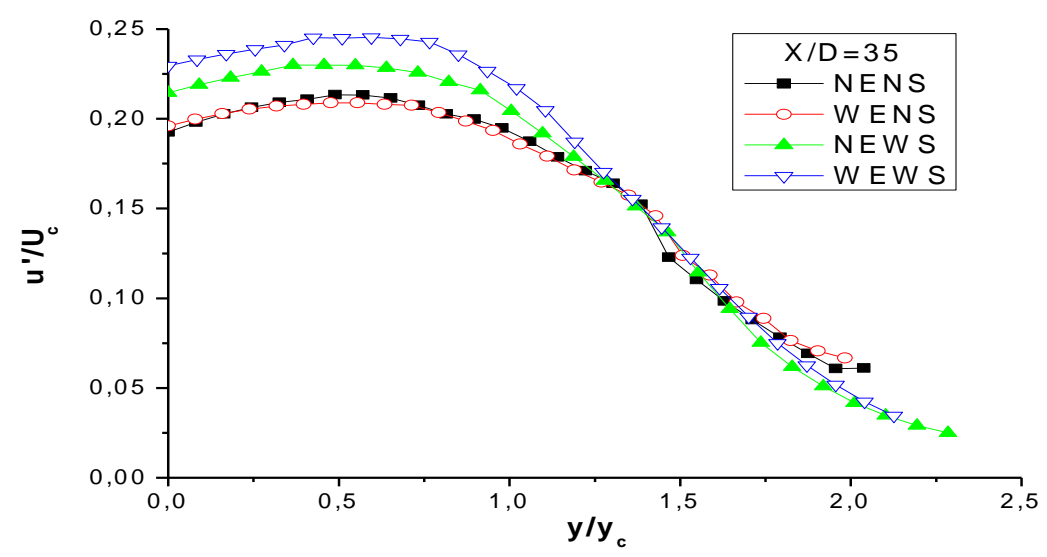

Figure 5.11: Transverse distributions of longitudinal turbulent velocities on the central $x y$ plane normalized to the centreline velocity at different downstream locations (cont'd).

\section{b) Similarity of longitudinal turbulent velocity profiles}

Figure 5.12 examines the relationship between the self-preserving behavior of the $u^{\prime}$ profiles and the presence and/or absence of the endplate and/or sidewalls. It is found that whereas the normalized $u^{\prime}$ profiles for the jets without sidewalls (NENS and WENS) at various distances from the exit in the range $x / D=15-35$ are almost indistinguishable (see figures 5.12-a and c), the profiles for the jets with sidewalls (NEWS, WEWS) indicate a monotonic increase of the values in the central area of the jet accompanied by a displacement of the peak values location towards the centreline as $x / D$ increases, preventing the $u^{\prime}$ profiles to attain similarity (Figures 5.12-b and d). 
a)

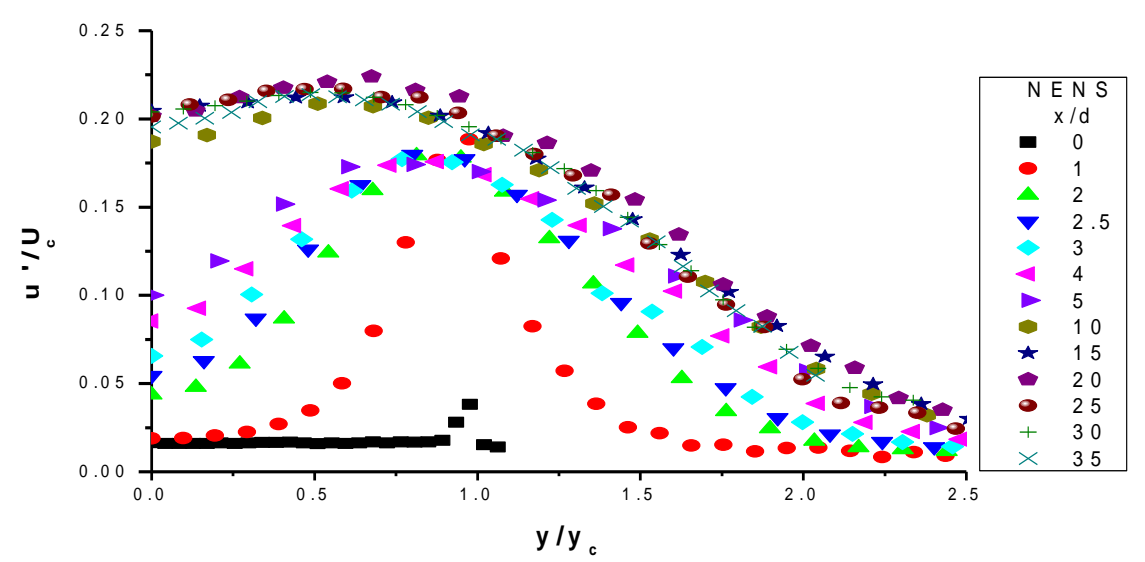

b)

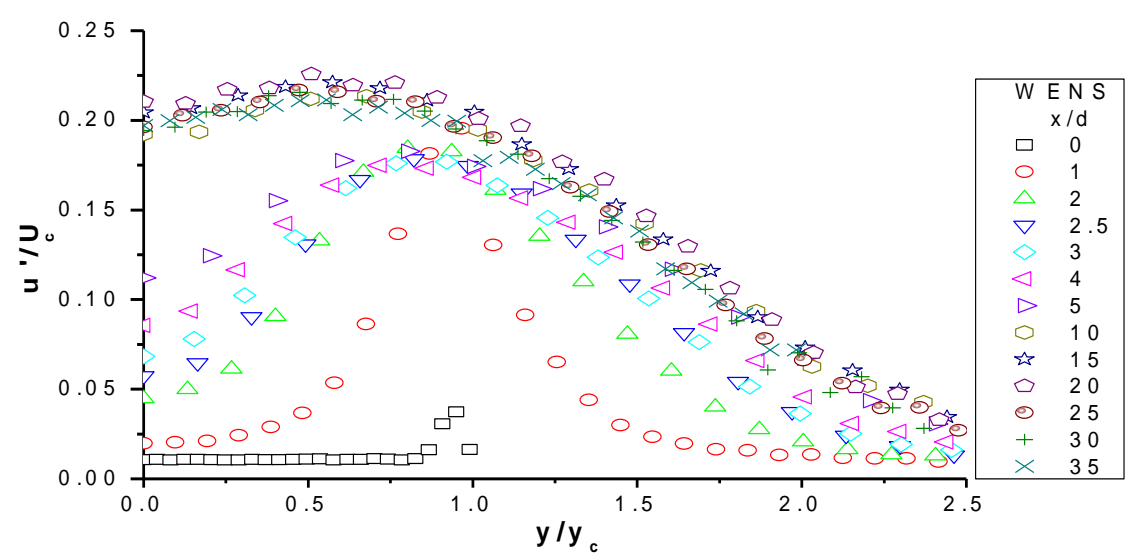

c)

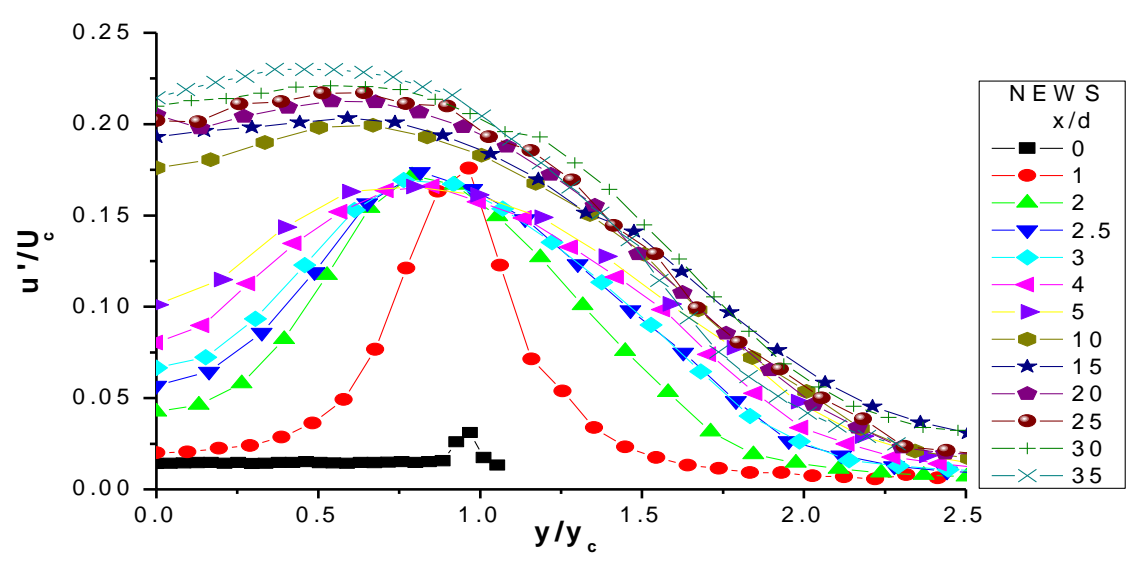

Figure 5.12: Self similarity of transverse distributions of longitudinal turbulent velocities on the central $x y$ plane for different configurations: a) NENS, b) WENS, c) NEWS, and d) WEWS. 
d)

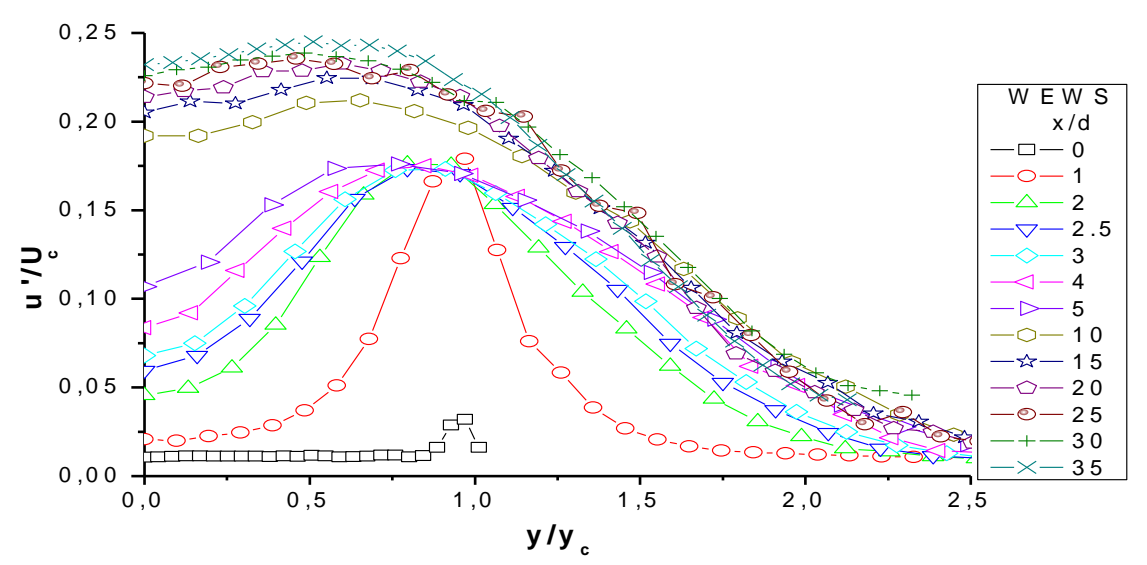

Figure 5.12: Self similarity of transverse distributions of longitudinal turbulent velocities on the central $x y$ plane for different configurations: a) NENS, b) WENS, c) NEWS, and d) WEWS (cont'd).

Published results on two-dimensional jets have shown variations in the downstream location where the turbulent velocity profiles (e.g. $u^{\prime} / U_{c}$ ) collapse on a single curve (see table 5.2). The present results suggest that the presence of the sidewalls or both sidewalls and endplate can influence the turbulent velocity components beside other various initial and boundary condition (aspect ratio, e.g. Deo et al., 2007a, nozzle

\begin{tabular}{|c|c|c|c|c|c|}
\hline Source & $\begin{array}{c}\text { Conditio } \\
\mathbf{n}\end{array}$ & $\begin{array}{l}\text { Aspec } \\
\text { t ratio }\end{array}$ & $\begin{array}{l}\text { Reynolds } \\
\text { number }\end{array}$ & Range & $\begin{array}{c}\text { Self- } \\
\text { preservation, } \\
x / D \\
\end{array}$ \\
\hline Present investigation & $\begin{array}{l}\text { NENS } \\
\text { WENS } \\
\text { NEWS } \\
\text { WEWS }\end{array}$ & 15 & $20 \times 10^{3}$ & $x / D \leq 35$ & $\begin{array}{c}15\left(u^{\prime}\right) \\
15\left(u^{\prime}\right) \\
* \\
*\end{array}$ \\
\hline $\begin{array}{l}\text { Gutmark \& Wygnanski, } \\
\text { (1976) }\end{array}$ & WEWS & 38 & $30 \times 10^{3}$ & $x / D \leq 120$ & $40 * *$ \\
\hline Evritt \& Robins, (1978) & NEWS & $\begin{array}{c}128 \\
64 \\
32 \\
21\end{array}$ & $\begin{array}{l}16 \times 10^{3} \\
30 \times 10^{3} \\
30 \times 10^{3} \\
75 \times 10^{3}\end{array}$ & & $\begin{array}{l}70-100\left(u^{\prime}\right) \\
40-60\left(u^{\prime}\right) \\
20-35\left(u^{\prime}\right) \\
20-30\left(u^{\prime}\right)\end{array}$ \\
\hline Krothapalli et al., (1981) & NENS & 16 & $12 \times 10^{3}$ & $x / D \leq 115$ & $30(u v)$ \\
\hline
\end{tabular}

*Self-preservation not attained, ** based on the turbulent velocities. 


\section{c) Lateral distributions of the longitudinal turbulent velocities normalized to the exit velocity}

To identify the effect of the different decay rates to the turbulent velocities already discussed some measurements presented in Figures 5.11 normalised by $U_{c}$ are repeated in Figures 5.13-1, b, c and d normalised by $U_{0}$ for downstream locations $x / D=1,4,15$ and 30 respectively. It is interesting to note that in these graphs the discussed differences are minimised and in most cases the curves can be distinguished on the basis of the presence or absence of sidewalls. In the early stages of development, the measurements are almost identical for the configurations without sidewalls, whereas when the sidewalls are present the values are generally lower with those corresponding to the absence of an endplate being the lowest. At the furthest location, all measurements almost collapse to a single curve although a trend indicating that the values in the presence of sidewalls are becoming larger may be identified in the $u^{\prime}$ measurements.

a)

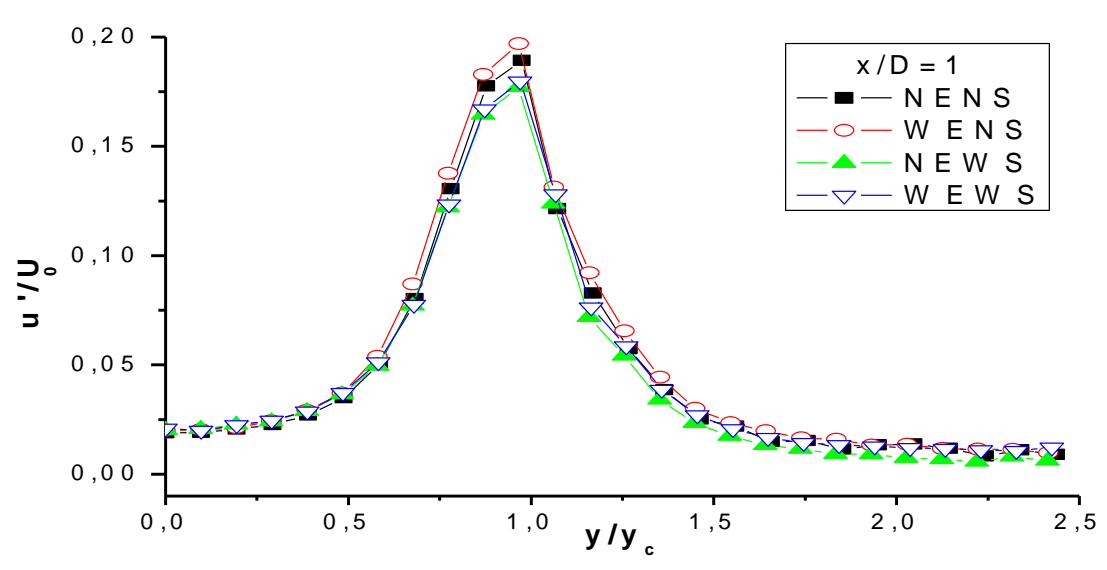

Figure 5.13: Turbulent intensity distributions of the streamwise velocity component on the central $x y$ plane normalised to the exit velocity at different downstream locations. 
b)

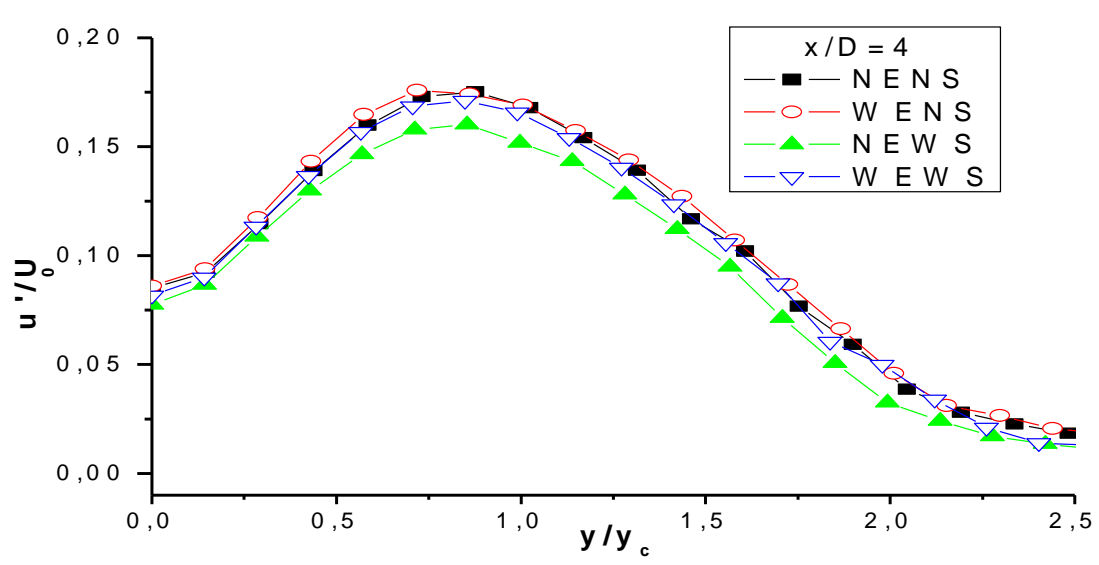

c)

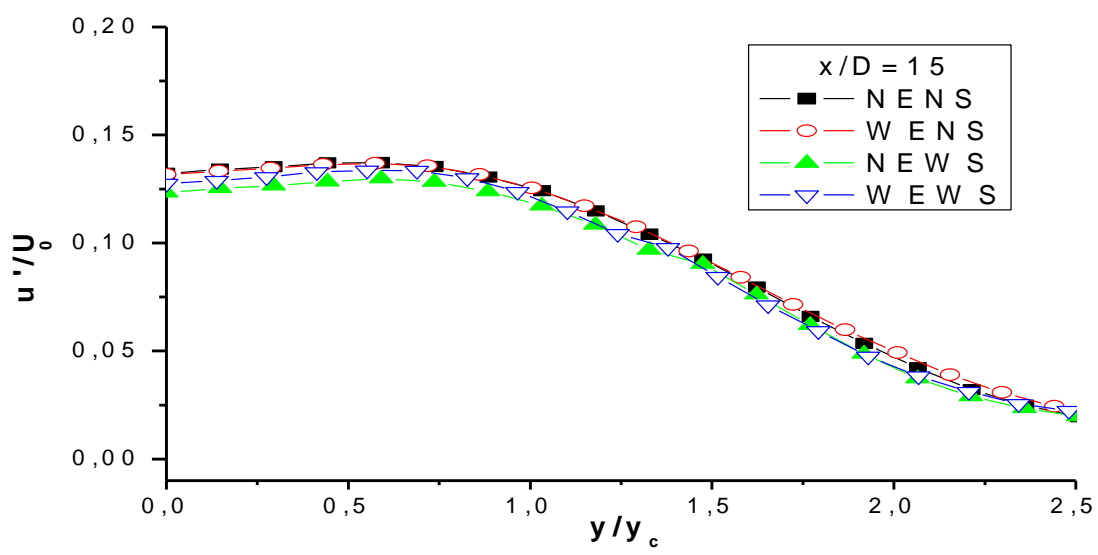

d)

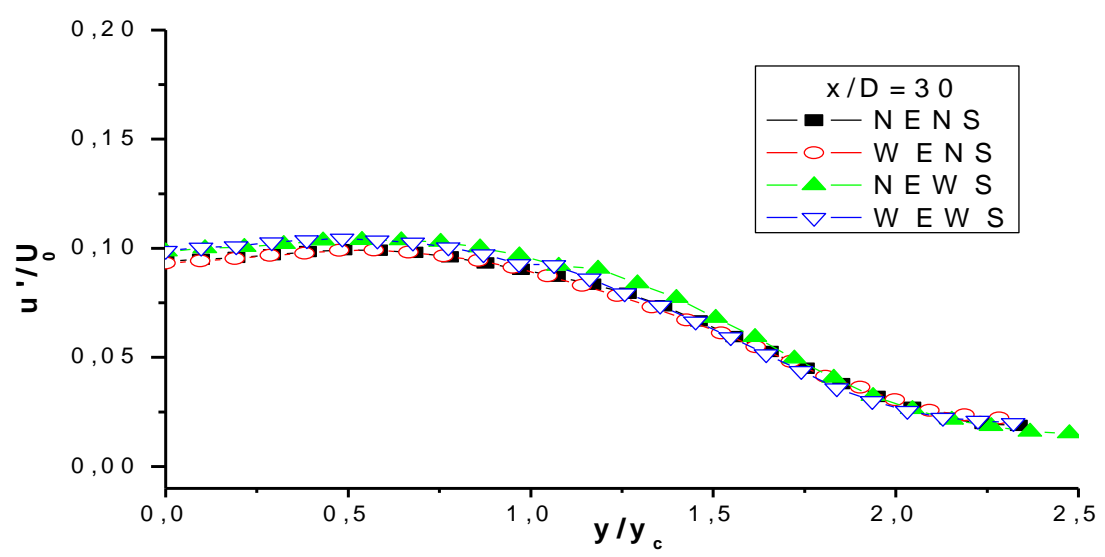

Figure 5.13: Turbulent intensity distributions of the streamwise velocity component on the central $x y$ plane normalised to the exit velocity at different downstream locations (cont'd). 


\section{d) Evolution of the centreline longitudinal turbulent velocity at various Re}

To further investigate the development of $u^{\prime}$ at various Reynolds numbers, centreline variations of the longitudinal turbulent velocity, $u^{\prime}$ normalized by the centreline mean streamwise velocity at $R e=10,000,20,000$ and 30,000 for configurations NENS, WENS, NEWS, and WEWS are presented in Figures 5.14-a, b, and c respectively. The shape of the development of $u^{\prime}$ at various Reynolds numbers for all configurations is in agreement with the available distributions in the literature. The monotonic increase of longitudinal turbulent velocity, which has been observed in the far field's transverse profiles (NEWS and WEWS, Figures 5.12-c and d respectively), is verified by the centreline distributions (Figures 5.14-b). From these figures one may conclude that the presence of endplate and sidewalls lead to profound effects on the development of the streamwise turbulent velocity. These effects vary with varying Reynolds number.

a)

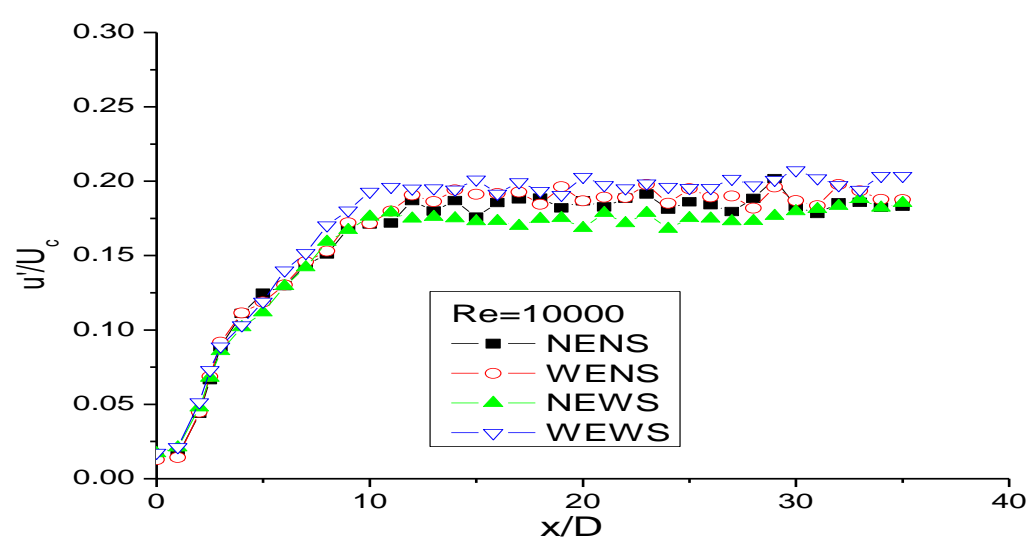

Figure 5.14: Centreline distributions of longitudinal turbulent velocity for all configurations at Reynolds numbers: a) 10,000, b) 20,000 and c) 30,000. 
b)

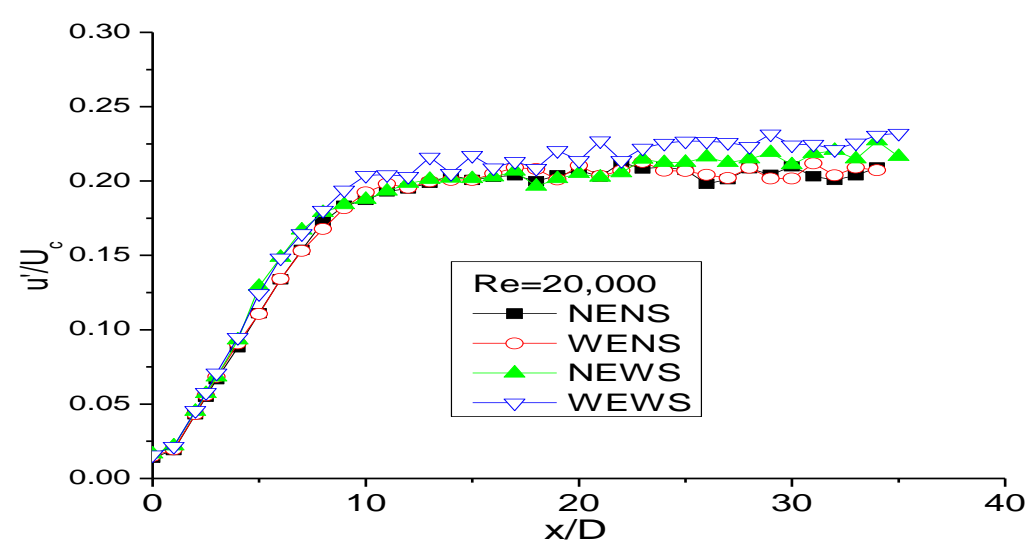

c)

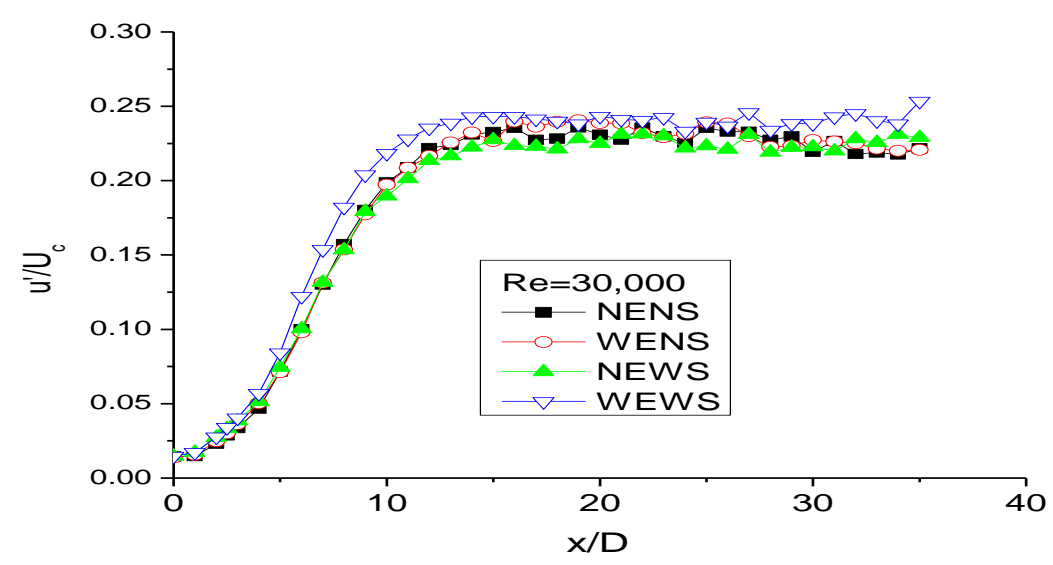

Figure 5.14: Centreline distributions of longitudinal turbulent velocity for all configurations at Reynolds numbers: a) 10,000, b) 20,000 and c) 30,000 (con't).

To study the effect of Reynolds number on $u^{\prime}$ for each configuration, the data presented in Figures 5.14-a, b and $\mathrm{c}$ are rearranged based on configuration and presented in Figures 5.15-a, b, c and d. The influence of $R e$ for most configurations is similar except for the cases that have sidewalls and endplate, are associated with the previously discussed monotonic increase.

As shown in Figures 5.15-a, b, c and d, the rapid increase in the magnitude of $u^{\prime}$ is observed in the near field for all configurations. Further downstream beyond $x / D=10$, the magnitude of $u^{\prime}$ is found to be again related to $R e$ having different trend. As $R e$ increases the magnitude of $u^{\prime}$ also increases. Deo et al., (2008) have found the same trends when they investigated the influence of Reynolds numbers in the range between 1,500 and 16,500. Their magnitude of $u^{\prime}$ was also increasing with Reynolds number beyond $x / D=13$. 
Deo et al. (2008) have studied the relationship between Reynolds number and vortex shedding. They found that with the increase of Reynolds number from 1,500 to 16,500 , the vortex passage frequency (or Strouhul number, St) was slightly increased from 0.201 to 0.218 indicating weak dependence. The same team, estimated the St for two jets one with sidewalls and the other without (NENS, NEWS) and found that the St was 0.22 and 0.36 for jet with sidewalls (NEWS) and free jet (NENS) respectively. Their findings implied that Reynolds number and nozzle condition affect the vortex formation and its interaction. Although we have not estimated the St, we may suspect that the variations among the configurations at various Re are due to the differences in the vortex formation and its interaction.

a)

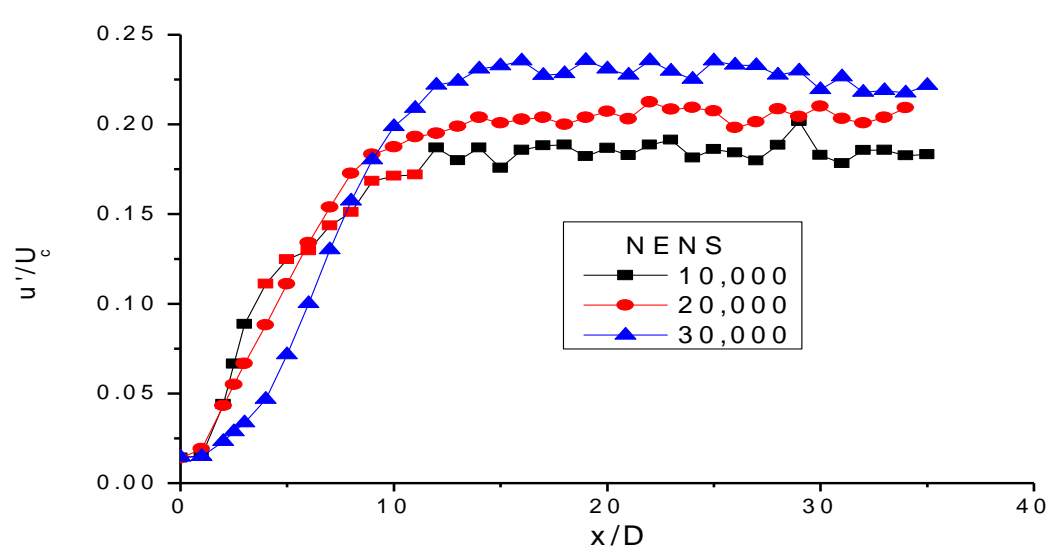

Figure 5.15: Reynolds number $R e$ dependence of the centerline longitudinal turbulent velocity for: a) NENS, b) WENS, c) NEWS and d) WEWS. 
b)

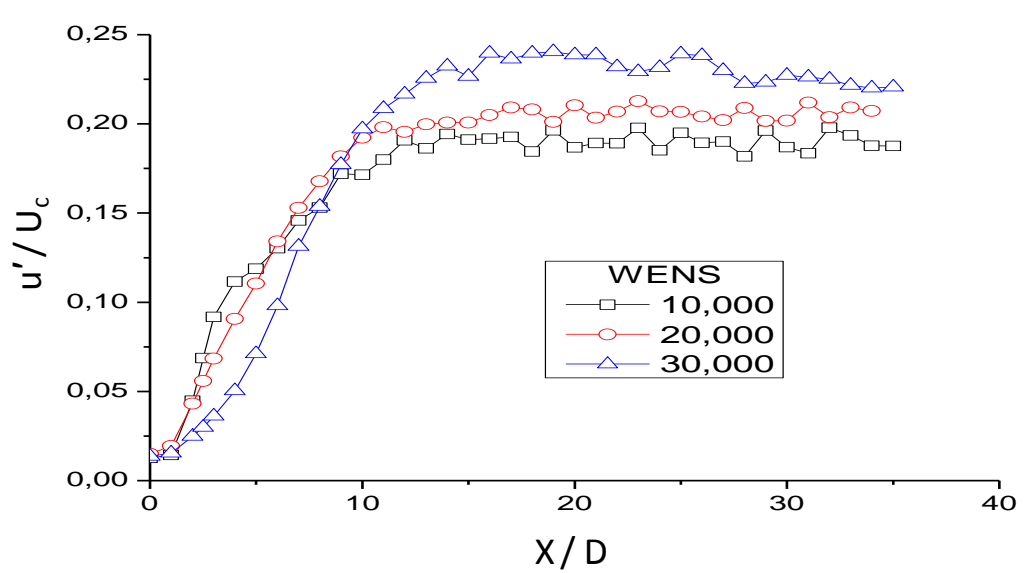

c)

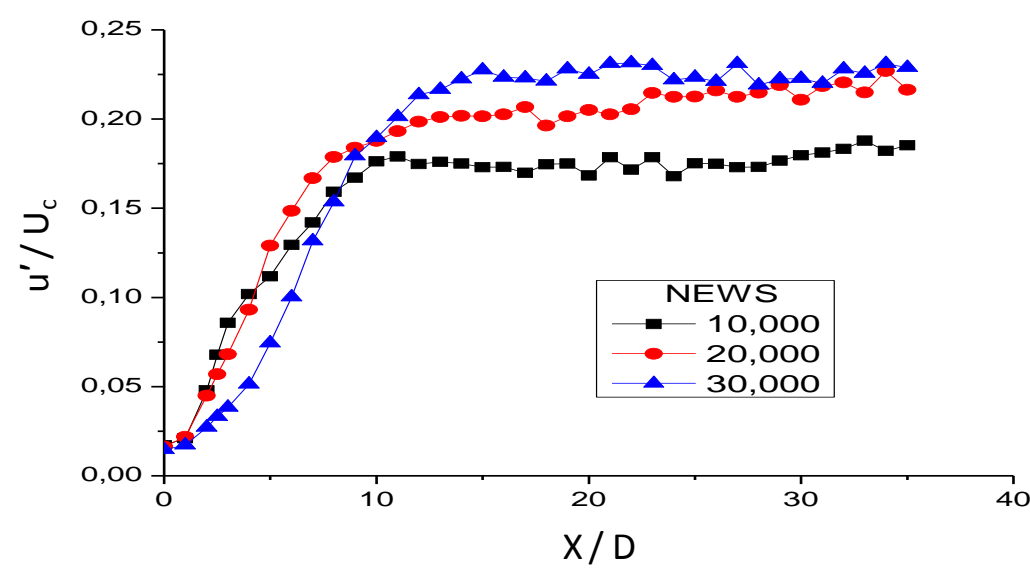

d)

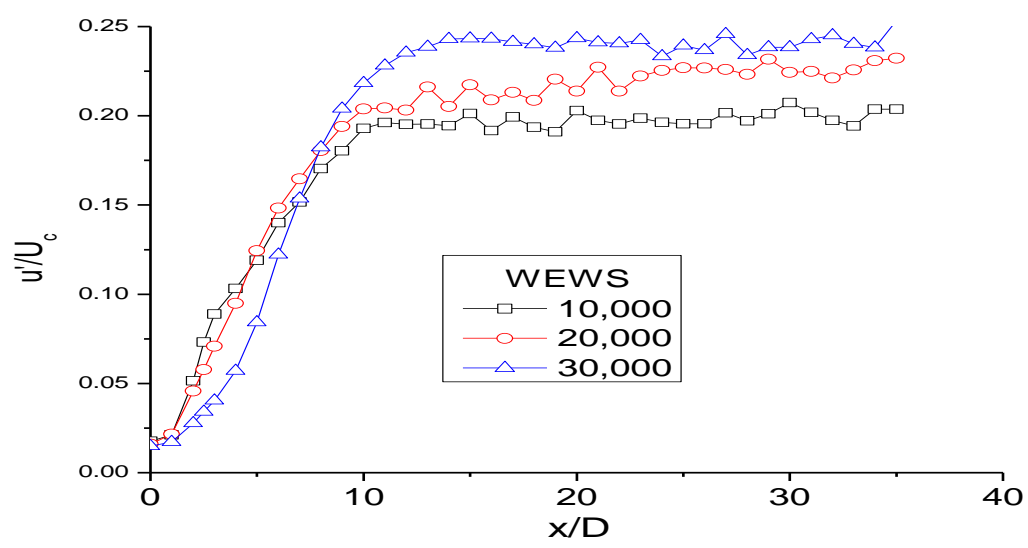

Figure 5.15: Reynolds number $R e$ dependence of the centerline longitudinal turbulent velocity for: a) NENS, b) WENS, c) NEWS and d) WEWS (con’t). 


\subsubsection{Lateral turbulent velocity}

a) Transverse distributions of lateral turbulent velocities normalized to the centreline velocity

The lateral distributions of the lateral turbulent velocity, $v^{\prime}$, on the central $x y$-plane, for all four configurations at $R e=20,000$, are shown in figures 5.14-a, b, c, d, e, f, g, h, $\mathrm{j}, \mathrm{k}, 1, \mathrm{~m}$ and $\mathrm{n}$ at $x / D=0,1,2,2.5,3,4,5,10,15,20,25,30$, and 35 respectively.

The distributions of the four configurations have the expected shapes which follow in general the trends observed in turbulent longitudinal velocity. And the magnitudes of $v^{\prime}$ are smaller than the corresponding $u^{\prime}$.

a)

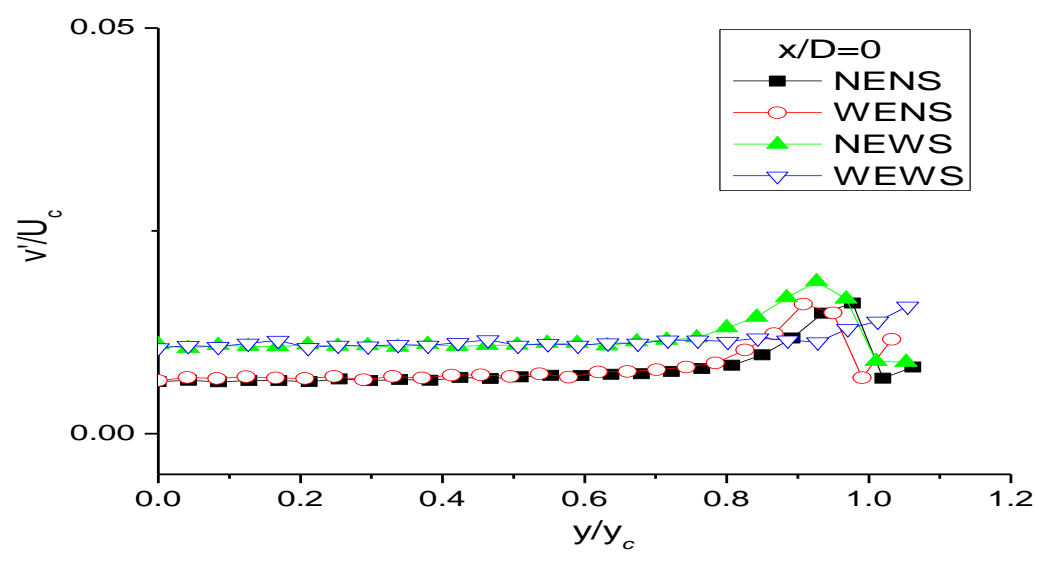

b)

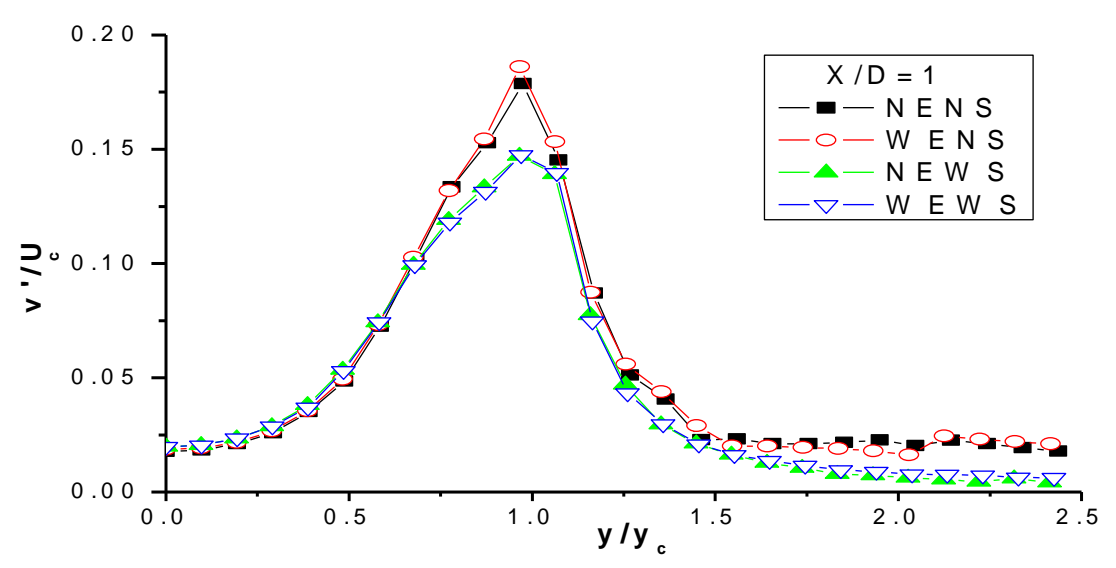

Figure 5.16: Turbulent intensity distributions of the lateral velocity component on the central $x y$ plane normalised to the centreline velocity at different downstream locations. 
c)

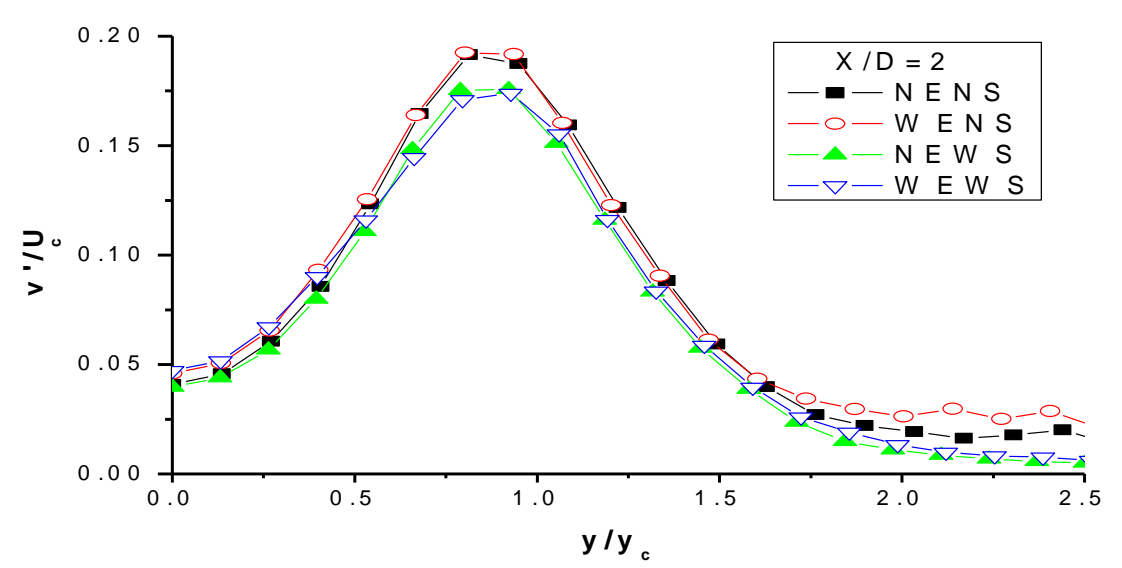

d)

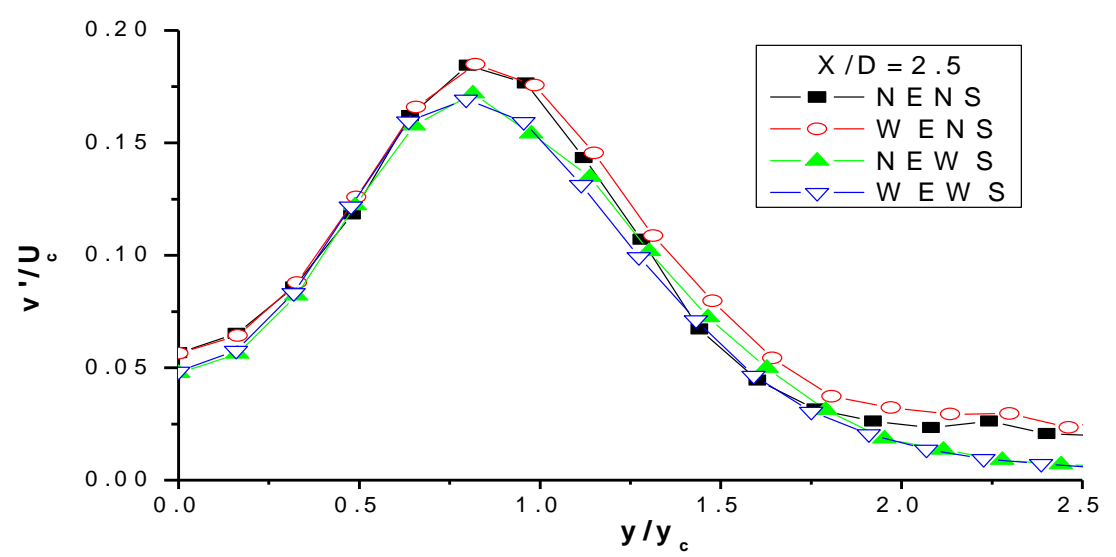

e)

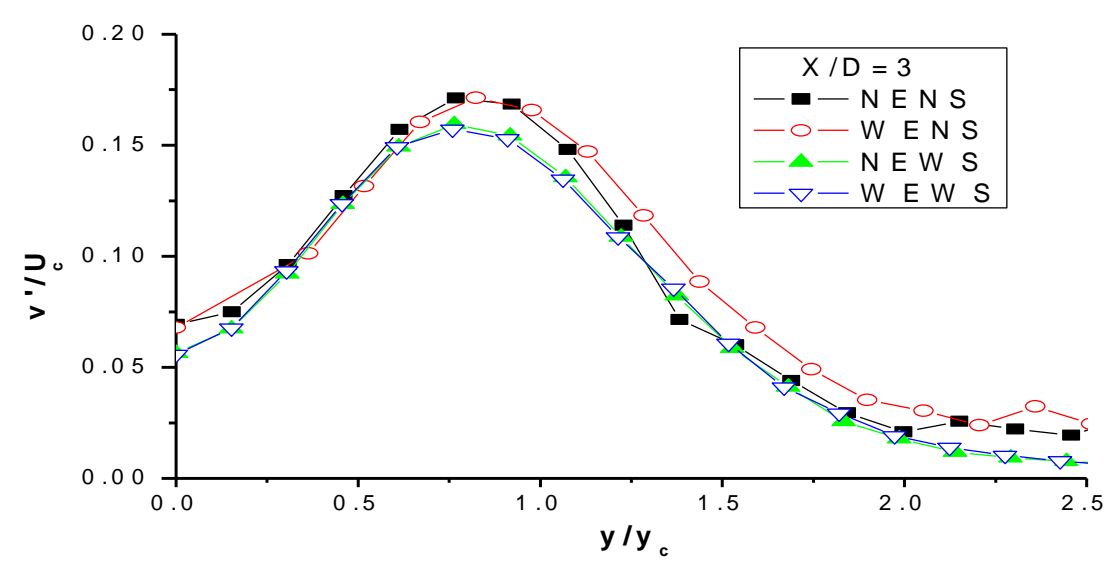

Figure 5.16: Turbulent intensity distributions of the lateral velocity component on the central $x y$ plane normalised to the centreline velocity at different downstream locations (cont'd). 
f)

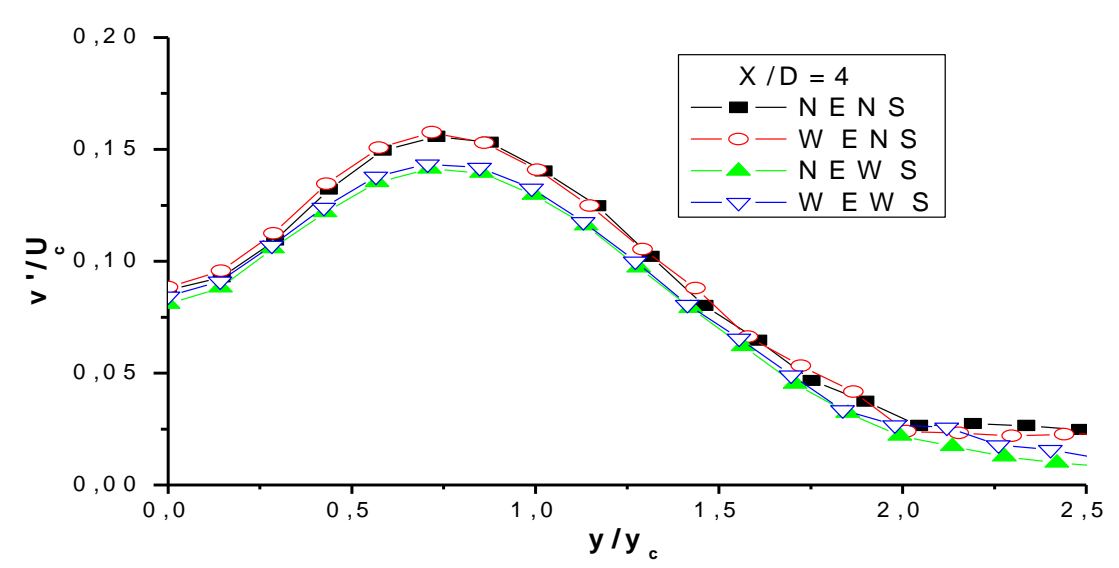

g)

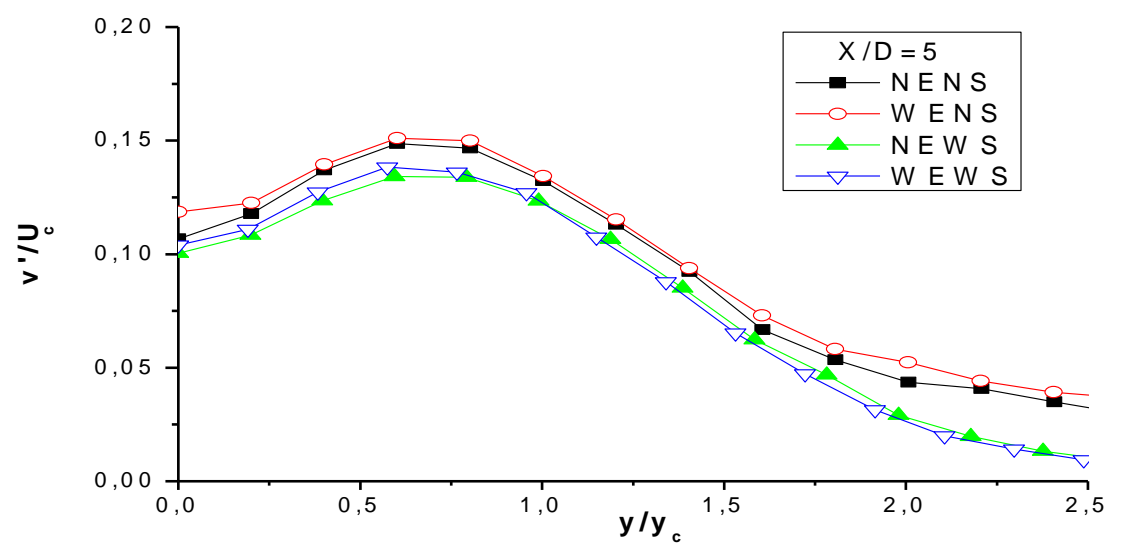

h)

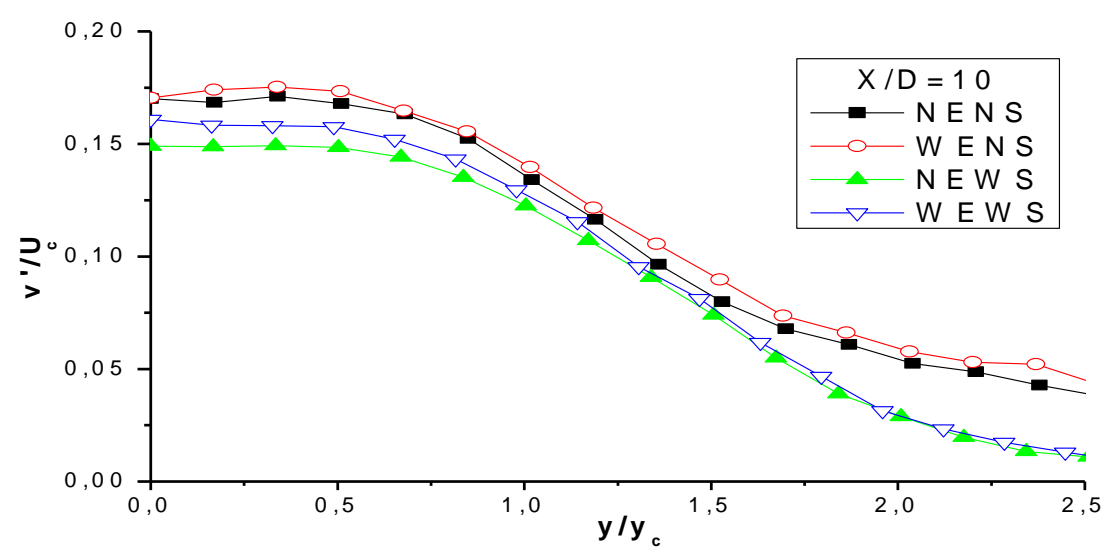

Figure 5.16: Turbulent intensity distributions of the lateral velocity component on the central $x y$ plane normalised to the centreline velocity at different downstream locations (cont'd). 
j)

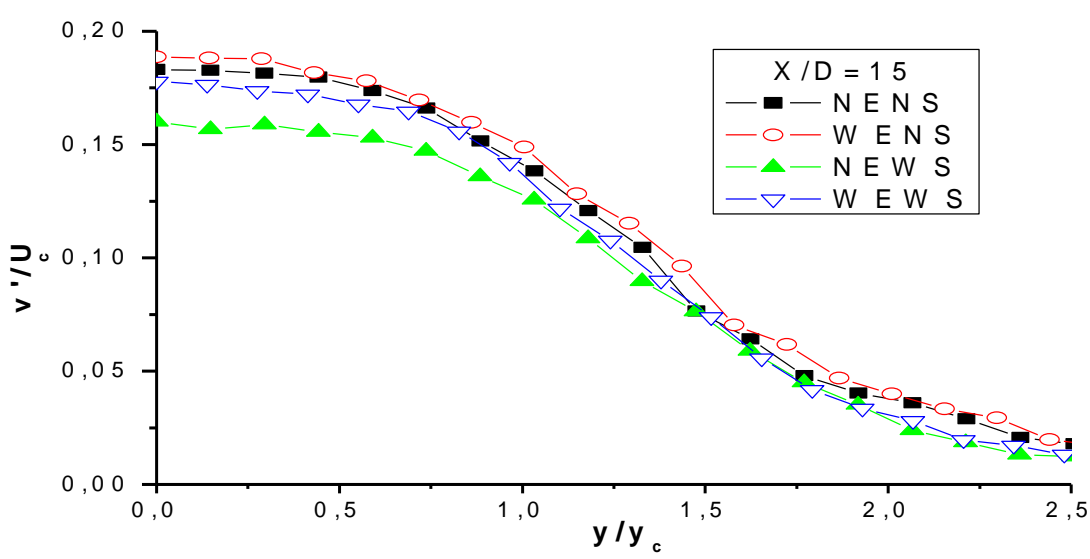

k)

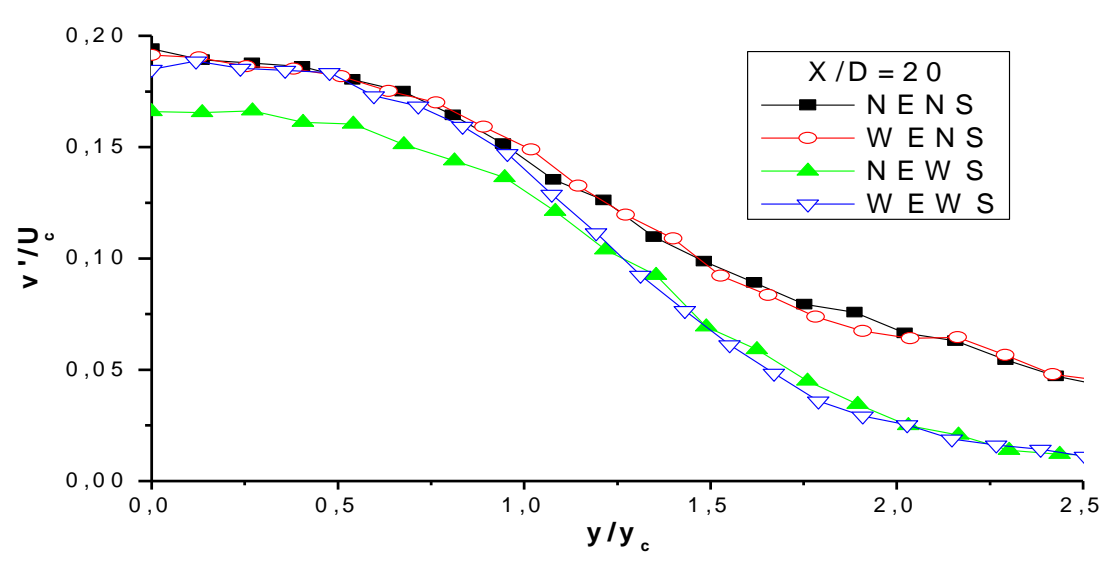

1)

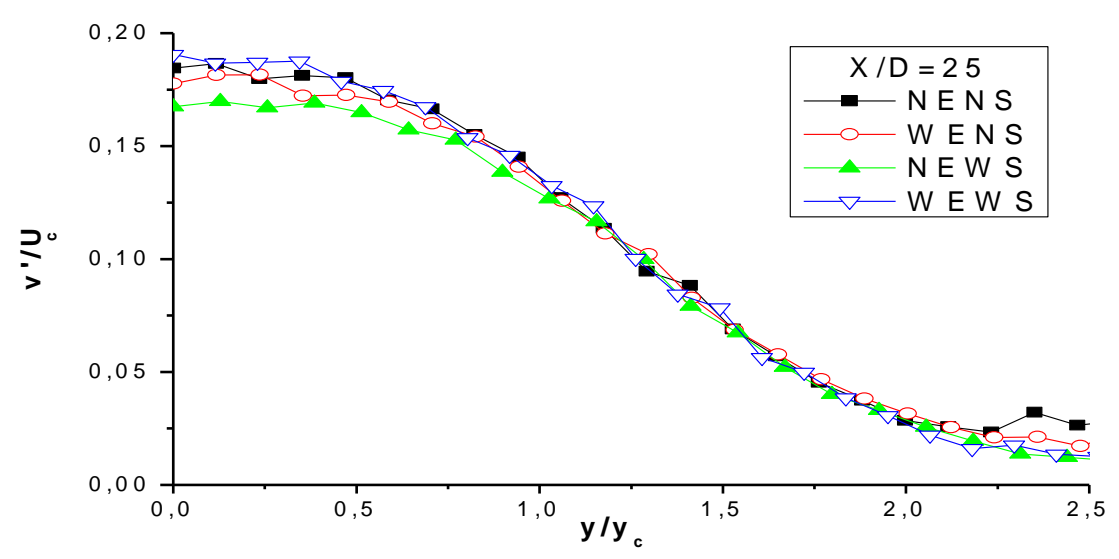

Figure 5.16: Turbulent intensity distributions of the lateral velocity component on the central $x y$ plane normalised to the centreline velocity at different downstream locations (cont'd). 
m)

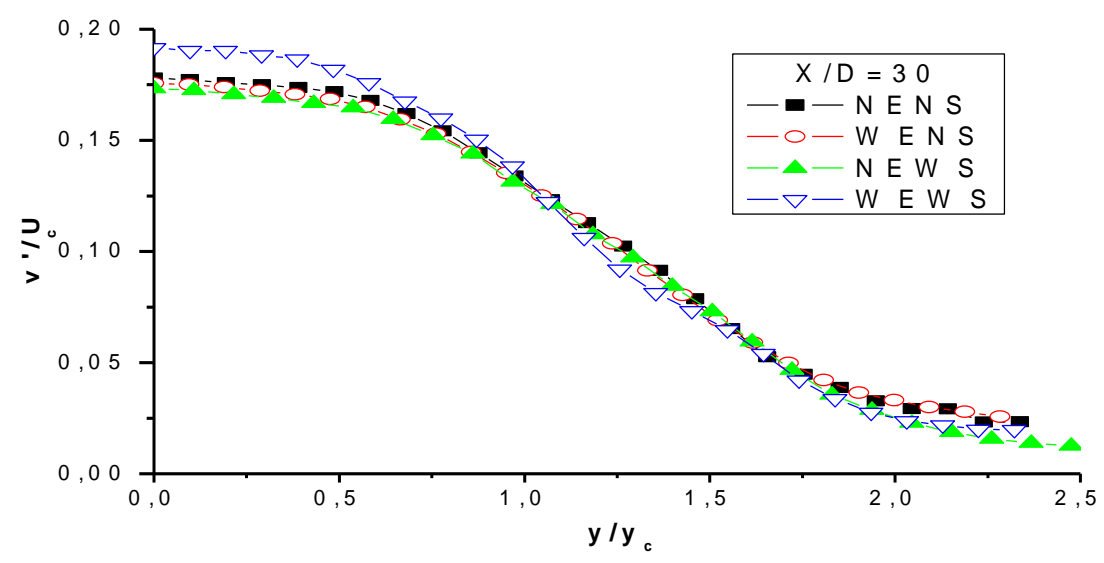

n)

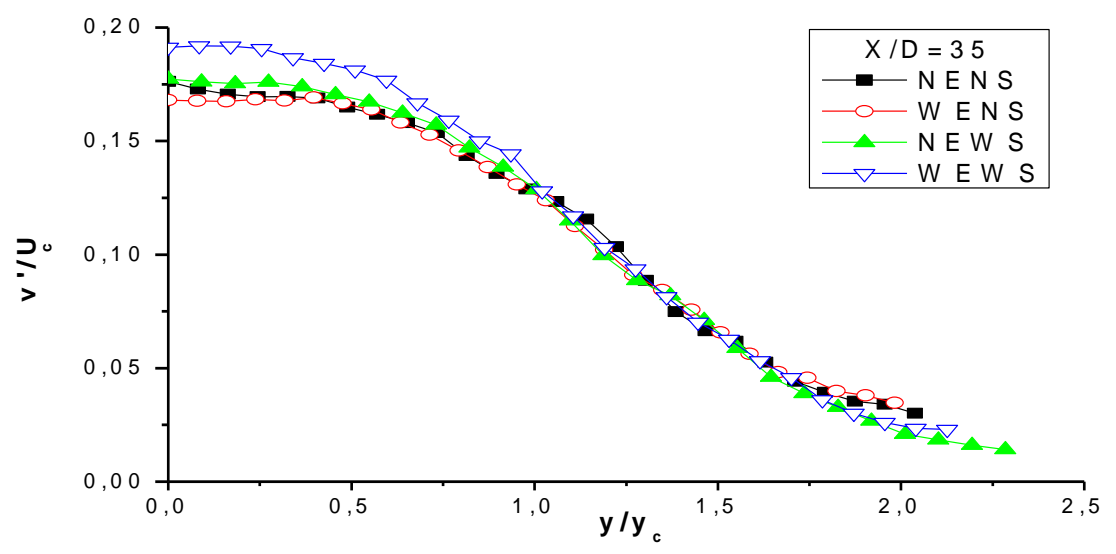

Figure 5.16: Turbulent intensity distributions of the lateral velocity component on the central $x y$ plane normalised to the centreline velocity at different downstream locations (cont'd).

\section{b) Similarity of lateral turbulent velocity profiles}

The self similarity behaviour of the normalized $v^{\prime}$ for NENS, WENS, NEWS and WEWS are shown in Figures 5.15a, b, c and d respectively. Although the profiles of $u^{\prime}$ in the absence of the sidewalls (NENS, WENS) became self-similar at about 15 nozzle width, the profiles of $v^{\prime}$ of the same test cases have not reached the self-similar behaviour. Similar trends hold true for the cases NEWS and WEWS. 
a)

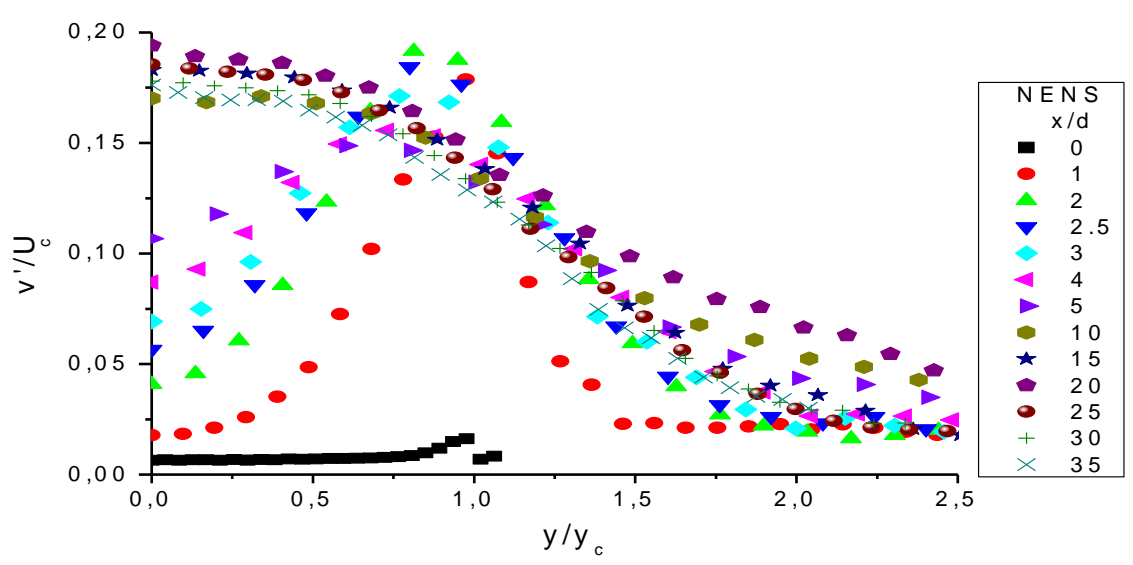

b)

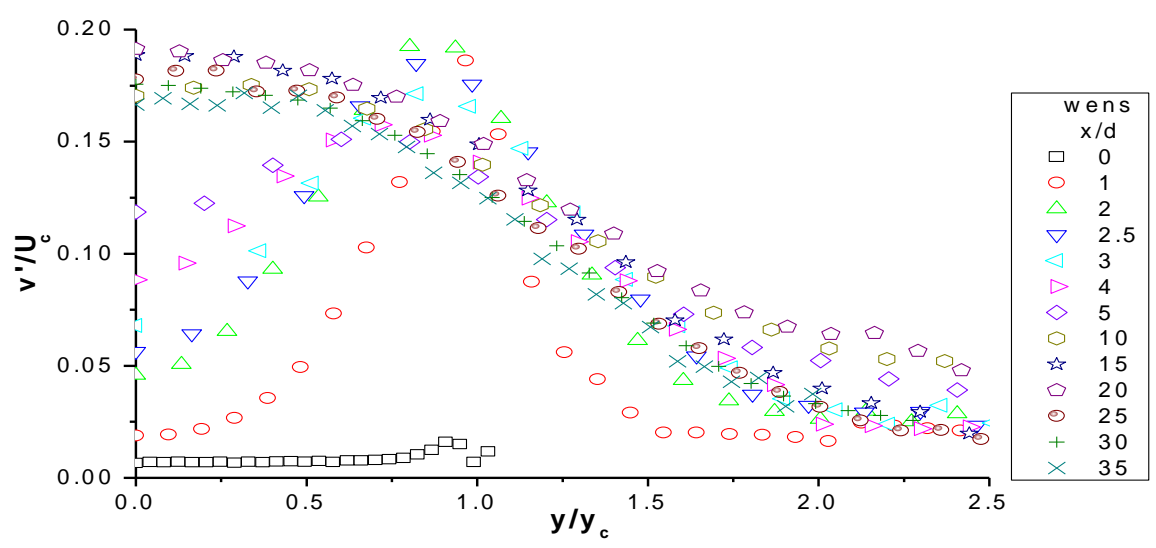

c)

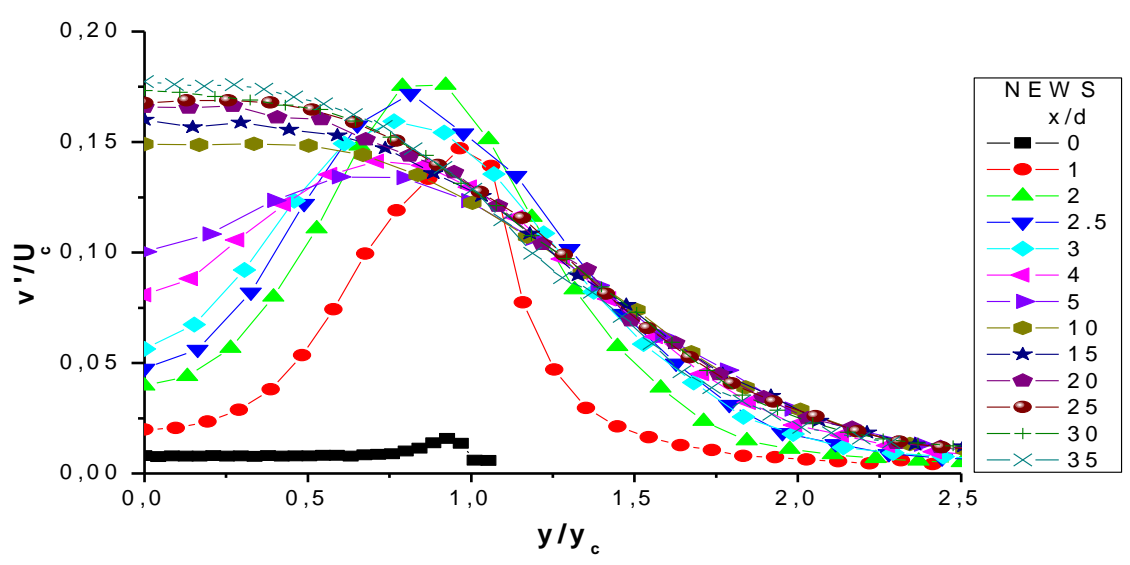

Figure 5.17: Self similarity of turbulent intensity distributions of the lateral velocity component on the central $x y$ plane for different test cases: a) NENS, b) WENS, c) NEWS and d) WEWS. 
d)

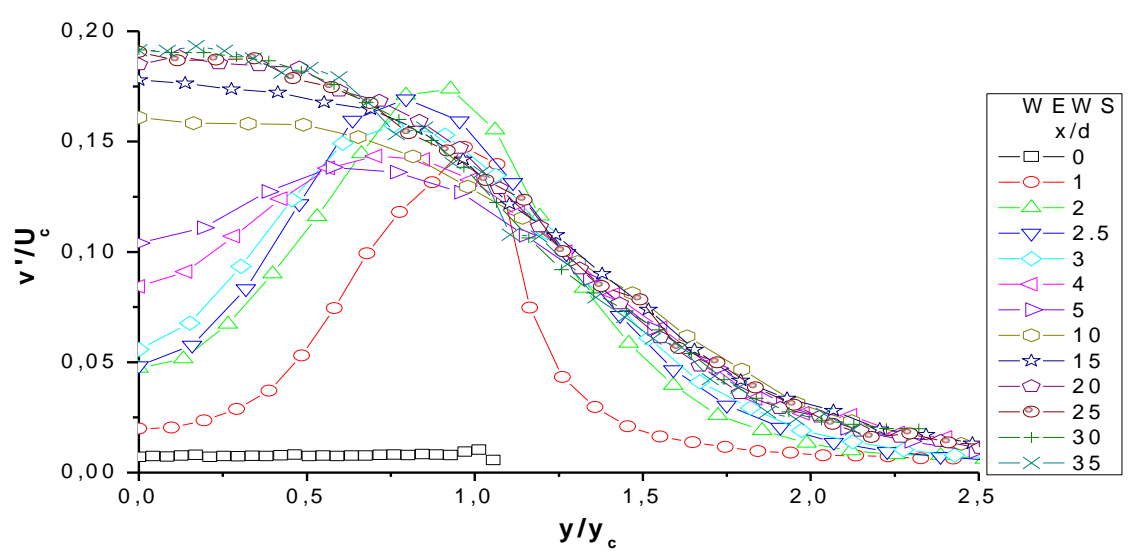

Figure 5.17: Self similarity of turbulent intensity distributions of the lateral velocity component on the central $x y$ plane for different test cases: a) NENS, b) WENS, c) NEWS and d) WEWS (cont'd).

\section{c) Transverse distributions of lateral turbulent velocities normalized to the exit velocity}

The transverse distributions of transverse turbulent velocity for all test cases are shown in Figures 5.18-a, b, $\mathrm{c}$ and d for downstream locations $x / D=1,4,15$ and 30 respectively. They are normalized to the exit streamwise velocity. The trends found here follow the ones of $\mathrm{u}^{\prime}$ when normalized to $\mathrm{U}_{0}$ (Figures 5.13). Again the distributions are classified into two group based on the presence or absence of sidewalls.

a)

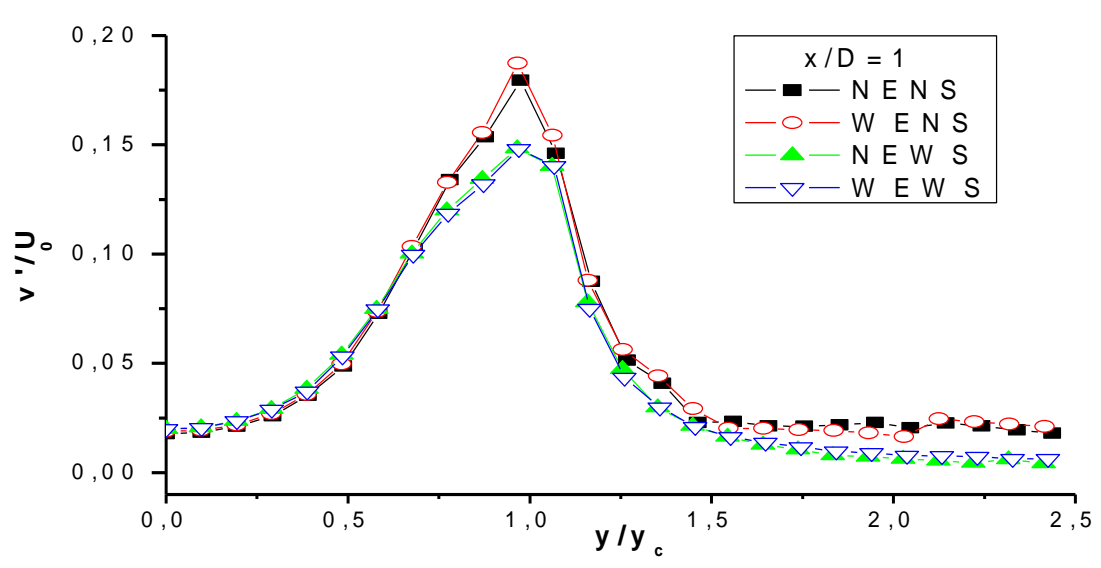

Figure 5.18: Turbulent intensity distributions of the lateral velocity component on the central $x y$ plane normalised to the exit velocity at different downstream locations. 
b)

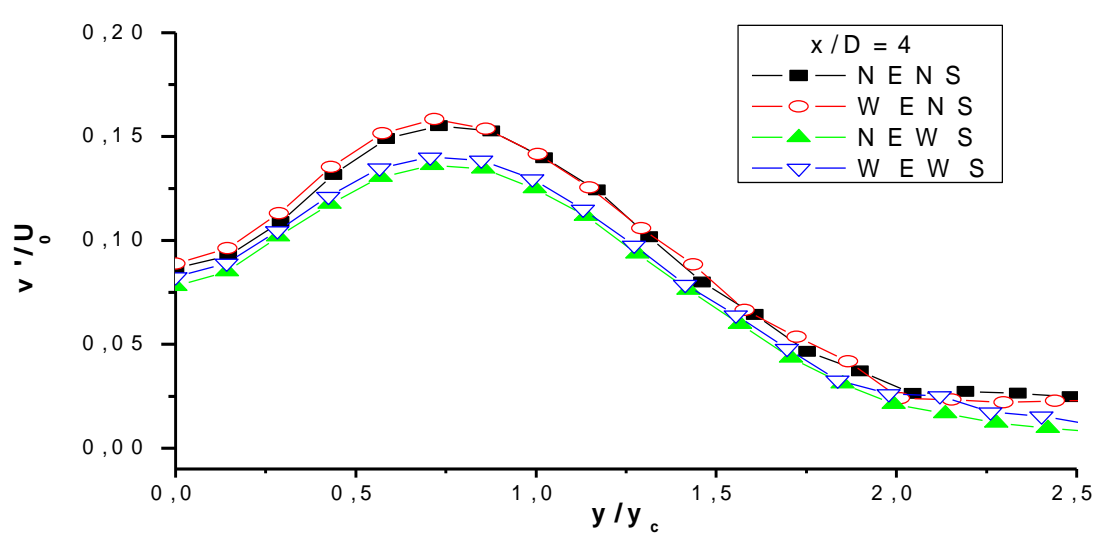

c)

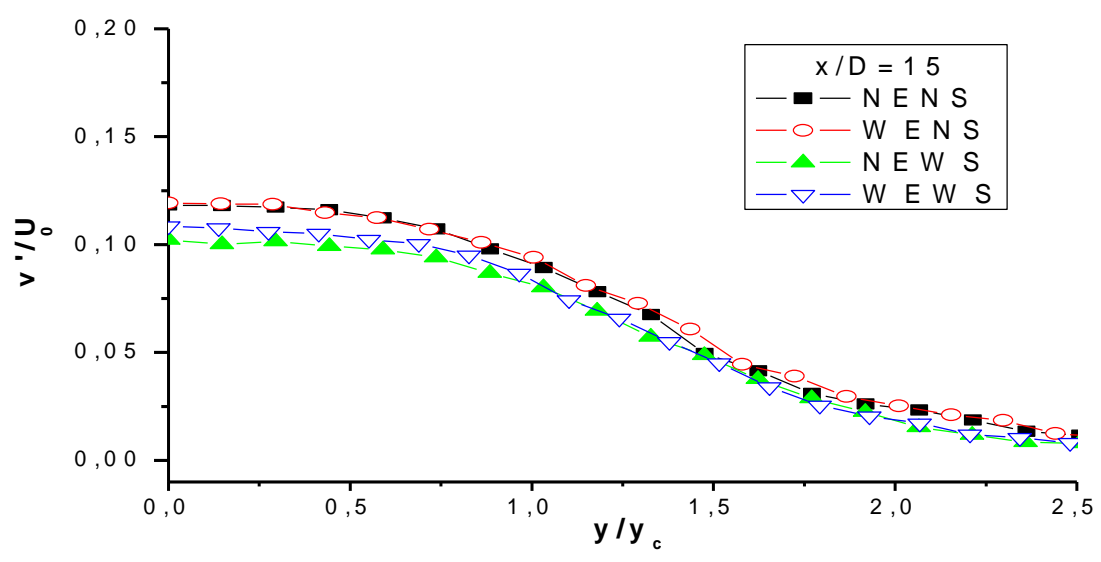

d)

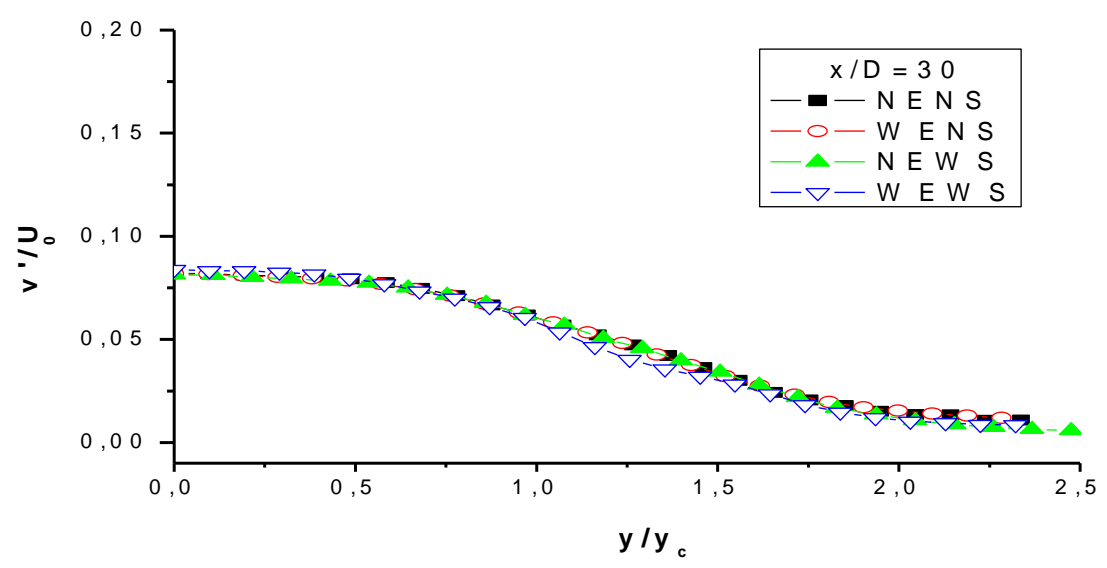

Figure 5.18: Turbulent intensity distributions of the lateral velocity component on the central $x y$ plane normalised to the exit velocity at different downstream locations (cont'd). 


\section{d) Evolutions of the centreline lateral turbulent velocity at various $\mathrm{Re}$}

To further study the development of turbulent transverse velocity for the four configurations, centreline distributions of $v^{\prime}$ at $R e=10,000,20,000$ and 30,000 up to $x / D=35$ are presented in Figures 5.19-a, $\mathrm{b}$ and c respectively. The trends are found to be different to the corresponding trends of longitudinal turbulent velocity (see Figure 5.14).

At $R e=10,000$ (Figure 5.19-a), $v^{\prime}$ in the four jets develops at nearly similar rate in the initial stages but further downstream beyond $x / D=10$ trends are different. In the absence of the sidewalls, the distributions of NENS and WENS are nearly similar at all Re tested indicating insignificant effect of the endplate on $v^{\prime}$ of jet without sidewalls. But in the presence of sidewalls and absence of endplate, the magnitudes of $v^{\prime}$ showed the lowest values whereas for WEWS, the values are similar to NENS and WENS except at the furthest locations. At $R e=20,000$, again, similar trends are observed at initial stages as at $R e=10,000$, but further downstream beyond $x / D \sim 10$, trends are different. A monotonic increase of $v^{\prime}$ is observed in NEWS and WEWS. This trend has been also observed in the transverse measurements (Figure $5.17 \mathrm{c}$ and d). At $R e=30,000$, the trends of the four configurations are different in comparison to the trends of $R e=10,000$ and 20,000. A peak value of $v^{\prime}$ is observed in all configurations. The magnitude of this peak is found to depend on the configuration. The highest peak is found for WEWS while the lowest is found for NEWS. The peaks of NENS and WENS are nearly similar indicating insignificant effect of the endplate on the jet without sidewalls.

a)

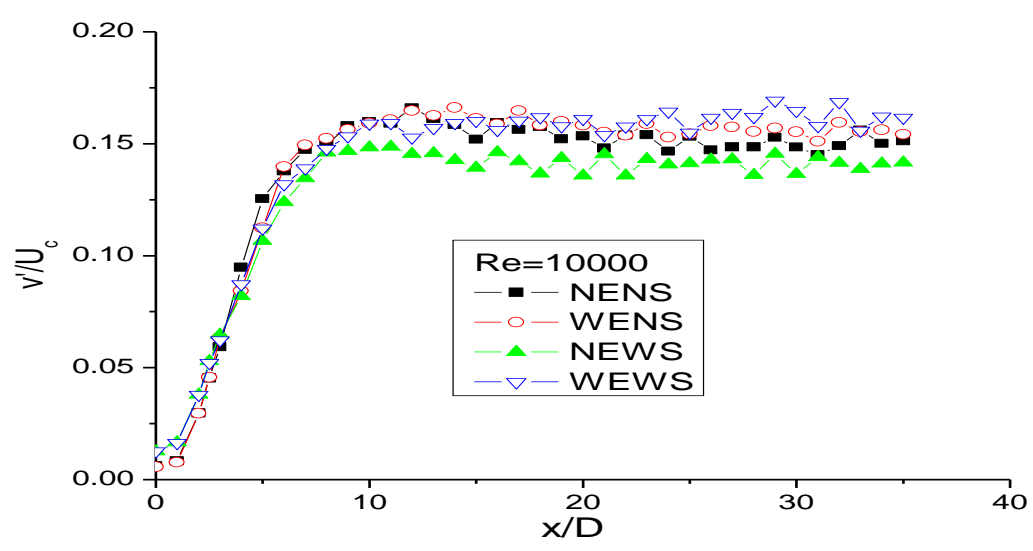

Figure 5.19: Centreline distributions of lateral turbulent velocity for all configurations at various Reynolds numbers: a) 10,000, b) 20,000 and c) 30,000. 
b)

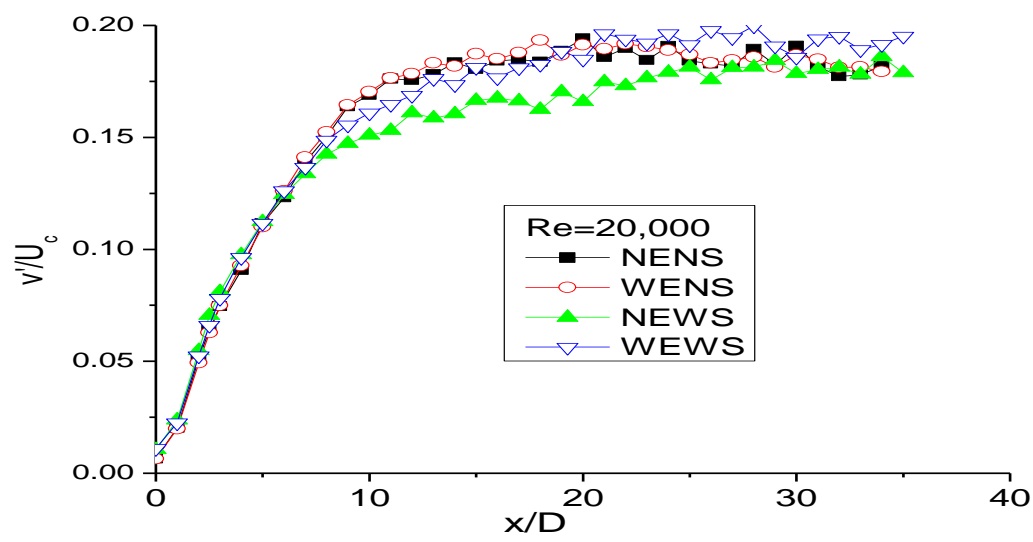

c)

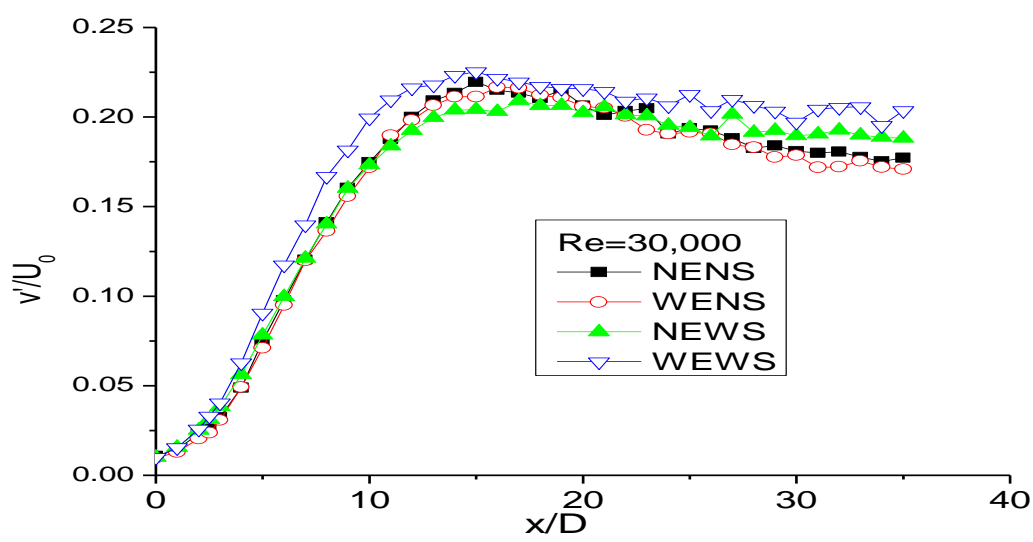

Figure 5.19: Centreline distributions of lateral turbulent velocity for all configurations at various Reynolds numbers: a) 10,000, b) 20,000 and c) 30,000 (con't).

The effect of Reynolds number on each configuration is demonstrated in Figures 5.20-a, b, c and d. Initially, for all configurations, a rapid increase of $v^{\prime}$ is observed, slower at the highest Reynolds number (i.e. $R e=30,000$ ). Further downstream, i.e. beyond $\sim 10$ nozzle widths, the magnitude of $v^{\prime}$ of the jets without sidewalls (NENS, WENS) increases with $R e$. And the magnitude of $v^{\prime}$ seems to attain its asymptotic value except at $R e=30,000$ where a monotonic decrease is observed. For configurations incorporating sidewalls, in the near field, a rapid increase is observed, slower at $R e=30,000$. Beyond $\sim x / D=10$, the magnitudes of $v^{\prime}$ at $R e=10,000$ indicate a saturation, but at $R e=20,000$ and 30,000, a monotonic increase and decrease are observed respectively. A peak value of $v^{\prime}$ at about 14 nozzle width, is observed only at $R e=30,000$ for all configurations. 
These measurements imply that, for all configurations, Reynolds number is found to have an effect on the turbulent velocity field.

a)

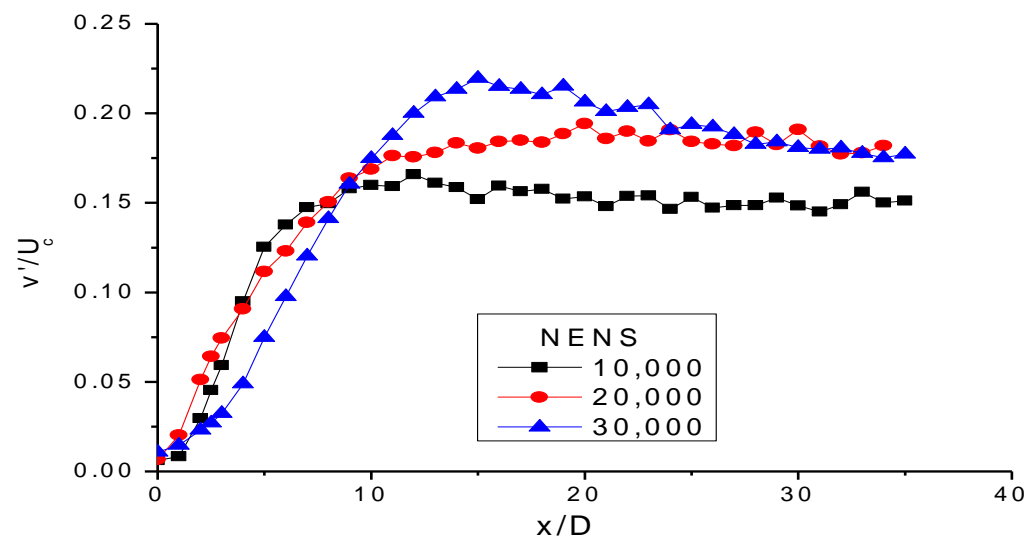

b)

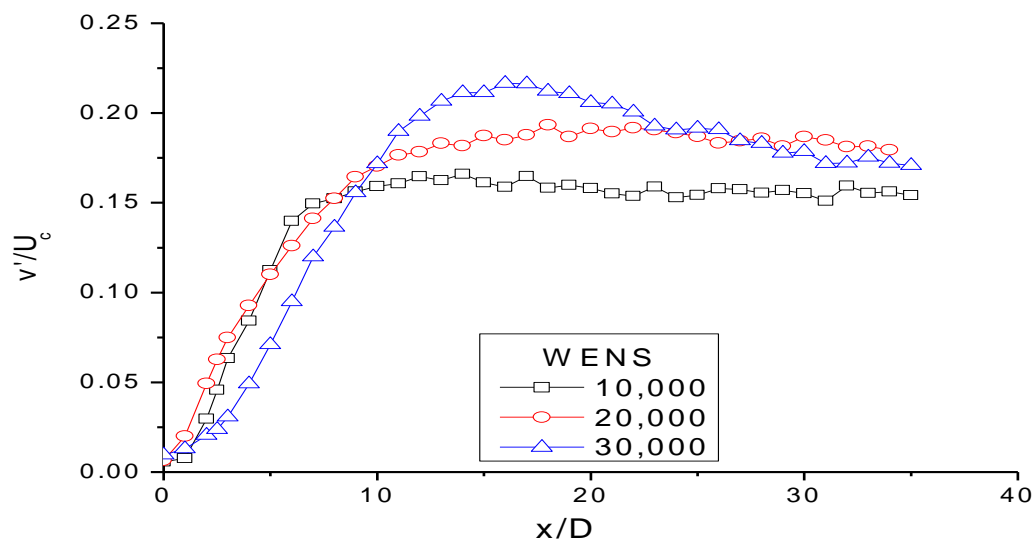

c)

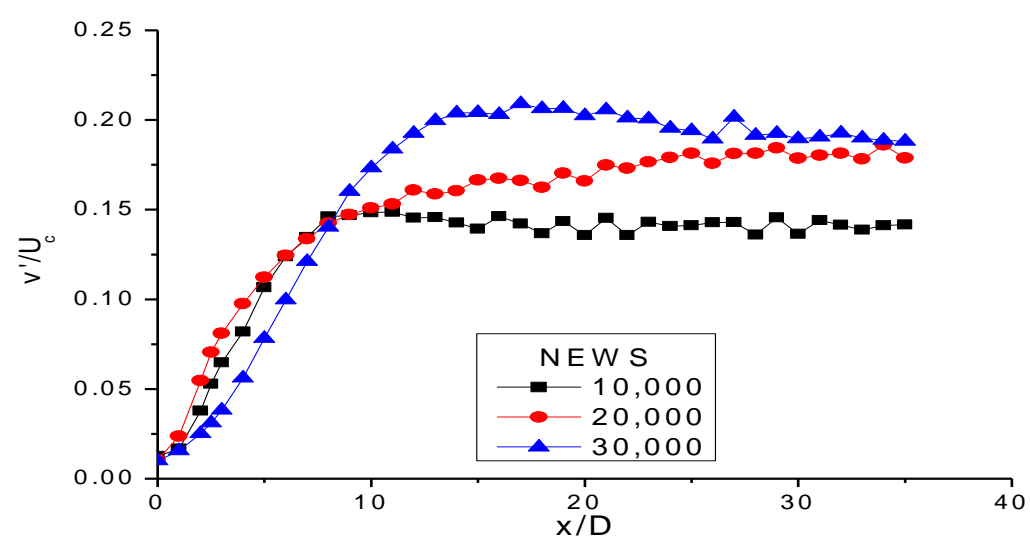

Figure 5.20: Reynolds number $R e$ dependence of the centerline lateral turbulent velocity for: a) NENS, b) WENS, c) NEWS and d) WEWS. 
d)

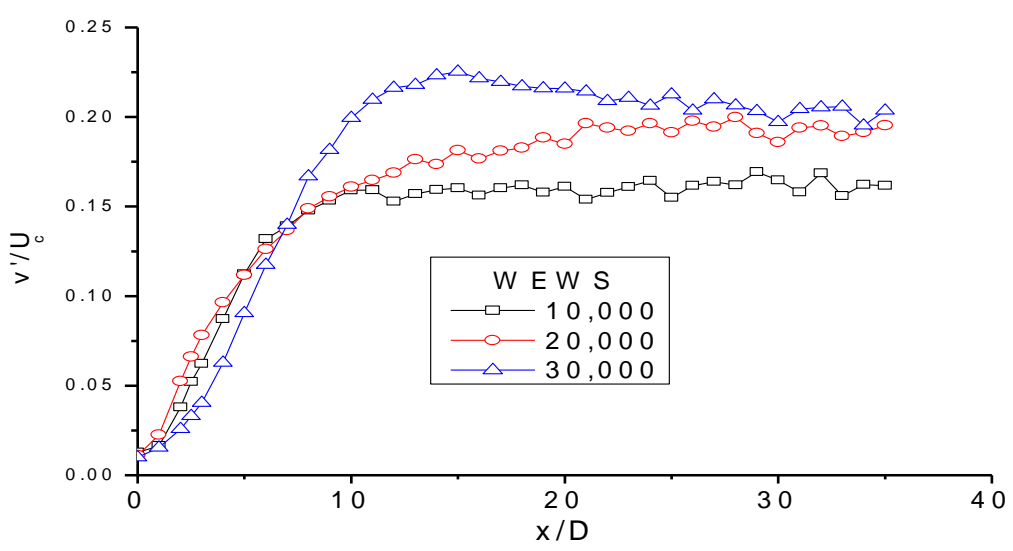

Figure 5.20: Reynolds number $R e$ dependence of the centerline lateral turbulent velocity for: a) NENS, b) WENS, c) NEWS and d) WEWS (con't).

\subsubsection{Reynolds shear stresses}

\section{a) Transverse distributions of shear stresses normalized to the centreline velocity}

The impact of the endplate to the profiles of the Reynolds shear stress, $\langle u v\rangle$, normalised by $U_{c}^{2}$, on the central $x y$ plane at $x / D=0,1,2,2.5,3,4,5,10,15,20,25$, 30 and 35 for all test cases, shown in Figures 5.21-a, b, c, d, e, f, g, h, j, k, 1, m and n respectively, is similar to that on the corresponding fluctuating velocities' moment. Figure 5.22 depicts the self-preserving behaviour of the four jets which is also similar to fluctuating velocities.

a)

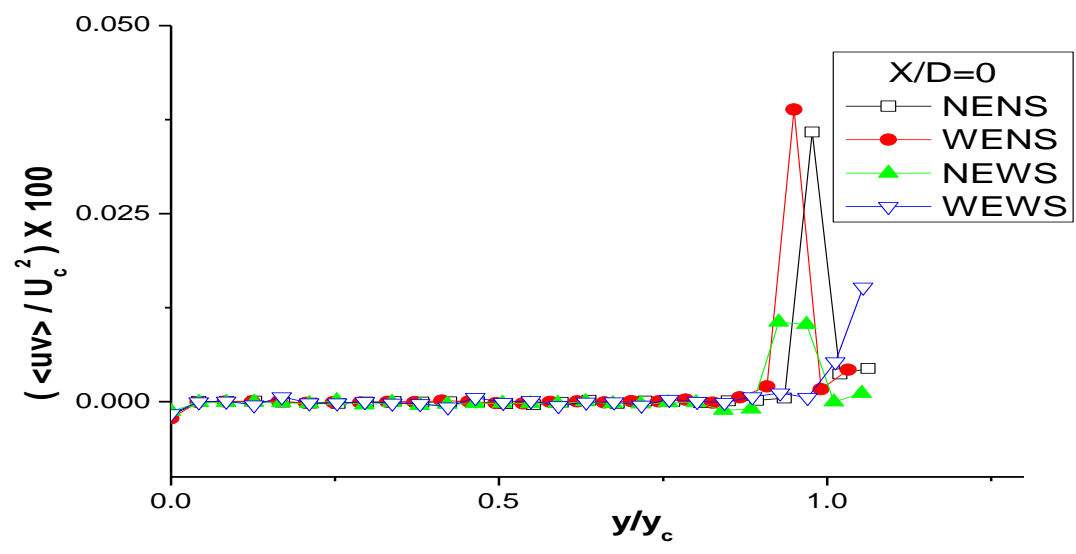

Figure 5.21: Reynolds shear stress profiles on the central $x y$ plane normalized to the centreline velocity at different downstream locations. 
b)

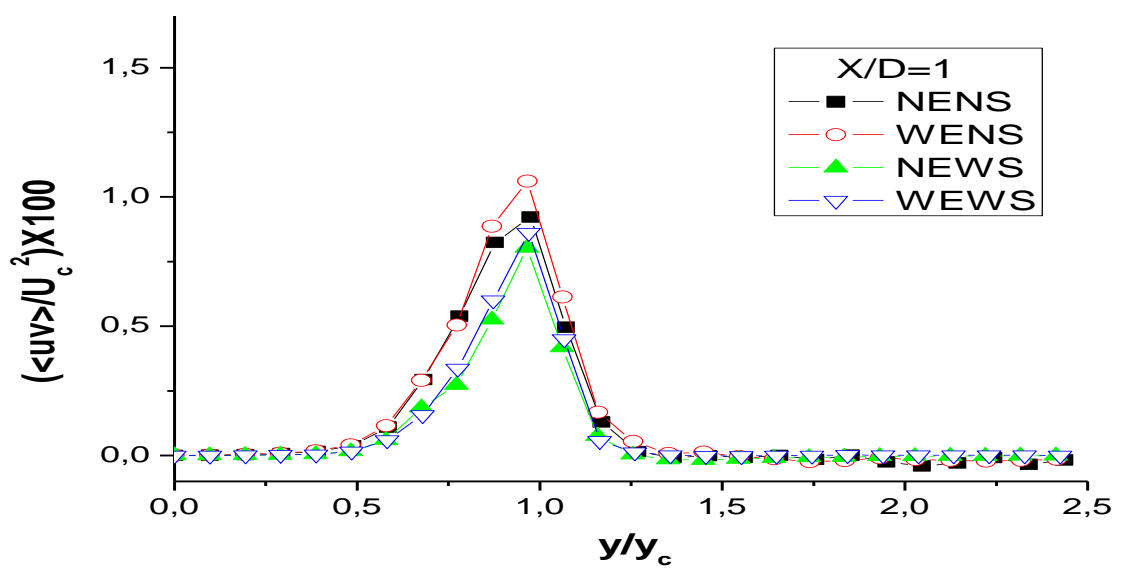

c)

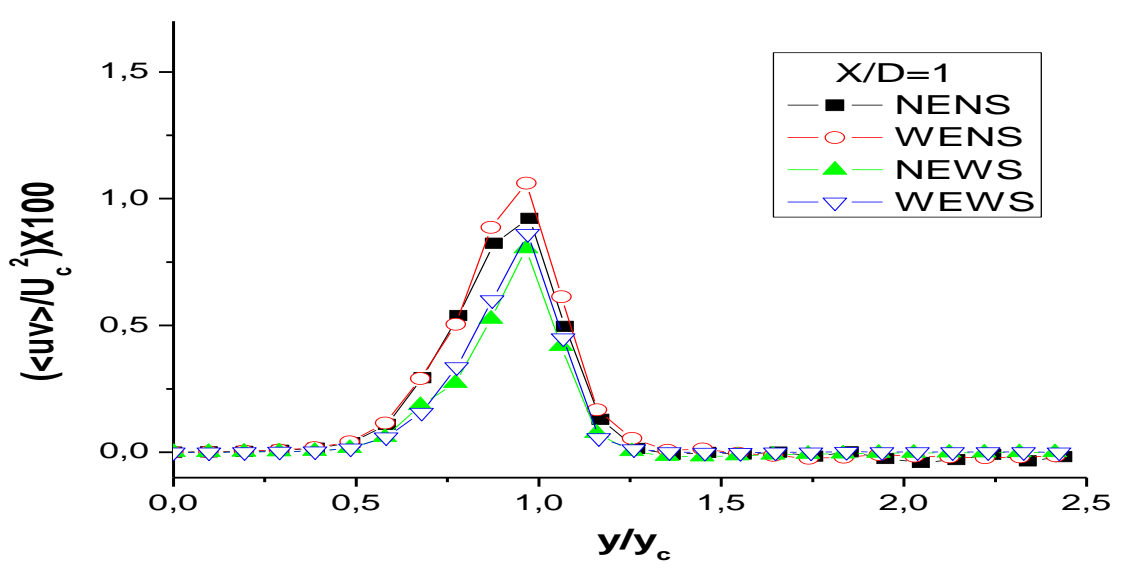

d)

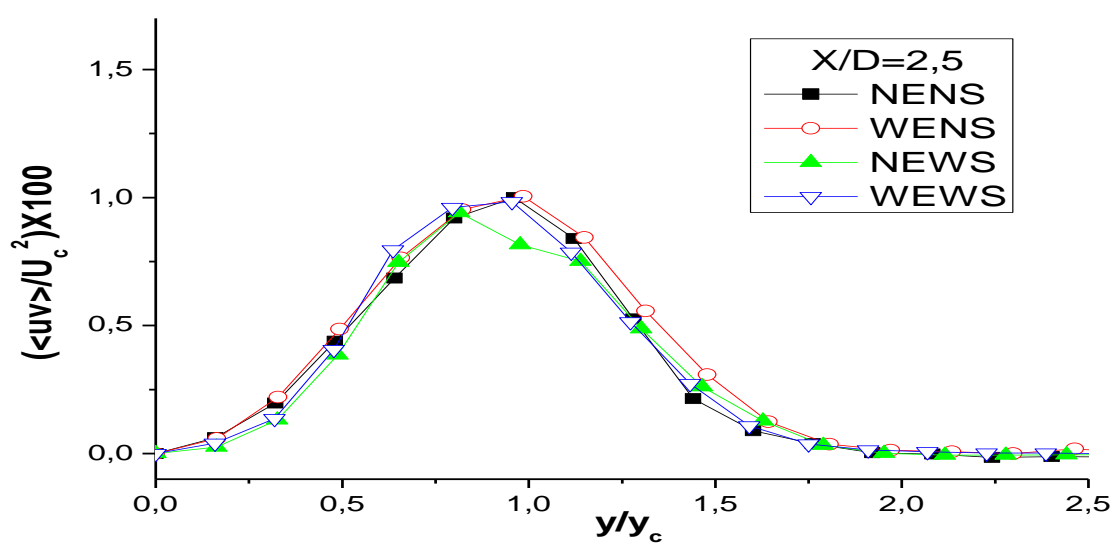

Figure 5.21: Reynolds shear stress profiles on the central $x y$ plane normalized to the centreline velocity at different downstream locations (cont'd). 
e)

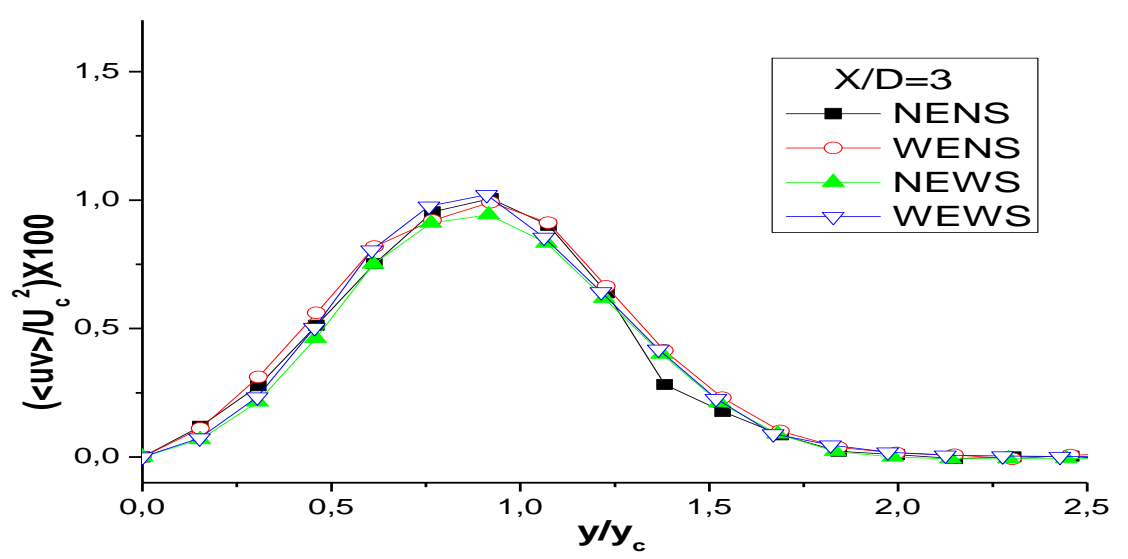

f)

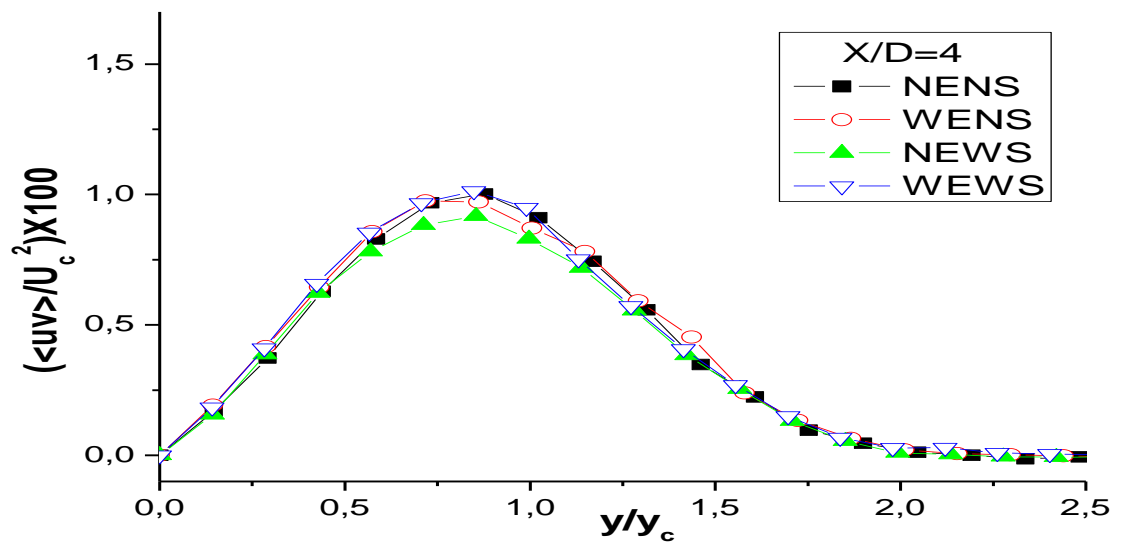

g)

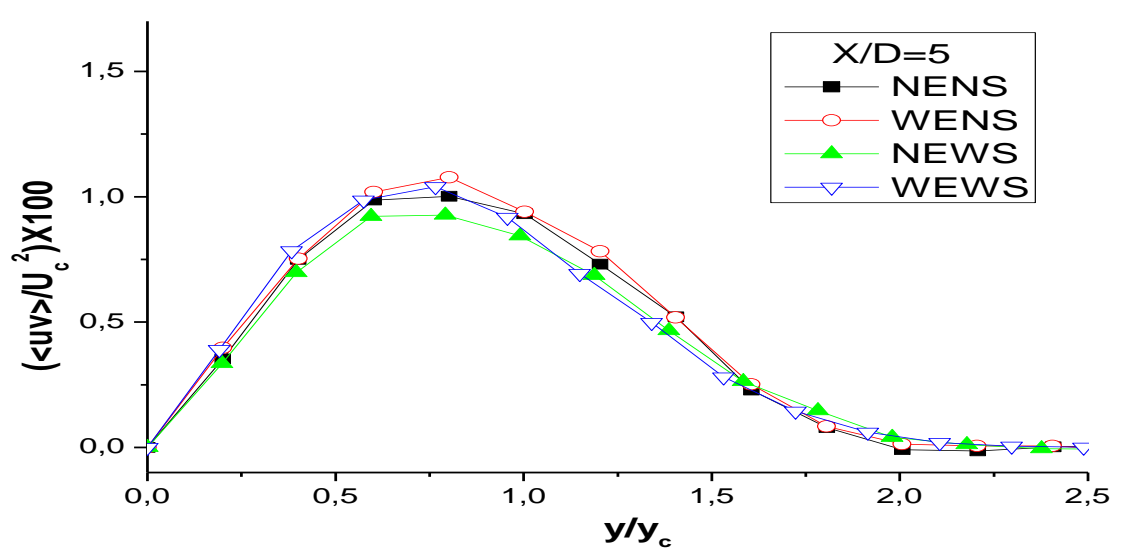

Figure 5.21: Reynolds shear stress profiles on the central $x y$ plane normalized to the centreline velocity at different downstream locations (cont'd). 
h)

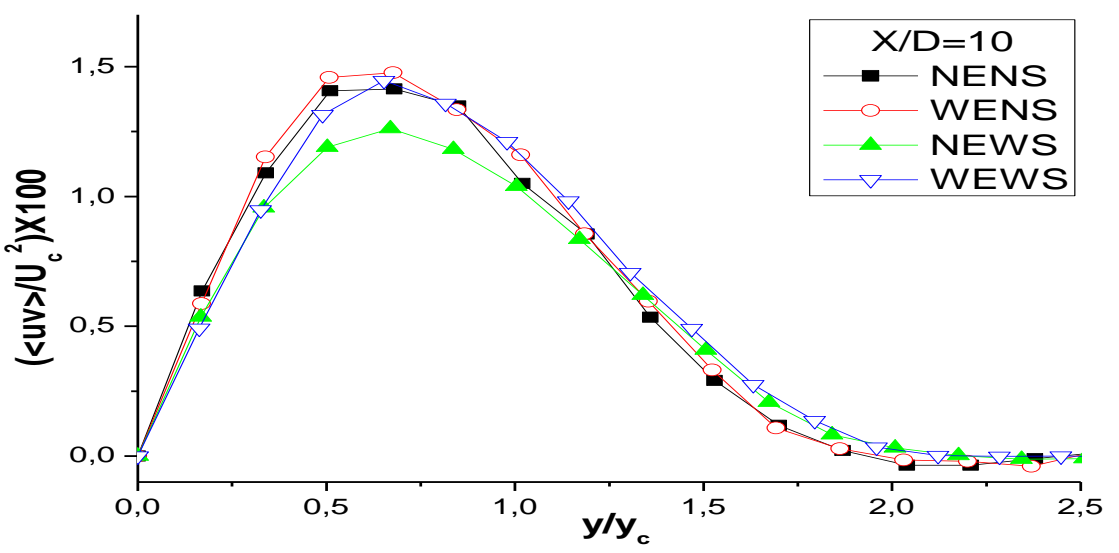

j)

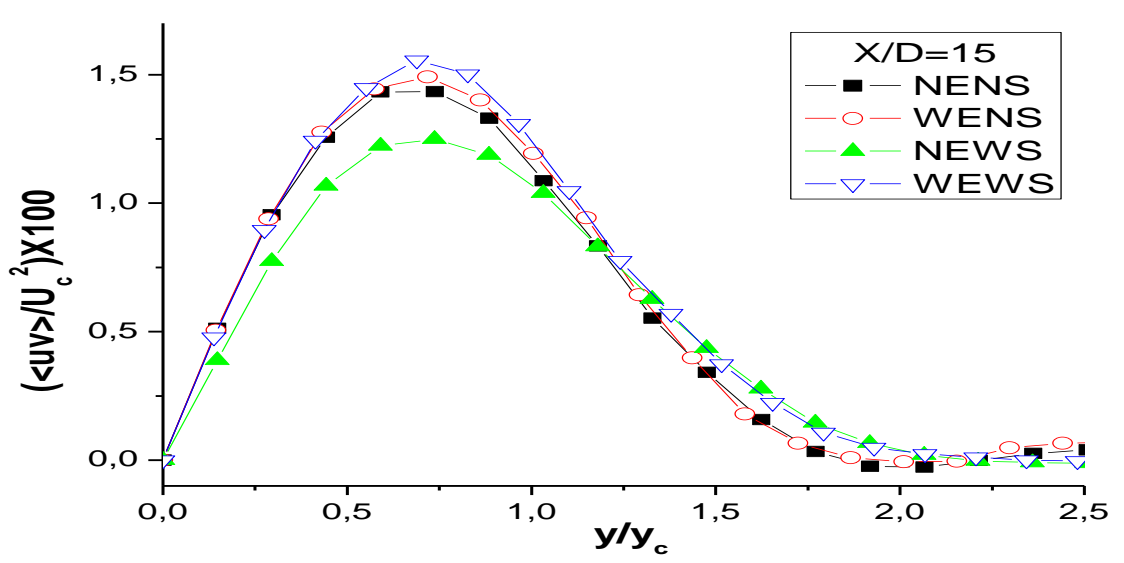

$\mathrm{k})$

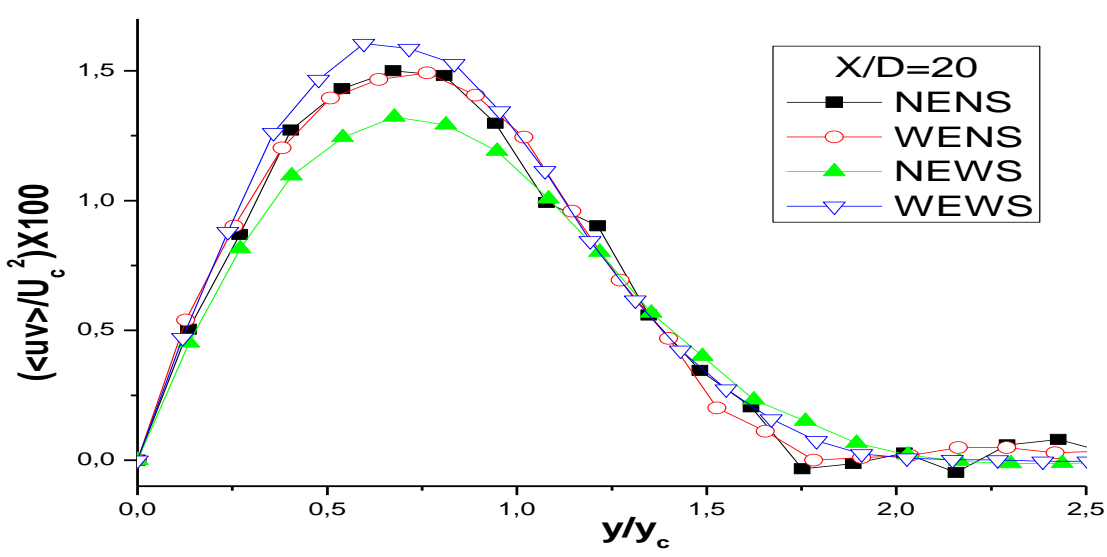

Figure 5.21: Reynolds shear stress profiles on the central $x y$ plane normalized to the centreline velocity at different downstream locations (cont'd). 
1)

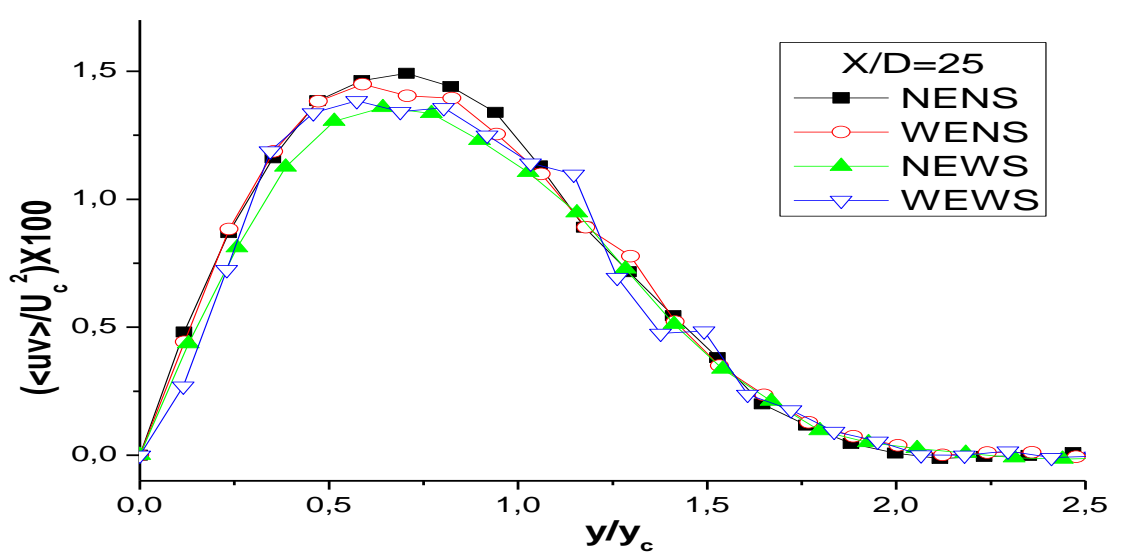

m)

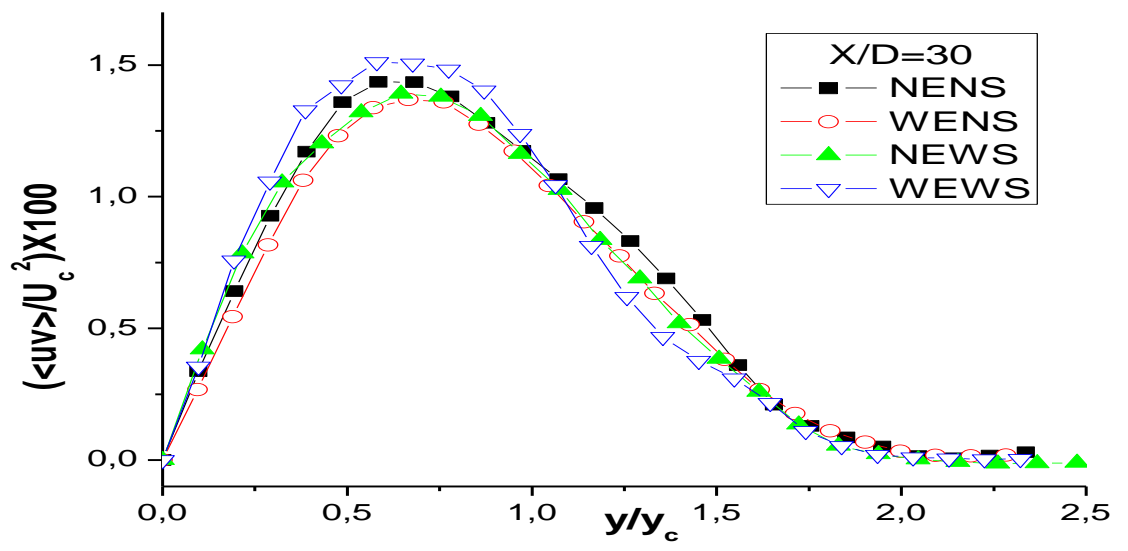

n)

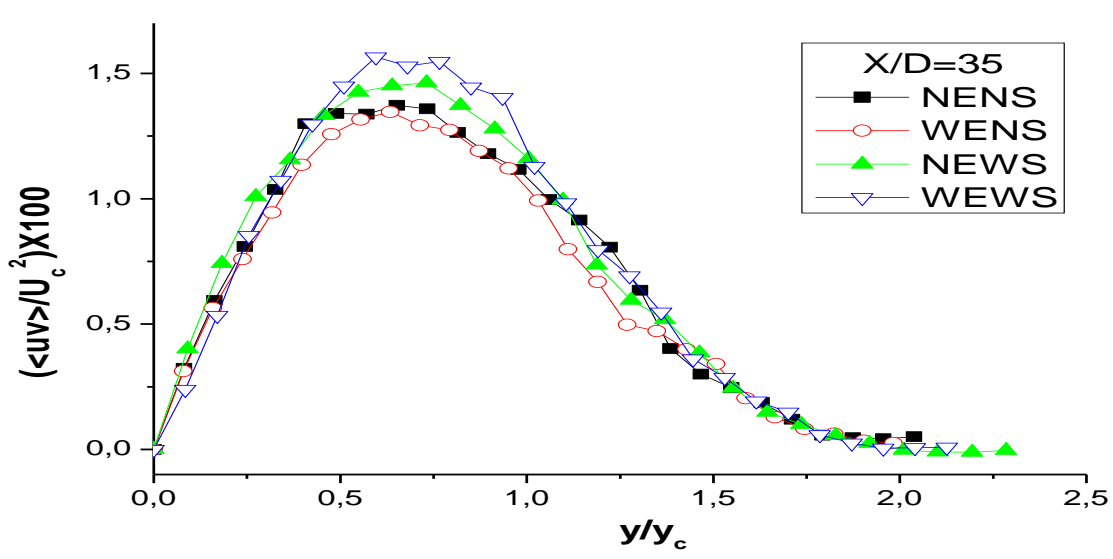

Figure 5.21: Reynolds shear stress profiles normalized to $U_{c}$ at different downstream locations (cont'd). 
a)

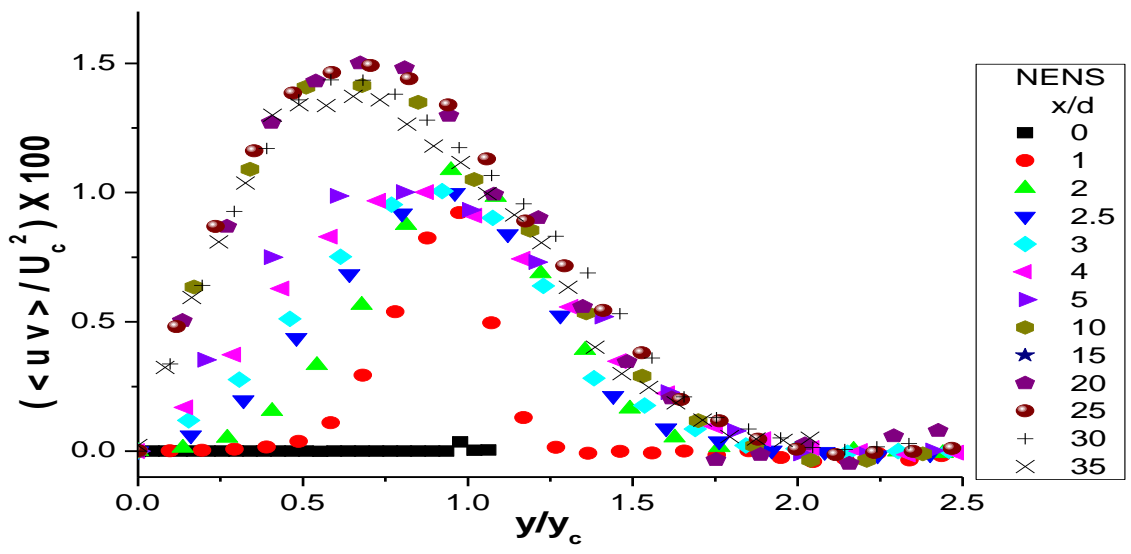

b)

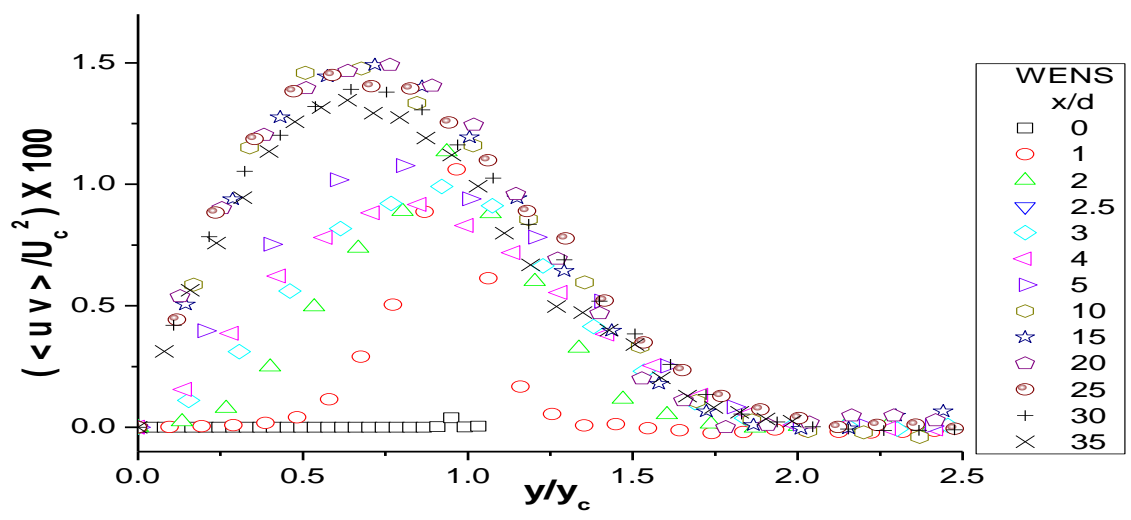

c)

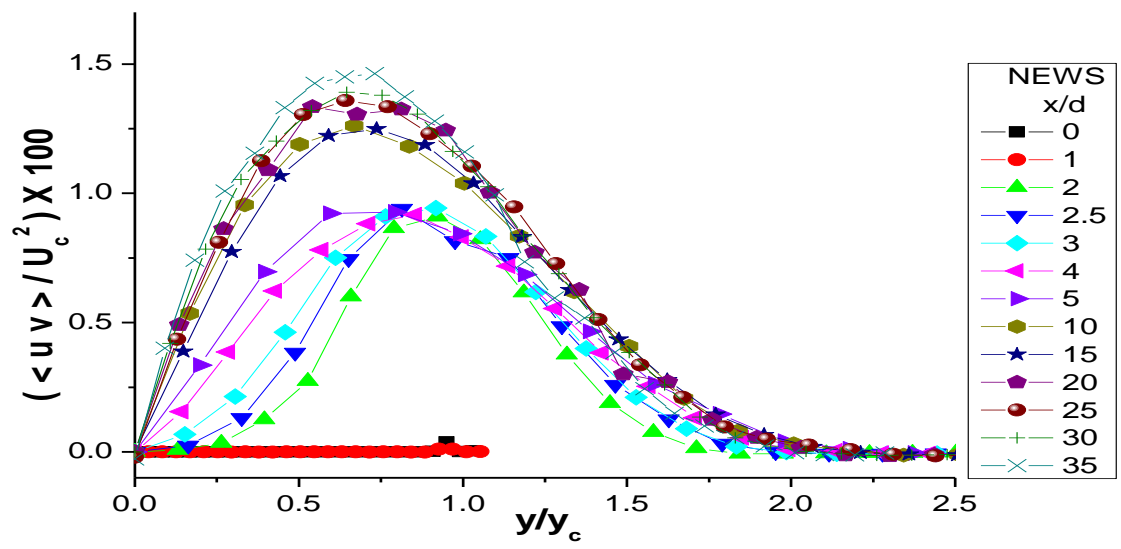

Figure 5.22: self similarity profiles of Reynolds shear stress on the central $x y$ plane for all test cases: a) NENS, b) WENS, c) NEWS and d) WEWS. 
d)

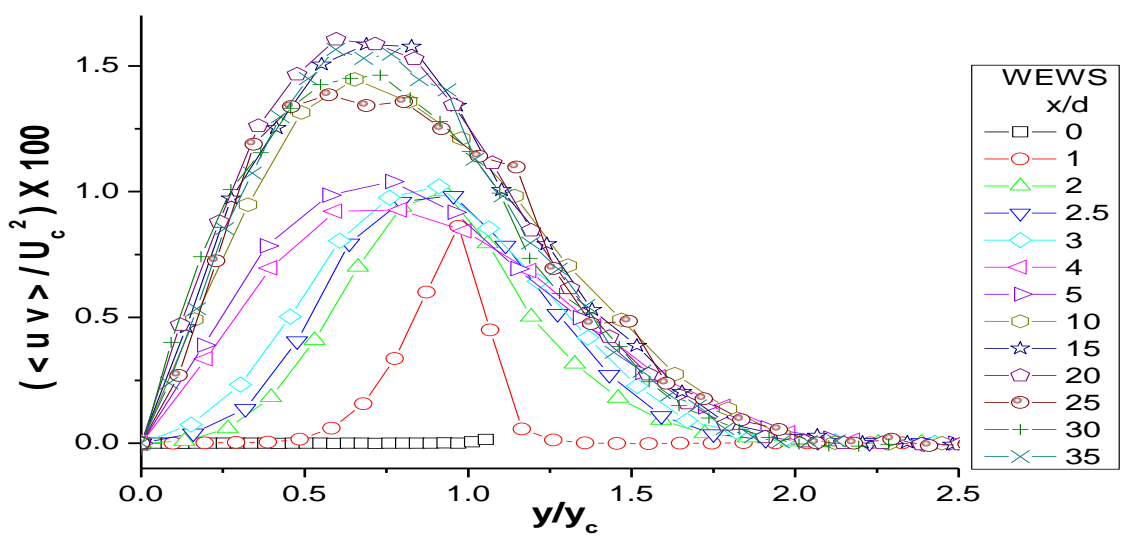

Figure 5.22: self similarity profiles of Reynolds shear stress on the central $x y$ plane for all test cases: a) NENS, b) WENS, c) NEWS and d) WEWS (cont'd).

\section{b) Transverse distributions of Reynolds shear stresses normalized to $u^{\prime} v^{\prime}$}

It is interesting to observe that when Reynolds shear stress is normalised by $u^{\prime} v^{\prime}$, depicting the correlation of the fluctuations, the distributions can be classified in two groups depending on the presence or absence of the sidewalls as shown in Figures 5.23-a, b, c and d for downstream locations $x / D=1,4,15$ and 30 respectively. In these graphs only minor differences at the furthest from the centreline points, outside the edges of the jet, could be associated to the presence or absence of the endplate, which, as already mentioned, may be partly attributed to the increased uncertainty of hotwire measurements at these locations.

a)

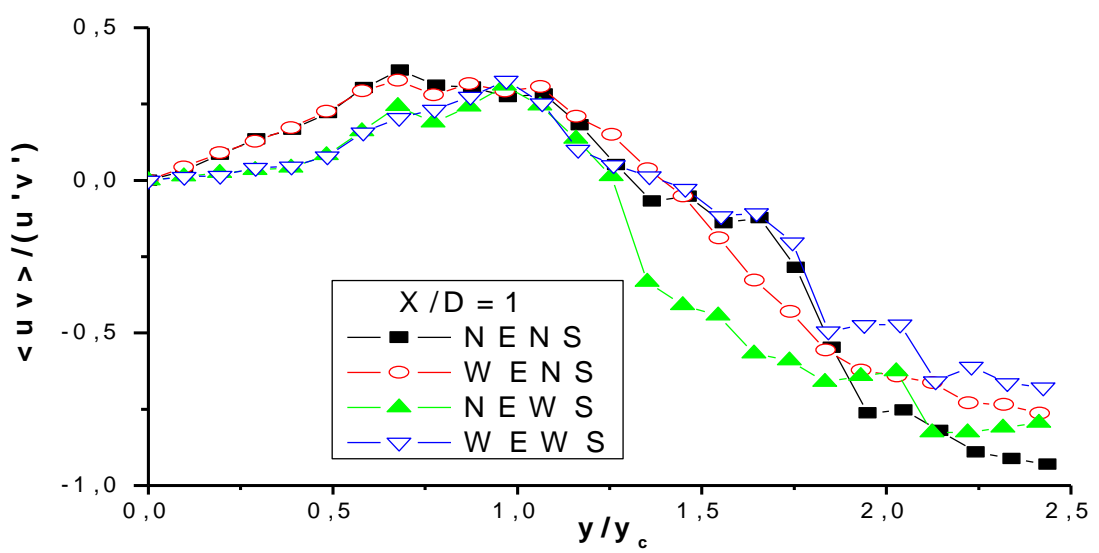

Figure 5.23: Reynolds shear stress profiles on the central $x y$ plane normalized to $\left(u^{\prime} v^{\prime}\right)$ at different downstream locations. 
b)

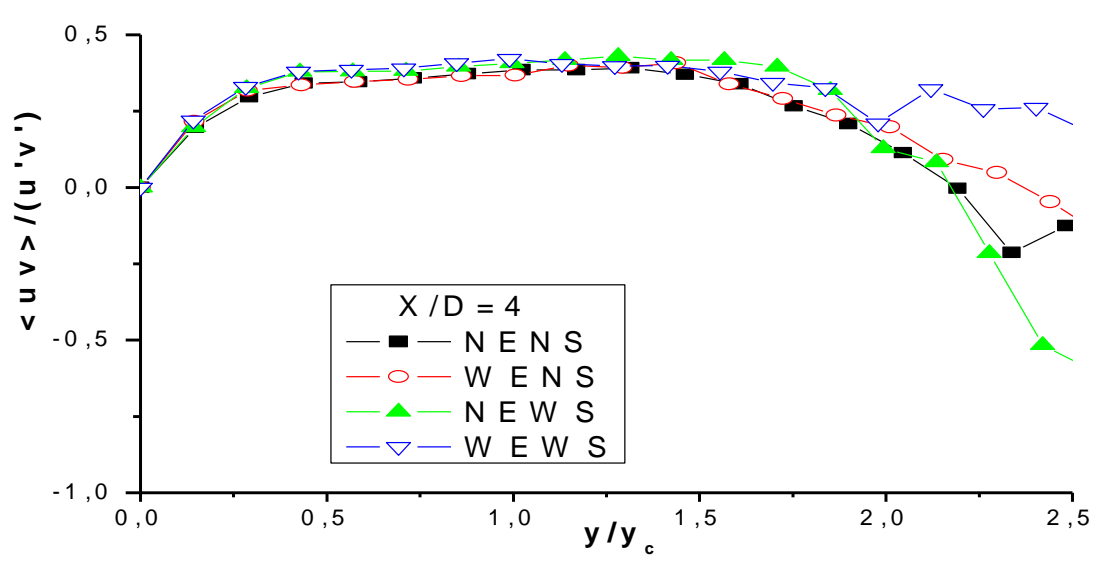

c)

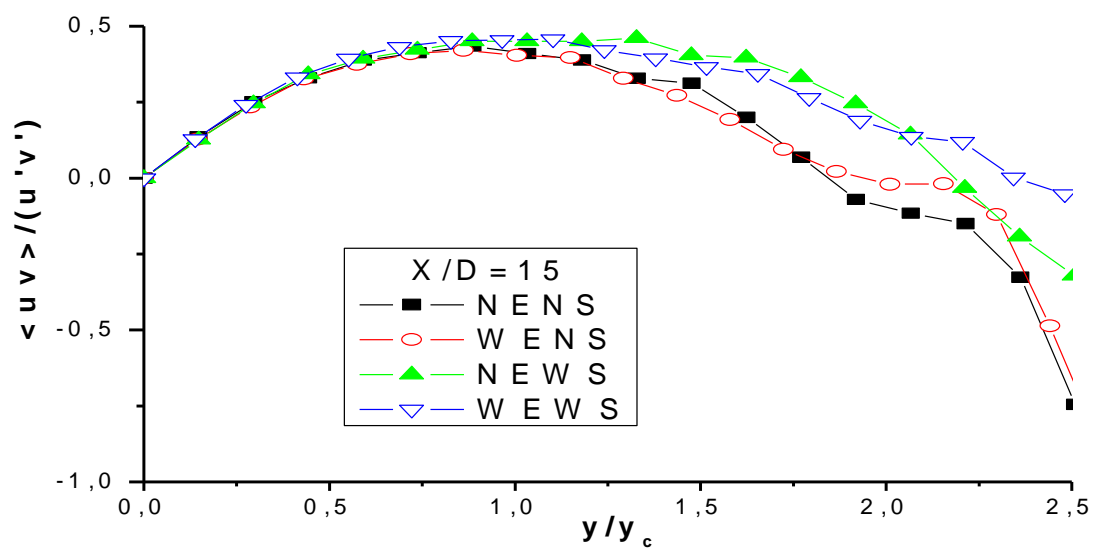

d)

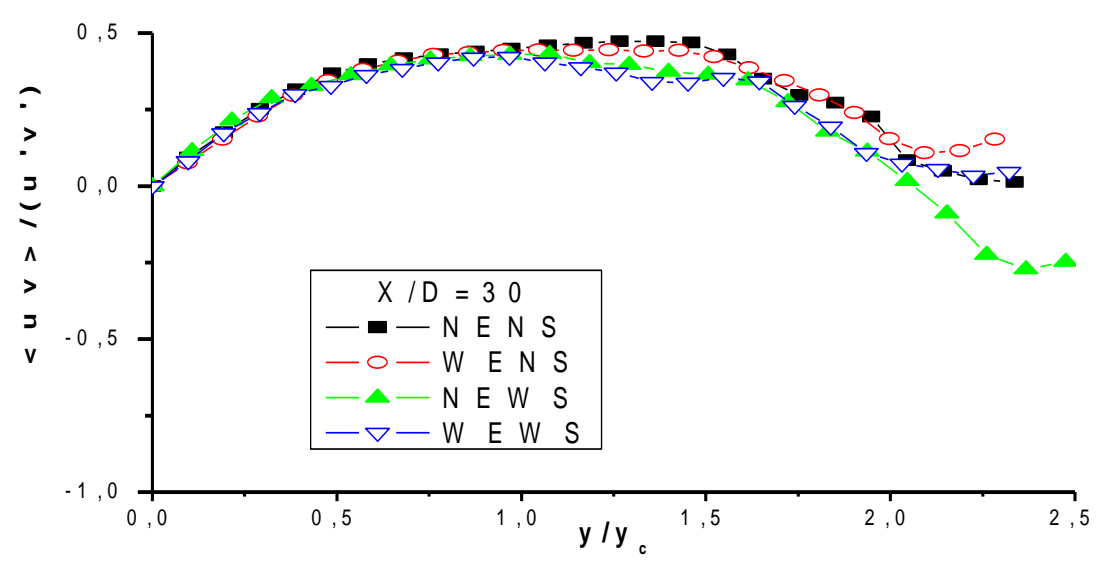

Figure 5.23: Reynolds shear stress profiles on the central $x y$ plane normalized to $\left(\mathrm{u}^{\prime} \mathrm{v}^{\prime}\right)$ at different downstream locations (cont'd). 


\subsubsection{Triple velocity products}

a) Transverse distributions of triple velocity product $\left\langle u^{3}\right\rangle$

The streamwise velocity triple product $\left\langle u^{3}\right\rangle$ normalized by $U_{c}^{3}$ on the central $x y$ plane for the four configurations are shown in Figures 5.24-a, b, c, d, e, f, g, h, j, k, 1, m and $\mathrm{n}$ for downstream locations of $x / D=0,1,2,2.5,3,4,5,10,15,20,25,30$, and 35 respectively.

Close to zero values are observed at positions neighbouring to those where $u^{\prime}$ attains maximum values. From these points towards the centre of the jet, values decrease reaching a negative minimum, close to the inward limit of the shear layer. In the early stages of jet development $\left\langle u^{3}\right\rangle$ is increasing closer to the centreline, attaining zero values in the potential core. Further downstream at $5 \mathrm{D}$ and $10 \mathrm{D}$ the distributions reach the centreline before attaining zero value, whereas at subsequent locations (25 $D$ and $35 D$ ) the negative minimum is observed on the jet axis. From the location of maximum $u^{\prime}$, the $\left\langle u^{3}\right\rangle$ values increase towards the edge of the jet reaching a positive maximum outside $y / y_{c}=1$, and decreasing to zero at larger distances from the centreline. Maximum values in this area, generally increase with $x / D$. The jets without sidewalls (NENS and WENS) present higher positive maxima until the downstream location of $10 \mathrm{D}$. At this location the jet with endplate and with sidewalls (WEWS) starts to show maxima in stations. Although the distributions of $\left\langle u^{3}\right\rangle$ for the jets without sidewalls (NENS and WENS) seem to have reached saturation, the other two jets with sidewalls (NEWS, WEWS) are still increasing as evidenced in Figure 5.24. Figures 5.25-a, b, c and d demonstrate the self preservation behaviour of $\left\langle u^{3}\right\rangle$ for configurations NENS, WENS, NEWS and WEWS respectively. 
a)

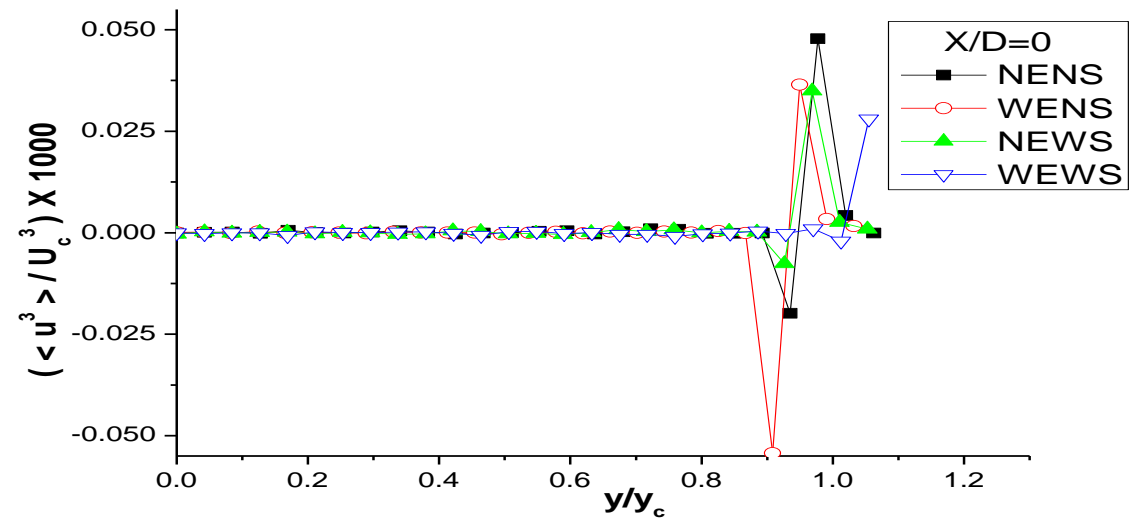

b)

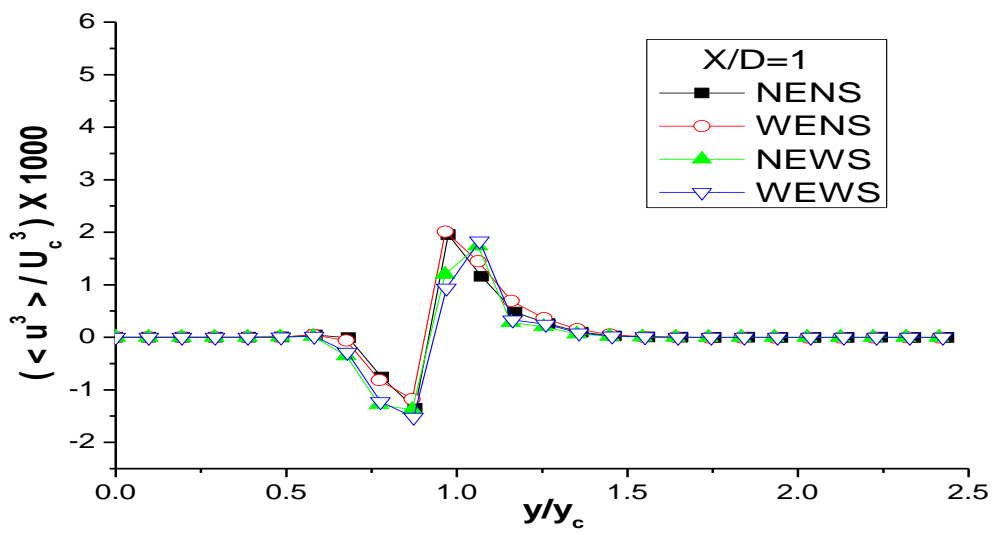

c)

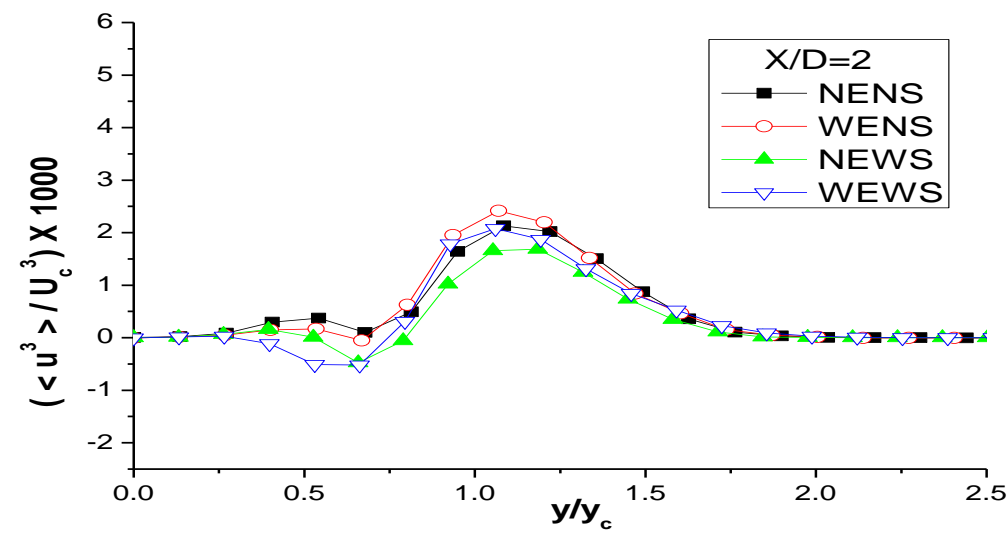

Figure 5.24: Profiles of triple velocity product of $\left\langle u^{3}\right\rangle$ on the central $x y$ plane at different downstream locations. 
d)

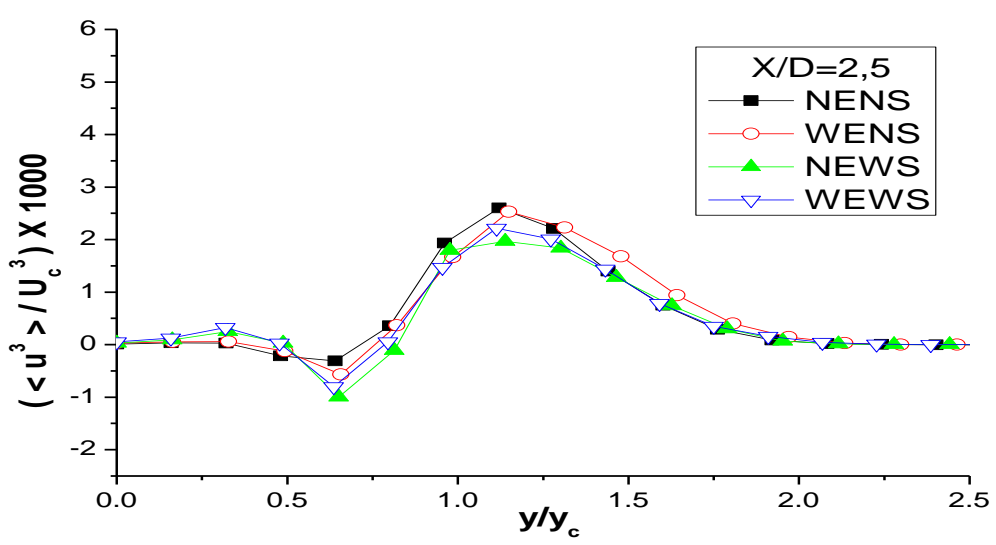

e)

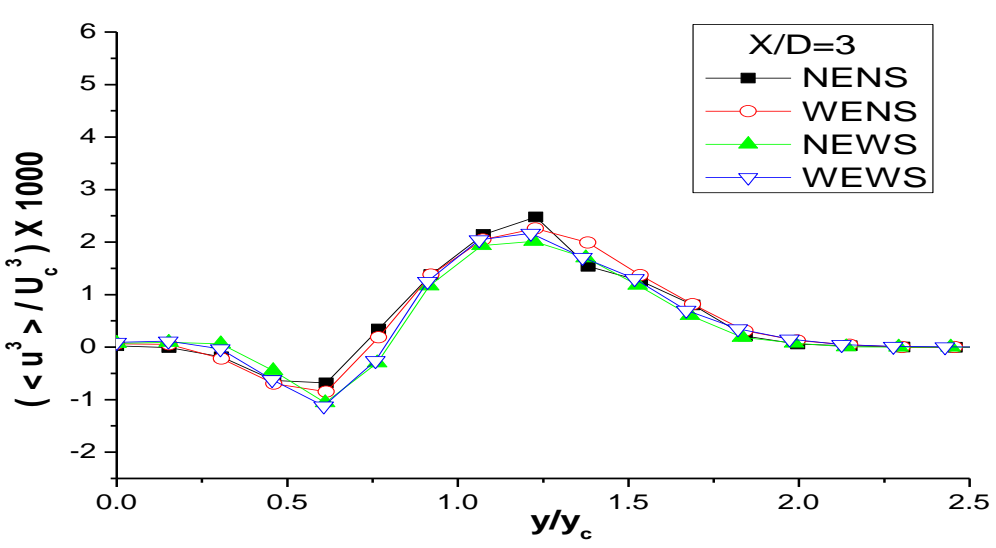

f)

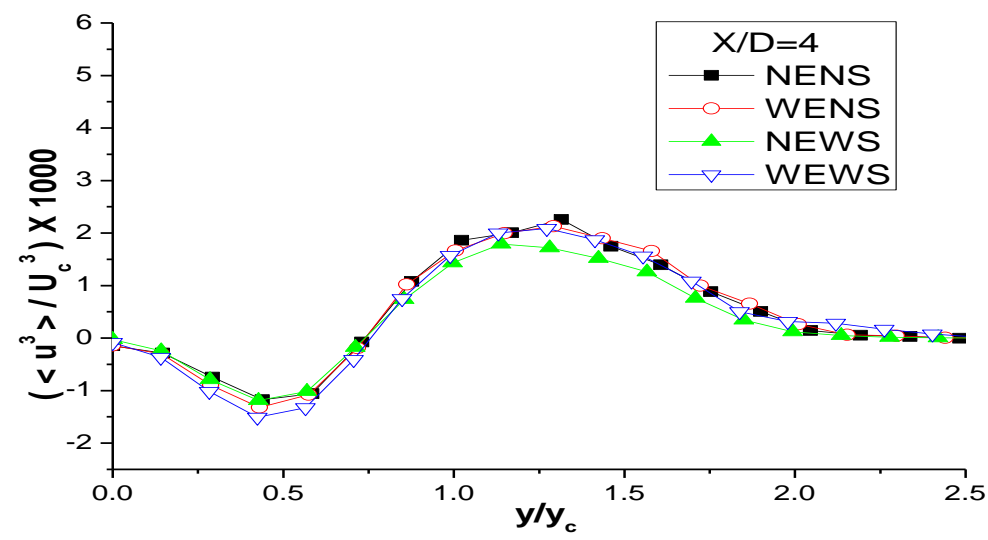

Figure 5.24: Profiles of triple velocity product of $\left\langle u^{3}\right\rangle$ on the central $x y$ plane at different downstream locations (cont'd). 
g)

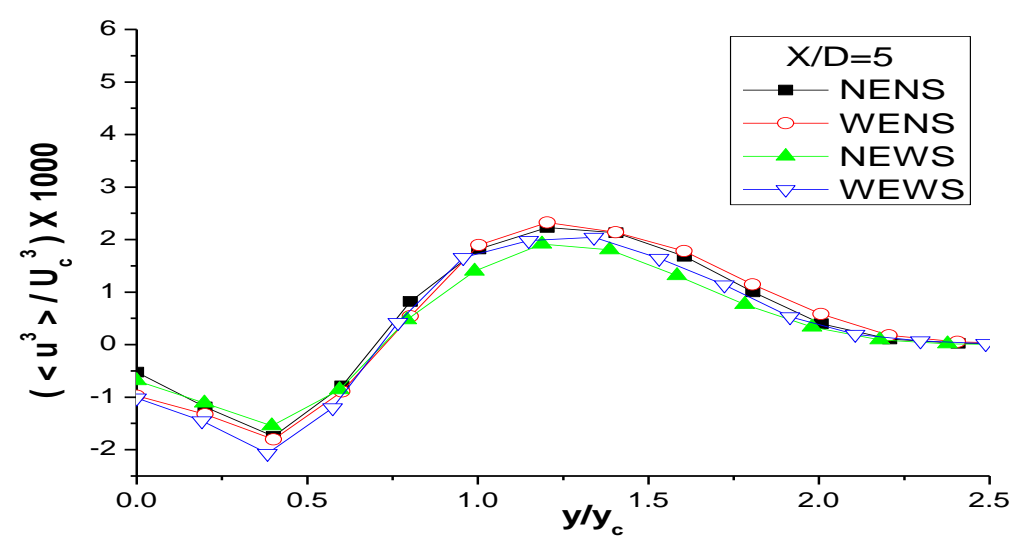

h)

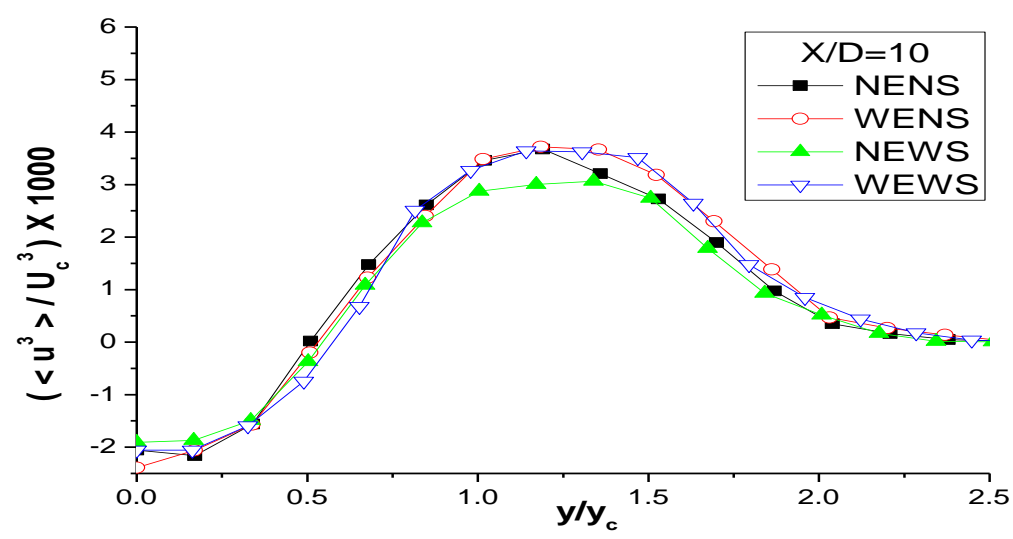

j)

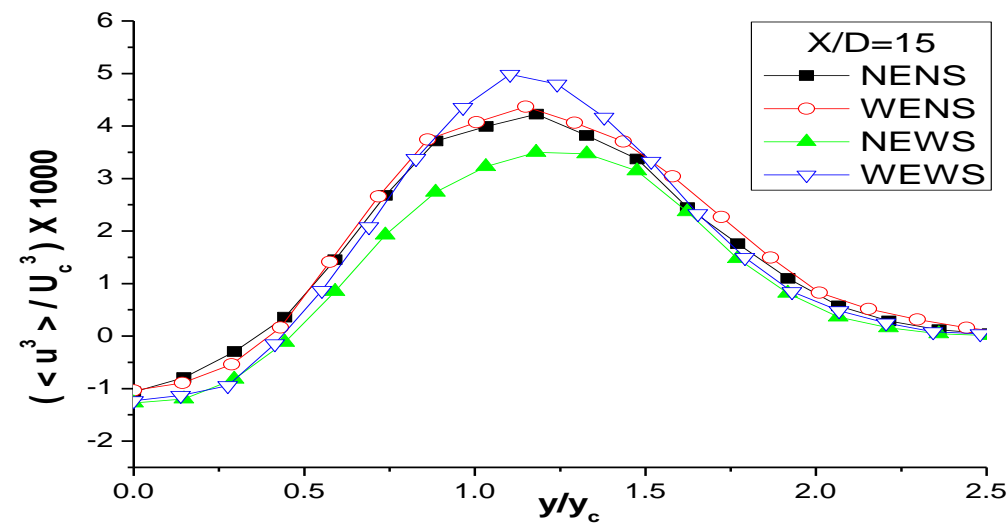

Figure 5.24: Profiles of triple velocity product of $\left\langle u^{3}\right\rangle$ on the central $x y$ plane at different downstream locations (cont'd). 
k)

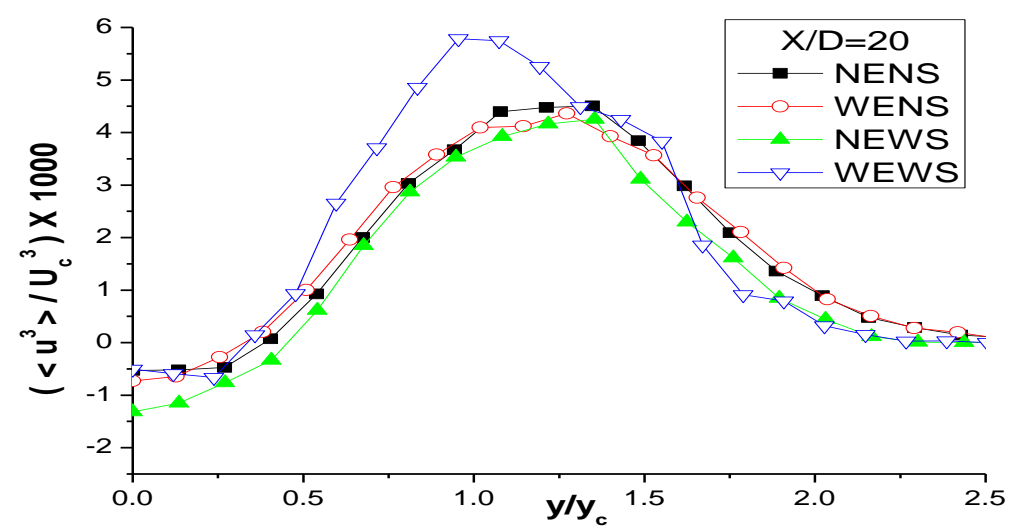

1)

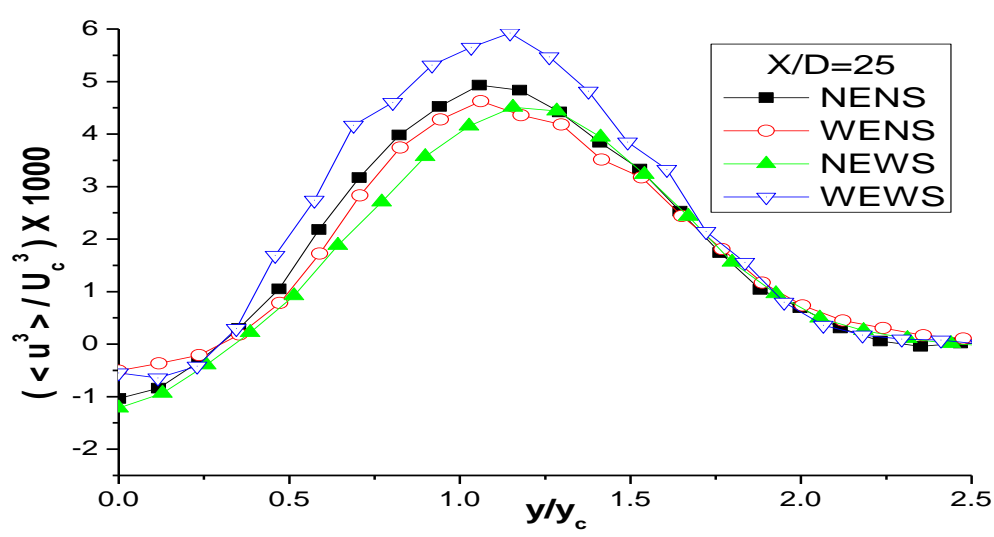

m)

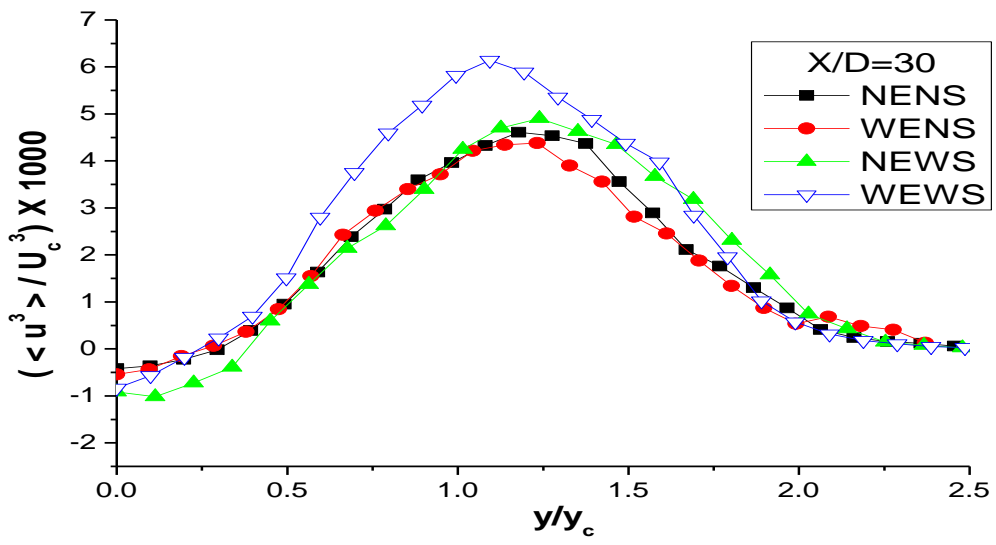

Figure 5.24: Profiles of triple velocity product of $\left\langle u^{3}\right\rangle$ on the central $x y$ plane at different downstream locations (cont'd). 
n)

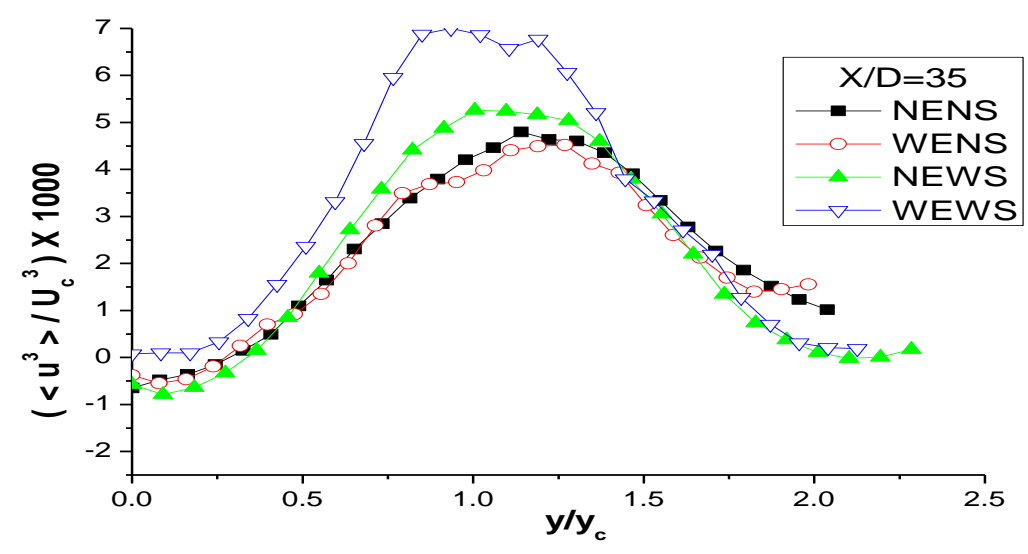

Figure 5.24: Profiles of triple velocity product of $\left\langle u^{3}\right\rangle$ on the central $x y$ plane at different downstream locations (cont'd).

a)

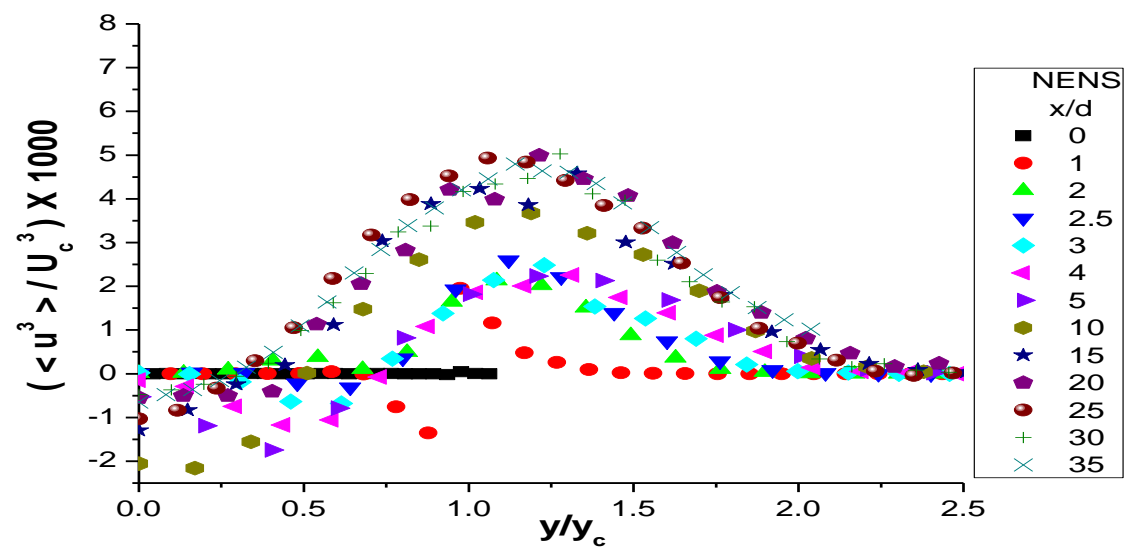

b)

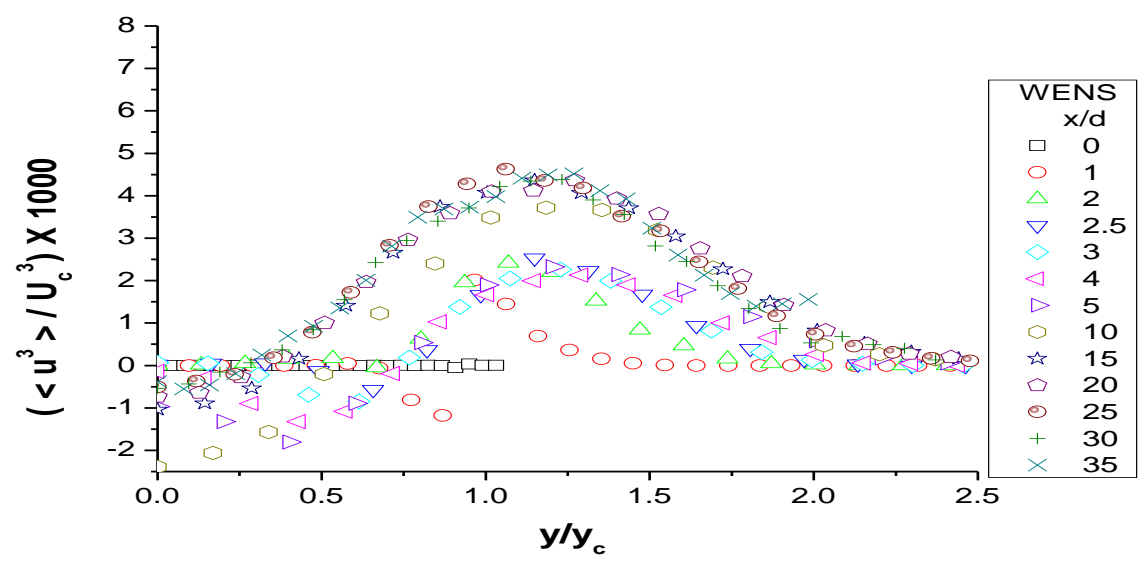

Figure 5.25: Self similarity profiles of triple velocity product $\left\langle u^{3}\right\rangle$ on the central $x y$ plane for all test cases: a) NENS, b) WENS, c) NEWS and d) WEWS. 
c)

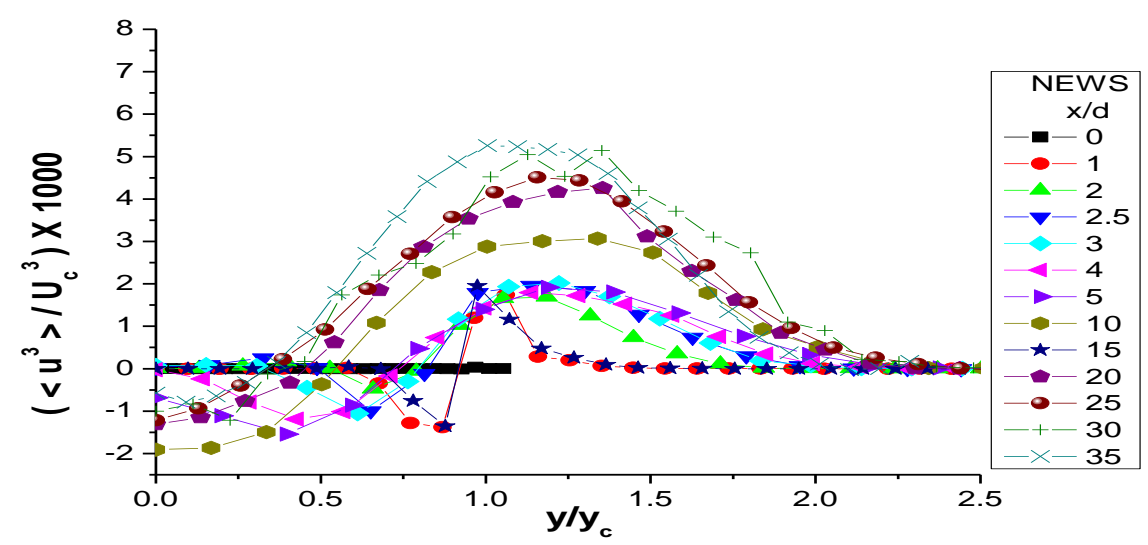

d)

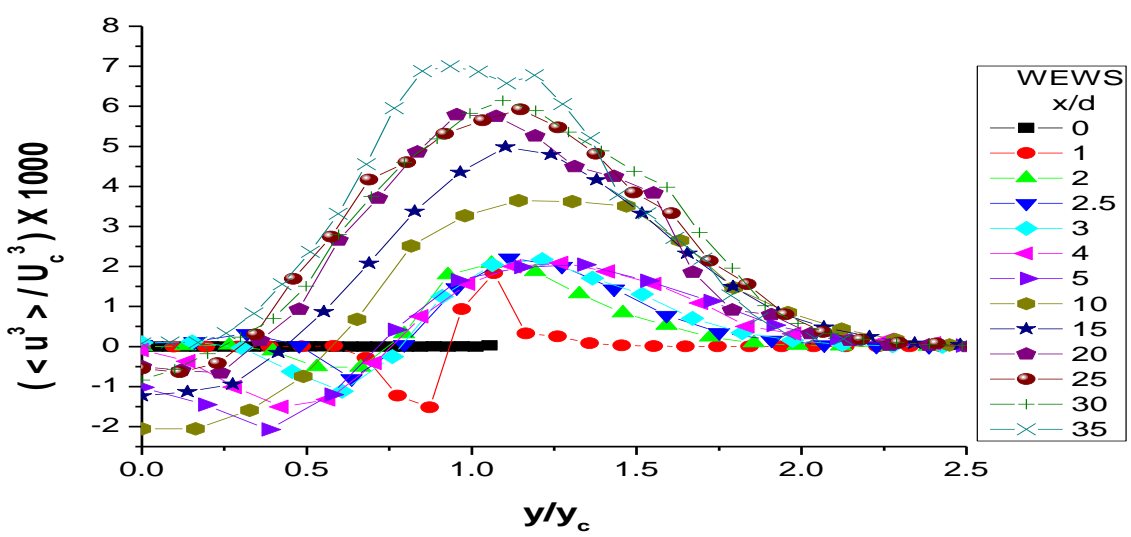

Figure 5.25: Self similarity profiles of triple velocity product $\left\langle u^{3}\right\rangle$ on the central $x y$ plane for all test cases: a) NENS, b) WENS, c) NEWS and d) WEWS (cont'd).

b) Transverse distributions of triple velocity product $\left\langle v^{3}\right\rangle$

Distributions of $\left\langle v^{3}\right\rangle$ normalized by $U_{c}^{3}$ on the central $x y$ plane are shown in Figures 5.26-a, b, c, d , e, f, g, h, j, k, 1, m and $\mathrm{n}$ for downstream locations $x / D=0,1,2,2.5,3$, $4,5,10,15,20,25,30$ and 35 respectively. Close to zero values are observed at positions neighbouring to those where $v^{\prime}$ attains maximum values. From these points towards the centre of the jet, values decrease reaching a negative minimum or zero, close to the inward limit of the shear layer.

In the early stages of jet development $\left\langle v^{3}\right\rangle$ is increasing closer to the centreline, attaining zero values in the potential core. Further downstream $(15 D$ and $30 D)$ the decreasing distributions reach zero on the jet axis (the symmetry properties of this variable enforce a profile symmetric around $(0,0)$ point and zero values on the central 
axis). From the location of maximum $v^{\prime}$, the $\left\langle v^{3}\right\rangle$ values increase towards the edge of the jet reaching a positive maximum outside $y / y_{c}=1$, and decreasing to zero at larger distances from the centreline. Peak values generally increase with $x / D$. The observed similarities and differences between the results of the four configurations follow the trends discussed for the turbulent velocities.

The presence of the endplate has an insignificant effect when the sidewalls have not been implemented and the measurements for NENS and WENS jets are very close at all stations. In the results of the configurations comprising sidewalls, the endplate has a clear effect, and the nondimensional streamwise turbulent velocity attains always higher values in its presence (comparing WEWS with NEWS). The self-preservation of $\left\langle v^{3}\right\rangle$ for all configurations is shown in Figure 27.

a)

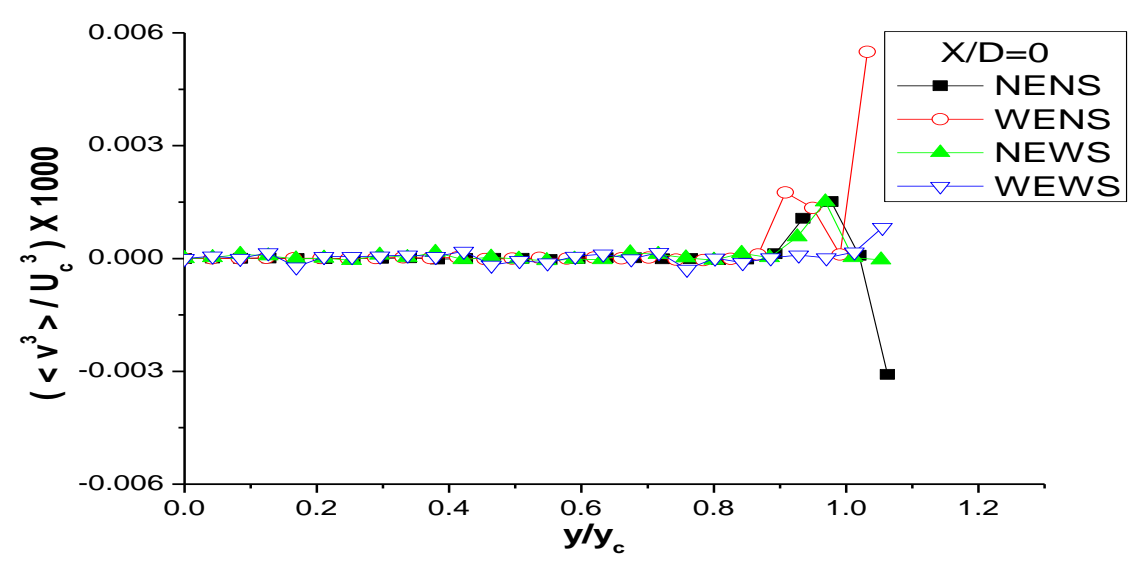

b)

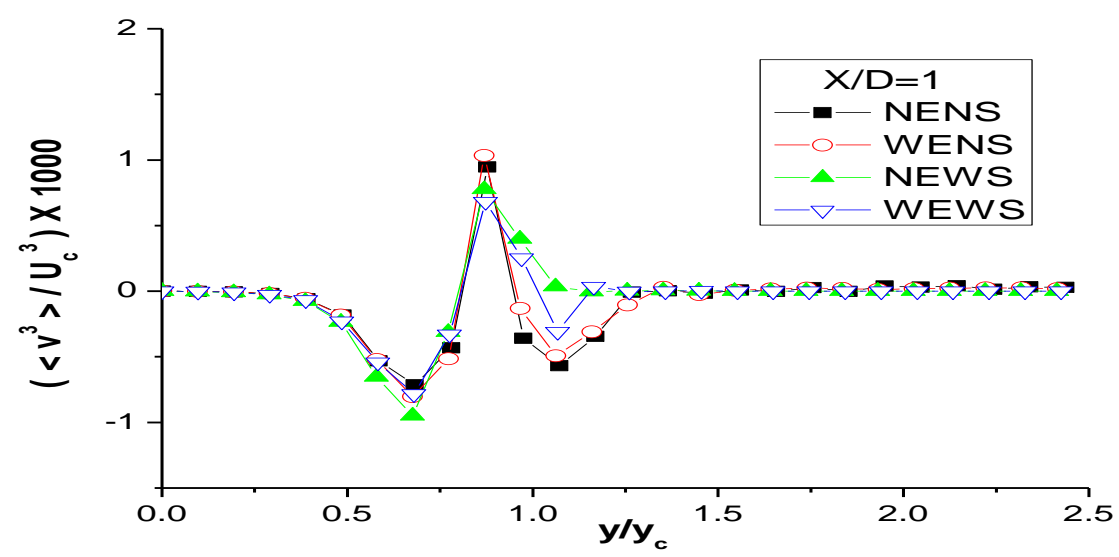

Figure 5.26: Profiles of triple velocity product of $\left\langle v^{3}\right\rangle$ on the central $x y$ plane at different downstream locations. 
c)

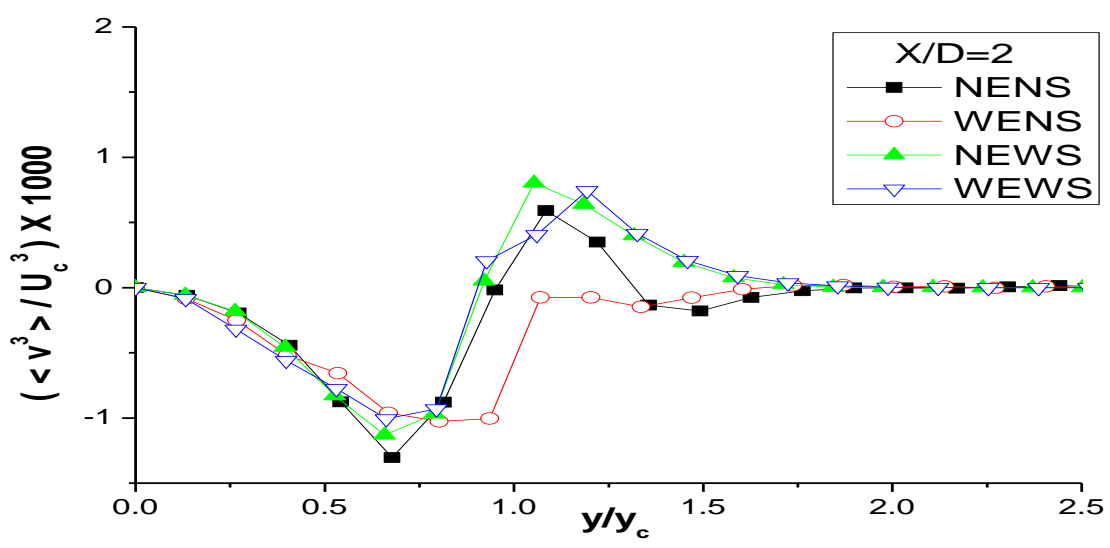

d)

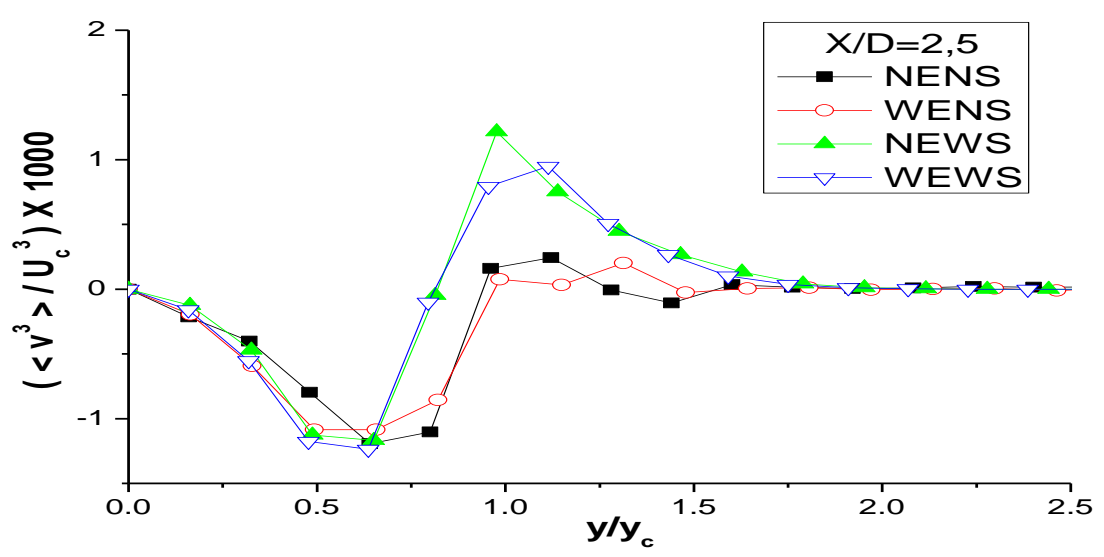

e)

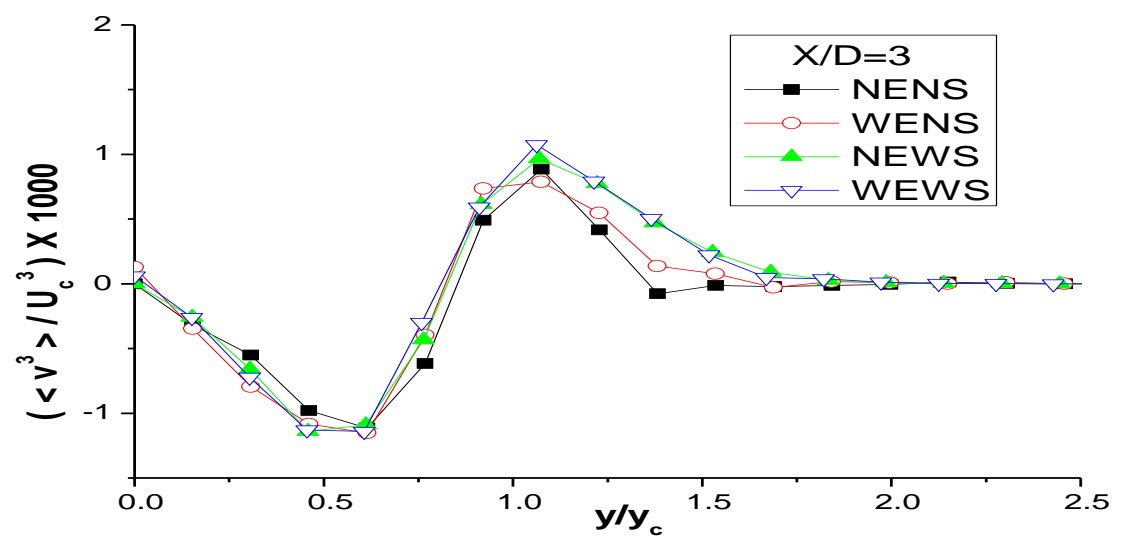

Figure 5.26: Profiles of triple velocity product of $\left\langle v^{3}\right\rangle$ on the central $x y$ plane at different downstream locations (cont'd). 
f)

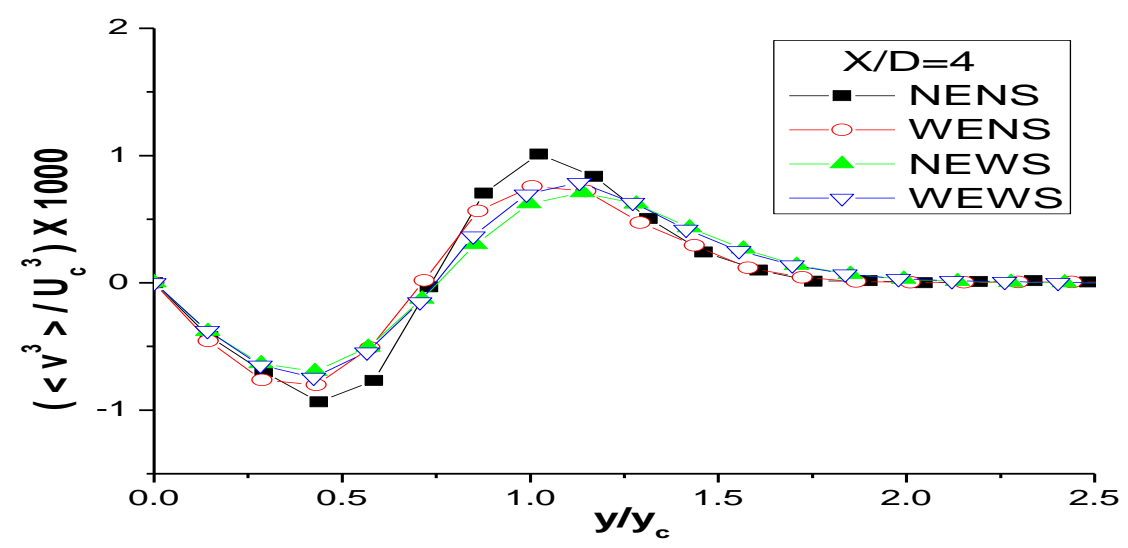

g)

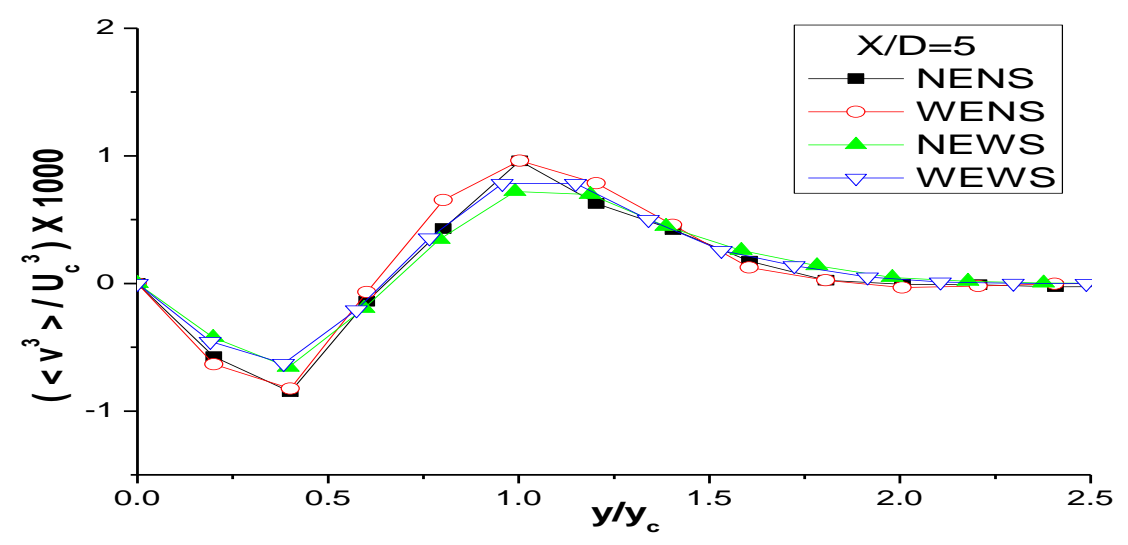

h)

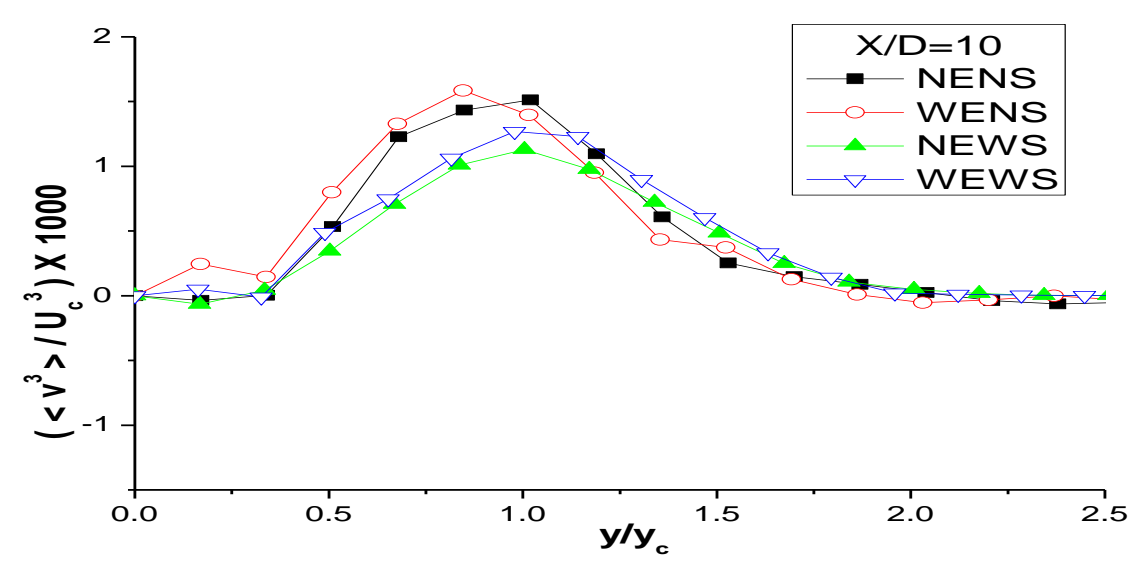

Figure 5.26: Profiles of triple velocity product of $\left\langle v^{3}\right\rangle$ on the central $x y$ plane at different downstream locations (cont'd). 
j)

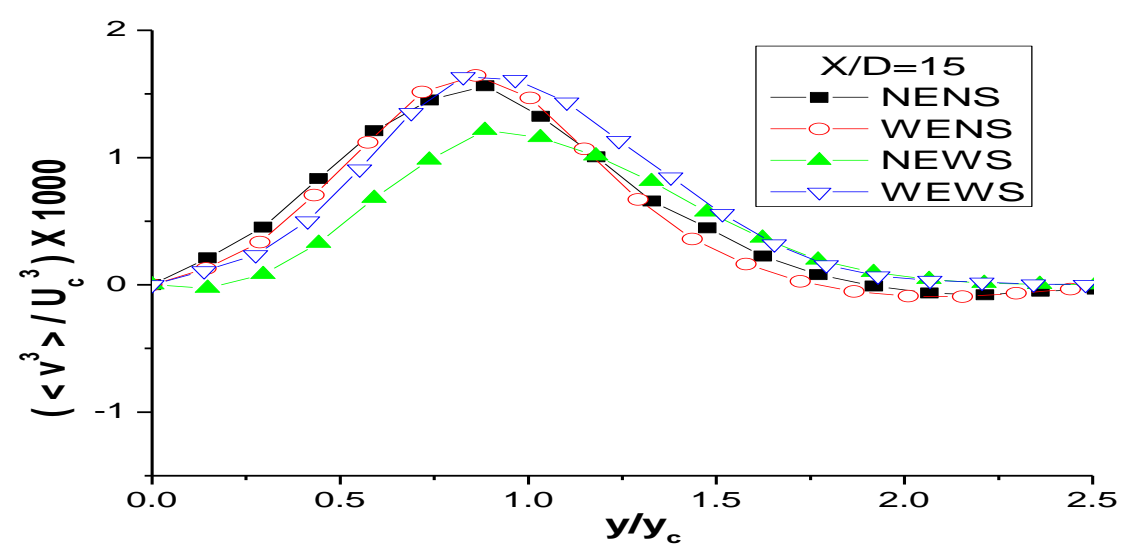

k)

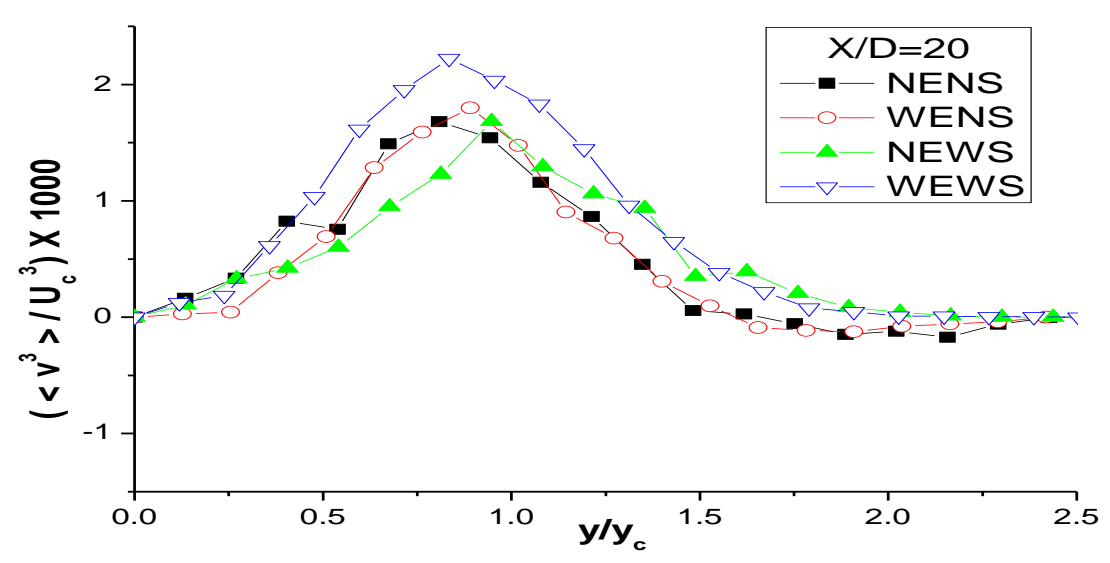

1)

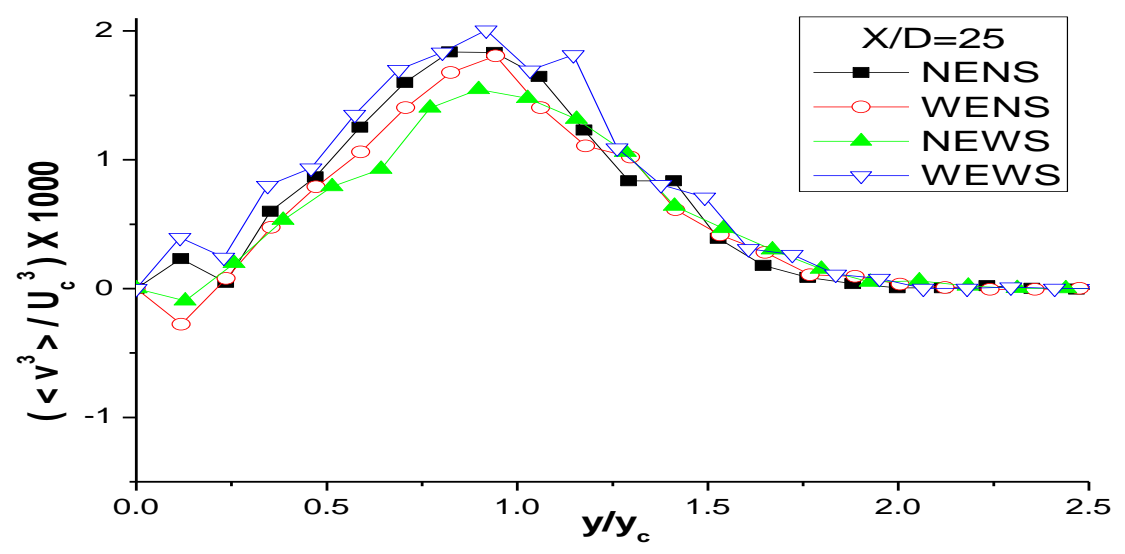

Figure 5.26: Profiles of triple velocity product of $\left\langle v^{3}\right\rangle$ on the central $x y$ plane at different downstream locations (cont'd). 
m)

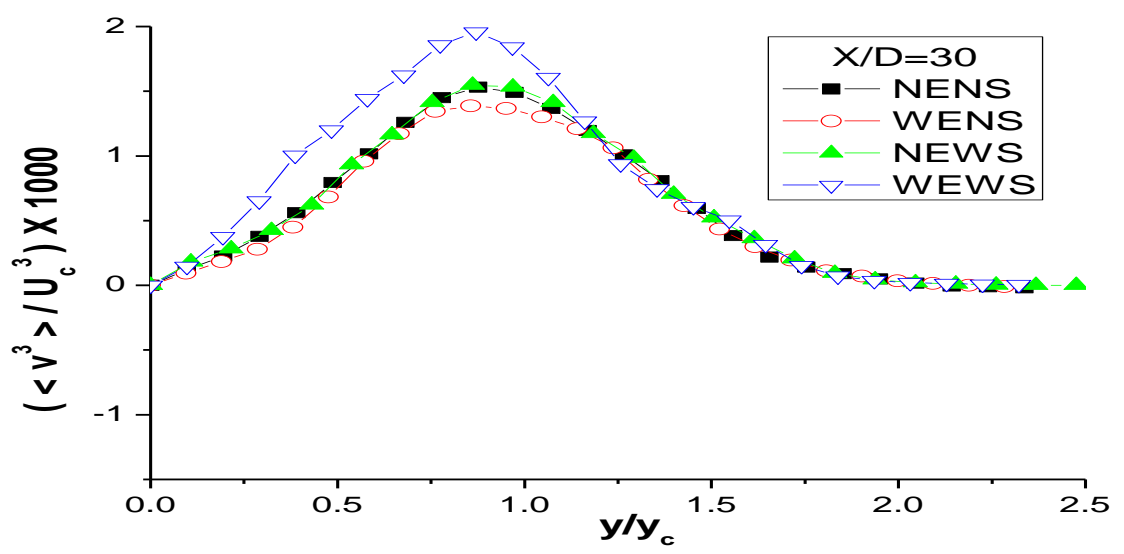

n)

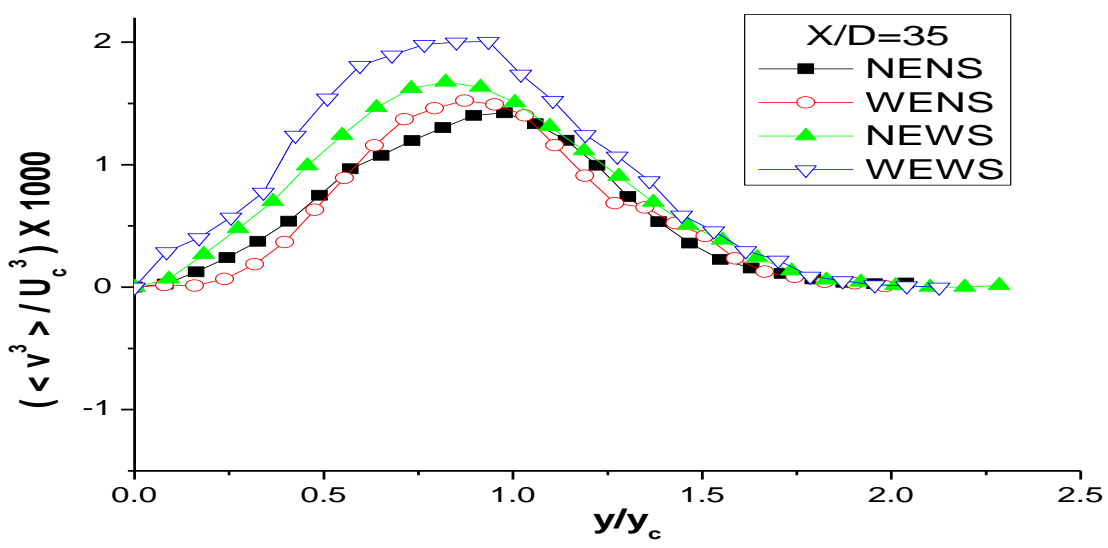

Figure 5.26: Profiles of triple velocity product of $\left\langle v^{3}\right\rangle$ on the central $x y$ plane at different downstream locations (cont'd).

a)

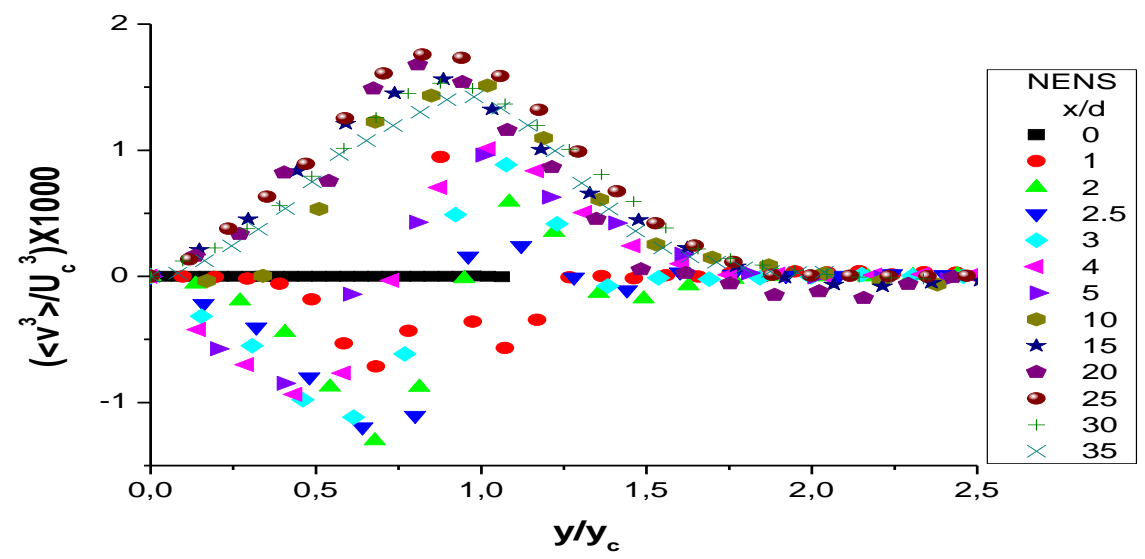

Figure 5.27: Self similarity profiles of triple velocity product $\left\langle v^{3}\right\rangle$ on the central $x y$ plane for all test cases: a) NENS, b) WENS, c) NEWS and d) WEWS. 
b)

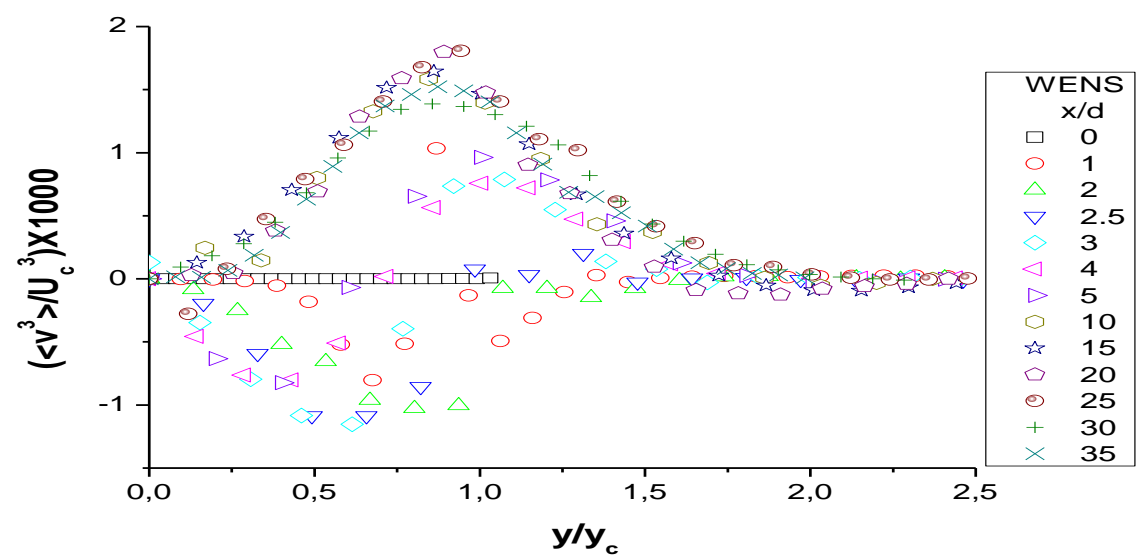

c)

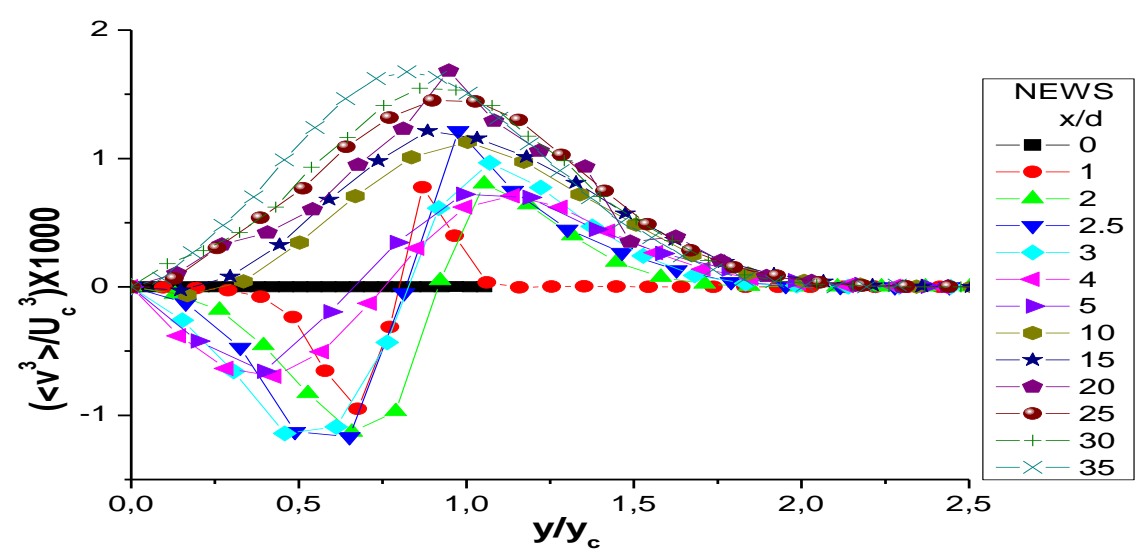

d)

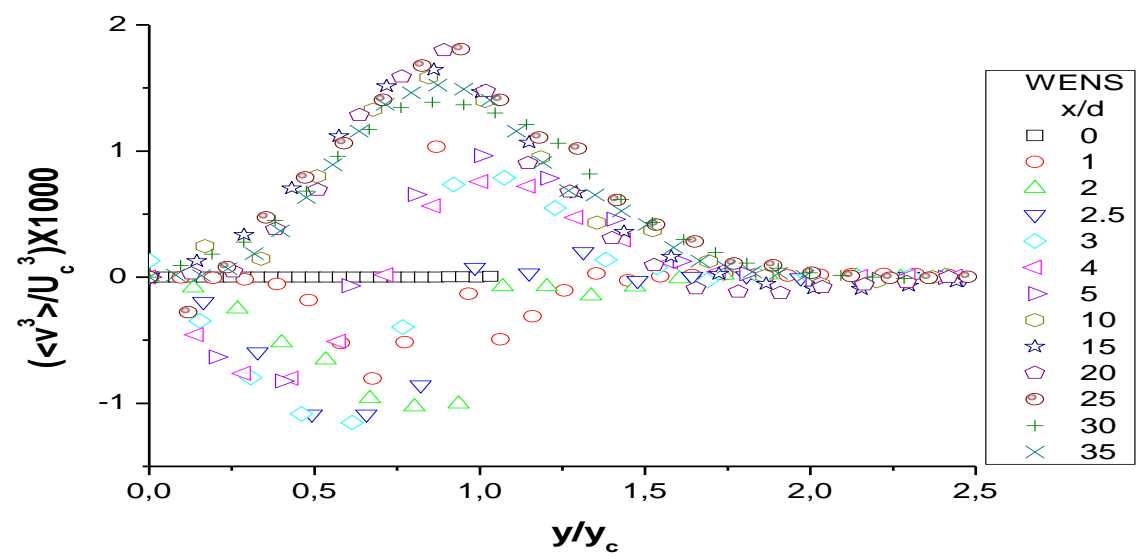

Figure 5.27: Self similarity profiles of triple velocity product $\left\langle v^{3}\right\rangle$ on the central $x y$ plane for all test cases: a) NENS, b) WENS, c) NEWS and d) WEWS (cont'd). 


\section{c) Transverse distributions of triple velocity product $\left\langle u^{2} v\right\rangle$}

The distributions of $\left\langle u^{2} v\right\rangle$ are presented in figures 5.28-a, b, c, d, e, f, g, h, j, k, l, m and $\mathrm{n}$ for downstream locations $x / D=0,1,2,2.5,3,4,5,10,15,20,25,30$ and 35 respectively. They attain almost zero values close to locations of maximum $u^{\prime}$. The trends are very similar to those of $\left\langle u^{3}\right\rangle$ with two main differences. Values are significantly lower and the symmetry properties of this variable always enforce zero values on the central axis. Again the presence or absence of the endplate on jet without sidewalls has insignificant effect as the two distributions of NENS and WENS collapse at downstream locations. Whereas significant effect of the sidewalls is observed which is different when the endplate is introduced (WEWS).

a)

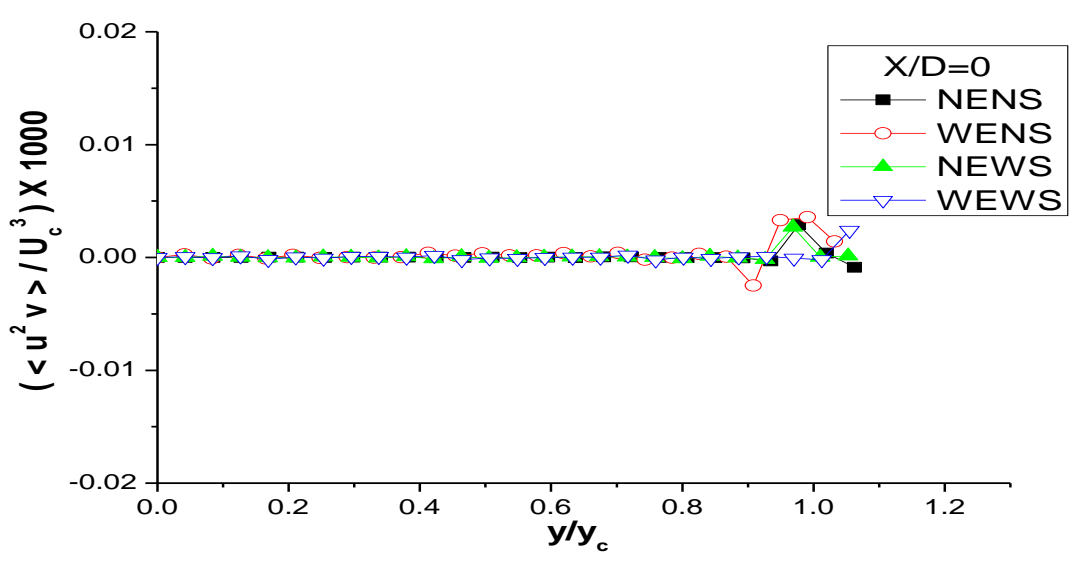

b)

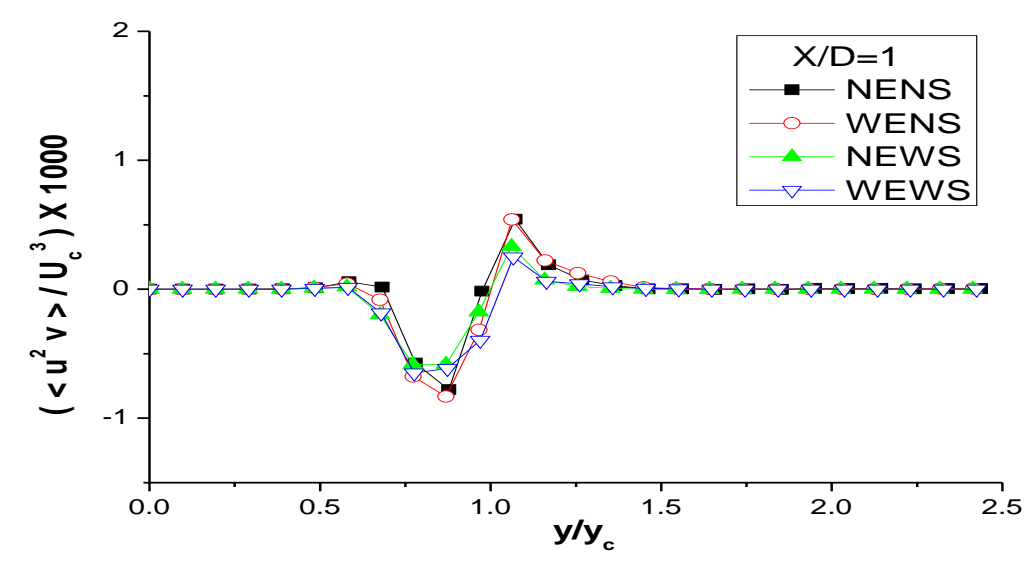

Figure 5.28: Profiles of triple velocity product of $\left\langle u^{2} v\right\rangle$ on the central $x y$ plane at different downstream locations. 
c)

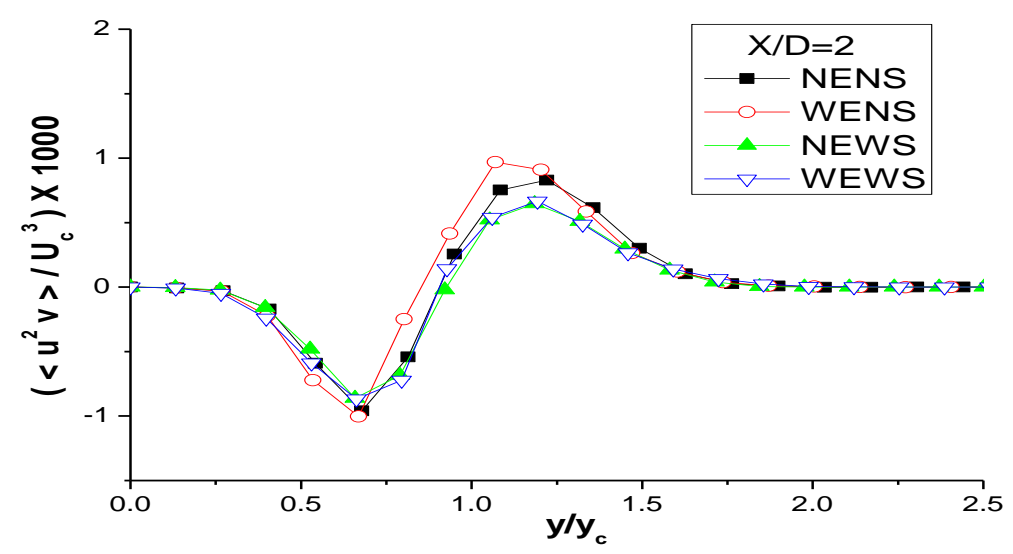

d)

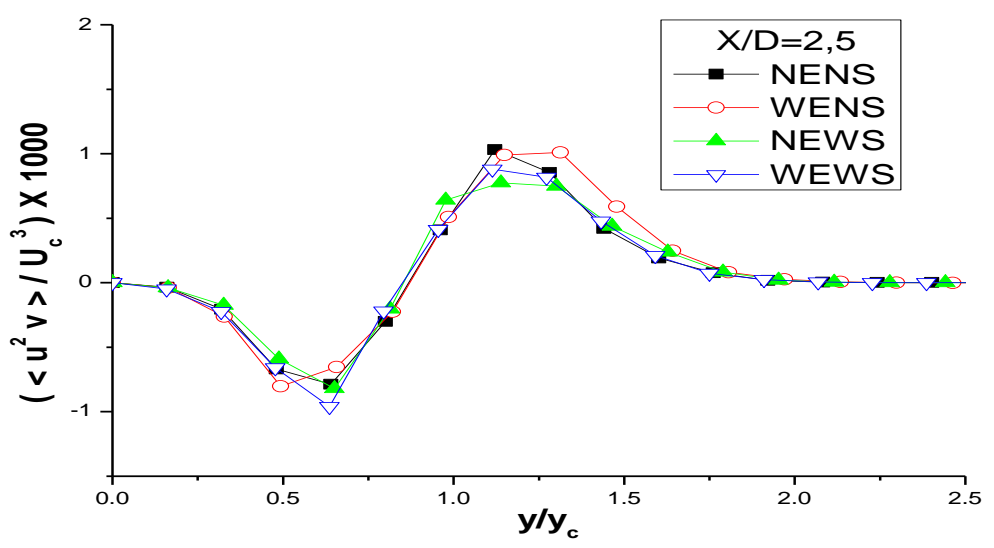

e)

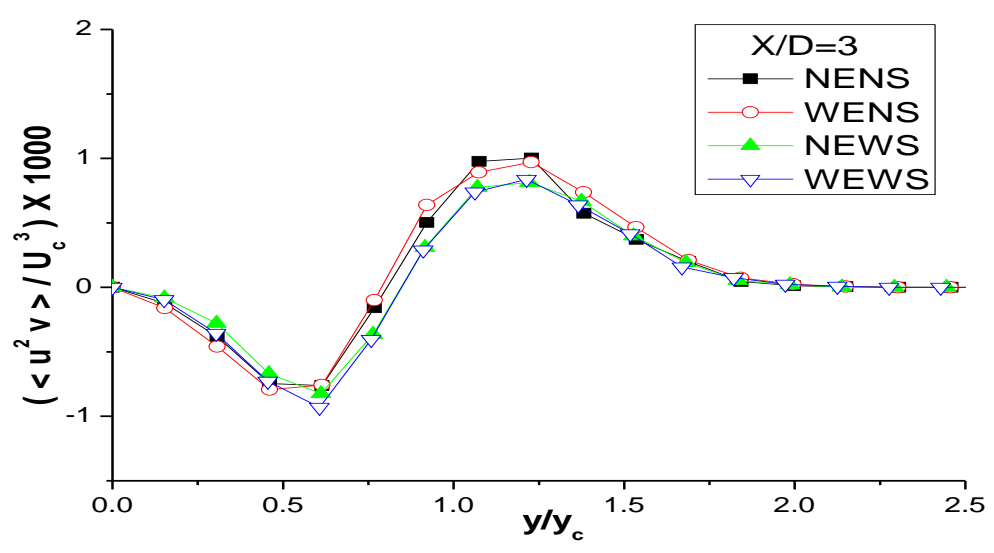

Figure 5.28: Profiles of triple velocity product of $\left\langle u^{2} v\right\rangle$ on the central $x y$ plane at different downstream locations (cont'd). 
f)

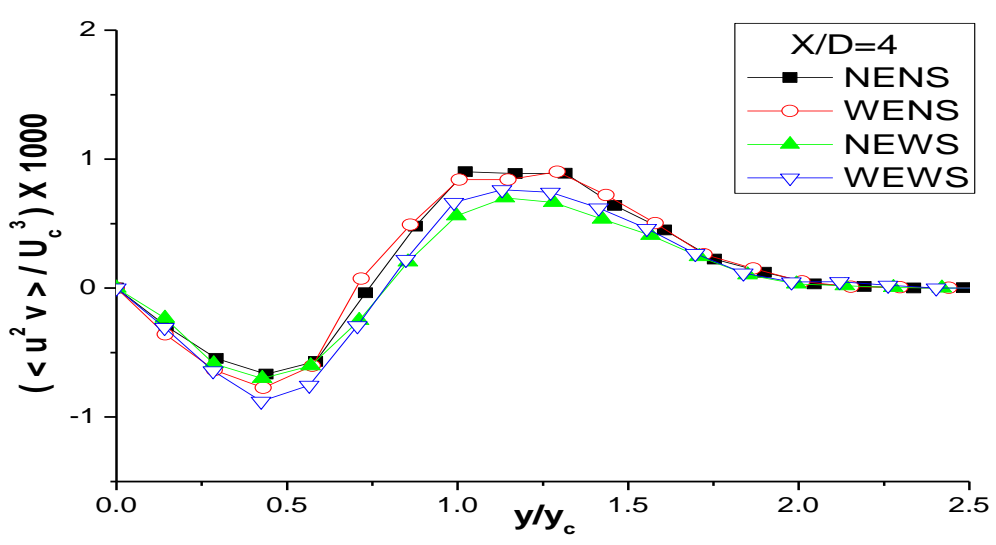

g)

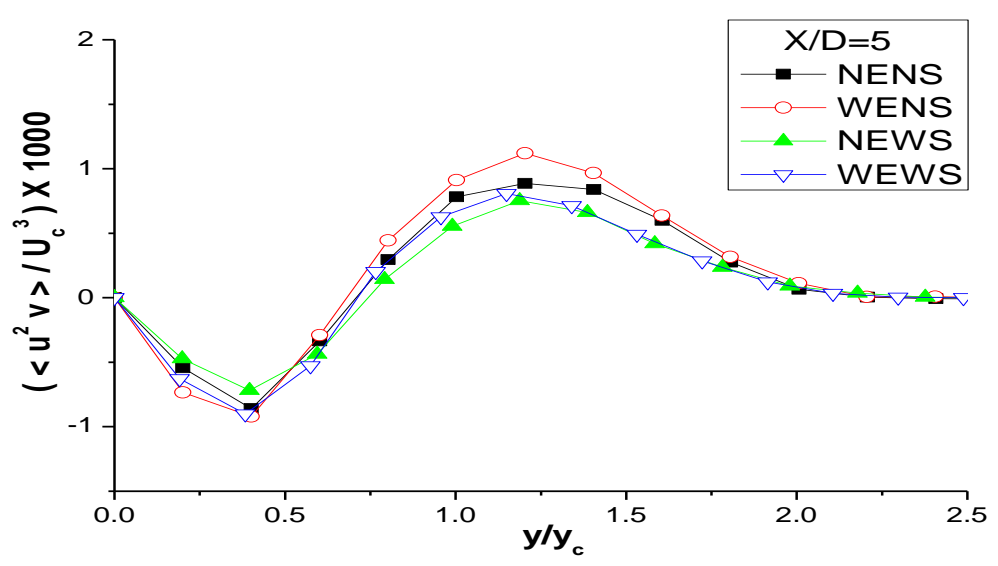

h)

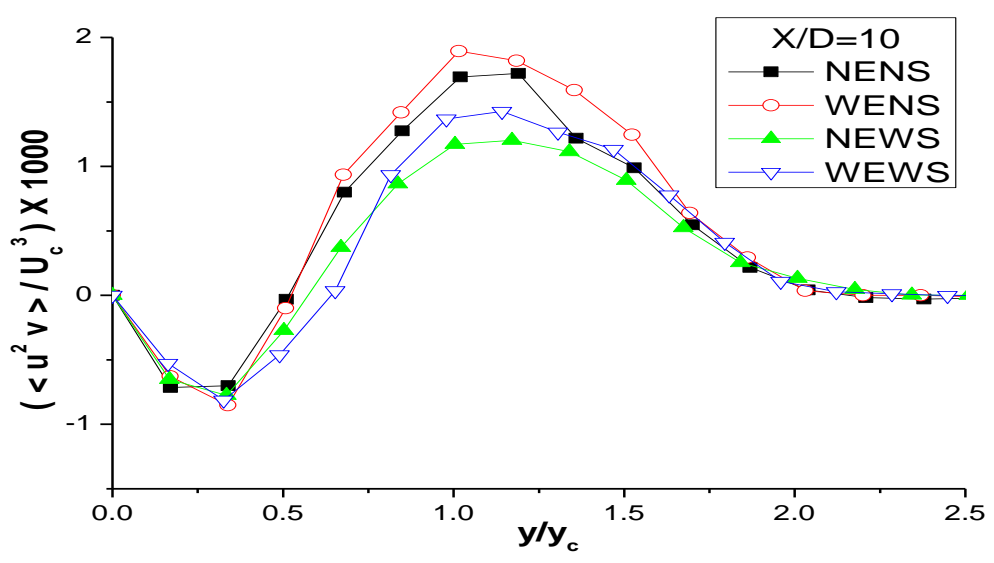

Figure 5.28: Profiles of triple velocity product of $\left\langle u^{2} v\right\rangle$ on the central $x y$ plane at different downstream locations (cont'd). 
j)

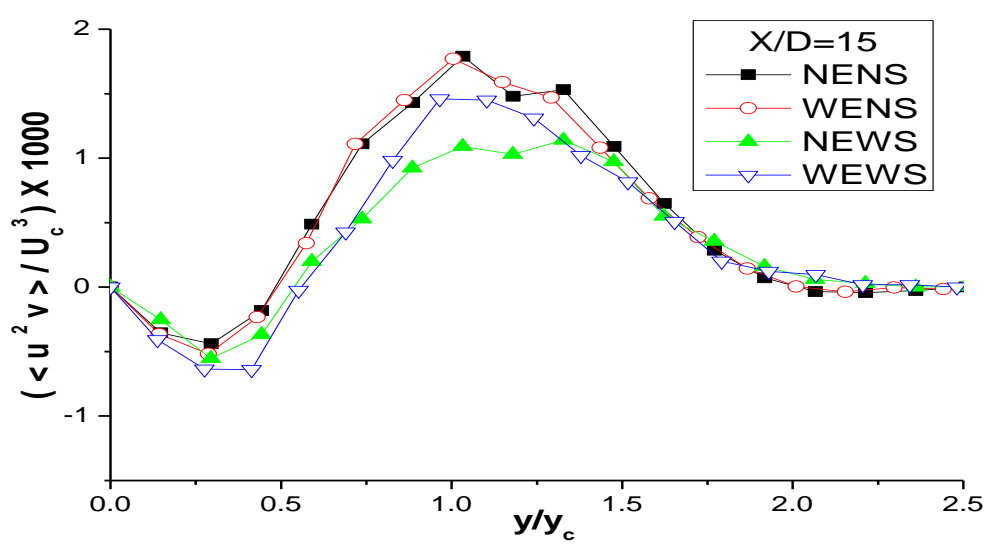

k)

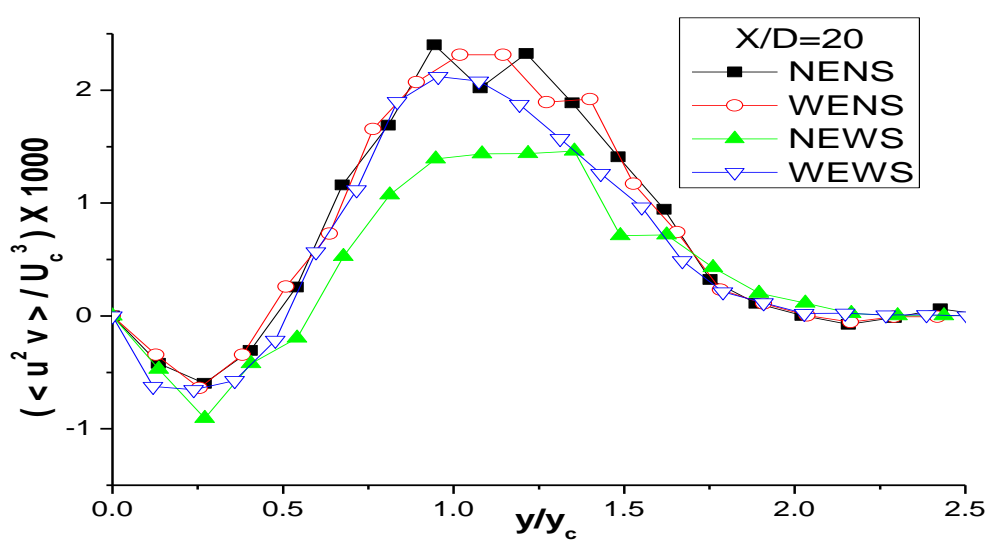

1)

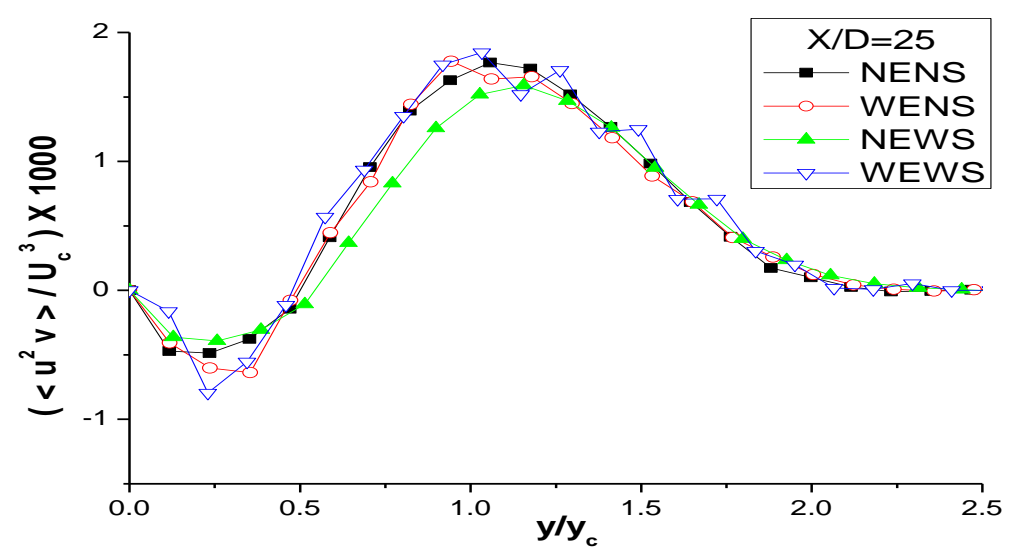

Figure 5.28: Profiles of triple velocity product of $\left\langle u^{2} v\right\rangle$ on the central $x y$ plane at different downstream locations (cont'd). 
$\mathrm{m})$

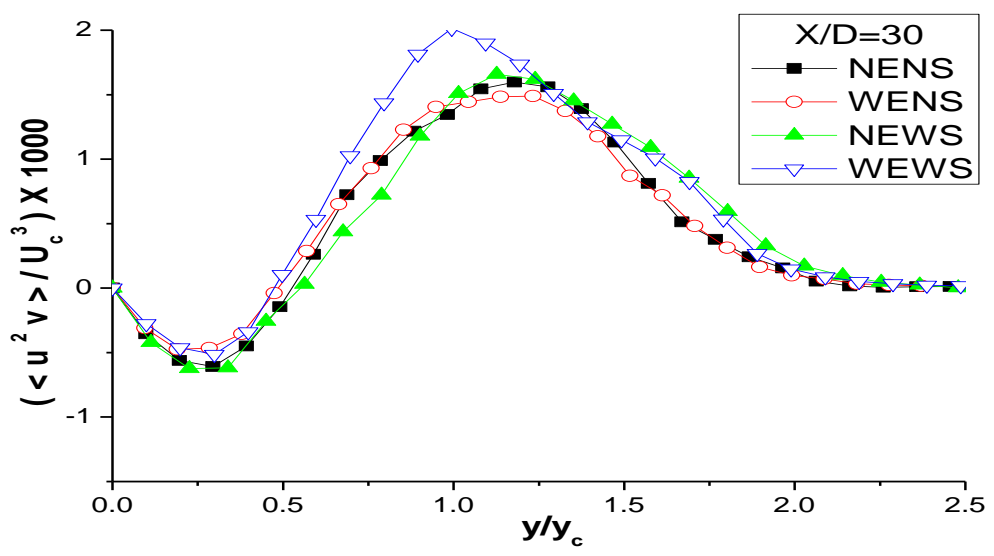

n)

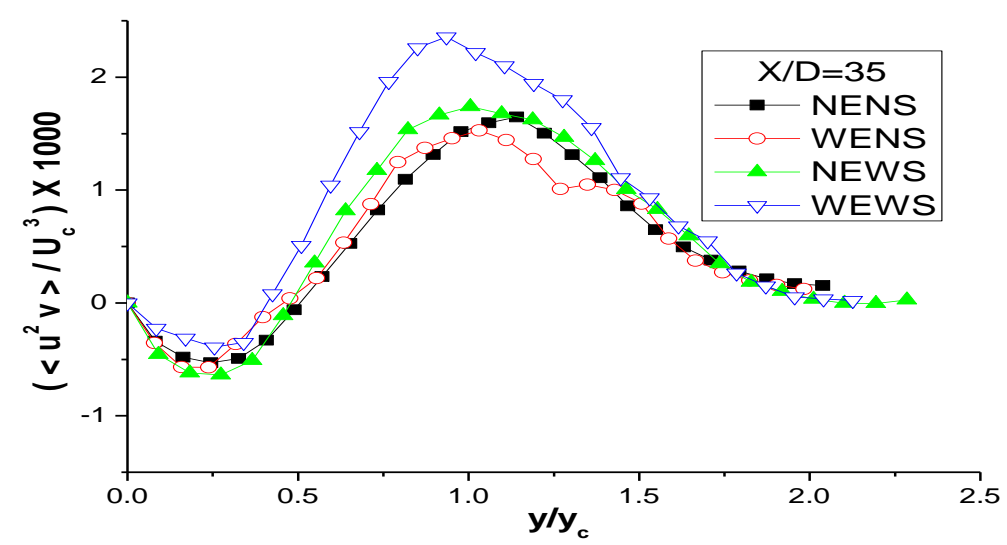

Figure 5.28: Profiles of triple velocity product of $\left\langle u^{2} v\right\rangle$ on the central $x y$ plane at different downstream locations (cont'd).

a)

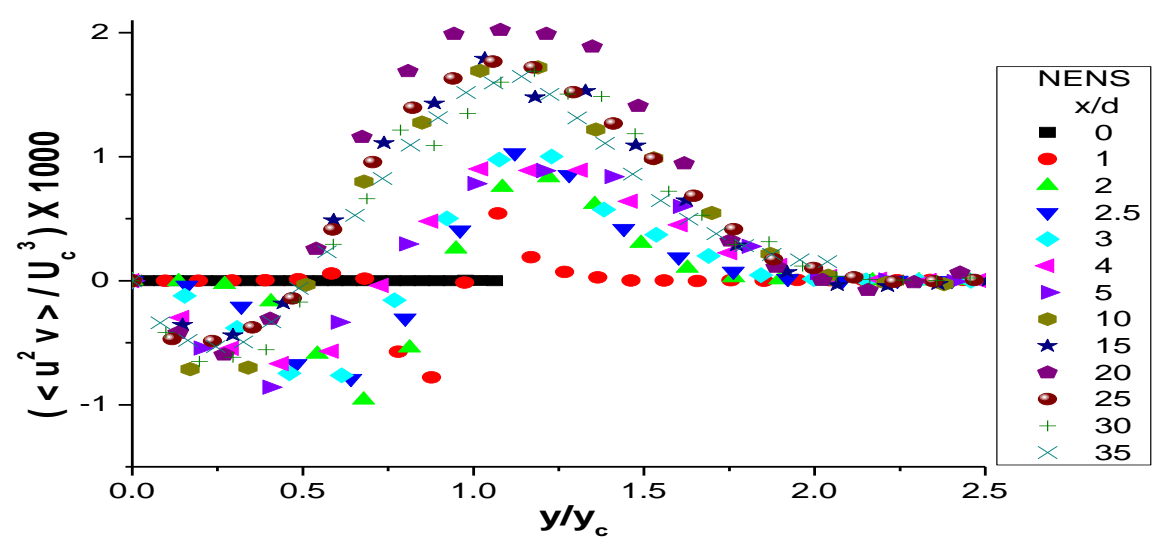

Figure 5.29: Self similarity profiles of triple velocity product $\left\langle u^{2} v\right\rangle$ on the central $x y$ plane for all test cases: a) NENS, b) WENS, c) NEWS and d) WEWS. 
b)

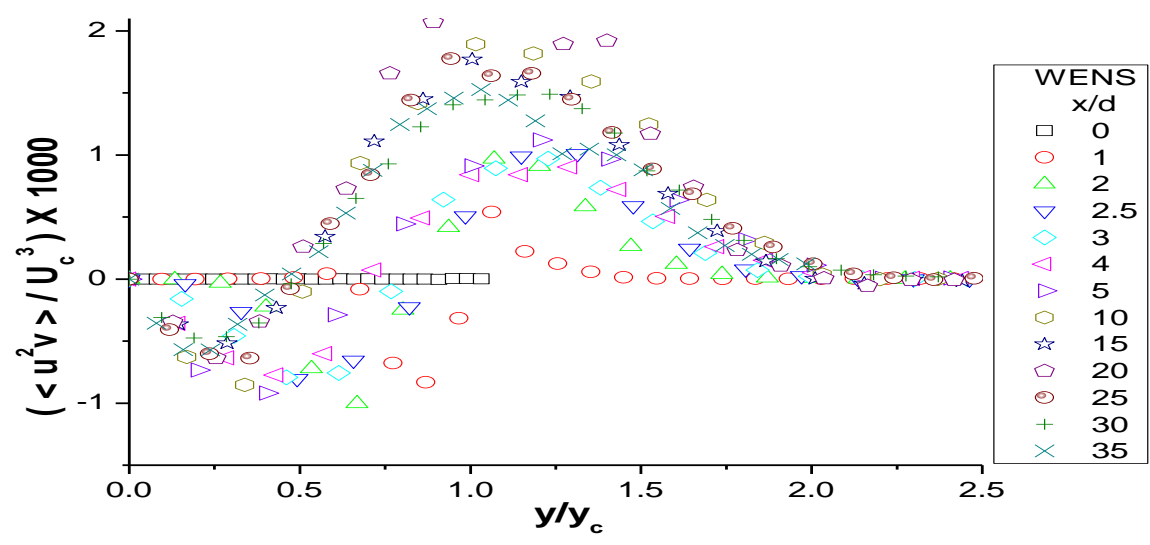

c)

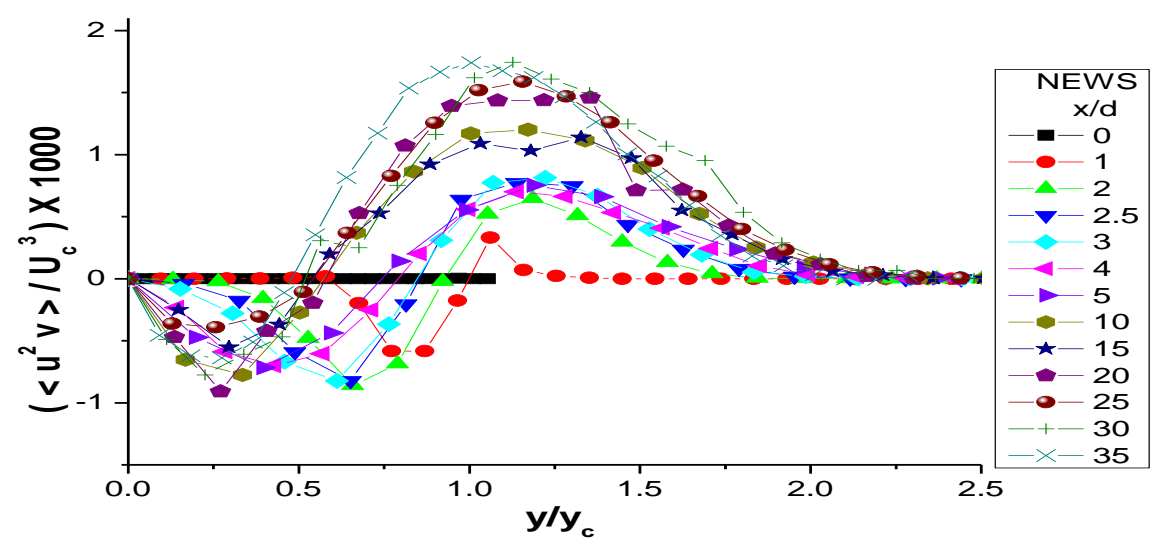

d)

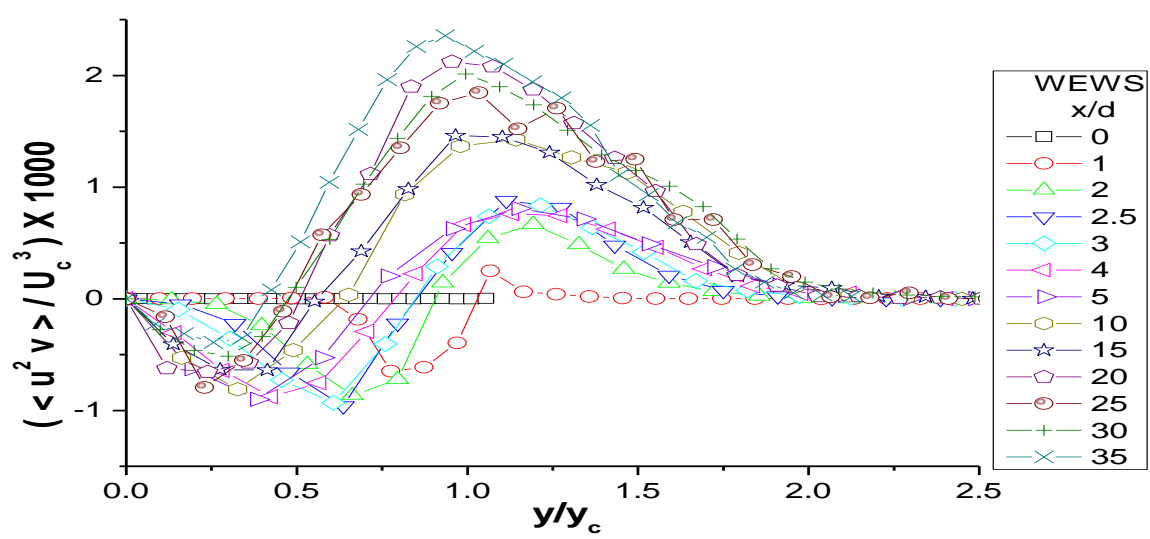

Figure 5.29: Self similarity profiles of triple velocity product $\left\langle u^{2} v\right\rangle$ on the central $x y$ plane for all test cases: a) NENS, b) WENS, c) NEWS and d) WEWS (cont'd). 


\section{d) Transverse distributions of triple velocity product $\left\langle u v^{2}\right\rangle$}

The features of the $\left\langle u v^{2}\right\rangle$ profiles presented in Figure 5.30 are very similar to those of $\left\langle u^{3}\right\rangle$ although amplitudes are significantly lower. The self preservation behaviour of these profiles is shown in Figure 5.31.

a)

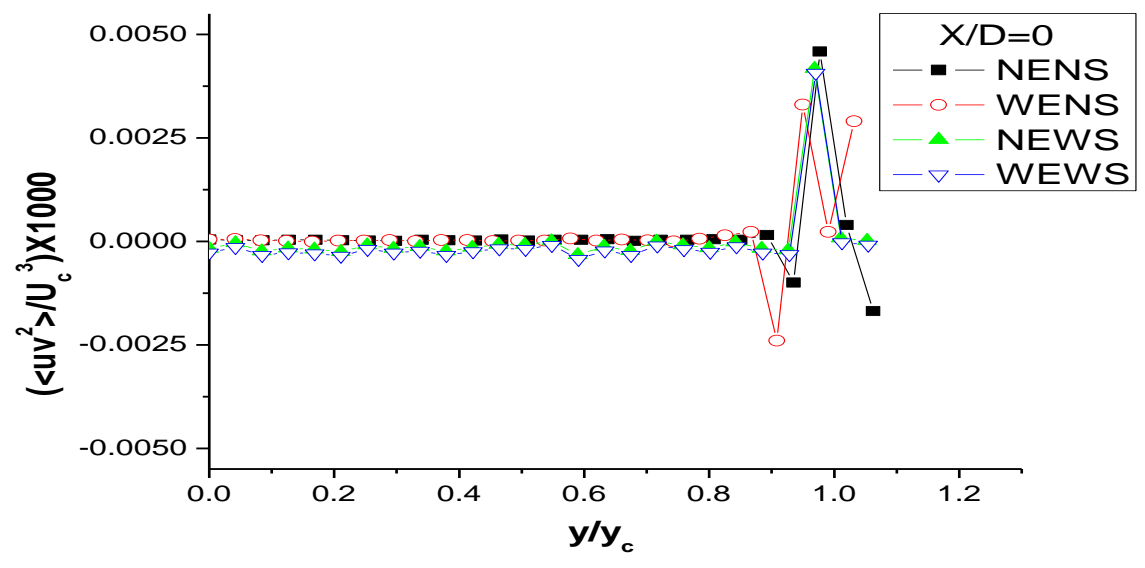

b)

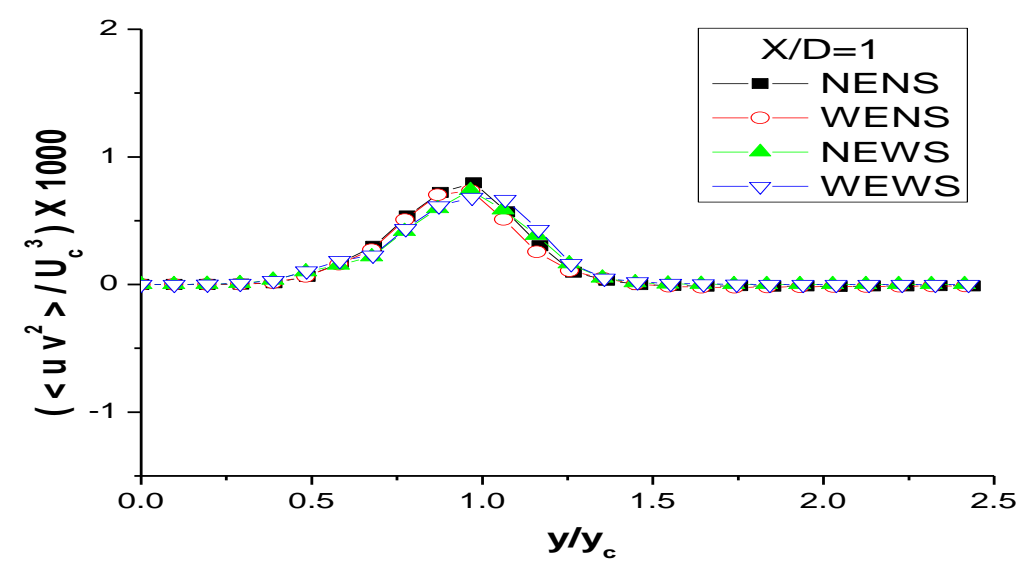

c)

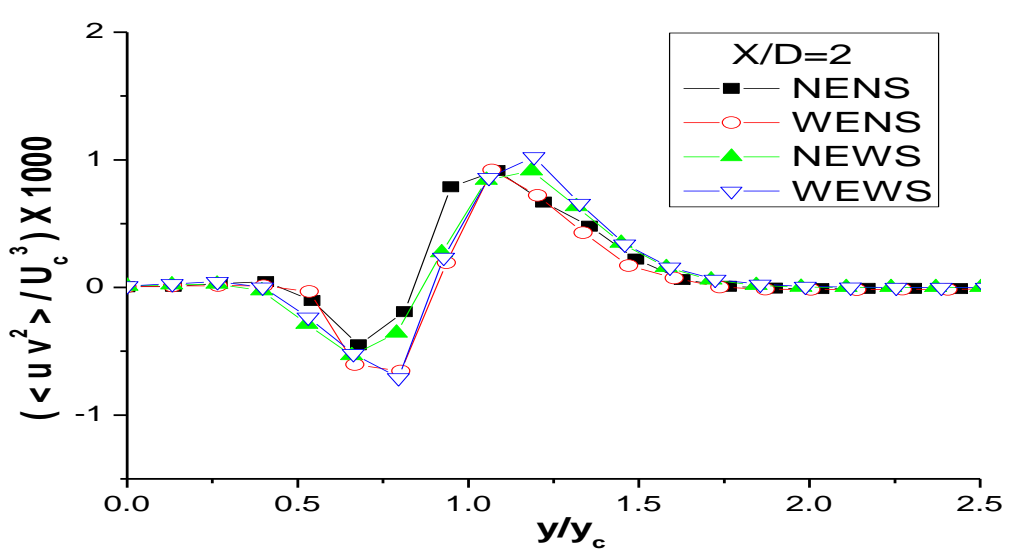

Figure 5.30: Profiles of triple velocity product of $\left\langle u v^{2}\right\rangle$ on the central $x y$ plane at different downstream locations. 
d)

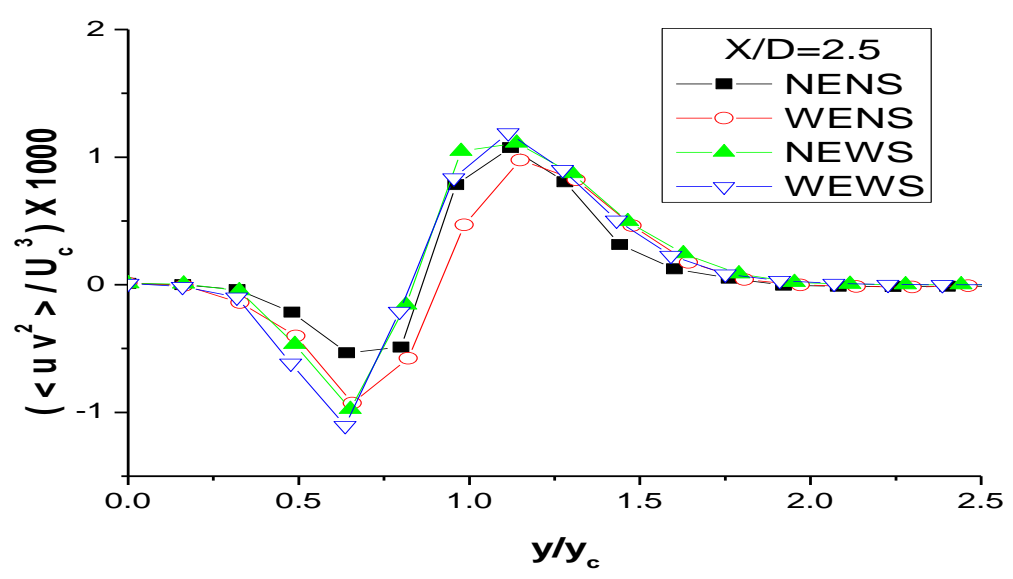

e)

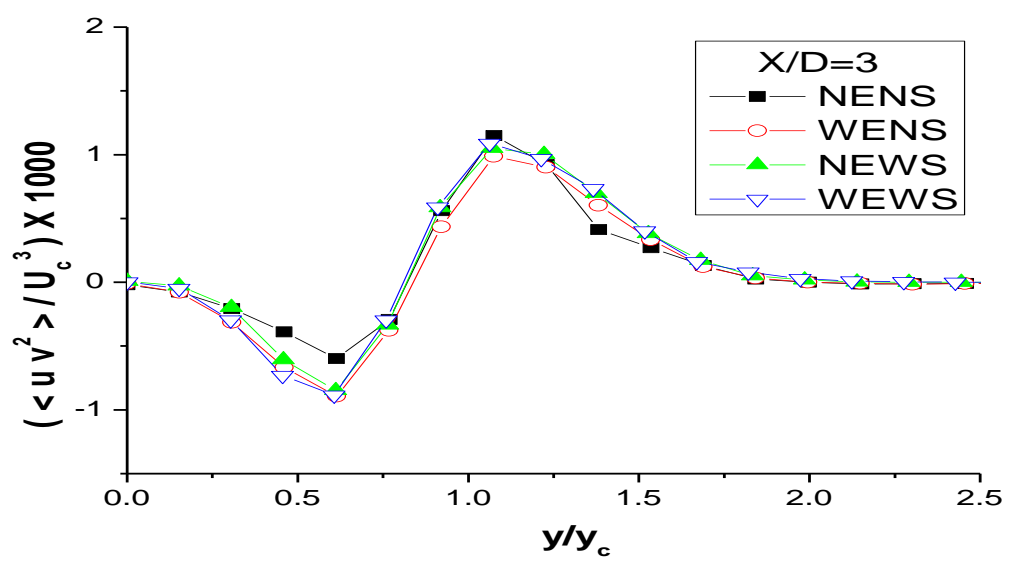

f)

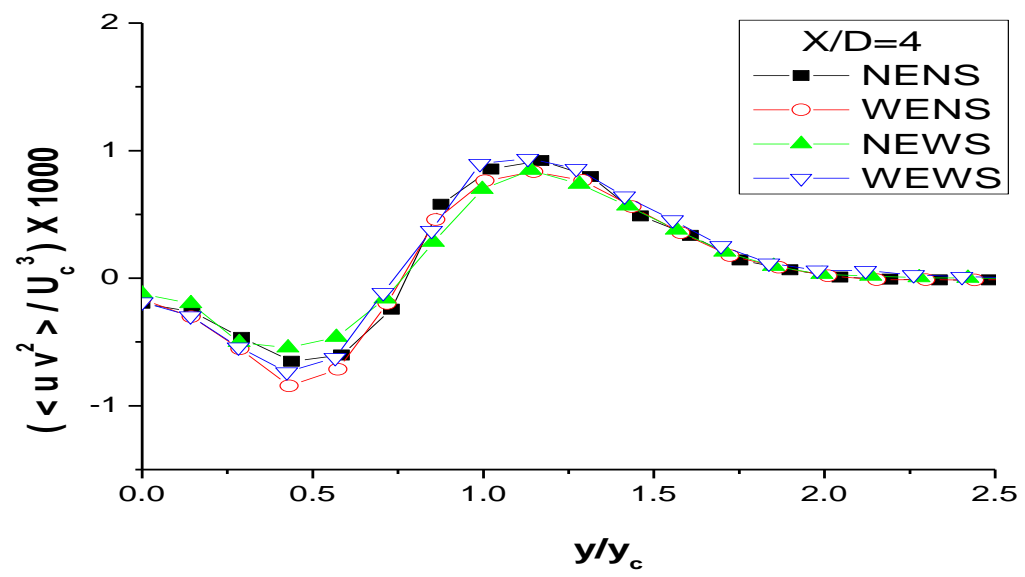

Figure 5.30: Profiles of triple velocity product of $\left\langle u v^{2}\right\rangle$ on the central $x y$ plane at different downstream locations (cont'd). 
g)

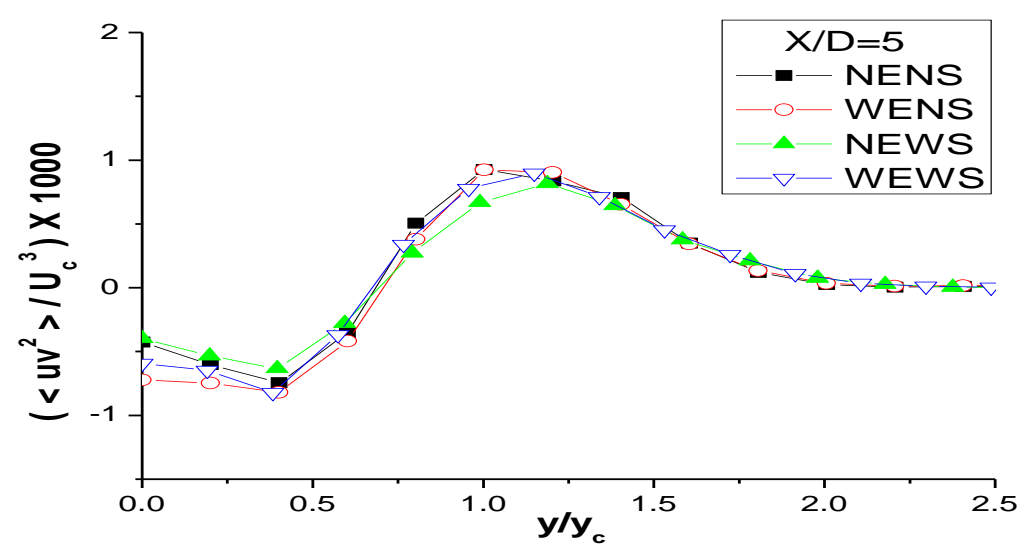

h)

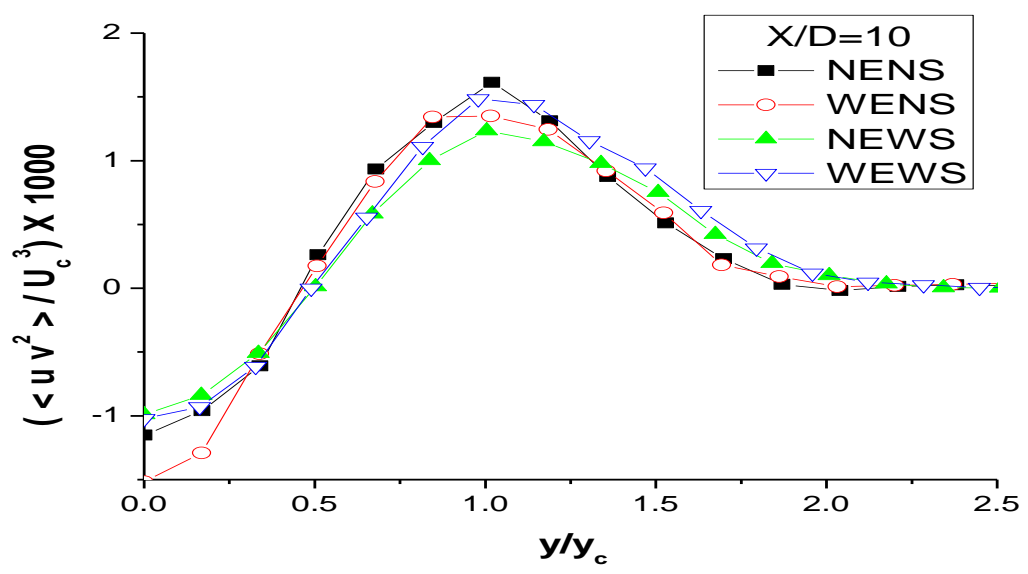

j)

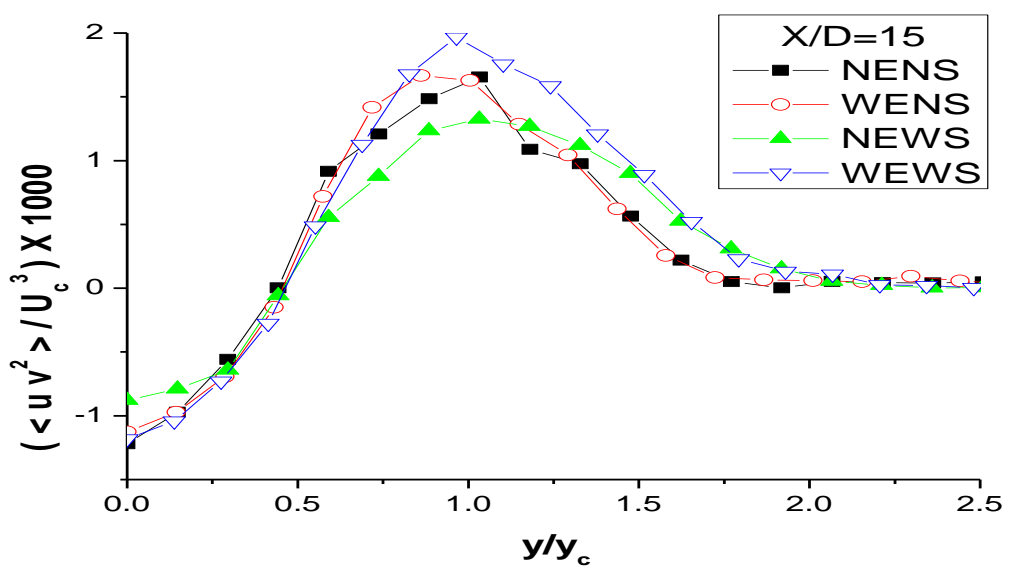

Figure 5.30: Profiles of triple velocity product of $\left\langle u v^{2}\right\rangle$ on the central $x y$ plane at different downstream locations (cont'd). 
k)

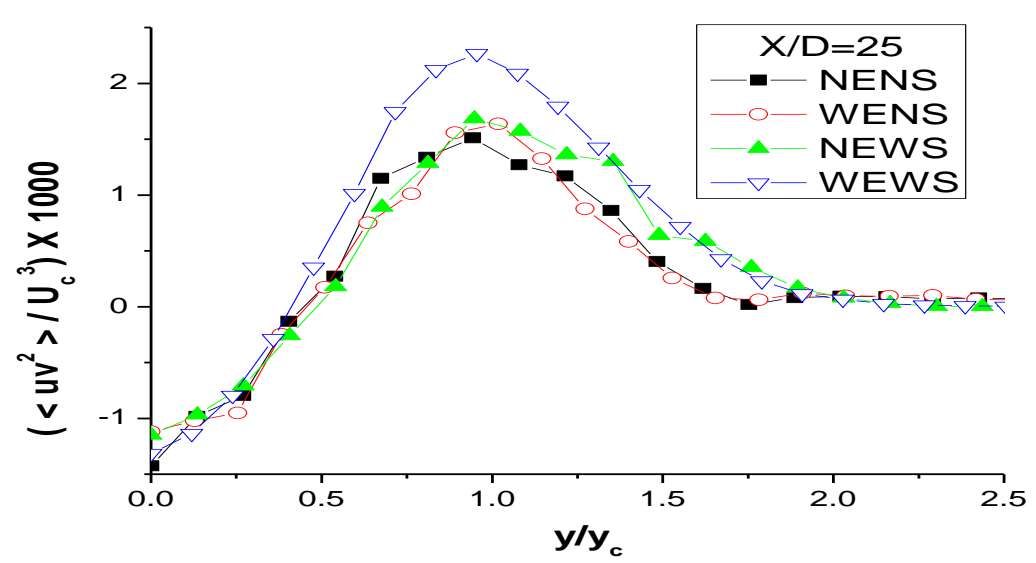

1)

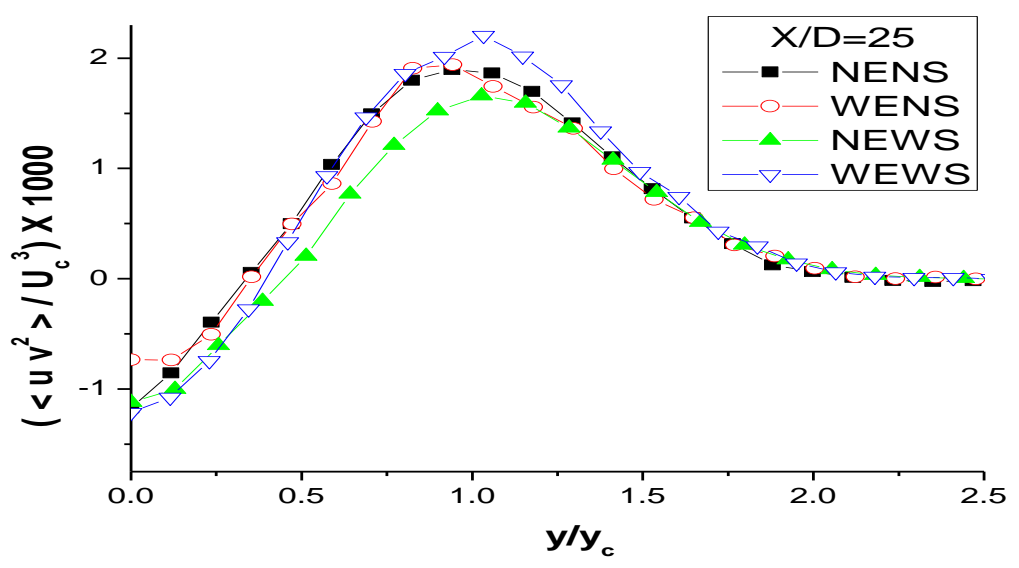

m)

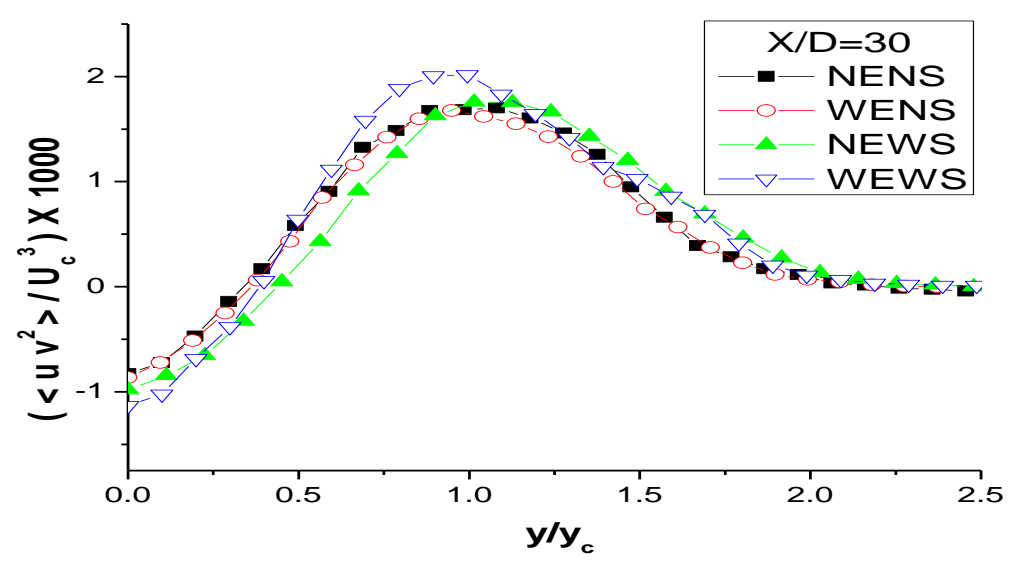

Figure 5.30: Profiles of triple velocity product of $\left\langle u v^{2}\right\rangle$ on the central $x y$ plane at different downstream locations (cont'd). 
n)

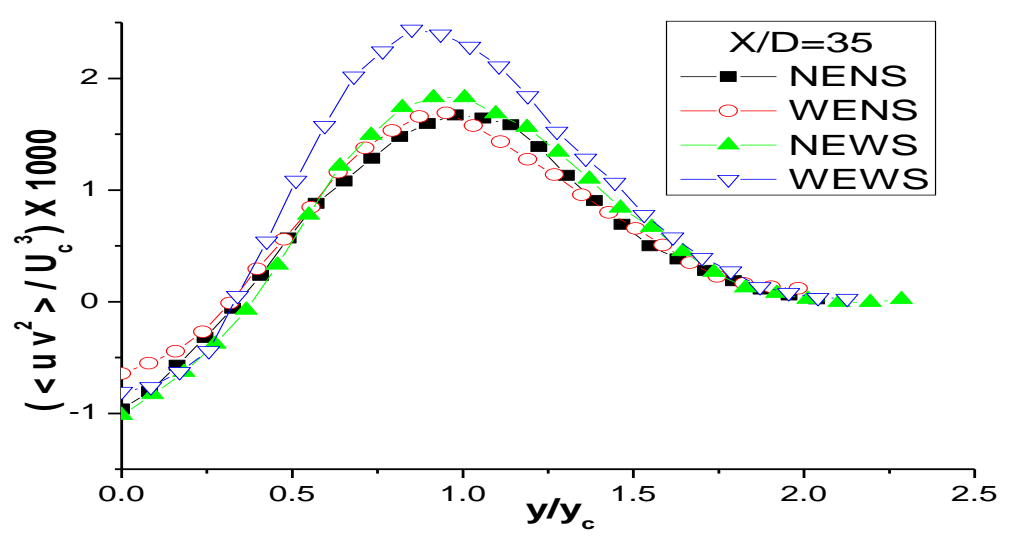

Figure 5.30: Profiles of triple velocity product of $\left\langle u v^{2}\right\rangle$ on the central $x y$ plane at different downstream locations (cont'd).

a)

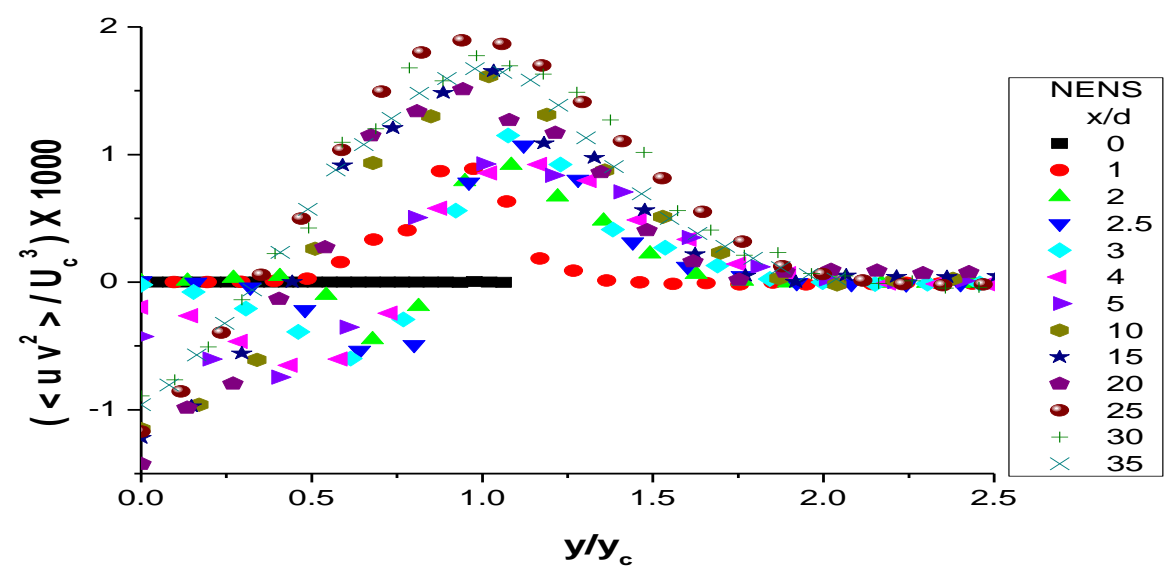

b)

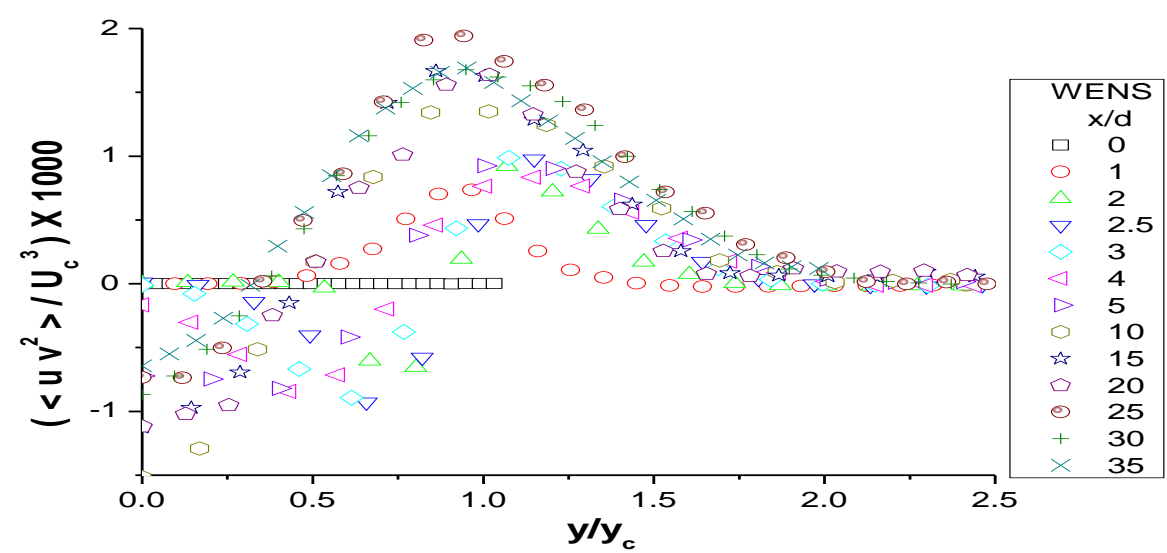

Figure 5.31: Self similarity profiles of triple velocity product $\left\langle u v^{2}\right\rangle$ on the central $x y$ plane for all test cases: a) NENS, b) WENS, c) NEWS and d) WEWS. 
c)

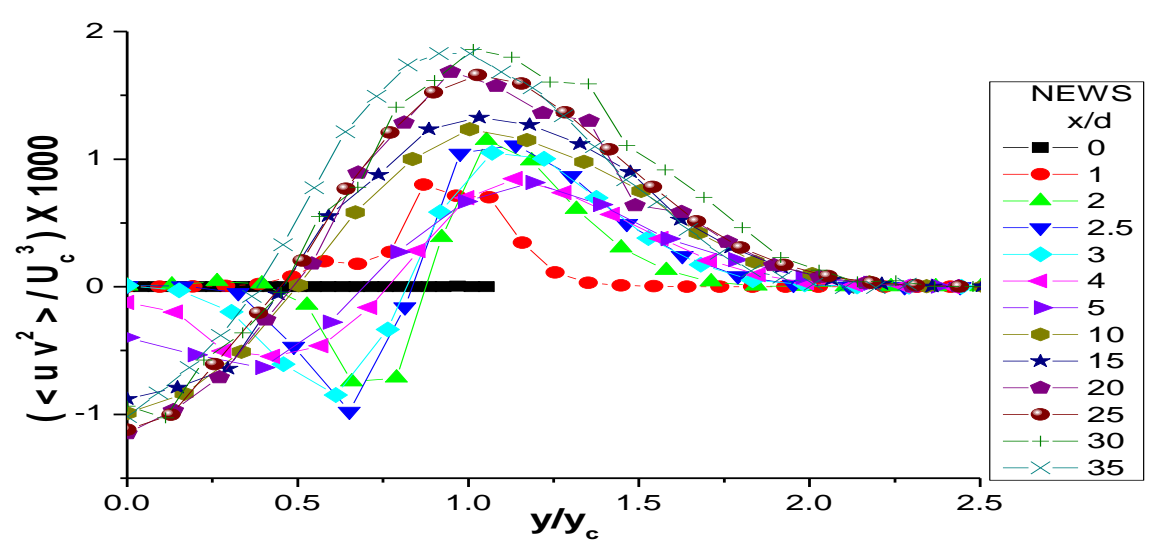

d)

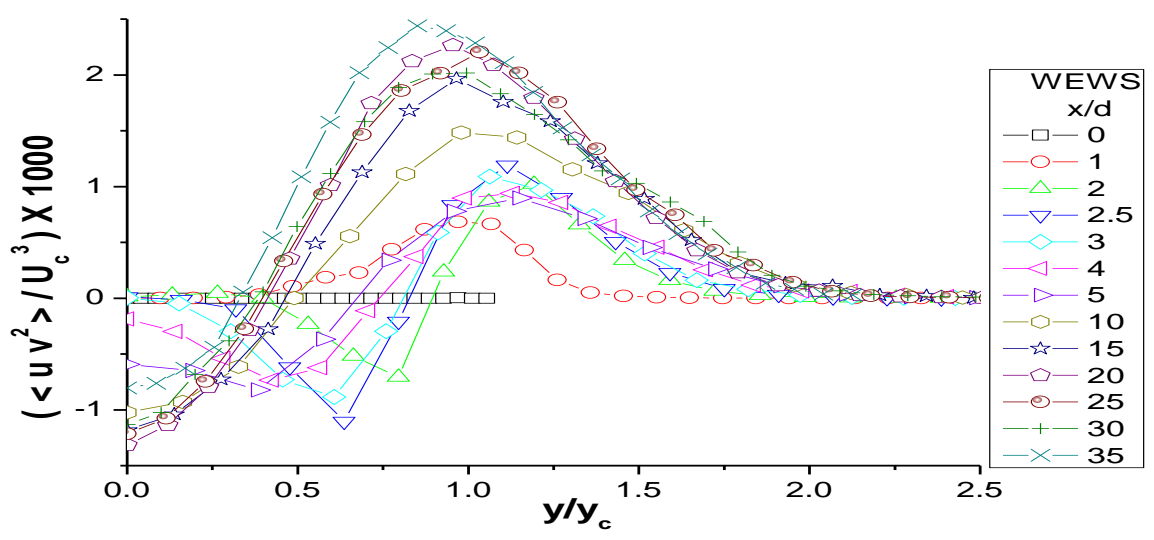

Figure 5.31: Self similarity profiles of triple velocity product $\left\langle u v^{2}\right\rangle$ on the central $x y$ plane for all test cases: a) NENS, b) WENS, c) NEWS and d) WEWS (cont'd).

\subsubsection{Skewness factor}

\section{a) Transverse distributions of skewness factor of the longitudinal velocity}

The transverse distributions of the skewness factor of the longitudinal velocity in the $x y$ plane, i.e. $S_{u}=\left\langle u^{3}\right\rangle / u^{\prime 3}$ are shown in Figures 5.32-a, b, c, d, e, f, g, h, j, k, l, m and $\mathrm{n}$ for downstream locations $x / D=0,1,2,2.5,3,4,5,10,15,20,25,30$ and 35 respectively. $S_{u}$ usually give insight into the flow field which is a measure of the statistical symmetry of $u$ (where $u$ is fluctuating streamwise velocity). As evidenced, the distributions of NENS and WENS are nearly identical at all downstream location indicating the insignificant effect of the endplate when sidewalls are not present. When the sidewalls are present (NEWS, WEWS), sharp peaks are found at the outer 
region of the jets. These peaks which are produced indicate to presence of 2-d rollers which are formed in jets with sidewalls. Figure 5.33 demonstrates the selfpreservation behaviour of $S_{u}$ for the four test cases which indicates similar trends as other turbulent quantities.

a)

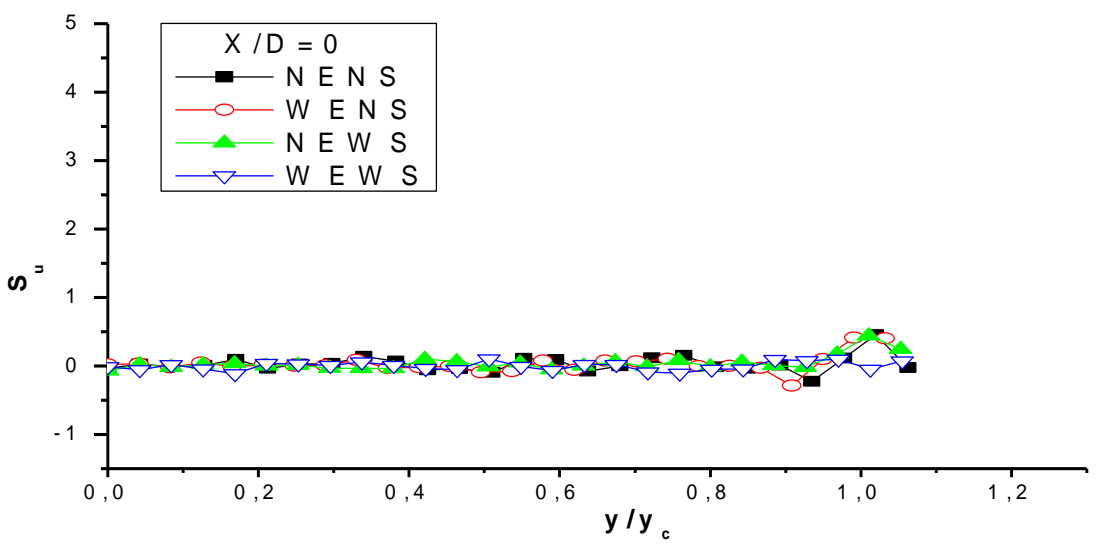

b)

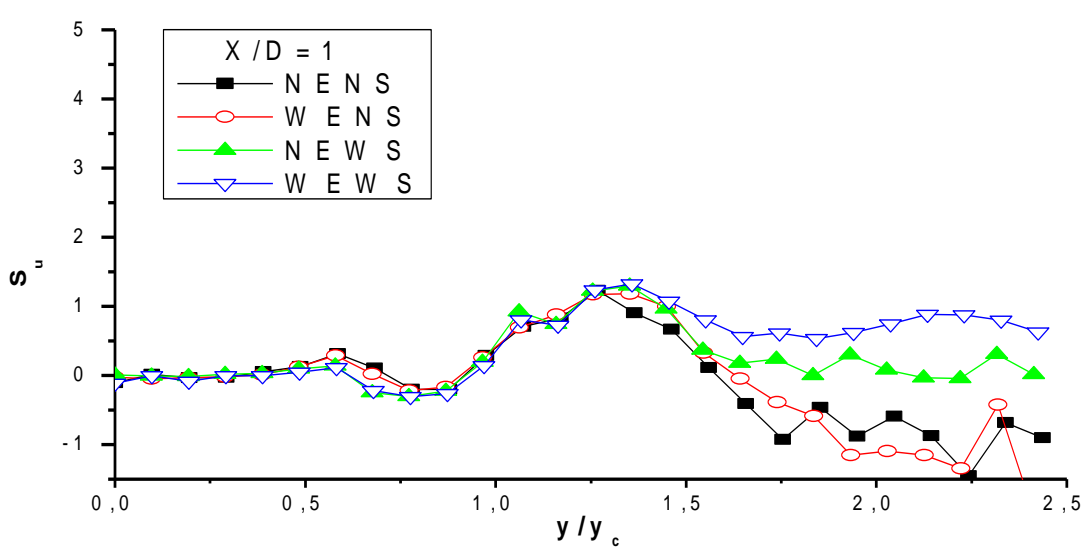

c)

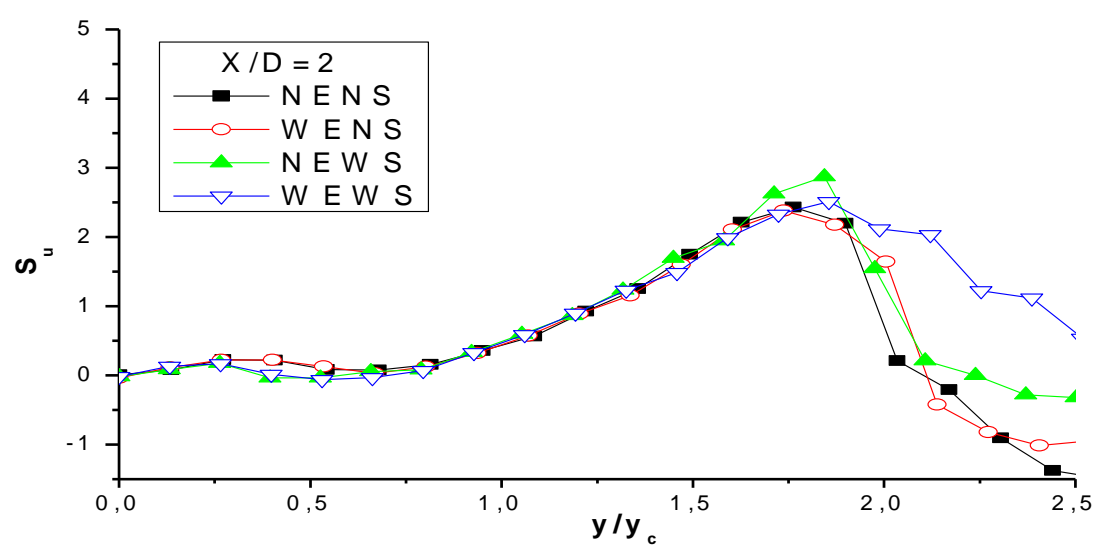

Figure 5.32: Skewness profiles of the longitudinal velocity on the central $x y$ plane at different downstream locations. 
d)

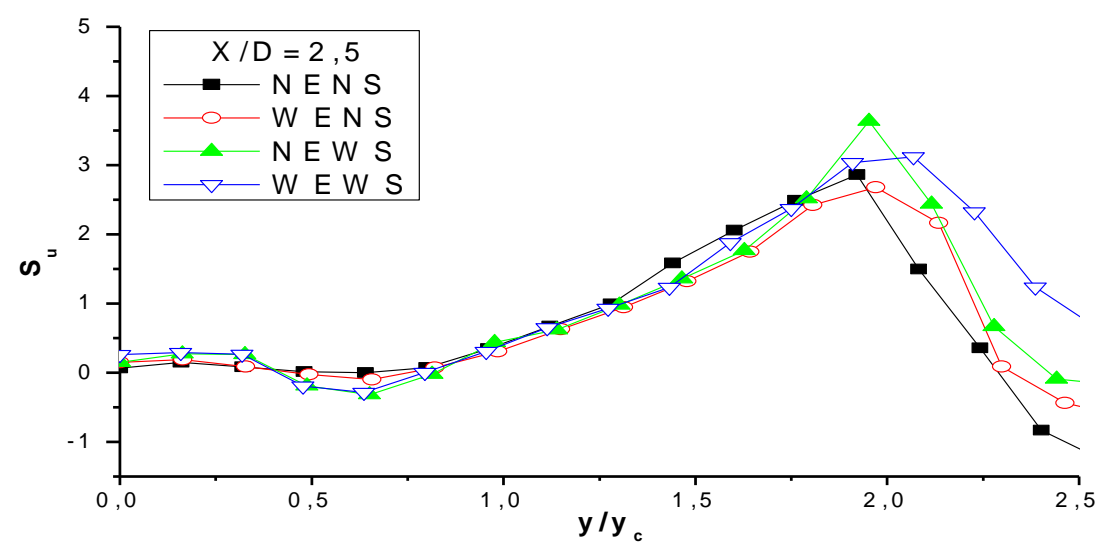

e)

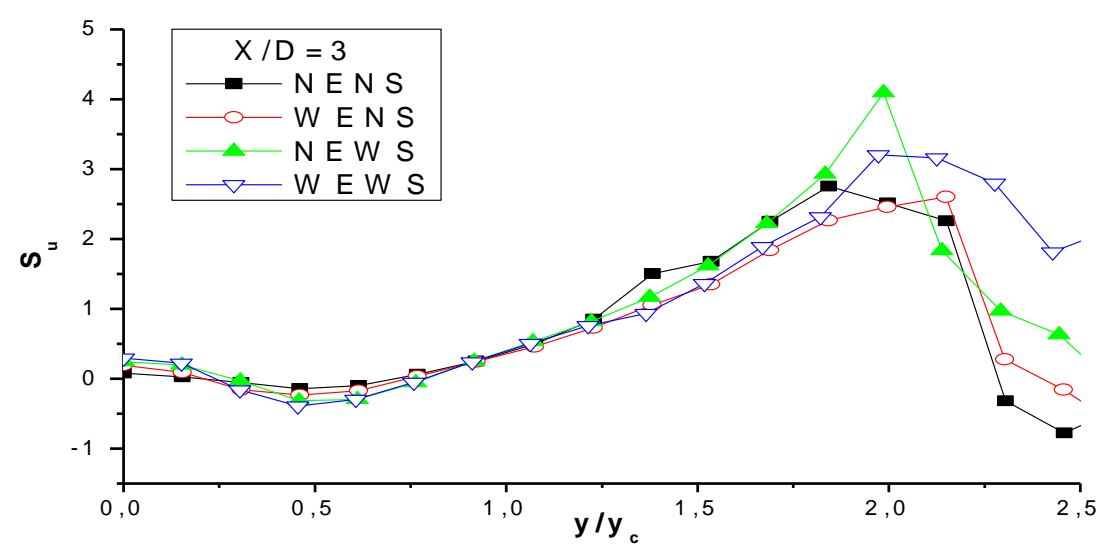

f)

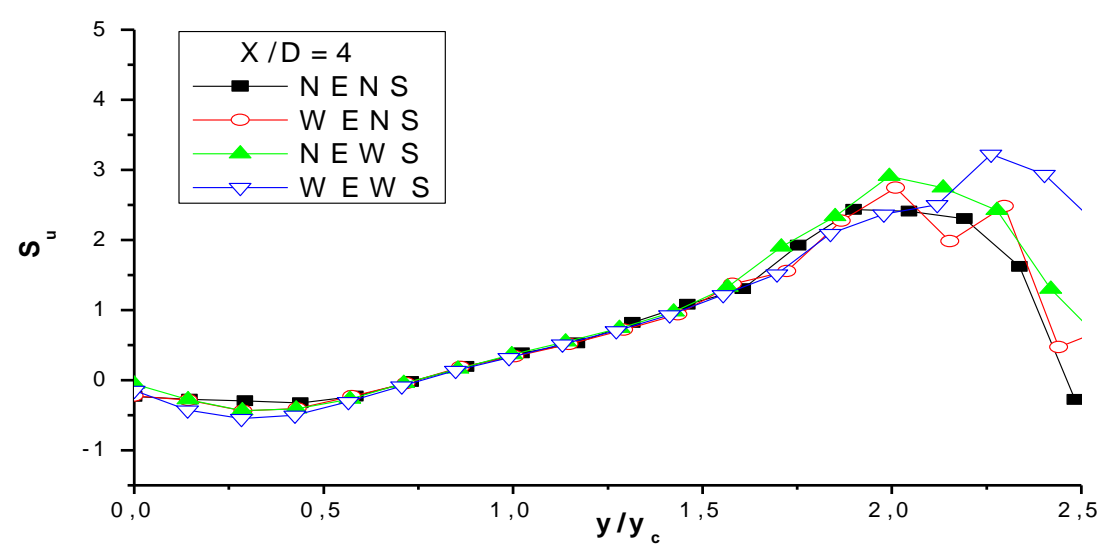

Figure 5.32: Skewness profiles of the longitudinal velocity on the central $x y$ plane at different downstream locations (cont'd). 
g)

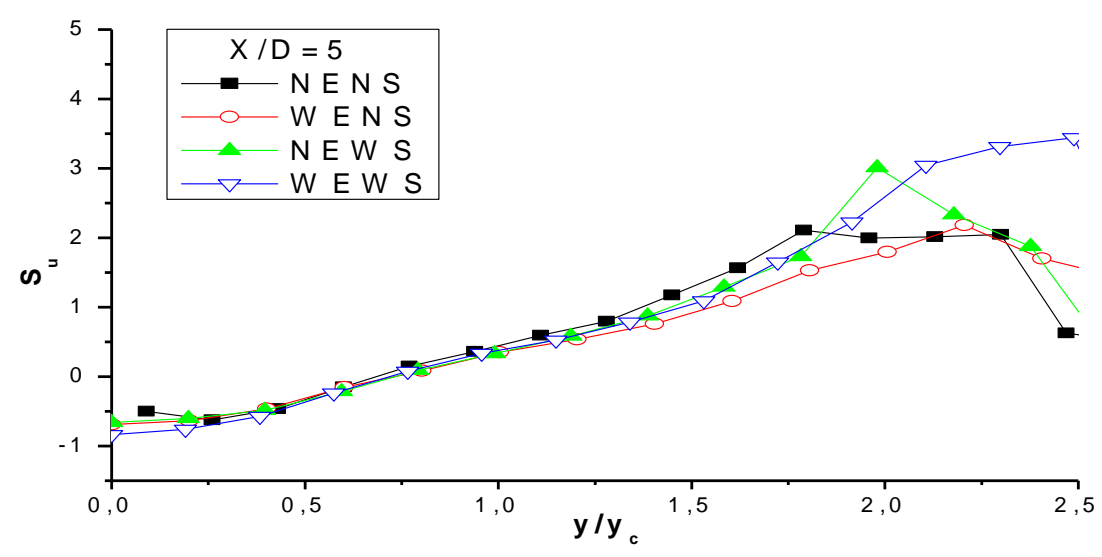

h)

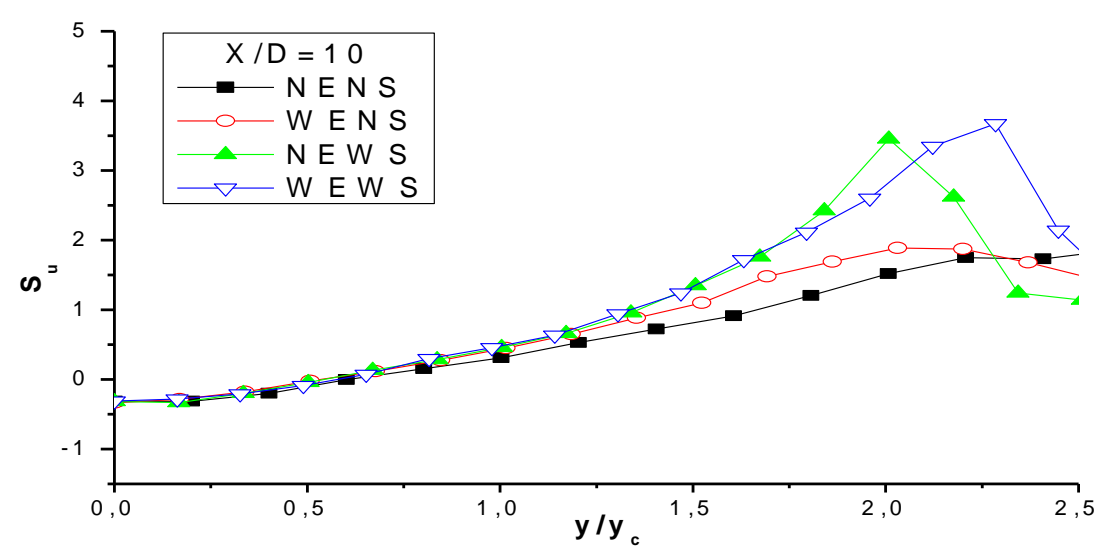

j)

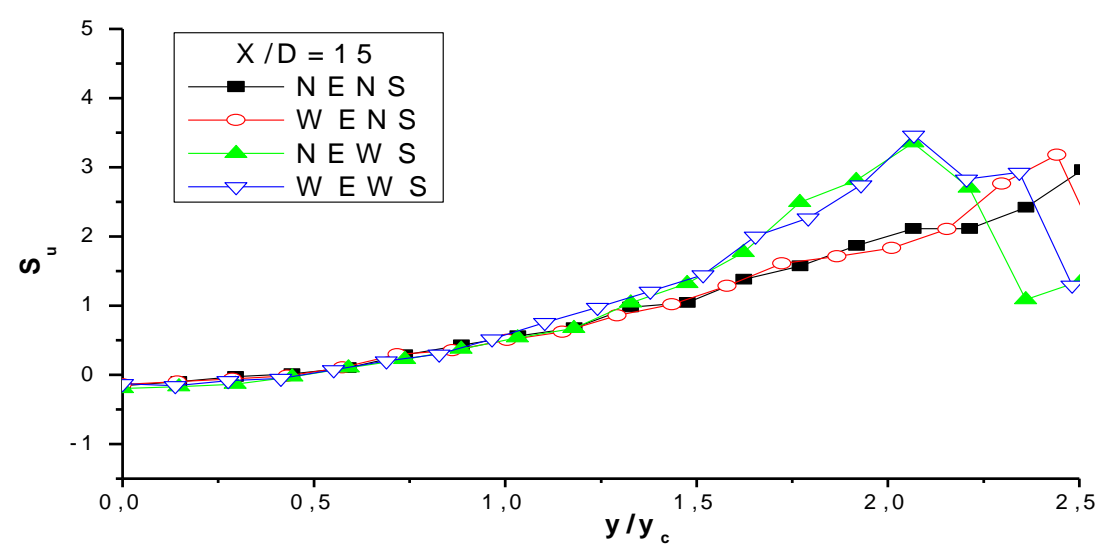

Figure 5.32: Skewness profiles of the longitudinal velocity on the central $x y$ plane at different downstream locations (cont'd). 
k)

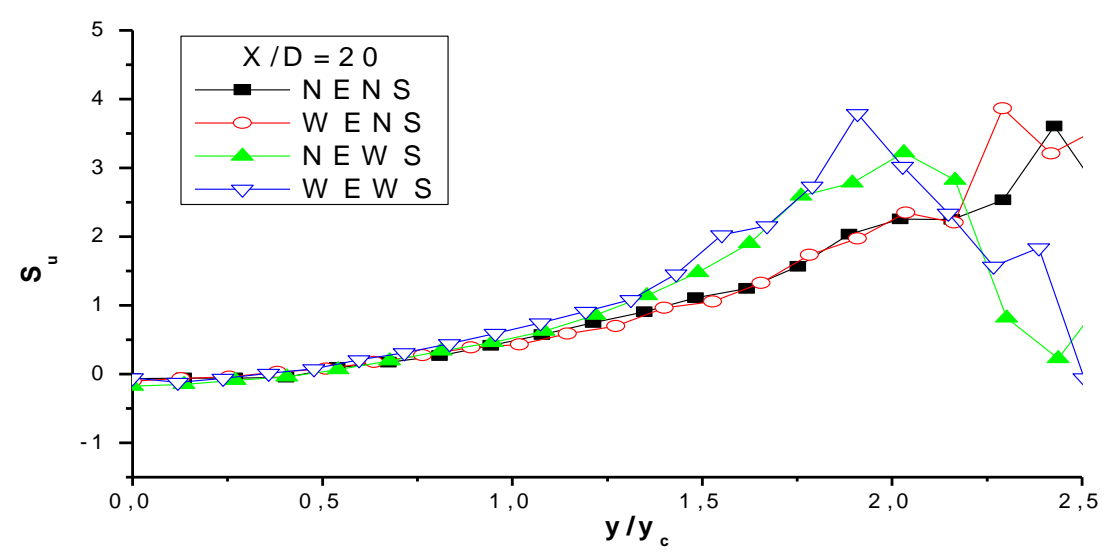

1)

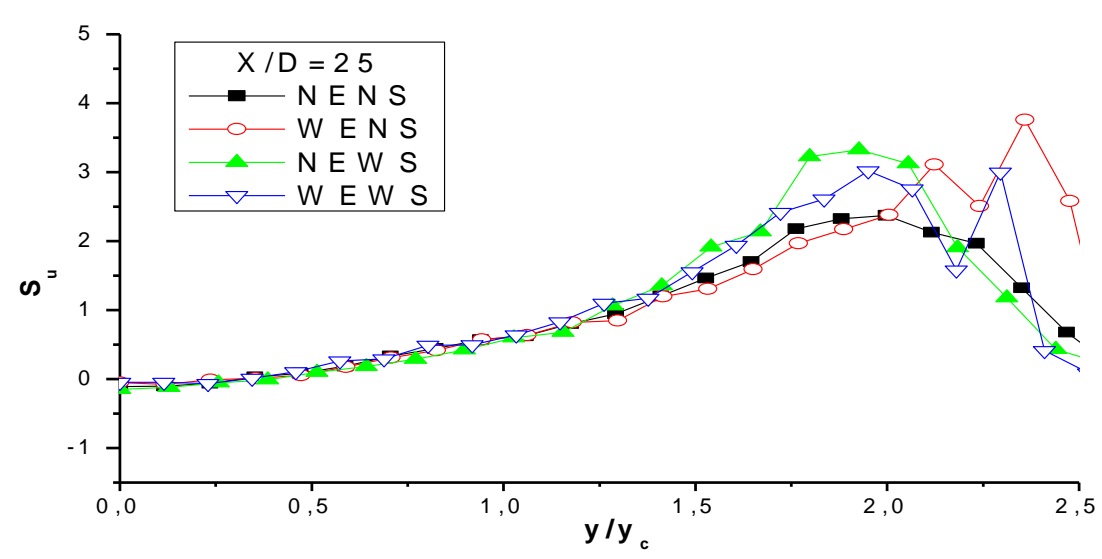

m)

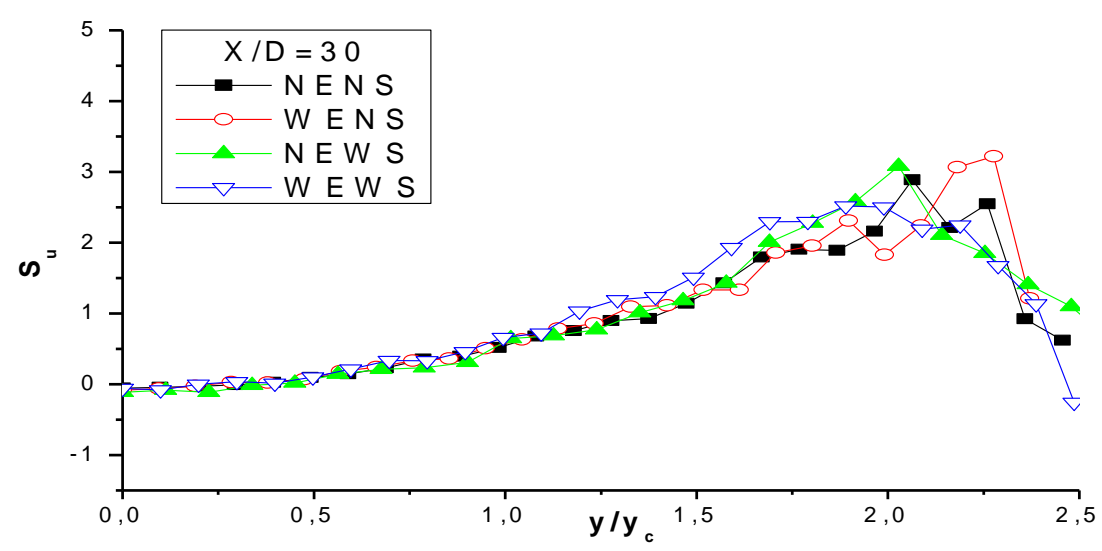

Figure 5.32: Skewness profiles of the longitudinal velocity on the central $x y$ plane at different downstream locations (cont'd). 
n)

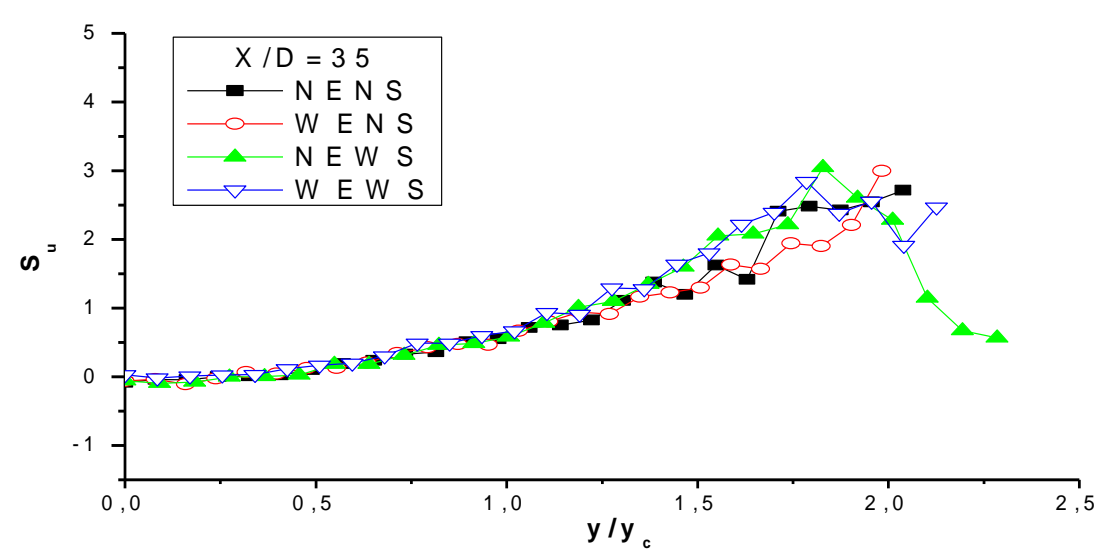

Figure 5.32: Skewness profiles of the longitudinal velocity on the central $x y$ plane at different downstream locations (cont'd).

a)

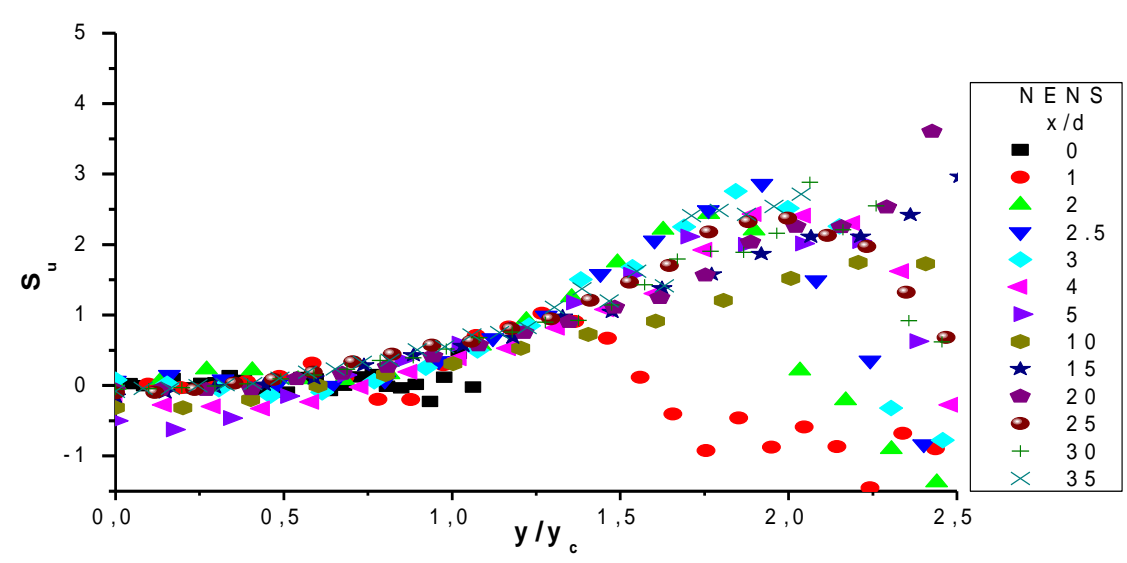

b)

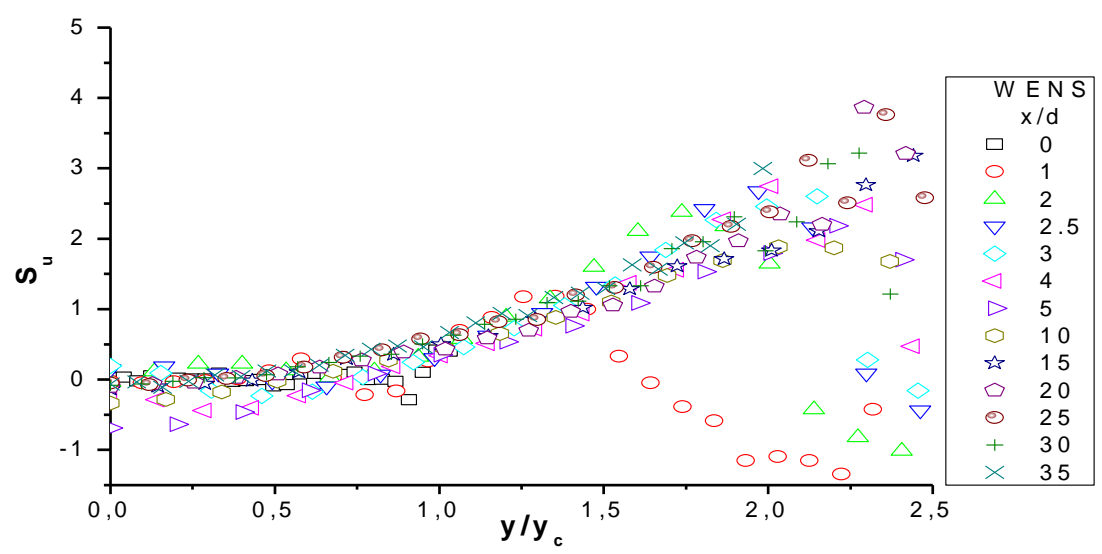

Figure 5.33: Self-similarities of skewness profiles of the longitudinal velocity on the central $x y$ plane for all test cases: a) NENS, b) WENS, c) NEWS and d) WEWS. 
c)

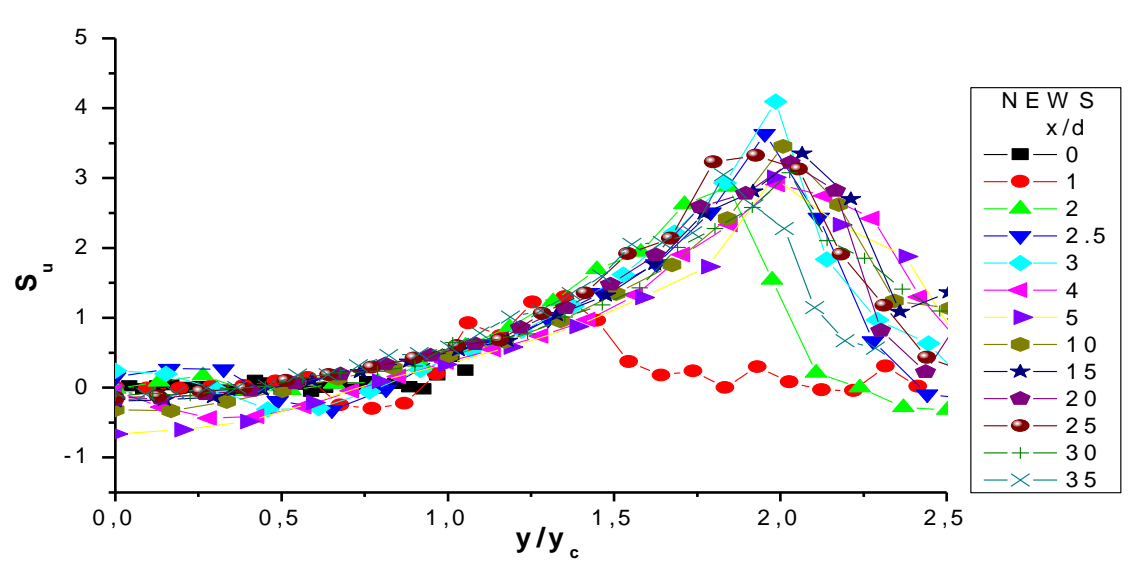

d)

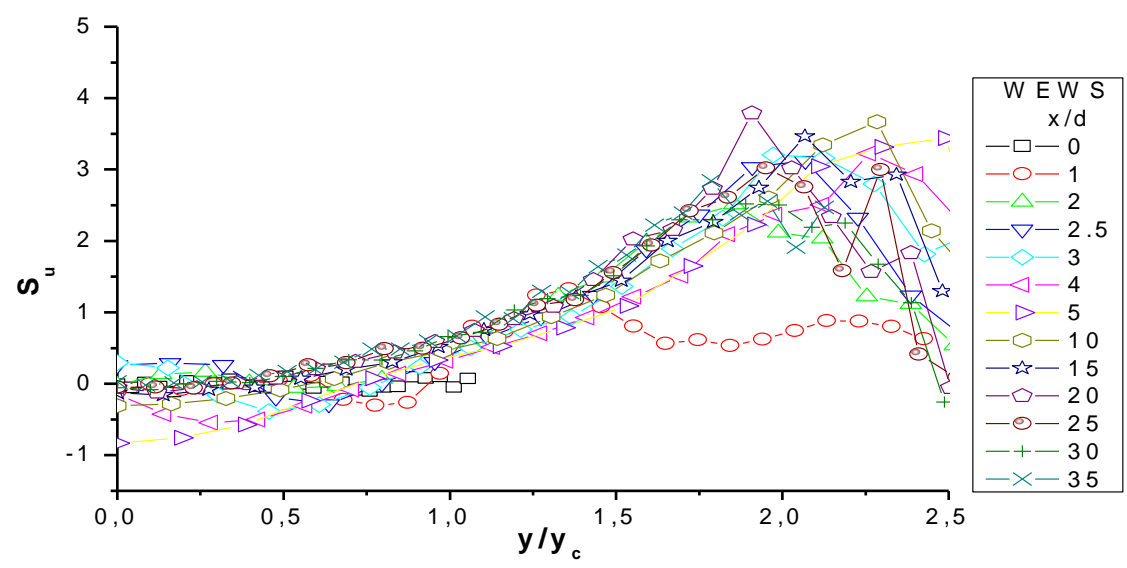

Figure 5.33: Self-similarities of skewness profiles of the longitudinal velocity on the central $x y$ plane for all test cases: a) NENS, b) WENS, c) NEWS and d) WEWS (cont'd).

b) Evolutions of the centreline skewness factor of the longitudinal velocity at various $R e$

Further insight into the flow field may be obtained from studying the centreline skewness factor of the streamwise velocity, $S_{u}$ at various speeds. The centreline distributions of the skewness factor in the axial direction up to $x / D=35$ are presented in Figures 5.34-a, b and c for $\operatorname{Re}=10,000,20,000$ and 30,000 respectively. It is demonstrated that the $S_{u}$ for all configurations varies dramatically in the near field. Further downstream, $S_{u}$ increases and then decreases, resulting in a local maximum and then local minimum before it reaches approximately to the saturation.

The local maximum and minimum evidenced in the present measurements have been observed in previews 2-d jets investigations (e.g. Deo et al., 2007a, Deo et al., 2008). 
The upper peak may be produced due to the growth and merging of large scale structures which are present at the end of the potential core region, whereas the lower peak is probably associated with the induction of low velocity ambient fluid, due to lateral oscillation of the jets' potential core (Deo, 2005). At $R e=10,000$, all configurations display a local maximum at $x / D=4$ and a local minimum at $x / D=9$. At $R e=20,000$, all configurations (NENS, WENS, NEWS and WEWS) display similar local maximum at $x / D=2.5$ whereas local minimum at $x / D=6$ and 5 for jets without sidewalls (NENS, WENS) and jets with sidewalls (NEWS and WEWS) respectively. At $R e=30,000$ all the jets produce upper peak at the same location i.e. $x / D=2.5$ but different location for the lower peak i.e. $x / D=6$ for NENS and WENS, $x / D=5$ for NEWS and WEWS. Although the location of positive peak for each configuration at all Reynolds number is found to be similar, the value of this peak is found to depend on the configuration. We have found that the positive peak in the jets with sidewalls (NEWS, WEWS) present lower values in comparison to jets without sidewalls (NENS, WENS). These trends are probably due to the more organized structures which produced in jets with sidewalls. This is compatible with the sharp peaks which were found at the edges of the jets with sidewalls (see the transverse distributions of $\mathrm{S}_{\mathrm{u}}$, Figure 5.32). Nevertheless, the location of the negative peak (or local minimum) is found to vary in the presence or the absence of the sidewalls. It is observed that the presence of the sidewalls (NEWS and WEWS) result in early establishment of these negative peaks, i.e. $x / D=7$, in comparison with free jets (NENS, WENS) i.e. $x / D=9$. These findings further confirm the effects that are imposed due to the presence or absence of the sidewalls and/or endplates.

a)

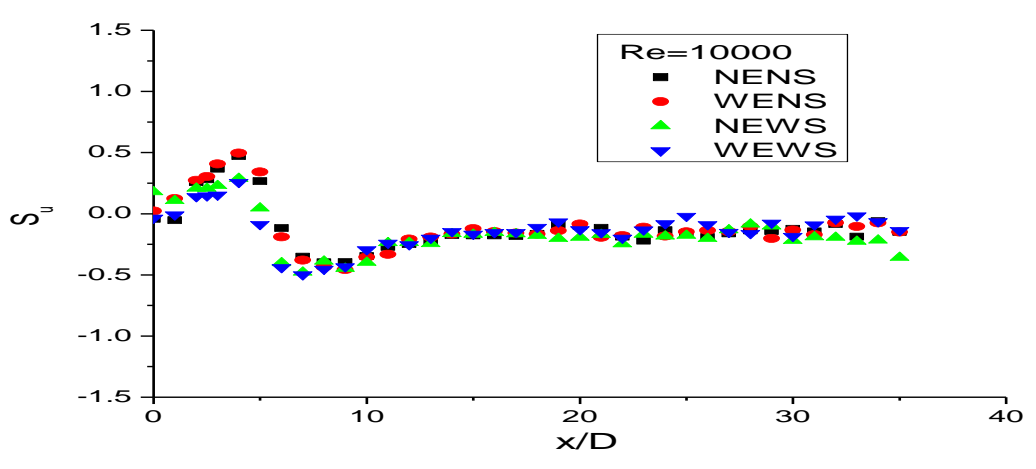

Figure 5.34: Centreline distributions of skewness factor of the longitudinal velocity for all configuration at various Reynolds numbers: a) 10,000, b) 20,000 and c) 30,000 
b)

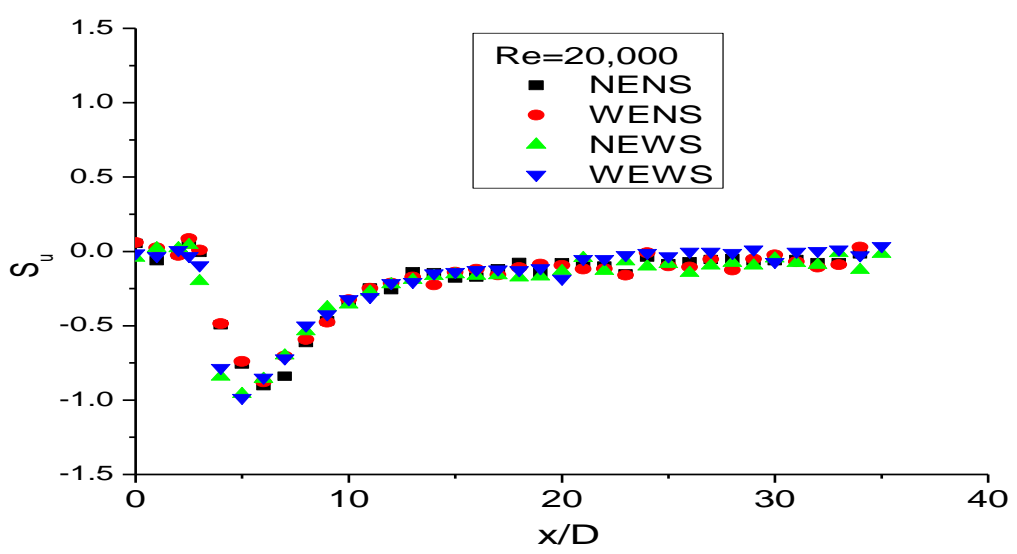

c)

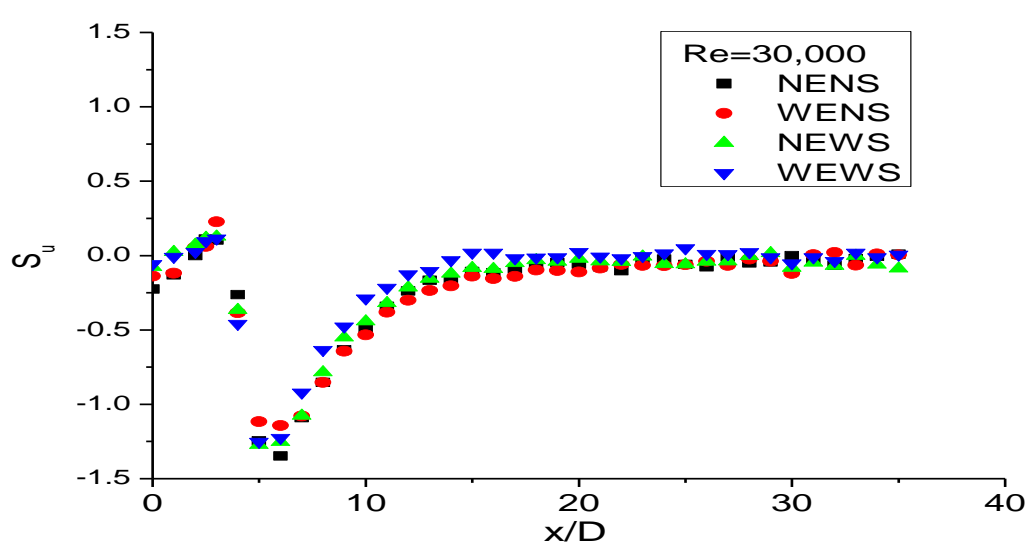

Figure 5.34: Centreline distributions of skewness factor of the longitudinal velocity for all configuration at Reynolds numbers: a) 10,000, b) 20,000 and c) 30,000 (con't)

It may be of interest to study the effect of high Reynolds number on the development of skewness factor e.g. $S_{u}$. Most of studies available in literature presenting the effect of Reynolds numbers on skewness factor, were confined to lower range of Reynolds number than ours (e.g. Deo et al., 2008, 1,500 $\geq R e \geq 16,500$ ). Therefore, the data of Figure 5.34 are rearranged based on configuration and are presented in Figure 5.35 to demonstrate any possible effects of the present range $(10,000 \geq R e \geq 30,000)$. As one can see, the effect of high Reynolds number on $S_{u}$ still exists. For all configurations, at the initial stages, three different distributions corresponding to $R e=10,000,20,000$ and 30,000 are observed. The location and the value of the local maximum are found to be related to Reynolds number. When $R e$ increases, the distance where the positive peak is displayed increases in the axial direction whereas its value decreases. These findings are compatible with published results on lower range $R e$ (e.g Deo et al., 2008). But in the presence of the sidewalls are found to suppress these peaks, as 
discussed earlier (see Figure 5.34). Similarly, as for the positive peak (i.e. local maximum), the location and value of the negative peak (i.e. local minimum) is found to depend on Reynolds numbers. As shown in Figure 5.35, for all configurations, with the increase of Reynolds number the value of the local minimum also increases. Beyond $x / D=10$, the values of $S_{\mathrm{u}}$ vary towards their asymptotic value.

a)

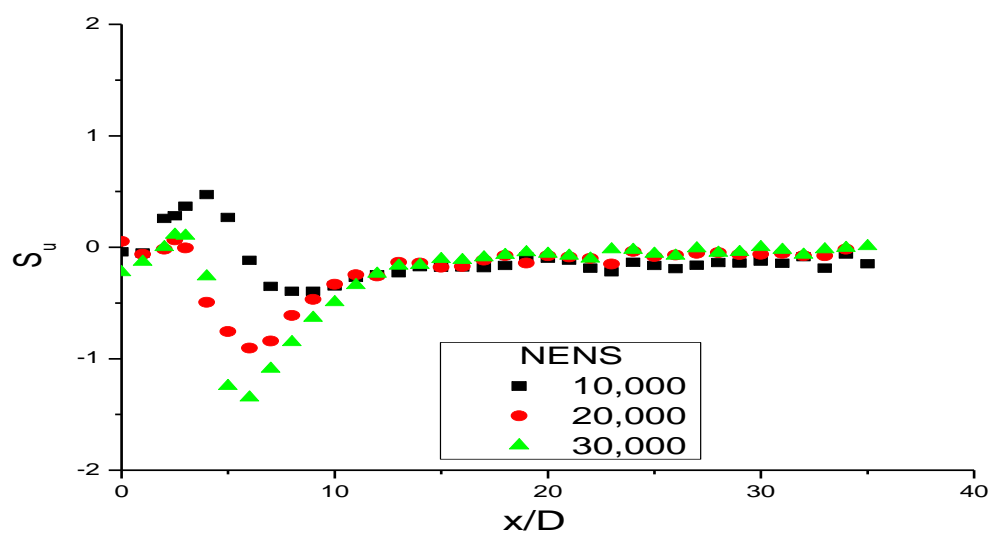

b)

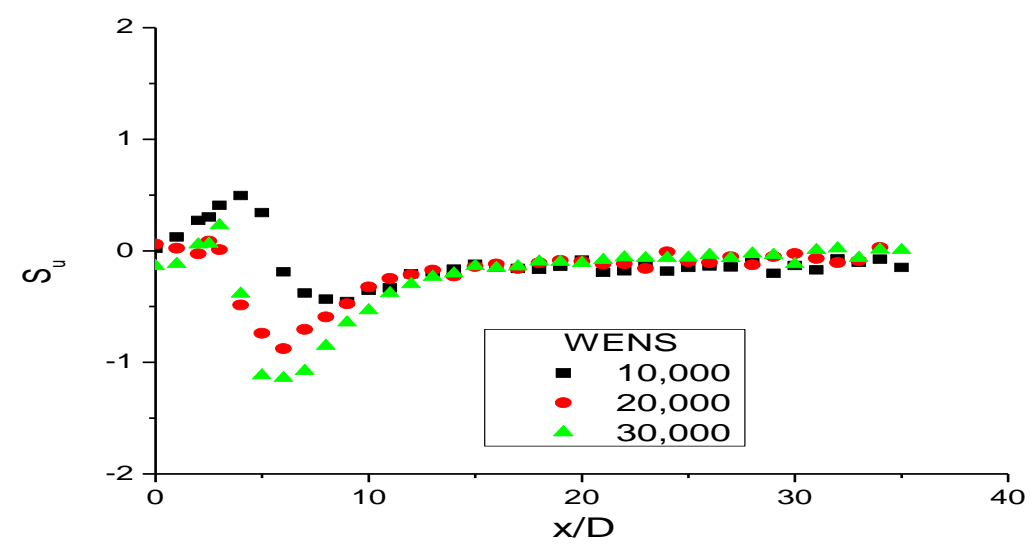

Figure 5.35: Reynolds number, $R e$ dependence of the centerline skewness factor of the longitudinal velocity for: a) NENS, b) WENS, c) NEWS and d) WEWS. 
c)

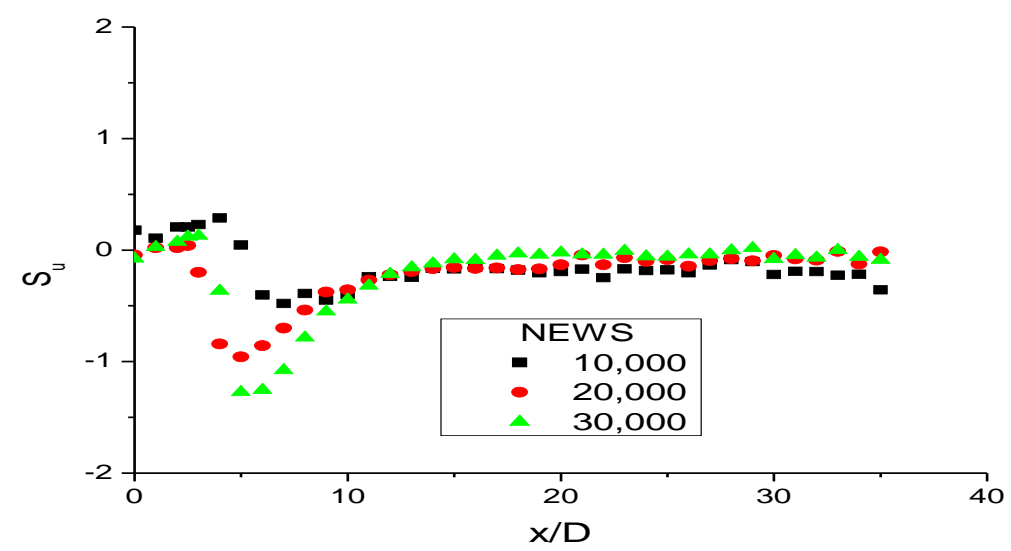

d)

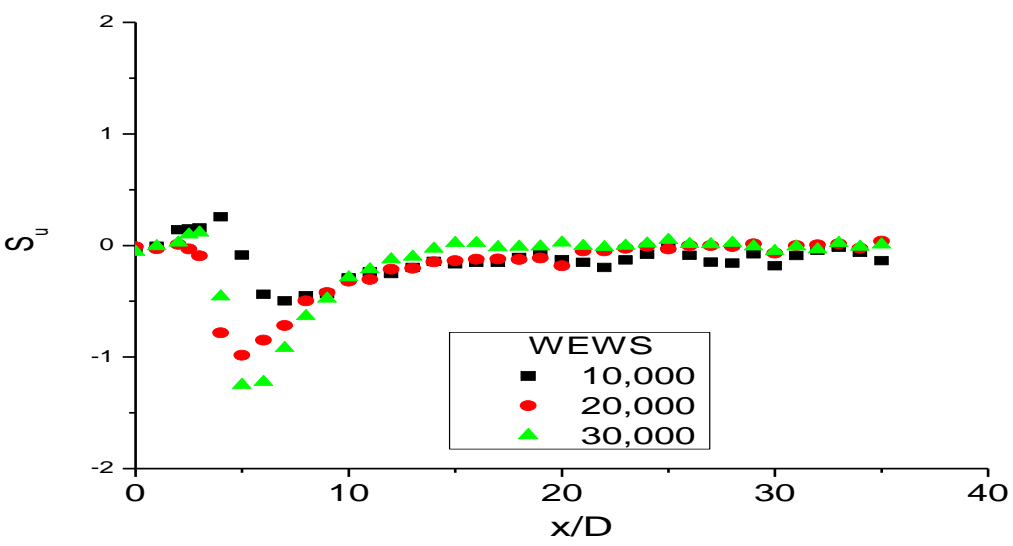

Figure 5.35: Reynolds number, $R e$ dependence of the centerline skewness factor of the longitudinal velocity for: a) NENS, b) WENS, c) NEWS and d) WEWS (con't). 


\section{c) Transverse distributions of skewness factor of the lateral velocity}

The transverse distributions of the skewness factor of the lateral velocity, $S_{v}=\left\langle v^{3}\right\rangle$ | $v^{\prime 3}$ are shown in Figures 5.36-a, b, c, d, e, f, g, h, j, k, l, m and $\mathrm{n}$ for downstream locations $x / D=0,1,2,2.5,3,4,5,10,15,20,25,30$ and 35 respectively. In these graphs the differences are again minimised and in most cases the curves can be distinguished on the basis of the presence or absence of sidewalls where remarked that the most characteristic attributes of skewness distributions, distinguishing the configurations comprising sidewalls are the sharp peaks developing towards the outer edges.

a)

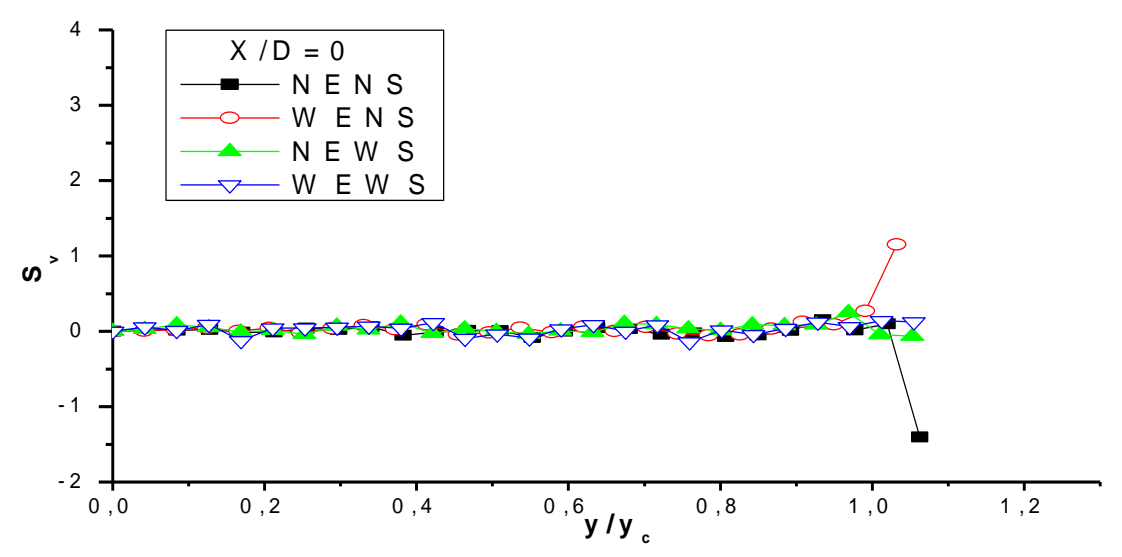

b)

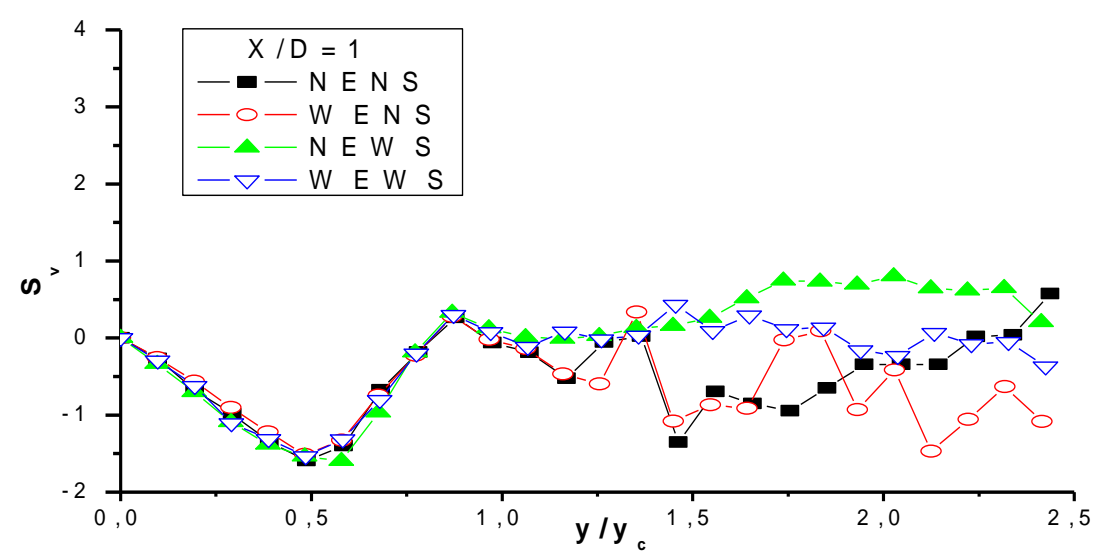

Figure 5.36: Skewness profiles of the lateral velocity on the central $x y$ plane at different downstream locations. 
c)

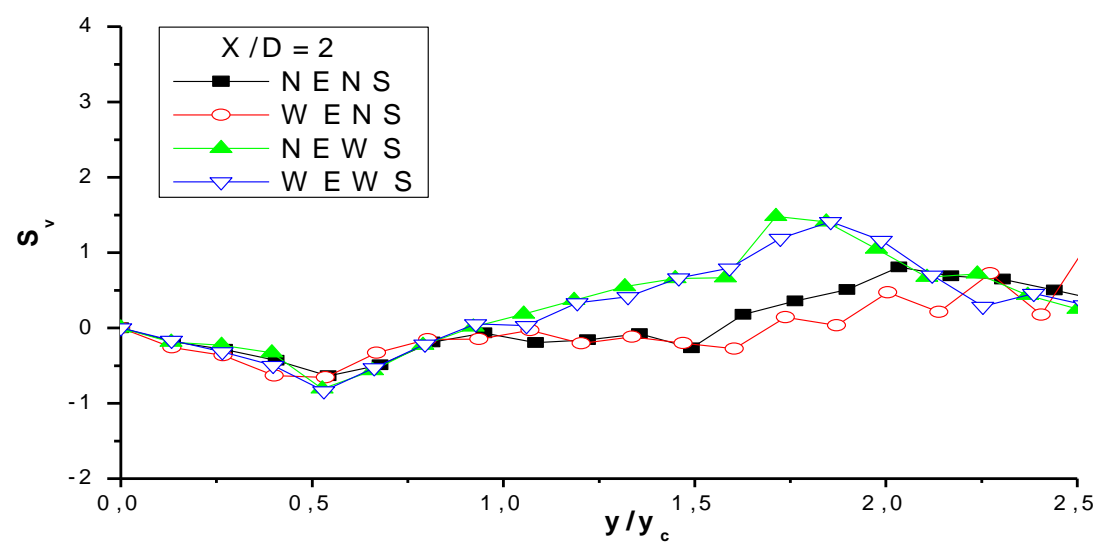

d)

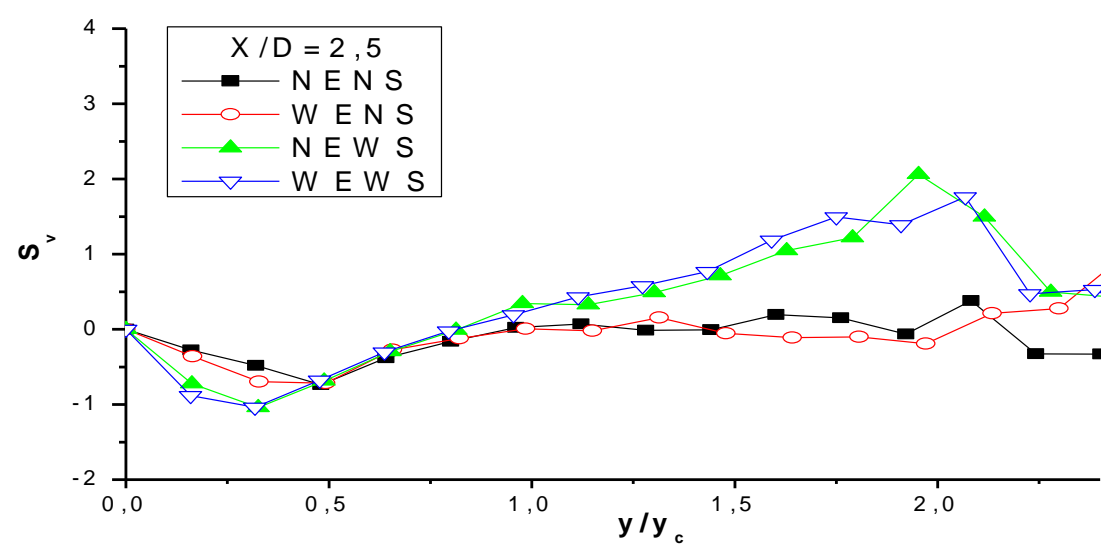

e)

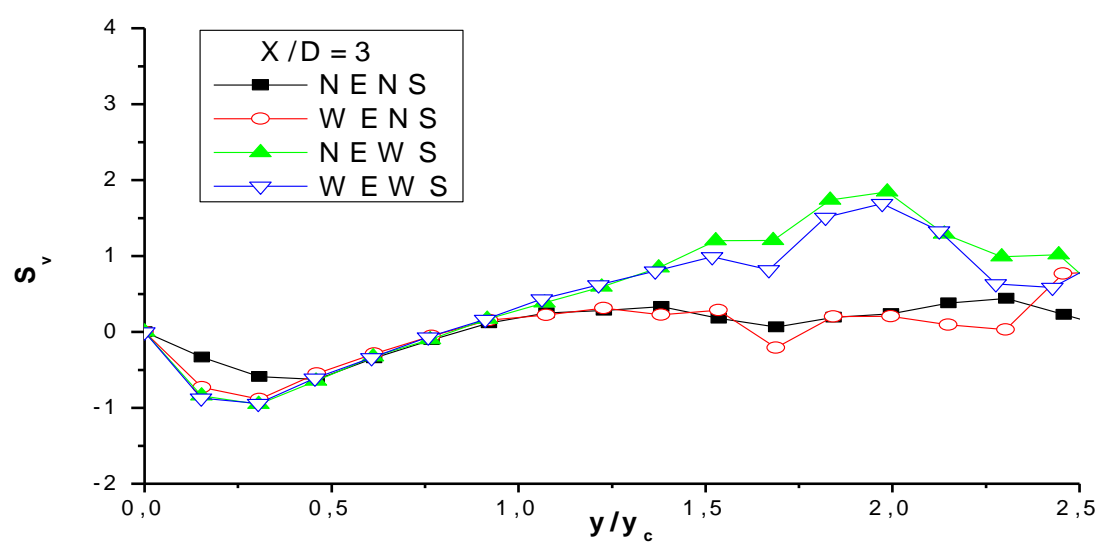

Figure 5. 36: Skewness profiles of the lateral velocity on the central $x y$ plane at different downstream locations (con't). 
f)

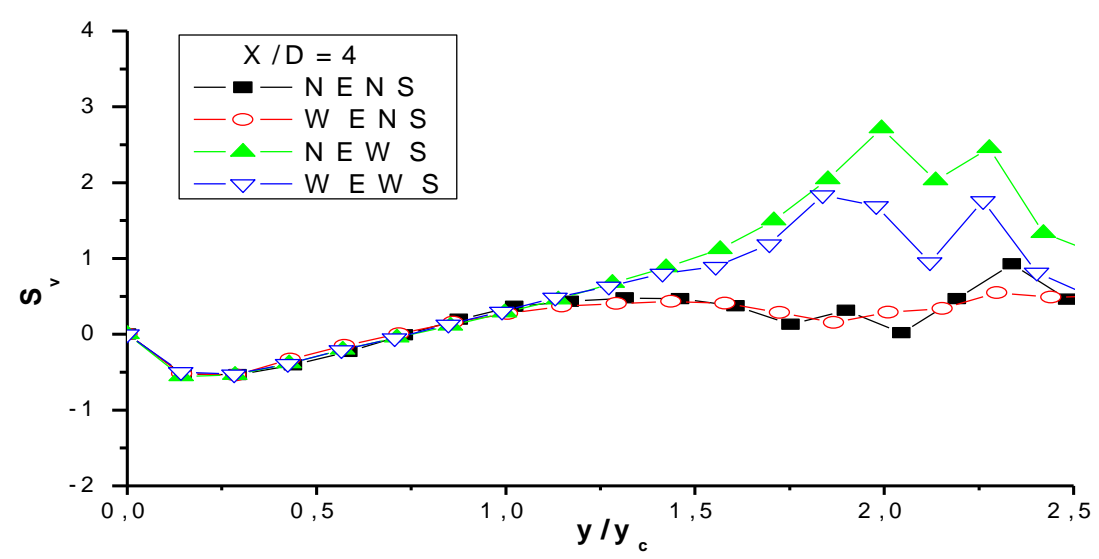

g)

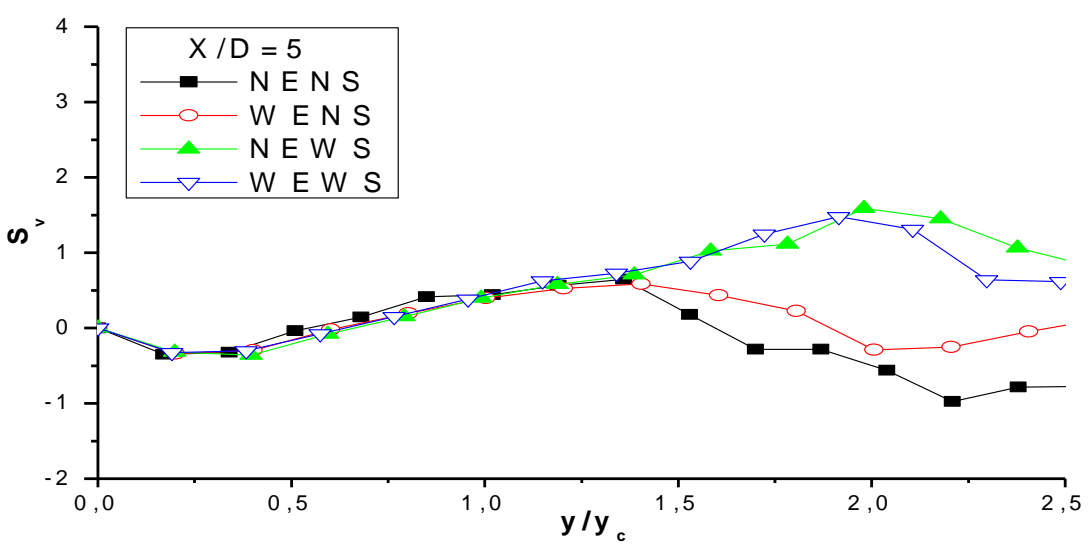

h)

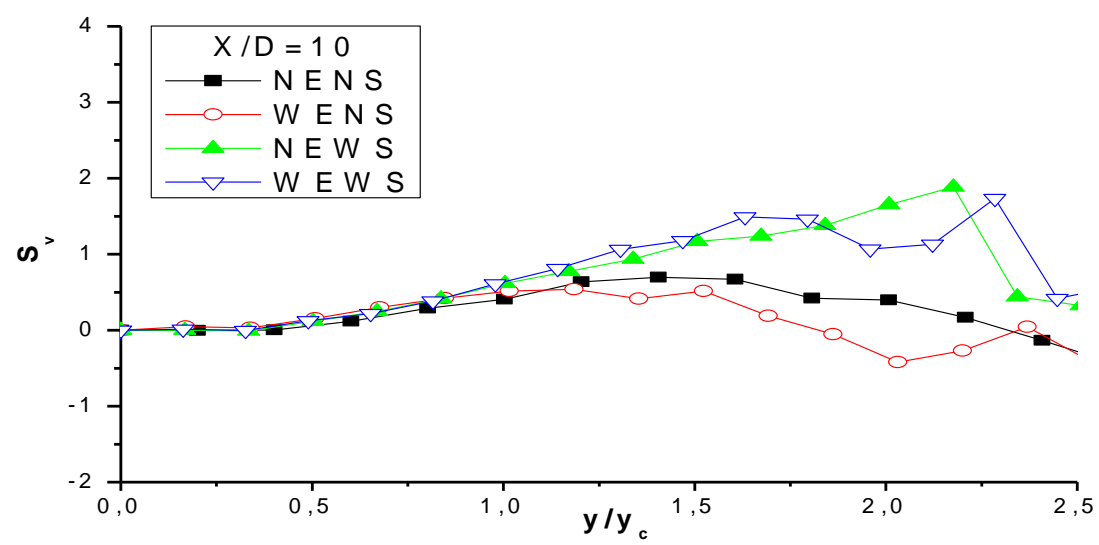

Figure 5. 36: Skewness profiles of the lateral velocity on the central $x y$ plane at different downstream locations (con't). 
j)

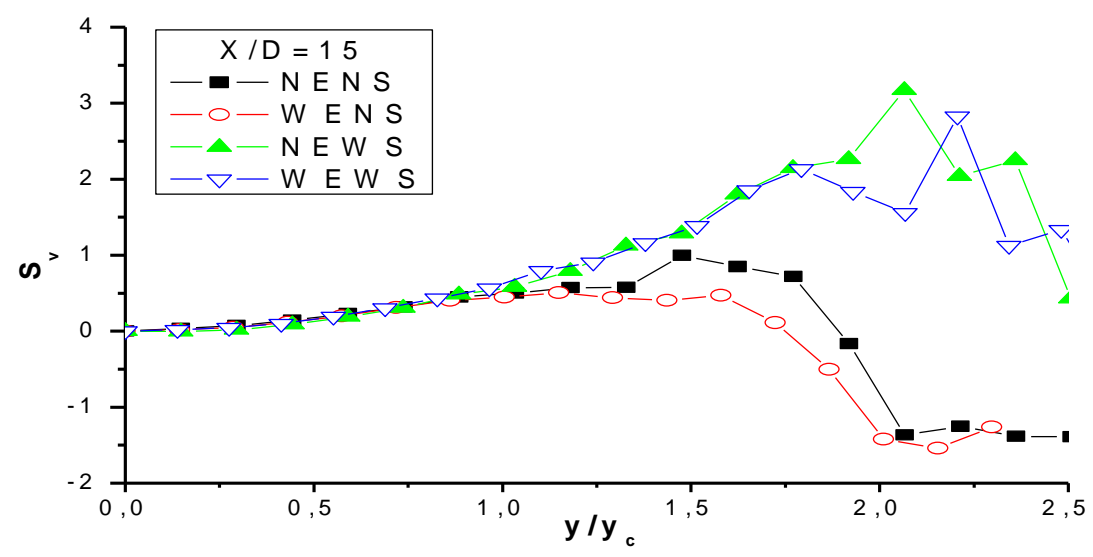

$\mathrm{k})$

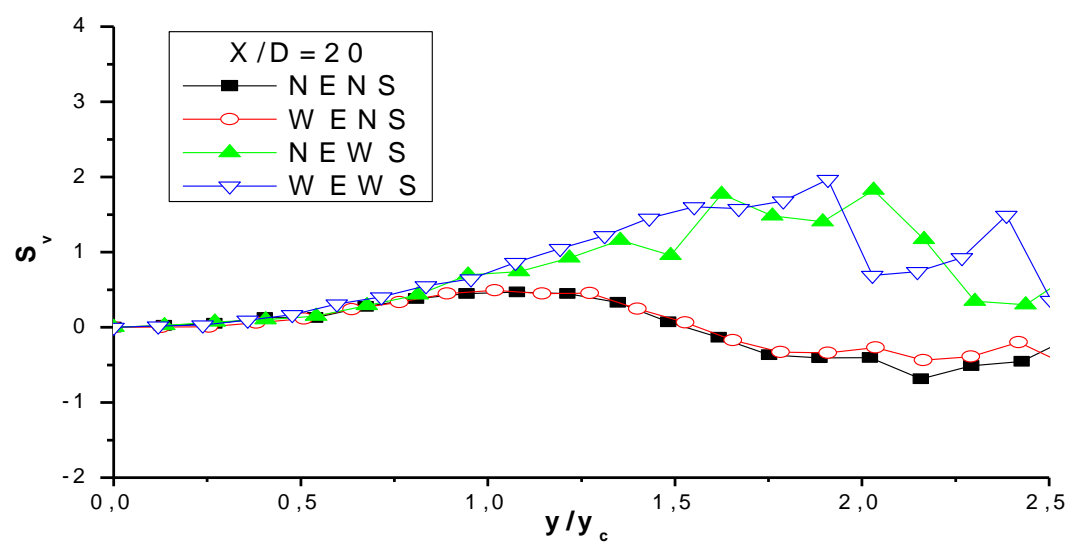

1)

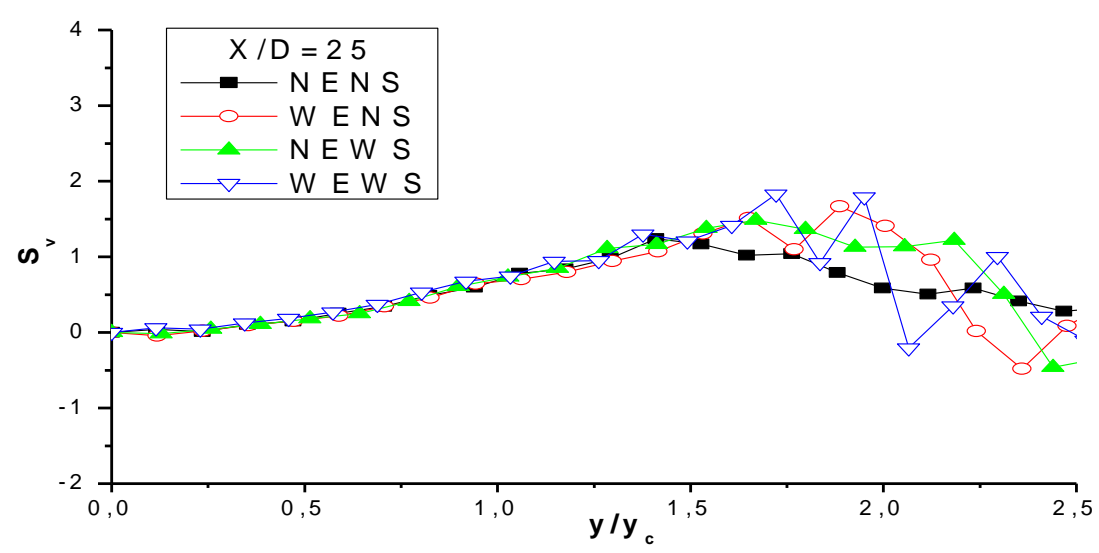

Figure 5. 36: Skewness profiles of the lateral velocity on the central $x y$ plane at different downstream locations (con't). 
$\mathrm{m})$

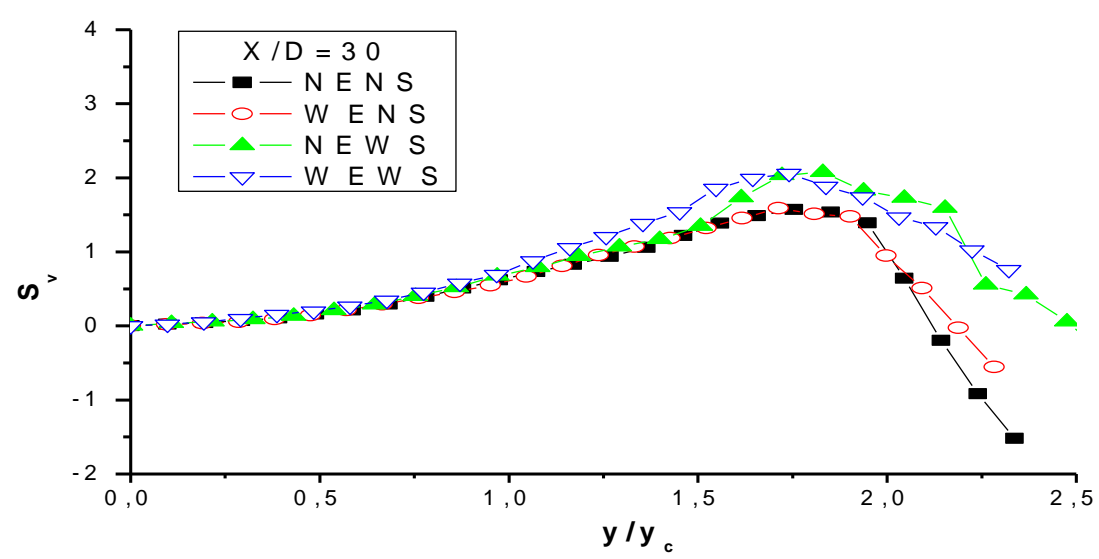

n)

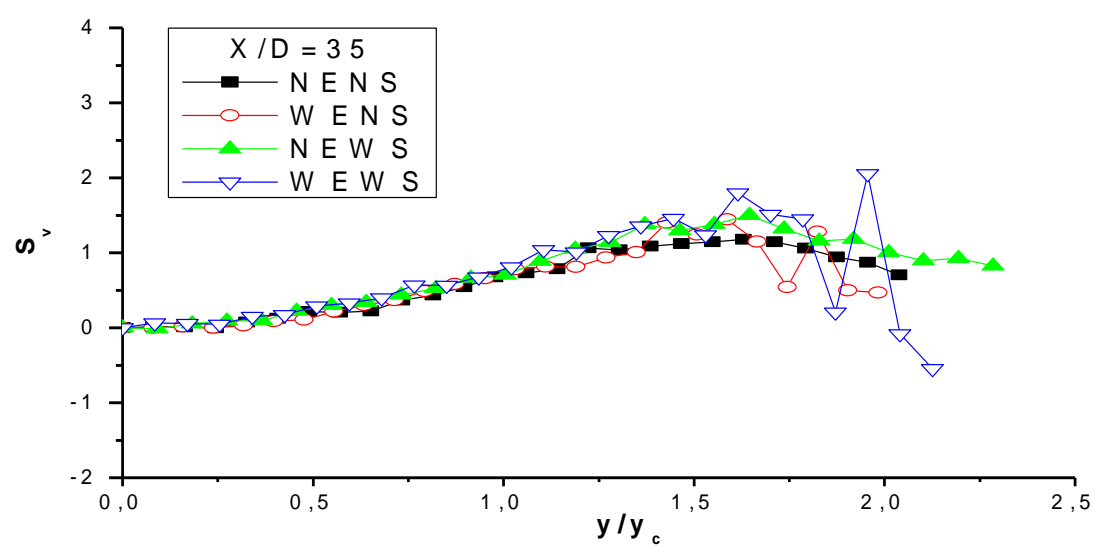

Figure 5.36: Skewness profiles of the lateral velocity on the central $x y$ plane at different downstream locations (cont'd).

a)

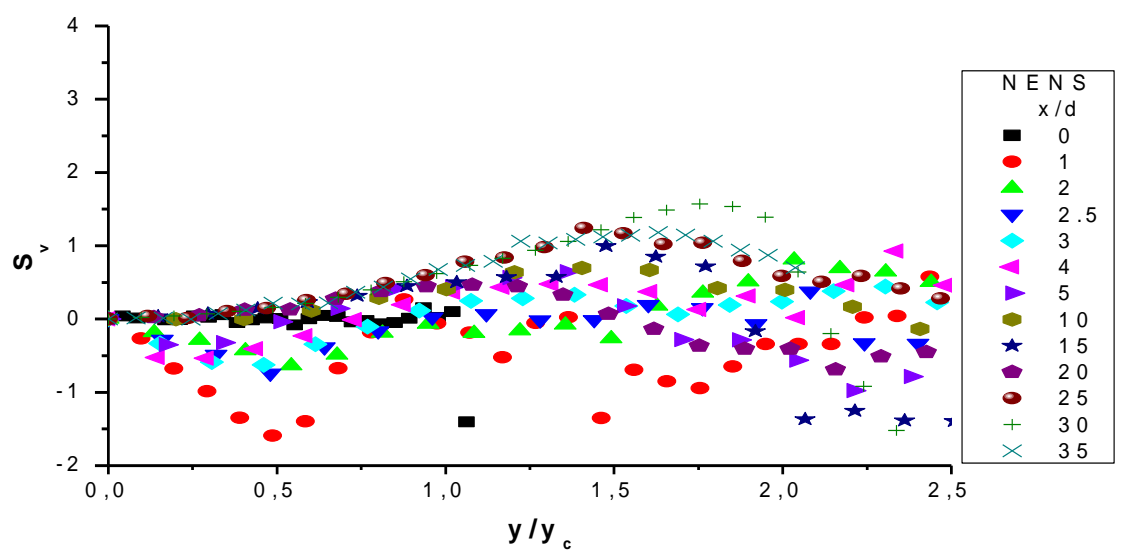

Figure 5.37: Self-similarities of skewness profiles of the lateral velocity on the central $x y$ plane for all test cases: a) NENS, b) WENS, c) NEWS and d) WEWS. 
b)

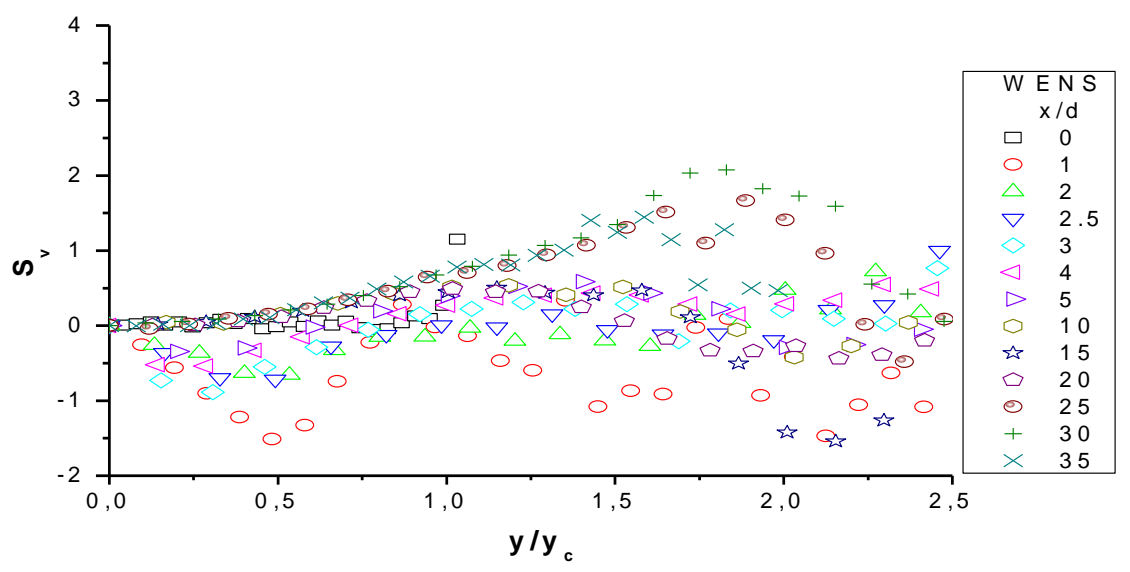

c)

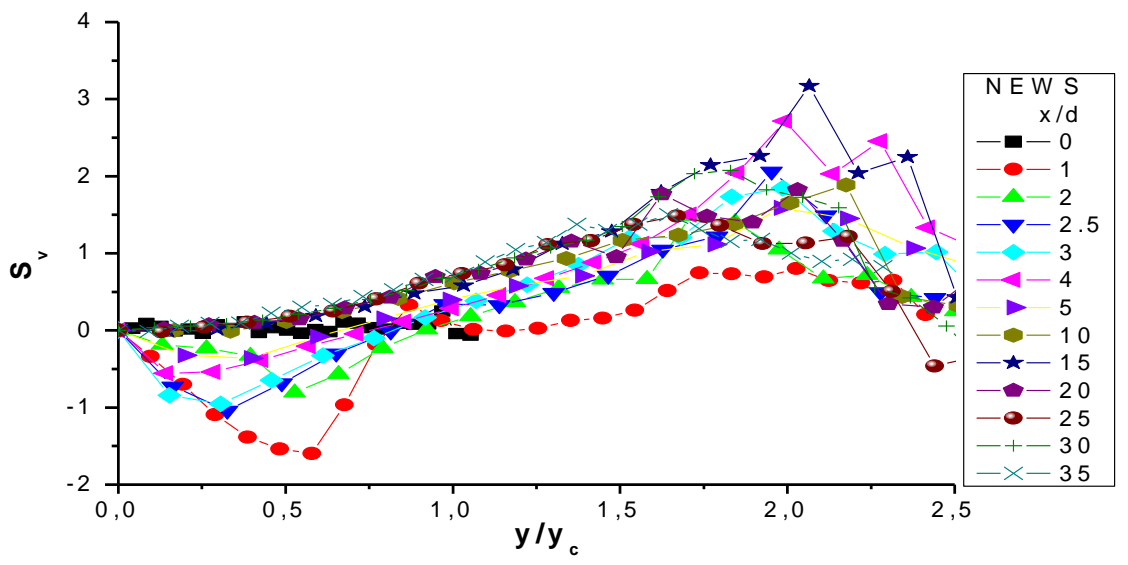

d)

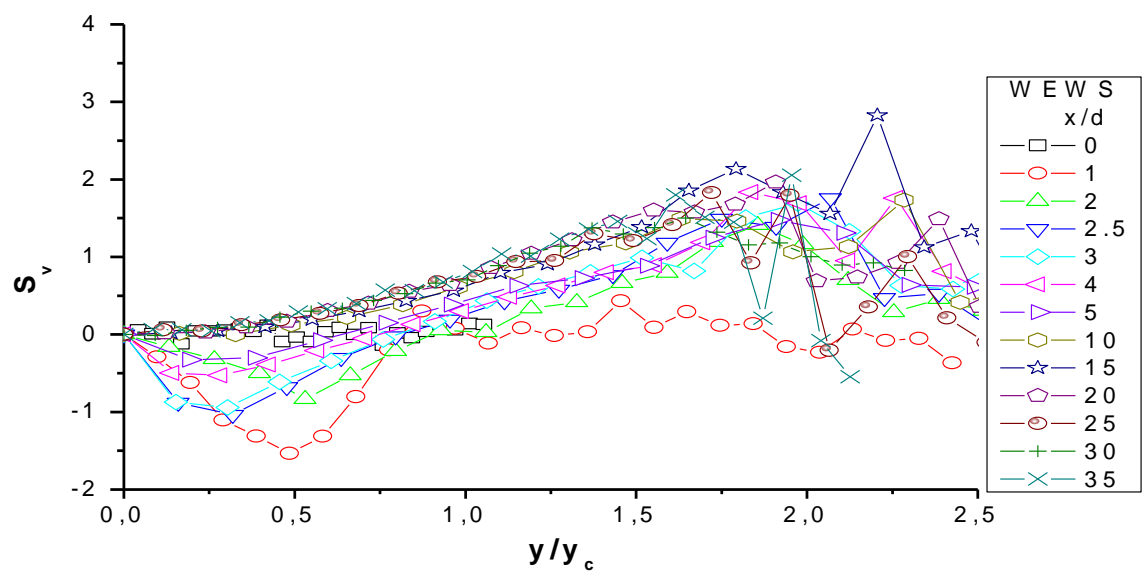

Figure 5.37: Self-similarities of skewness profiles of the lateral velocity on the central $x y$ plane for all test cases: a) NENS, b) WENS, c) NEWS and d) WEWS (cont'd). 
d) Evolutions of the centreline skewness factor of the lateral velocity at various $\mathrm{Re}$

The evolutions of skewness factor of the lateral velocity, $S_{v}$ for all configurations are presented in Figures 5.38-a, b and $\mathrm{c}$ for $R e=10,000,20,000$ and 30,000. Measurements were collected at the jet's centreline up to downstream location of 35 nozzle width. The shapes of the distributions of $S_{v}$ for the four configurations are found to be different, distinguishing jets without sidewalls (NENS, WENS) from jets with sidewalls (NEWS, WEWS) which also vary by changing Reynolds number.

a)

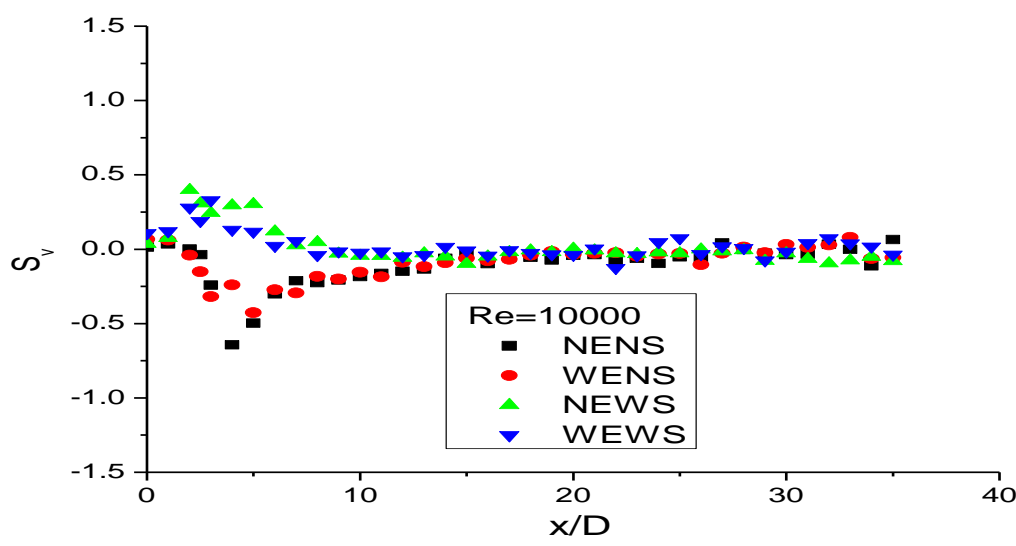

b)

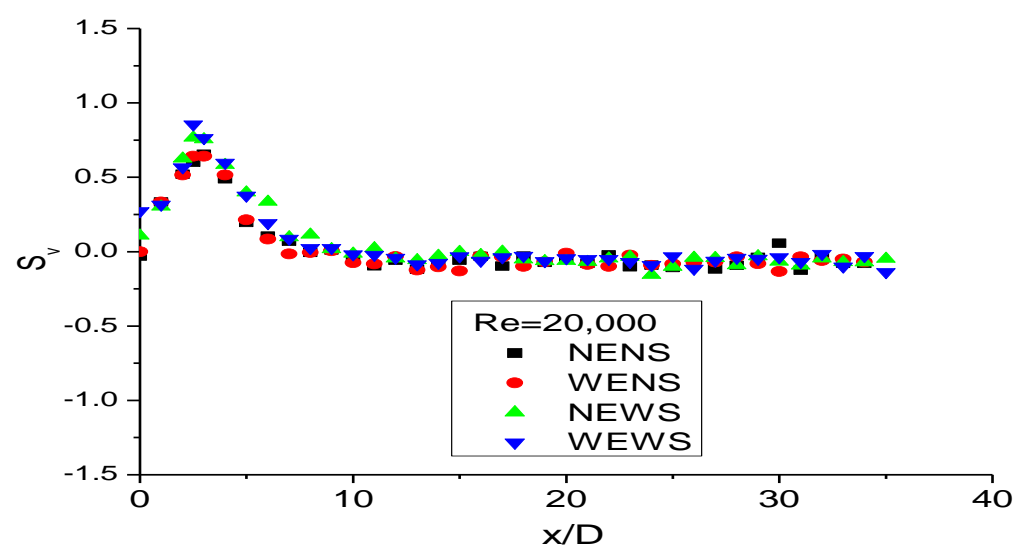

Figures 5.38: Centreline distributions of skewness factor of the lateral velocity for all configurations at Reynolds numbers: a) 10,000, b) 20,000 and c) 30,000 . 
c)

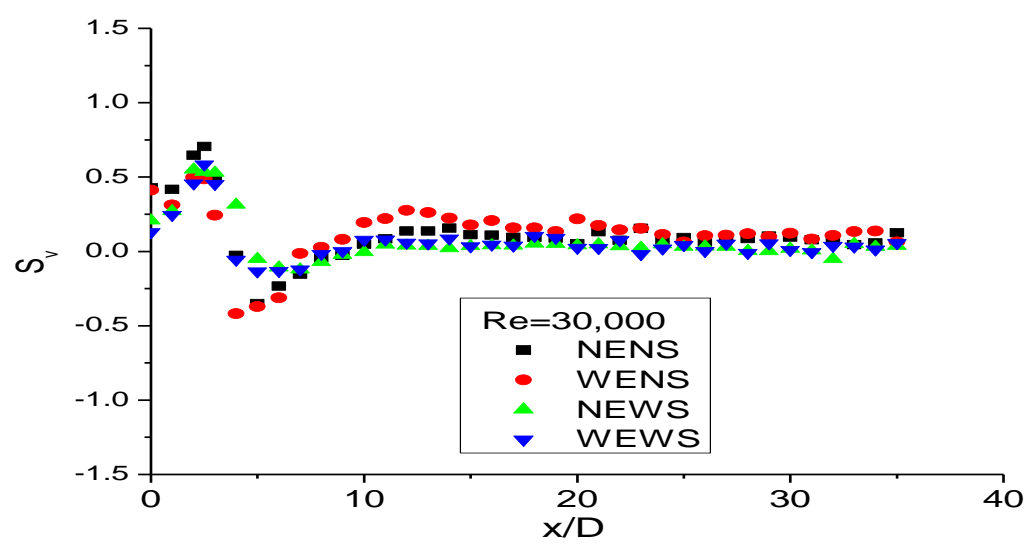

Figures 5.38: Centreline distributions of skewness factor of the lateral velocity for all configuration at Reynolds numbers: a) 10,000, b) 20,000 and c) 30,000 (con't)

Figure 5.39 demonstrates the effect of Reynolds number on $S_{v}$ for all the configurations. Surprisingly, the trend of $S_{v}$ for each configuration varies with Reynolds number. For NENS and WENS, at $R e=10,000, S_{v}$ doesn't display local maximum whereas at $R e=20,000$ and 30,000, a local maximum is observed at $x / D=2.5$. While the local minimum is observed only at $R e=10,000$ and 30,000. Beyond $x / D=10, S_{v}$ varies dramatically with higher values at the highest Reynolds number. For jets with sidewalls (NEWS, WEWS), at all Reynolds numbers, a local maximum is observed. While local minimum was only observed at the highest $R e$. Beyond $x / D=10, S_{v}$ varies closely to the Gaussian value of zero, compared to the free jets (NENS, WENS) which deviate from the Gaussian. This is probably due to the improved two-dimensionality of the flow in the presence of the sidewalls. The lack of information regarding the evolution of $S_{v}$ in the literature makes any further assessment on its development impossible. 
a)

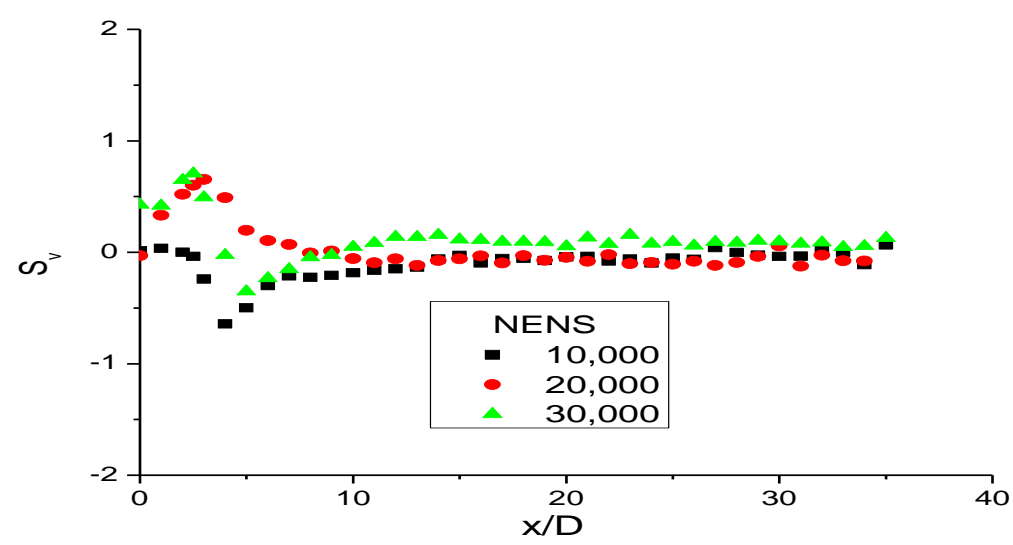

b)

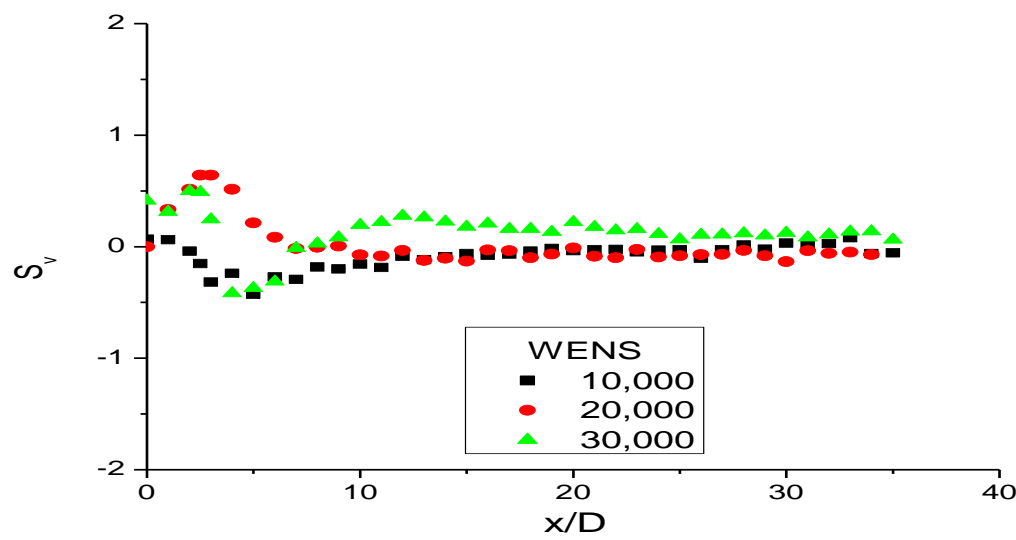

c)

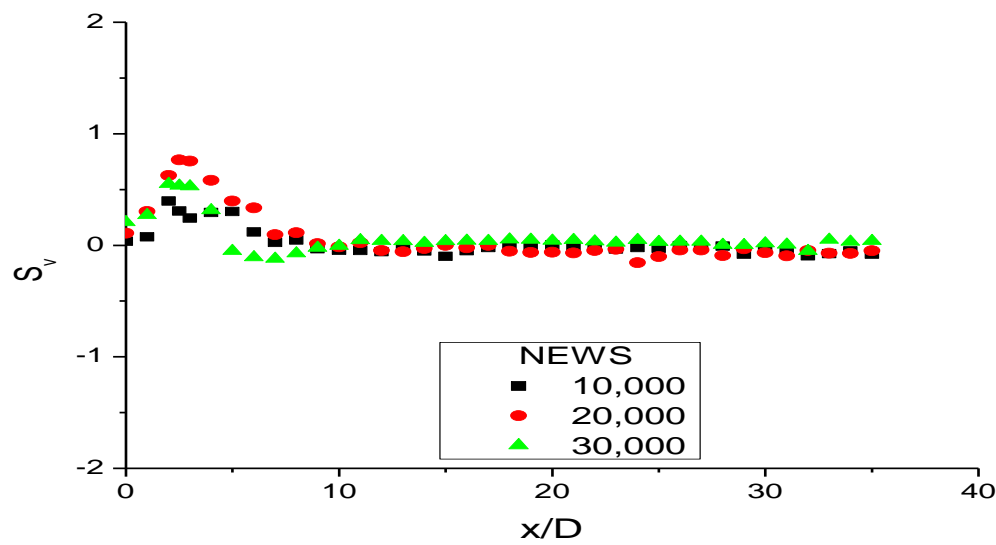

Figure 5.39: Reynolds number, $R e$ dependence of the centerline skewness factor of the lateral velocity for: a) NENS, b) WENS, c) NEWS and d) WEWS. 
d)

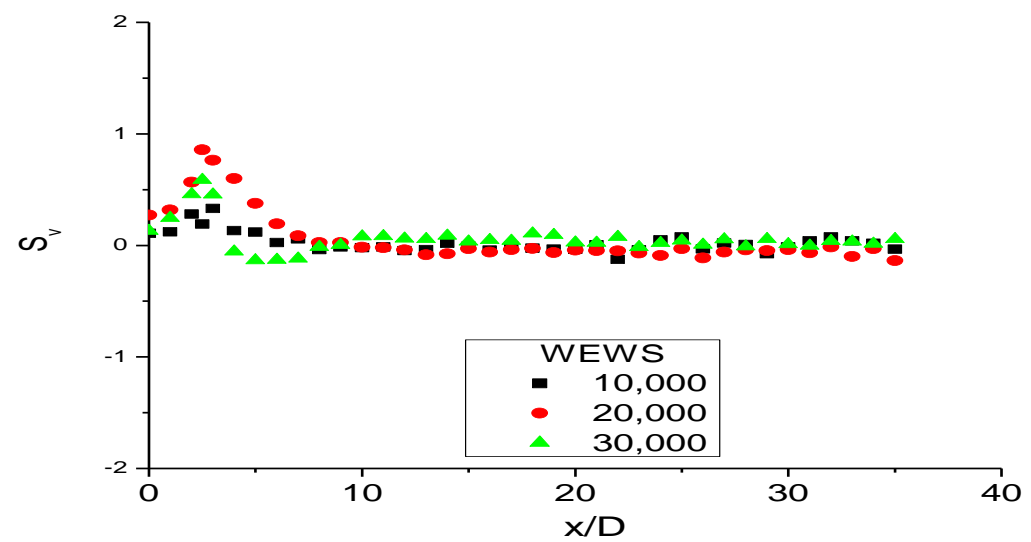

Figure 5.39: Reynolds number, $R e$ dependence of the centerline skewness factor of the lateral velocity for: a) NENS, b) WENS, c) NEWS and d) WEWS (con't). 


\subsection{Overview and physical interpretation of the experimental results}

Sidewalls (with or without endplate) have been used in many previous investigations (e.g. Bradbury, 1965, Gutmark \& Wygnanski, 1976, Browne et al., 1982, Everitt \& Robins, 1978, Browne et al., 1984, Deo et al., 2007a, Suresh et al., 2008), in most of the cases as a plausible means to increase the two dimensionality of the jet and generate a flow field resembling that of an infinite plane jet. The present results indicate that, the latter objective is only partly satisfied. The sidewalls indeed seem to increase the two-dimensionality, but establish distinct boundary conditions due to the shear on the wall and also impose a length scale equal to the length of the exit slot.

The entrainment mechanism dominating jet evolution is initiated by a KelvinHelmholtz instability of the shear layer between the jet and the ambient fluid which leads to the formation of vortices along the jet-ambient interface (Grinstein et al., (1995)). These vortices range from roller type linear vortices to ring vortices for the limiting cases of a 2D (Browne et al., 1982) or a round jet (Becker \& Massaro, 1968, Crow \& Champagne, 1971) whereas skewed ring vortices have been observed in rectangular jets of finite aspect ratio (Grinstein, 2001). At further stages the entrainment is dictated by the interaction (pairing) (e.g. Beavers \& Wilson, 1970, Winant \& Browand, 1974) and the instabilities developing in these vortices (Sato, 1960), whereas in the fully developed far field no easily identifiable structures can be found any more in the turbulent shear layers (Rockwell \& Niccolls, 1972). Since the jet is constantly entraining fluid, an inward transverse velocity field is established outside the edges of the jet to replace the fluid masses that have been carried away and preserve continuity (e.g. Gutmark \& Wygnanski, 1976, Krothaplli et al., 1980).

The entrainment process is also closely related to the spreading of the jet (Namer \& Ötügen, 1988). The initial momentum is redistributed to account for the mobilization of the entrained fluid resulting in lower velocities of the flow masses imparting momentum. Thus the original mean mass flow emanating from the nozzle along with the entrained fluid need to be constantly redistributed downstream, through an 
outward mean transverse velocity component within the edges of the jet, to even larger cross sectional areas.

During the initial stages while the shear layer is thin the internal jet flow remains unaffected in the potential core region. There is evidence that at this stage the shear layer has in fact a confining effect on the internal flow (Grinstein et al., 1995). The mean streamwise velocity remains constant in the core region, or can even increase in a similar way that the velocity increases in the potential core in the entrance section of a pipe, to preserve continuity, due to the development of the boundary layers and the consequent mass flux reduction close to the boundaries. In a jet this effect is competing with the tendency of the jet to spread and increase its effective cross section. If the spreading is not sufficiently quick the velocity may increase downstream in the potential core. Thereafter, once the long side shear layers meet at the centre of the jet, the spreading becomes dominant and the centreline velocity is decreasing downstream (Namer \& Ötügen, 1988). In the case of the jet with no sidewalls (NENS, WENS), the development of the shear layers on the short sides forces the internal fluid towards the central part of the jet. Again the jet spreading assisted by the outward increased lateral velocity component is the competing mechanism maintaining lower velocities at the central area. It is interesting that these effects have not a clear and monotonic impact on the decay and spreading characteristics.

The development of a jet is dictated by the interplay of the above described mechanisms which are closely related. Key parameters in this interaction are the geometry of the nozzle (including its dimensions and aspect ratio) (Gutmark et al., 1999), and the dynamic characteristics of the jet (including jet momentum and Reynolds number (e.g. Namer \& Ötügen, 1988, Everitt \& Robins, 1978), as well as the initial boundary layers' characteristics and the initial turbulence levels) (Hussain et al., 1977). Comparing the jets with sidewalls (NEWS and WEWS) with the corresponding free jets (NENS and WENS); the investigation is focused on the effect of the boundary layers developing on the sidewalls in the first case in contrast to the short side shear layers of the latter case. 
Regarding the mean flow field the most characteristic differences due to the presence of the sidewalls (cases NEWS, WEWS) are the larger streamwise velocities established in the outer field (especially just after the potential region), along with the systematically smaller transverse velocities at all stations (Figure 5.1 and Figure 5.4). The combination of these characteristics is rather surprising since the larger outward transverse velocities of the jet without sidewalls would be expected to transfer momentum to the outer field more effectively assisted by the larger turbulent diffusion indicated by the turbulence terms $\left(u^{\prime}, v^{\prime},\langle u v\rangle\right)$. The only mechanism capable to produce the observed differences, counter fighting those mentioned above is the convection of longitudinal momentum by $2 \mathrm{D}$ roller structures established more stably in the cases of the jets with sidewalls (NEWS, WEWS). These rollers seem to constantly carry fluid from the inner field to the outer and vice versa, pumping momentum from the inner jet to the outer field, whereas the net outward flow is maintained lower than that of the free jet. In this latter case of the jets without sidewalls (NENS, WENS) the shear layers developing on the sort side edges of the jet are responsible for the destruction of the $2 \mathrm{D}$ rollers, increasing turbulence levels and decreasing the efficiency of momentum exchange by convection. The concept described above finds support in the work of Davies et al., (1975) and Moum et al., (1983) who have concluded that organized motions that exist in the fully developed plane turbulent jet play an important role on the spread of the flow and transport processes.

Based on the work of Grinstein, (2001), 3D ring like vortices can be suspected to develop at the edges of a rectangular jet. The presence of such structures is also evidenced by the undulations of the streamwise velocity profiles on the $x$ zplane at $x / D=6,25$ (Figure 5.3). The sidewalls seem to alleviate but not completely suppress these characteristics, indicating that even in the presence of sidewalls the flow field is not two dimensional. According to Marsters, (1980) the saddle back shape, observed in the spanwise distributions, may be due to the pressure field associated with curved streamlines, which would have a peak value along the centreline ( $x z$ plane) in the jet flow. This centrally located high-pressure region would drive secondary flows that could move high velocity fluid from the central part to the outer ends of the flow field. Tsuchiya et al., (1986) postulated that the saddle back profile is probably due to the formation of a retarded mixing region. In contrast to previous investigations the 
velocity undulations in the present work incorporate a lower peak at the central area of the profile.

Besides the presence of ring like structures a plausible mechanism explaining these attributes could be the instability of the jet front. The jet impulse is not sufficient to maintain a uniform velocity along the jet length. In an infinite jet the instability length scale would be dictated by the jet and fluid characteristics, and the instability effects would have been smeared out in the mean field. In rectangular jets either bounded by sidewalls or not, the length of the jet's long side imposes an additional length scale. Besides the shear layers developing at the sort sides of the jet, either as a boundary layer on the sidewall or as a free shear layer entraining fluid in the unbounded case, provide specific locations for the stabilization of instability undulations. The presence of a secondary peak in the present results for higher aspect ratio jet in comparison to the clear saddle back profiles of the previous lower aspect ratio investigations is compatible with this concept.

Regarding the spreading, the results presented in Figure 5.6 for $\operatorname{Re}=20,000$ indicate very similar behaviour for the four test cases in the first stages of development. Given that in the far field the roller structures have been destroyed even for the case of a jets with sidewalls (NEWS, WEWS), this effect may be attributed to the accumulated result of the higher outward transverse velocities, which eventually spread longitudinal momentum in a larger transversal area in the jet with no sidewalls (Deo et al., 2007c).

The velocity decay presents a different behaviour at $R e=20,000$ (Figure 5.9). Differences in the decay rate between the test cases are scarcely discernible just after the potential core where the centreline velocity of the jets with no sidewalls (NENS, WENS) almost collapse and take higher values, probably due to the confining action of the shear layers on the short sides of the jet, which lead to the relative increase of the longitudinal velocity in the central part of the jet as already discussed. It is interesting to note that the interplay of the mechanisms outlined in the previous, lead to different trends in relation to the Reynolds number. 
Both components of the rms velocity (Figure 5.11 and Figure 5.16) present higher values in the shear layers for the case of the jets with no sidewalls in the early stages of development. This trends may be associated with the more intensive outward transverse velocity observed in these areas although they are of a more limited and progressive nature. Close to the centreline, values may also indicate a small increase of the potential core in the case of the jets with sidewalls, since both rms components increase (i.e. penetrate) at a slower pace in this area.

Adopting the dependence of the transverse pressure distribution to the lateral velocity rms component one, predicted by Tennekes \& Lumley, (1972) based on order of magnitude analysis in developed flows, a plateau of lower pressure is established in the area within the shear layers after the potential region. Initially values are lower for the jets with no sidewalls but this relation is alleviated or even inversed downstream.

The triple velocity products represent turbulent transport of the Reynolds normal stresses by the turbulent velocities (Figure 5.24, Figure 5.26, Figure 5.28, and Figure 5.30) whereas the corresponding correlation is the skewness factor (Figure 5.32 and Figure 5.36). The monotonic increase of the skewness factor across the shear layers from negative values to positive and especially the formation of sharp peaks at the outer edges of the jet are illustrative of the presence of well defined roller structures in the case of the jets with sidewalls (NEWS, WEWS). On the contrary in the free jets (NENS, WENS) cases the early destruction of the 2D rollers and the increase of turbulence, smooth out the sharp peaks. 


\section{Chapter 6}

\section{CONCLUSIONS}

A systematic study has been performed of the influence of endplate, sidewalls and endplate and sidewalls on the development of turbulent rectangular jets of Reynolds number, $R e=10,000,20,000$ and 30,000 issuing from a smooth contraction nozzle of aspect ratio, $A R=15$. For each condition, the statistical properties of rectangular jet, arising from changes in the initial and boundary conditions were quantified.

The present study on the influence of endplate on free rectangular jets (without sidewalls, cases NENS and WENS), over an axial distance of $35 \mathrm{D}$ in the lateral direction revealed no significant effects of the presence or the absence of the endplate. It is almost impossible to identify differences larger than the uncertainty and of a systematic nature between the results of the two configurations with no sidewalls. It can almost be stated that the results of both NENS and WENS jets collapse in all the presented distributions.

The effect of sidewalls on the velocity field of turbulent rectangular air jet from a nozzle of aspect ratio 15 and of Reynolds number, $R e=10,000,20,000$ and 30,000 was investigated using hot wire anemometry over an axial distance 35 nozzle width. In this study, the two configurations NEWS and WEWS indicate that sidewalls have a clear effect on the development of the mean and turbulent flow field, which is different in the presence or absence of an endplate in the regions investigated. In the presence of the sidewalls, a systematic increase of the mean streamwise velocity values at the jet's edges was observed. The presence or the absence of the endplate seems not to have an effect on these trends. These trends have been evidenced in previous investigations (e.g. Chua \& Lua, 1998, Davies et al., 1975, Moum et al., 1983). These trends may be attributed to difference in the development of the structures (vortices) formed in the presence or absence of the sidewalls. In the presence of the sidewalls, these 2-D rollers seem to constantly carry the fluid from the inner field to the outer field, pumping the momentum from the inner jet to the outer jet. The presence of sidewalls is characterized by lower outward velocities where the presence of the endplates (WEWS) seems to have an effect resulting in larger values 
than the one without endplate (NEWS) in some locations. The presence of sidewalls and endplates has an effect on the development of the negative outward velocities formed at the edges of the jet. The presence of sidewalls is associated with higher negative velocities in these areas. Again the presence of the endplate does play a role (WEWS) leading to lower negative velocities in comparison to the case with no endplate (NEWS).

The presence of sidewalls has a significant effect on the jet spreading rates. We have found that the jets without sidewalls spread in nearly similar rates (NENS 0.126, WENS 0.129) but higher than when the sidewalls were implemented (NEWS 0.105, WEWS 0.112). The smaller outward velocities observed in the presence of the sidewalls are probably responsible for the slower spread rates in cases NEWS and WEWS. While the larger outward velocities found in the absence of the sidewalls as well as the higher rate of 3-D effects seem to spread the longitudinal momentum to a larger transverse area.

The presence of the sidewalls and endplates has a clear effect on the mass flow rate of the studied jets leading to higher values in the region $x / D=5-25$ compared to the jets without sidewalls. The differences between the configurations incorporating sidewalls and those which do not may be attributed to the two dimensional characteristics imposed by the sidewalls. In the early stages the mass flow at the central $x y$ plane is increasing slower in the absence of sidewalls due to the spreading in the spanwise direction. When the 3D jets with no sidewalls are beginning to attain an axisymmetric structure, the flow rate due to the rearrangement of the flow is increasing faster than for the two dimensional jets with sidewalls, which gradually lose their potential to entrain ambient fluid. The presence of the sidewalls was found to result in higher initial momentum probably due to the confinement of the jet, preventing the expansion of the jet and the diffusion of momentum in the spanwise direction (i.e. long dimension).

The influence of Reynolds number on the decay rate for each configuration was assessed. The decay rates of (NENS and WENS) where found nearly the same at all tested Reynolds numbers. The decay rates for these cases (NENS, WENS) were increased from $R e=10,000$ to $R e=20,000$ and then showed a small reduction from $R e=20,000$ to $R e=30,000$ for both configurations. This indicates that the decay rates 
of the tested jets in the absence of the sidewalls attain their asymptotic values at $R e=20,000$. But in the presence of the sidewalls (with or without endplate i.e. NEWS and WEW) the decay rates trends were found to be different when Reynolds number was varied. The presence of endplate and sidewalls (WEWS) result in the highest decay rates at all Reynolds number tested compared to the case without endplate endplates (NEWS). The increase of the decay rates of both jets with sidewalls (NEWS, WEWS) seems not have the saturation even at the highest Reynolds number i.e. $\operatorname{Re}=30,000$.

The presence of the sidewalls and endplates was found to strongly affect the turbulent field of the rectangular jet at $R e=20,000$. In the near field, it was found that the presence of the sidewalls lead to smaller turbulent velocities in the axial and lateral direction at the shear layers as well as towards the edges of the jet. But in the far field, a systematic increase of the rms velocities for cases incorporating sidewalls was observed with a higher rate in the presence of the endplate.

These observations are probably attributed to presence of more organized structures in the presence of the sidewalls preventing them to attain self-preserving behavior compared to the free jets (NENS, WENS) where profiles attained its self-similar behavior at about $x / D \geq 15$.

The Reynolds shear stresses $(u v)$ as well as third moment (i.e. $u^{3}, v^{3}, u^{2} v, u v^{2}$ ) lateral profiles show similar trends to that on the corresponding fluctuating velocities' moment, indicating the significant effects of the endplate and sidewalls on the development of the studied jets.

In the presence of the sidewalls, a monotonic increase of the skewness factor across the shear layers from negative values to positive and especially the formation of sharp peaks at the outer edges of the jet are illustrative of the presence of well defined roller structures.

Our investigation support the previously established understanding that these jets are strongly influenced by the various initial and boundary conditions. 


\section{Bibliography}

Abdel-Rahman A. A., Chakroun W. and Al-Fahed S. F.: 1997, LDA measurements in the turbulent round jet, Mech. Res. Comm. 24(3), 277-288.

Abramovich G. N.: 1963, The theory of turbulent jets. Massachusetts Institute of Technology. Cambrige, United States of America.

Antonia R. A., Browne L. W. B., Chambers A. J. and Rajagopalan, S.: 1983a, Budget of temperature variance in a turbulent plane jet, Int. J. Heat Mass Transfer 26, 41-48.

Antonia R. A., Browne W. B., Rajagopalan S. and Chambers, A. J.: 1983b, On organized motion of a turbulent planar jet, J. Fluids Mech. 134, 49-66.

AN-1003 user manual (Hot wire and film anemometry system) from A.A. Lab System LTD.

Ball C. G. and Pollard A.: 2007, A Review of Experimental and Computational Studies of Flow from the Round Jet, Internal Report CEFDL/2007/01, Queens University, Canada.

Beavers G. S. and Wilson T. A.: 1970, Vortex growth in jets, J. Fluid Mech. 44(1), 97-112.

Bernard P. S. and Wallace J. M.: 2002, Turbulent flow, John Wiley and Sons, New Jersey.

Bradbury L. J. S.: 1965, The structure of self-preserving turbulent planar jet, J. Fluid Mech. 23, 31-64.

Browne L. W. B., Antonia R. A., Rajagopalan S. and Chambers A. J.: 1982, Structure of complex turbulent shear flows, IUTAM Symposium, Marseille, pp. 411-419. 
Browne L. W. B., Antonia R. A. and Chambers A. J.: 1984, The interaction region of a turbulent planar jet, J. Fluid Mech. 149, 355-373.

Cavo, A., Lemonis G., Panidis, Th. and Papailiou, D.D.: 2007, Performance of a 12sensor vorticity probe in the near field of a rectangular turbulent jet, Exp. Fluids 43, pp. 17-30.

Çengel Y. A.: 1997, Introduction to thermodynamic and heat transfer, The McGrawHill Companies, Inc. United States of America.

Chua L.P. and Lua A.C.: 1998, Measurements of a confined jet, Phys. Fluids 10 (12)

Chambers A. J., Antonia R. A. and Browne L. W. B.: 1985, Effect of symmetry and asymmetry of turbulent structures on the interaction region of a plane jet, Exp. Fluids 3, 343-348.

Crow S. C. and Champagne F. H.: 1971, Orderly structure in jet turbulence, J. Fluid. Mech. 48(3), 547-591.

Daves A. E., Keffer J. F. and Baines W. D.: 1975, Spread of heated plane turbulent jet, Phys. Fluids 18 (7), pp. 770-775

Deo R. C.: 2005, Experimental investigations of the influence of Reynolds number and boundary conditions on a plane air jet, Ph.D. Thesis, School of Mechanical Engineering, University of Adelaide, Australia.

Deo R. C., Nathan G. J. and. Mi J.: 2007a, The influence of nozzle aspect ratio on a plane jet. Exp. Therm. Fluid Sci. 31 pp 825-838.

Deo R. C., Nathan G. J. and. Mi J.: 2007b, The influence of nozzle exit geometric profile on a statistical properties of a turbulent plane jet. Exp. Therm. Fluid Sci. 32 pp 545-559. 
Deo R. C., Nathan G. J. and Mi J: 2007c, Comparison of turbulent jets issuing from rectangular nozzle with and without sidewalls. Exp. Therm. Fluid Sci. 32 (2) pp 596606.

Deo R. C., Mi J., and Nathan G. J.: 2008, The influence of Reynolds number on a plane jet. Phys. Fluids (20), 075108.

Douglas J. F., Gasiorek J. M. and Swaffield J. A.: 1995, Fluid Mechanics, $3^{\text {rd }}$ edition, Longman Scientific \& Technical. Printed in Singapore.

Everitt K. W. and Robins A. G.: 1973, The development and structure of turbulent plane jets, J. Fluid. Mech. 88, 563-583.

Frank M. W.: 1994, Fluid Mechanics, International edition, $3^{\text {rd }}$ edition, McGraw-Hill companies, Inc. Printed in the United States of America.

Goldstein R. J.: 1996, Fluid Mechanics Measurements, second edition, Taylor \& Francis, Printed in the United States of America.

Grinstein F.F.: 2001, Vortex dynamics and entrainment in rectangular free jets, J. Fluid Mech. 437, pp. 69-101

Grinstein F. F., Glauser M. N. and George W. K.: 1995, Vorticity in Jets, S.I.Green Ed., Kluwer Academic Publisher, Netherland, chapter III, Fluid Vorticity, pp. 65-94.

Gutmark E. and Wygnanski I.: 1976, The planar turbulent jet, J. Fluid Mech. 73(3), $465-495$.

Gutmark E.J. and Grinstien F.F.: 1999, Flow control with non circular jets, Annu. Rev. Fluid Mech. 31:239-272.

Heskestad G.: 1965, Hot-wire measurements in a plane turbulent jet, Trans. ASME, J. Appl. Mech. 32(721-734). 
Hitchman G. J., Strong A. B., Slawson P. R. and Ray G.: 1990, Turbulent planar jet with and without confining walls, AIAA J. 28(10).

Hussain A. K. M. F. and Clark A. R.: 1977, Upstream influence on the near field of a planar turbulent jet, Phys. Fluids (20) 9, 1416-1426.

Kotsovinos N.: 1978, A note on the conservation of axial momentum of a turbulent jet, J. Fluid Mech. 87(Part 1), 55-63.

Krothapalli A., Baganoff D. and Karamcheti K.: 1981, On the mixing of rectangular jet, J. Fluid Mech. 107, 201-220.

Lazanova M. and Stankov P.: 1998, Experimental investigation on the similarity of a 3D rectangular turbulent jet, Exp. Fluids 24, 470-478.

Lemieux G. P. and Oosthuizen P. H.: 1985, Experimental study of behaviour of planar turbulent jets at low Reynolds numbers, AIAA J. pp. 1845-1846.

Moum J. N, Kawall J. G. and Keffer J. F.: 1983, Coherent structures within the plane turbulent jet, Phys. Fluids 26 (10), pp. 2939-2945

Marsters G. F. and Fotheringham J.: 1980, The influence of aspect ratio on incompressible turbulent flows from rectangular slots, Aeronaut. Quart XXXI (4), 285-305.

Mi J., Nathan G. J. and Luxton R. E.: 2000, Centerline mixing characteristics of jets from nine differently shaped nozzles, Exp. Fluids 28, 93-94.

Mi J., Nathan G. J. and Nobes D. S.: 2001, Mixing characteristics of axisymmetric free jets from a contoured nozzle, an orifice plate and a pipe, J. Fluid. Eng. 123, 878883.

Mi J., Deo R. C. and Nathan G. J.: 2005, Characterization of turbulent jets from highaspect-ratio rectangular nozzles, Phys. Fluids 17(6). 
Namer I. and Ötügen M. V.: 1988, Velocity measurements in a planar turbulent air jet at moderate Reynolds numbers, Exp. Fluids 6, 387-399.

Norman B.: 1967 Hot-wire anemometer calibration at high subsonic speeds, DISA Information 5 .

Papadakis M. and Staiano M. W.: 1993, Experimental and computational investigation of free and impinging planar jets, AIAA Paper 93-0654, 1-11.

Pope S. B.: 2002, Turbulent Flows, Cambridge University Press, UK.

Quinn W. R.: 1992, Turbulent free jet flows issuing from sharp-edged rectangular slots: The influence of slot aspect ratio, Experimental Thermal and Fluid Science 5, 203-215.

Rajaratanam 1976, Turbulent jets, Elsevier scientific publishing company.

Romeos A., Lemonis G., Panidis Th., and Papailiou D.: 2007, Multisensor hot wire vorticity probe measurements of the formation field of two corotating vortices, Flow, Turbulence and Combustion (2009) 83:153-183.

Rockwell D. O. and Nicholas, W. O.: 1973, Natural breakdown of planar jets, J. Basic. Eng 94, 720-730.

Sato, H.: 1960, The stability and transition of a two-dimensional jet, J. Fluid Mech.7, 53-80.

Schneider W: 1985, Decay of momentum flux in submerged jets. J. Fluid Mech. 54, 91-110.

Seo W. I., Kwon S. J.: 2005, Experimental investigation of Three-dimensional nonbuoyant rectangular jets, Journal of Engineering Mechanics. July 2005, 733-746. 
Sfeir A.: 1979, Investigation of three dimensional turbulent rectangular jets, AIAA J. 17(10), 1055-1060.

Sforza P. M., Steiger M. H. and Trentacoste N.: 1966, Studies on three-dimensional viscous jet, AIAA J. 4(5), 800-806.

Suresh P. R., Srinivasan K., Sundararajan T., Das K.: 2008, Reynolds number dependant of a plane jet development in the transition regime, Phy. Fluids 20, 044105.

Tennekes H., Lumley J. L.: 1972, A first course in turbulence. MIT Press.

Trendscoste, N. and Sforza, P.: 1967, Further experimental results for threedimensional re jets, AIAA J. 5(5), 885-890.

Tsuchiya Y., Horikoshi C. and Sato T.: 1985, On the spread of rectangular jets, Exp. Fluids 4, 197-204.

Winant C. D. and Browand F. K.: 1974, Vortex pairing: the mechanism of turbulent mixing-layer growth at moderate Reynolds number, J. Fluid Mech., 63:2:237-255. 